Prepared for the U.S. Department of Energy

under Contract DE-AC05-76RL01830

\title{
An Adaptive Landscape Classification Procedure Using Geoinformatics and Artificial Neural Networks
}

AM Coleman

June 2008

\section{Pacific Northwest}

NATIONAL LABORATORY

Proudly Operated by Battelle Since 1965 


\title{
DISCLAIMER
}

This report was prepared as an account of work sponsored by an agency of the United States Government. Neither the United States Government nor any agency thereof, nor Battelle Memorial Institute, nor any of their employees, makes any warranty, express or implied, or assumes any legal liability or responsibility for the accuracy, completeness, or usefulness of any information, apparatus, product, or process disclosed, or represents that its use would not infringe privately owned rights. Reference herein to any specific commercial product, process, or service by trade name, trademark, manufacturer, or otherwise does not necessarily constitute or imply its endorsement, recommendation, or favoring by the United States Government or any agency thereof, or Battelle Memorial Institute. The views and opinions of authors expressed herein do not necessarily state or reflect those of the United States Government or any agency thereof.

\author{
PACIFIC NORTHWEST NATIONAL LABORATORY \\ operated by \\ BATTELLE \\ for the \\ UNITED STATES DEPARTMENT OF ENERGY \\ under Contract DE-AC05-76RL01830
}

Printed in the United States of America

Available to DOE and DOE contractors from the

Office of Scientific and Technical Information,

P.O. Box 62, Oak Ridge, TN 37831-0062;

ph: (865) 576-8401

fax: $(865)$ 576-5728

email: reports@adonis.osti.gov

\footnotetext{
Available to the public from the National Technical Information Service, U.S. Department of Commerce, 5285 Port Royal Rd., Springfield, VA 22161 ph: (800) 553-6847 fax: $(703) 605-6900$

email: orders@ntis.fedworld.gov

online ordering: http://www.ntis.gov/ordering.htm
}

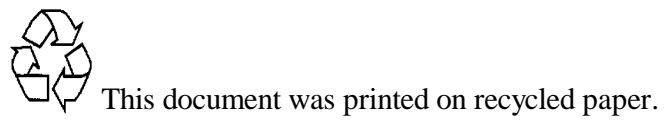




\section{An Adaptive Landscape Classification Procedure Using Geoinformatics and Artificial Neural Networks}

\section{DISSERTATION}

André Michael Coleman

Submitted in part fulfillment of the requirements for the degree of Master of Science in Geographical Information Systems

Faculty of Earth and Life Sciences

Vrije Universiteit, Amsterdam

The Netherlands

June, 2008 


\section{Abstract}

The Adaptive Landscape Classification Procedure (ALCP), which links the advanced geospatial analysis capabilities of Geographic Information Systems (GISs) and Artificial Neural Networks (ANNs) and particularly Self-Organizing Maps (SOMs), is proposed as a method for establishing and reducing complex data relationships. Its adaptive and evolutionary capability is evaluated for situations where varying types of data can be combined to address different prediction and/or management needs such as hydrologic response, water quality, aquatic habitat, groundwater recharge, land use, instrumentation placement, and forecast scenarios. The research presented here documents and presents favorable results of a procedure that aims to be a powerful and flexible spatial data classifier that fuses the strengths of geoinformatics and the intelligence of SOMs to provide data patterns and spatial information for environmental managers and researchers.

This research shows how evaluation and analysis of spatial and/or temporal patterns in the landscape can provide insight into complex ecological, hydrological, climatic, and other natural and anthropogenic-influenced processes. Certainly, environmental management and research within heterogeneous watersheds provide challenges for consistent evaluation and understanding of system functions. For instance, watersheds over a range of scales are likely to exhibit varying levels of diversity in their characteristics of climate, hydrology, physiography, ecology, and anthropogenic influence. Furthermore, it has become evident that understanding and analyzing these diverse systems can be difficult not only because of varying natural characteristics, but also because of the availability, quality, and variability of spatial and temporal data. Developments in geospatial technologies, however, are providing a wide range of relevant data, and in many cases, at a high temporal and spatial resolution. Such data resources can take the form of high-dimensional data arrays, which can difficult to fully use. Establishing relationships among high-dimensional datasets through neurocomputing based patterning methods can help 1) resolve large volumes of data into a meaningful form; 2) provide an approach for inferring landscape processes in areas that have limited data available but that exhibit similar landscape characteristics; and 3) discover the value of individual variables or groups of variables that contribute to specific processes in the landscape. 


\section{Table of Contents}

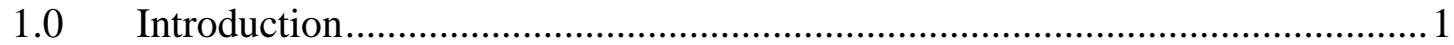

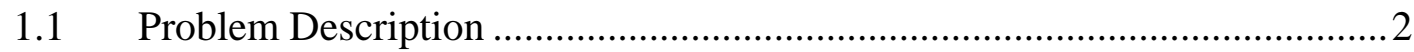

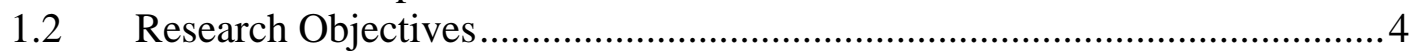

1.3 The Adaptive Landscape Classification Procedure ...................................... 4

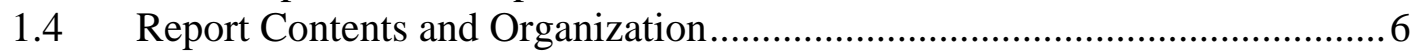

2.0 Landscape Classification and Modeling .................................................... 8

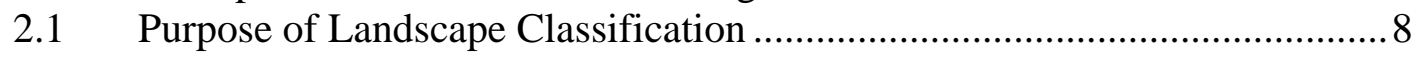

2.2 Current Methods of Landscape Classification............................................. 11

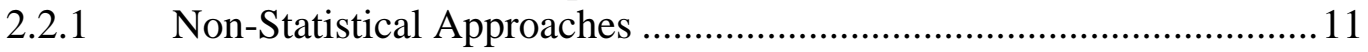

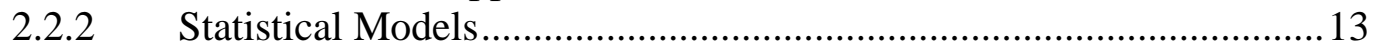

2.2.3 Maximum Likelihood Classifier .......................................................... 15

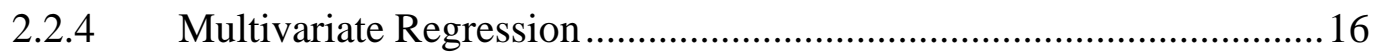

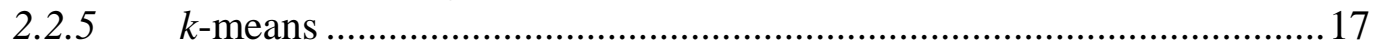

2.2.6 Ward's Hierarchical Clustering ........................................................220

2.2.7 Artificial Neural Networks ............................................................22

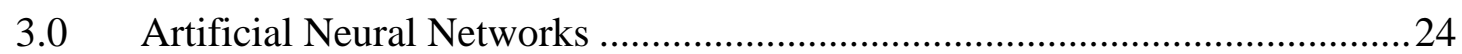

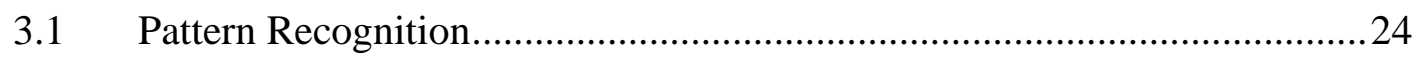

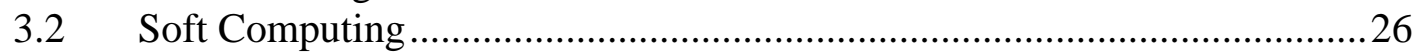

3.3 Fundamental Basis of Artificial Neural Networks...................................26

3.4 Common Types of Artificial Neural Networks ........................................32

3.4.1 Supervised Artificial Neural Networks............................................... 32

3.4.2 Unsupervised Artificial Neural Networks .............................................. 35

4.0 The Adaptive Landscape Classification Procedure ......................................46

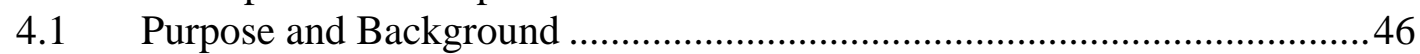

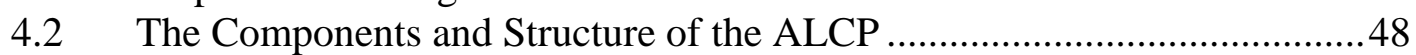

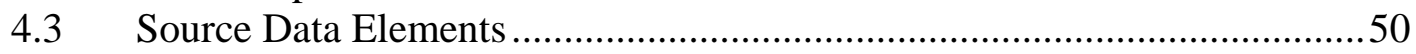

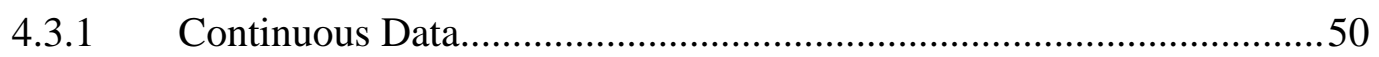

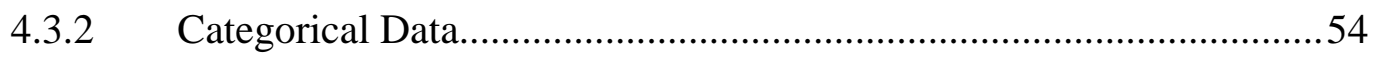

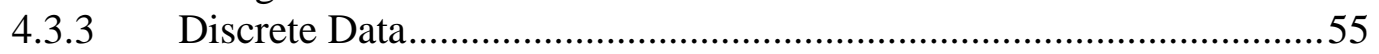

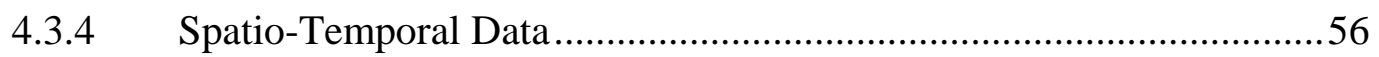

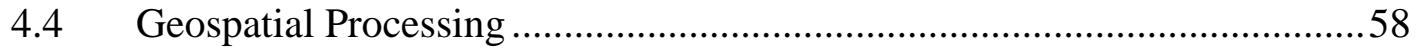

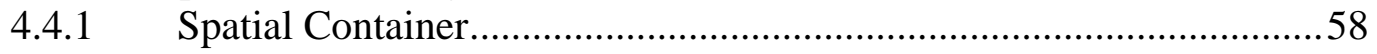

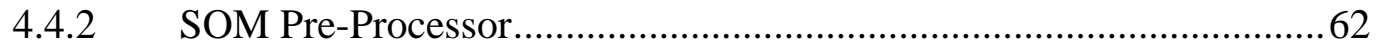

4.5 SOM Model and Post-Processor................................................................63

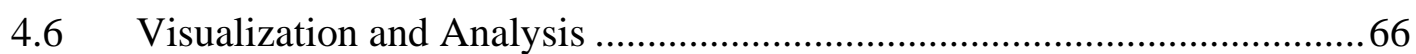

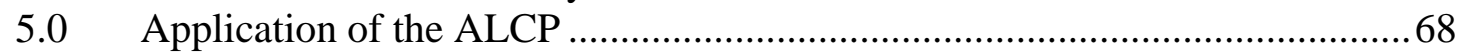

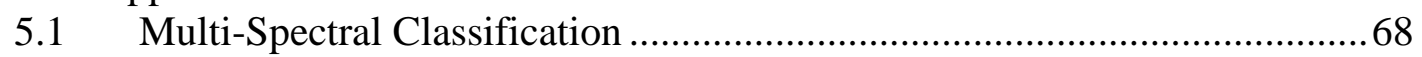

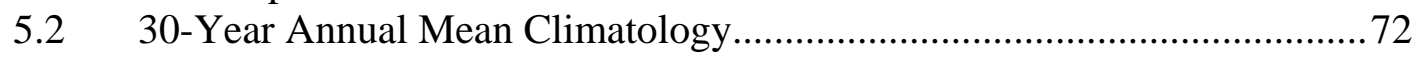

5.3 Hydrologic Properties and Landscape Characteristics ............................... 78

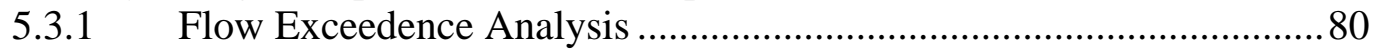

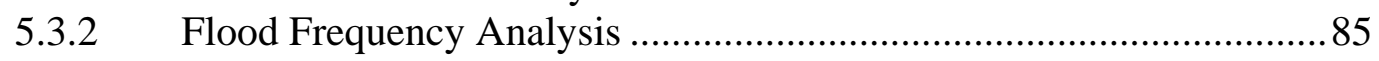

5.3.3 Landscape Characteristics Analysis to Determine Hydrologic

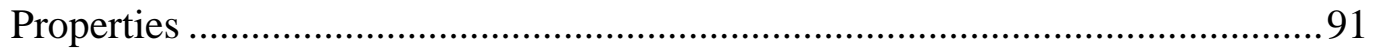

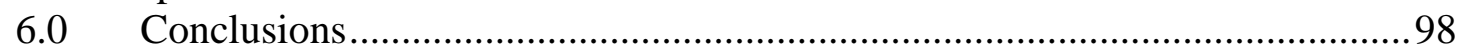

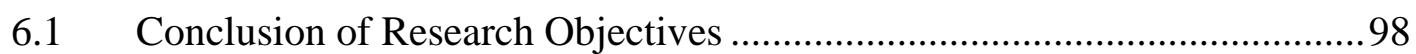

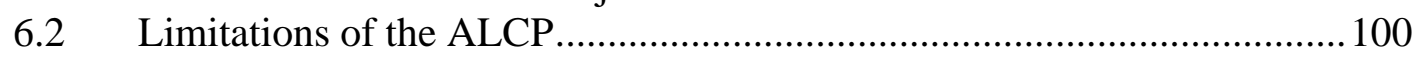

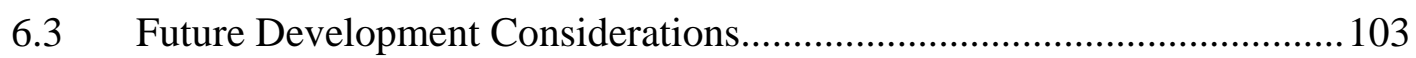

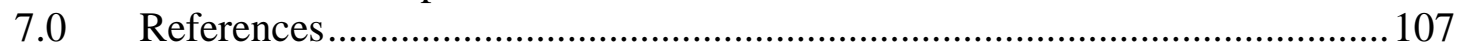




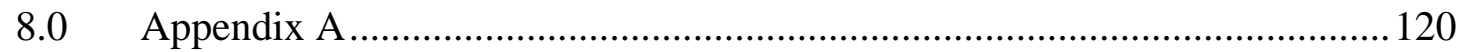

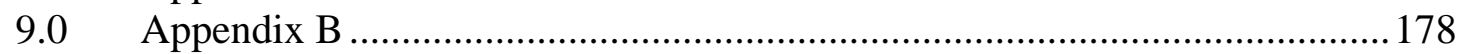




\section{List of Figures}

Figure 2.1. The basic process of classification groups univariate or multivariate input data similar or near-similar data into groupings based on a set of rules.

Figure 2.2. An example of an observationally interpreted landscape classification using variables of geology, physiography, vegetation, climate, soils, land use, wildlife distributions, and hydrology (Thorson et al., 2003).

Figure 2.3. Hierarchical approach to landscape classification where elements operating at broad temporal and spatial scale have more dominance over system processes (adapted from Snelder and Biggs 2002).

Figure 2.4. A sample of 8000 points in the initial phase of k-means processing with random starting "seeds" placed in the input data space (a) and the final convergence stage (b). The cluster center, $c_{j}$, is indicated by the large colored dot and parameter $\mathrm{k}=5$ resulting in five distinctive cluster areas (Pelleg, 2004).

Figure 2.5. The ISOCluster model incorporates $k$-means, ISODATA, and the Maximum Likelihood Classifier, to organize and classify multivariate data.

Figure 2.6. An example of the agglomerative hierarchical clustering for dominance of tree species in Wisconsin, USA (Bolliger et al., 2004).

Figure 3.1. This illustration exemplifies an anomaly (far right) in a regular pattern space. The mind immediately picks up on the abnormality, which thus becomes a point of interest (SFCC, 2007).

Figure 3.2. This set of objects relates the brains' natural ability to recognize patterns and fill in missing information. Note the only objects that actually exist in this illustration are four incomplete circles with varying amounts of missing information. Through pattern-recognition, four complete circles and a square are comprehensible (SFCC, 2007).

Figure 3.3. This illustration exemplifies the concept of proximity where the pattern on the left is viewed as a series of separate objects, the one in the middle is viewed as a single object (although it consists of separate objects), and the one on the right is viewed holistically as a single complex object composed of similar objects with different orientations (SFCC, 2007).

Figure 3.4. A simplified graphic representation of a neuron cell processing and transmitting information from cell to cell (adapted from Lingireddy and Brion, 2005).

Figure 3.5. A common and simple ANN schematic that represents the flow of information from the input data, to the receipt of the input neurons, to weighting and 
evaluating of data, to signal adjustments in the hidden layer, and finally the resulting output data (adapted from Principe et al., 2000).

Figure 3.6. Result of a simple multi-layer perceptron ANN evaluating the MSE over model iterations. Once a sufficient MSE is reached, the optimal solution is obtained, meaning ANN output values closely match the training values and an underlying relationship has been established between input data vectors and resulting output. 31

Figure 3.7. A representation of the single-layer Self-Organizing Map process as it presents data to the network, competes, and maps organized data clusters to a defined 1-D, 2-D, or 3-D topology.

Figure 3.8. A representation of the random weight initialization that occurs in the first phase of the SOM learning process. Input codebook vectors are presented to the randomly weighted neurons and the organization and learning process begins.

Figure 3.9. A Gaussian function is often applied to a time-decaying kernel neighborhood to update the "winning" neuron and those in the effective area. This process makes the SOM learning efficient and stable. The kernel is defined by the center point and the kernel neighborhood is represented by the red rings, which become smaller with each time-step.

Figure 3.10. The SOM process captured at multiple iterations reveals the competition, learning, and projection of neurons over the input data space. Note that with an increase in iterations, the decaying kernel neighborhood function has less influence on the overall network structure and focuses on the learning and competition with individual neurons and their immediate neighbors.

Figure 3.11. A randomly generated $450 \times 300$ RGB image with 135,000 values used as input to a SOM training process.

Figure 3.12. The random initialization of neuron weights in a 2-D grid is presented. Each pixel in the grid is representative of a single neuron.

Figure 3.13. The final result of the SOM training from a $450 \times 300$ dataset reduced and organized into a $64 \times 64$ grid.

Figure 3.14. A $32 \times 32$ 2-D trained SOM using the randomly generated source data presented in Figure 3.11 .

Figure 4.1. Four variables for 10 sub-basin areas are presented to illustrate different data patterns in the landscape.

Figure 4.2. An overview of the structure and flow of the ALCP.

Figure 4.3. Two examples of continuous datasets that are raster-based and characterized by smooth transitions between the attributes. 
Figure 4.4. Categorical data comprise a dataset classified by pre-defined groupings as presented in the two examples.

Figure 4.5. Spatio-temporal data representing a 1-day maximum temperature forecast condition from the National Digital Forecast Database (NDFD) meteorology model (NWS, 2007).

Figure 4.6. A spatio-temporal dataset representing snow-water equivalent conditions for a given day. New data results are produced daily from the United States National Oceanic and Atmospheric Administration's Snow Data Assimilation Model (SNODAS) (NOHRSC, 2007).

Figure 4.7. The sub-watersheds presented in this figure serve as a spatial container for data harvesting and compilation of data exhibiting multiple-scales and data types.

Figure 4.8. The basic process flow and function of the spatial container (as represented by sub-watersheds) within the ALCP. The input neurons are representative of the vector codebook patterns containing the multiple datasets that are used in the classification.

Figure 5.1. Raster-based $3 \times 3$ kernel neighborhood, where $X$ represents the kernel pixel.

Figure 5.2. The upper four figures display the Landsat MSS input data space (represented by class-colored cubes) and the final projected SOM neurons (represented by class-colored spheres). The bottom two figures display the final projected SOM in structured space.

Figure 5.3. An overview of the 3075 ALCP spatial containers used for analysis on the North Fork of the Clearwater River watershed.

Figure 5.4. The input data space (colored cubes) and random-weighted neurons (spheres) for the North Fork Clearwater three-parameter climate data are represented in (a). The final projected SOM, a three-dimensional $2 \times 2 \times 2$ cubic topology network, is presented in (b).

Figure 5.5. The ALCP analysis/spatial classification of 30-year annual mean climate data in the North Fork of the Clearwater watershed. Existing meteorology stations are noted with the red triangles.

Figure 5.6. Linear regression analysis evaluating the relationship of elevation to precipitation, maximum temperature, and minimum temperature.

Figure 5.7. Box-and-whisker plots for precipitation minimum temperature and maximum temperature for each SOM-determined cluster.

Figure 5.8. Bar graph representing the total area occupied by each SOM-clustered class as represented in Figure 5.5. Note that existing meteorology collection stations exist in Class 2, Class 6, and Class 8. 
Figure 5.9. A total of 160 headwater catchments were derived for hydrologic and landscape analysis. The selected basins represent approximately $63 \%$ of the total watershed area.

Figure 5.10. Area distribution of the 160 sub-basins sampled for analysis. 80

Figure 5.11. Monthly values of $80 \%$ flow exceedence for all 160 test sub-basins. Flow units are in cubic feet per second (cfs).

Figure 5.12. Monthly values of $20 \%$ flow exceedence for all 160 test sub-basins. Flow units are in cubic feet per second (cfs)......

Figure 5.13. Final SOM projection for (a) Q80 and (b) Q20.

Figure 5.14. Mean values of each SOM cluster per month for (a) Q80 and (b) Q20.

Figure 5.15. Spatial mapping of the Q80 and Q20 SOM cluster results. The classes are sorted based on flow with Class 1 being the lowest and Class 8 the highest......85

Figure 5.16. Flood frequency values representing both flood magnitude and return period for each of the test basins.

Figure 5.17. Mean cluster values per return period for flood frequency analysis...... .88

Figure 5.18. SOM classification of flood frequency data for three return intervals over 160 sub-basins.

Figure 5.19. Linear regression plots testing the relationship of sub-basin area to flow magnitude for 2-, 10-, 100-, and 500-year return periods.

Figure 5.20. 100-year flood frequency regression plot with point members symbolized by their assigned SOM cluster.

Figure 5.21. Spatial mapping of the nine-period flood-frequency SOM cluster results. The classes are sorted based on mean flow values within each cluster, where Class 1 represents the lowest flows and Class 8 the highest.

Figure 5.22. The input data space (colored cubes) and final projected neurons (spheres) in (a) natural projection space and (b) structured representation. The final SOM structures represent the clustering of 10 landscape metrics. Note that one neuron, best viewed in (b), was not used, indicating a sufficient number of neurons used.

Figure 5.23. Bar graphs indicating (a) the overall similarity in the landscape and Q20 class boundaries, and (b) the degree of class change for those basins that were identified as being dissimilar. 
Figure 5.24. Similarity index map showing likeness and difference between two independent cluster analyses, using 1) spatial landscape metrics, and 2) Q20 regression equations and landscape metric data to feed the regression equations. .95

Figure 6.1. A design concept for a hybrid ANN model combining the unsupervised SOM classification with a supervised ANN such as the Multi-Layer Perceptron, resulting in a supervised classification of spatial data.

Figure 6.2. The concept of SOM Attribute Weighting is presented as a potential method for assigning data theme weights is the classification process. 105 


\section{List of Tables}

Table 3.1. Common activation functions used in ANN models (StatSoft, 2003) ......30

Table 3.2. A small sample of Fisher's multivariate Iris flower dataset (Fisher, 1936).

Table 4.1. Terrain-based data processed and extracted within the ALCP.

Table 5.1. Classification number and descriptions of the Landsat MSS dataset.

69

Table 5.2. Class-assigned pixel counts for each SOM neuron are presented. The dominant count value, marked in bold-italic typeface, is declared the "class winner" for the neuron.

Table 5.3. A confusion matrix showing classified and misclassified data by class. Values indicated by bold-italic typeface indicate correctly classified values. All data are presented as percentages.

Table 5.4. An example demonstrating the dominant class and degree of class difference for data assigned to a given neuron.

Table 5.5. Source spatial data the USGS used to support and develop multivariate regression equations (left), and the data source used in the landscape analysis test (right). 


\section{Disclaimer}

The results presented in this thesis are based on my own research at the Faculty of Earth and Life Sciences of the Vrije Universiteit Amsterdam.

All assistance received from other individuals and organizations has been acknowledged and full reference is made to all published and unpublished sources.

This thesis has not been submitted previously for a degree at any institution.

Signed:

Date:
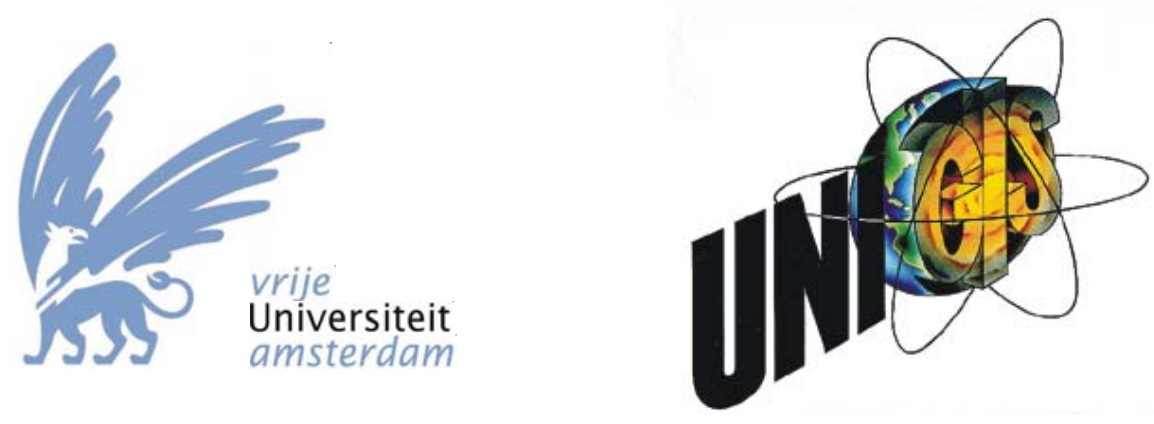


\section{Acknowledgements}

First and foremost, my gratitude and love are expressed to my wife, Laurie, and my children, Isabella and Sophia, for the countless hours I have spent fulfilling a desire to increase my knowledge in the sciences of geography, geographic information, and hydrology. My ultimate purpose in this effort is for no other reason than to help gain a better understanding of the beauties and complexities that lie in our natural world.

I would also like to express my appreciation for the love and support given to me by my greater family. Your words and actions throughout my studies have given me much needed strength.

None of this research would have been possible without my friend and mentor Lance W. Vail, whom repeatedly planted the seed sprouting the growth of this research. While it took some three years just to conceptualize what this seed was and its potential fruits, I'm grateful for the introduction to the crazy and amazing world of machine learning and neural networks.

My appreciation and gratitude are extended to my advisor, Professor Andrea Fabbri, whom graciously accepted the offer to supervise me through this process. Despite my trepidation after our first meeting (the questions you posed to me at this time left me thinking for two months!), I am indebted to you for your knowledge, thoughtprovoking insights, and prompting me to seek real meaning behind words commonly misused.

Lastly, I wish to acknowledge and thank the UNIGIS program director, Professor Henk Scholten, and the rest of the quality instructors and staff involved in the UNIGIS Amsterdam program. With this year being the $15^{\text {th }}$ anniversary of the program, this is a significant occasion and speaks very favorably to all the people who contribute to the success of the program. This has truly been an uplifting and rewarding experience providing a unique international perspective into the field of Geographic Information Sciences.

I am indebted to and humbled by all of you.

With my deepest regards,

André M. Coleman

May, 2008 


\section{Acronyms}

\begin{tabular}{|c|c|}
\hline $\mathrm{Al}$ & Artificial Intelligence \\
\hline ALCP & Adaptive Landscape Classification Procedure \\
\hline AML & Arc Macro Language \\
\hline ANN & Artificial Neural Network \\
\hline ART & Adaptive Resonance Theory (network) \\
\hline CVS & Concurrent Versions System \\
\hline $\begin{array}{l}\text { DEM } \\
\text { DNA }\end{array}$ & $\begin{array}{l}\text { Digital Elevation Model } \\
\text { deoxyribonucleic acid }\end{array}$ \\
\hline GIS & Geographic Information System \\
\hline GISc & Geographic Information Science \\
\hline GLCP & Global Land Cover Characterization Program \\
\hline JDEVS & Java Discrete Events System \\
\hline LVQ & Learning Vector Quantization \\
\hline MLC & Maximum Likelihood Classifier \\
\hline MLP & Multi-Layer Perceptron \\
\hline MSE & Mean Square Error \\
\hline MSS & Multi-Spectral Scanner \\
\hline NASA & National Aeronautics and Space Administration \\
\hline NDFD & National Digital Forecast Database \\
\hline NDVI & Normalized Difference Vegetation Index \\
\hline NED-H & National Elevation Dataset Hydrologic Derivatives \\
\hline NLCD & National Land Cover Database \\
\hline PCA & Principal Component Analysis \\
\hline pdf & Probability Distribution Function \\
\hline PNN & Probabilistic Neural Network \\
\hline PRISM & Parameter-elevation Regressions on Independent Slopes Model \\
\hline Q20 & 20\% Flow Exceedence \\
\hline Q80 & 80\% Flow Exceedence \\
\hline RBF & Radial Basis Function (network) \\
\hline RGB & Red Green Blue (color scheme) \\
\hline RNN & Recurrent Neural Network \\
\hline SAGA & System for an Automated Geographical Analysis \\
\hline SDM & Spatial Data Modeler \\
\hline SOM & Self-Organizing Map \\
\hline SRTM & Shuttle Radar Topography Mission \\
\hline SVM & Support Vector Machine \\
\hline USACE & United States Army Corps of Engineers \\
\hline USGS & United States Geological Survey \\
\hline
\end{tabular}




\subsection{Introduction}

Environmental management and research across a heterogeneous landscape provides challenges for consistent evaluation and understanding of natural processes. A heterogeneous landscape can exist in a wide variety of forms ranging from differences in specific hydrologic processes such as streamflow, groundwater recharge, rates of erosion or different ecological phenomena including biotic diversity, patch densities, and community dynamics. The magnitude of heterogeneity is variable and subject to the domain of study. For instance, an evaluation of stream temperatures for the purpose of understanding water quality and fish survival issues may present a limited domain of stream temperatures that are possible within the area of study. The classification of heterogeneous landscapes offers the ability to better understand individual or collective variables that contribute to natural processes and responses in the landscape.

The classification of landscapes over large spatial domains can present unique challenges due to the availability and diversity of data. With the exception of designated and protected research areas throughout the world, such as experimental watersheds and forests, detailed data collections are often limited to small areas with a specific research focus, largely due to the expense of carrying out large-scale research studies. While advances in automated data collection methods including space-based sensor platforms and field instrumentation have dramatically increased in their availability and reliability in the past three decades, there still remains a fundamental issue of retrieving sufficient information on the ground to develop a relationship between sensor and ground conditions; this step is vital to effectively make use of data across the entire landscape. For example, a meteorology instrument station collects weather information for one specific location in space and thus knowledge in between this station and others have a large degree of uncertainty. Similarly for space-based sensors, without an on-the-ground study, there is no way to relate spectral signatures to real elements in the landscape.

The research presented here identifies a procedure, showing positive results, that provides a powerful and adaptive procedure capable of processing large volumes of complex data, discovering relationships and patterns in the data, and reducing the data complexity to a more meaningful form by classifying common data patterns. The developed procedures can be used to propagate detailed information learned from a given spatial domain to other areas in the landscape without the same level of 
detailed information. This notion, among other things, allows for the intelligent and efficient pre-planning of research and monitoring studies to effectively capture the unique aspects in the landscape, and then apply the learned information to the "data gaps" or areas in between the specific study sites. The procedure is well suited for use in adaptive environmental modeling, research, monitoring, and management, as well as predictive and solution capabilities for a wide range of topic areas (i.e., determine probable locations of groundwater recharge zones, ideal restoration and/or protection areas, field sampling and instrument location sites, land use assessment, "what-if" scenarios for various environmental impacts, etc.) and is specifically intended to be adaptive in the types of data that can be used and the problem sets it can be used for (i.e., not necessarily limited to addressing landscape-based questions).

\subsection{Problem Description}

The fundamental problem this research attempts to address is whether or not it is possible to have rich knowledge in a given domain of space and time in the landscape and convey this knowledge to other areas in the landscape that exhibit limited knowledge, yet possess some similar properties. The implications of finding an answer to this question can be significant in terms of understanding landscape processes at a finer scale, which enhances our ability to monitor and manage these landscapes. For example, the United States Geological Survey (USGS) currently maintains a nationwide network of approximately 8,900 gages to monitor streamflow. Each year, because of budget constraints, many of these gages are permanently taken out of service. The impact of using known and measured streamflow information along with other metrics defining the landscape, and propagating this information to other areas without stream gages could lead to efficient use of available funds by prioritizing the value of a gage in terms of the uniqueness of the watershed it represents, and thus making informed decisions when removing gages. Additionally, the USGS currently relies on multivariate regression formulas built from $20+$ years of measured data to estimate streamflow characteristics in watersheds without instrument data. With anticipated changes in climate, particularly in mountain environments, regression formulas may be of less value since past data records may not be indicative of future conditions. The same concept as described for USGS stream gage data can be brought forth to assist in propagating knowledge across the 
landscape for various in situ data collection such as stream temperature, rates of erosion, groundwater recharge, and wildland fire potential.

Evaluation and analysis of spatial and temporal patterns in the landscape can provide knowledge and understanding of complex ecological and hydrological processes. Landscape patterns are not random, rather a structure underlies their variability. The patterns are driven and developed by a complex array of abiotic and biotic factors such as topography, climate, macroclimate, soils, ecosystem function, and anthropogenic influence (Turner et al., 2003). The spatial patterns of various elements in the landscape have a direct relationship with the processes in the landscape. The use of geoinformatics and Artificial Neural Networks (ANNs), particularly Self-Organizing Maps (SOMs), is proposed as a method to discover patterns in the landscape and system functions between areas in the landscape that are not only spatially disjointed, but dissimilar in their available data.

The use of ANNs is well-established in many sciences including genomics, risk analysis, forecasting, artificial intelligence, medicine/biomedicines, and more. Although a review of literature indicates both successes and failures using ANNs, the reviews of the successful applications show what is possible. As stated by Govindaraju and Rao (2000), "Researchers claim to be drawn to artificial neural networks because they possess desirable attributes of universal approximation, ability to learn from examples without the need for explicit physics, and the capability of processing large volumes of data at high speeds." The use of ANNs appears to be effective for understanding complex datasets and in the field of remote-sensing, these methods have proved themselves in the realm of research and are now emerging into commercial applications. From a review of the literature, it is clear the use of ANNs in Geographic Information Science (GISc) is still quite limited, even in the research domain.

The specific problems being addressed in this research are to:

- resolve and provide meaning to large amounts of spatial data that exist at different scales and come from different sources.

- explore the value of ANN models within the realm of GISc to discover similarities in complex data patterns and infer landscape processes in areas that have limited data available but exhibit similar landscape characteristics. 
- discover the value of individual variables or groups of variables that contribute to specific processes in the landscape.

\subsection{Research Objectives}

The goal of the study reported here is to research and integrate geospatial processing methodologies using ANNs, particularly SOMs, to develop an adaptive procedure for landscape classification that can be used in a heterogeneous environment of data, data availability, standards, quality, resolution, management, ecology, physiography, and climate to gain a higher-level understanding of landscape processes, so that existing knowledge can be propagated to other domains. The strengths of ANNs, in general, is that they allow for the development of complex, high-dimensional datasets that are distribution free and can handle nonlinear data structures. The procedures developed in and for this research attempt to overcome the problem of using diverse and complex datasets. The Adaptive Landscape Classification Procedure aims to identify nonlinear landscape patterns from a set of high-dimensional spatial data, including terrain morphometry, hydrology, vegetation, land use, soils, and climate, at a variety of spatial and temporal scales.

The specific objectives of this research are as follows:

1) Demonstrate the capability to transfer knowledge from one area or aspect of the landscape to another where knowledge is limited.

2) Improve understanding and linkages between ANNs and geoinformatics.

3) Develop a method for handling diverse and complex data in a spatial environment and provide an alternative method to traditionally used classification methods.

\subsection{The Adaptive Landscape Classification Procedure}

Automated data collection methods have dramatically increased with advances in technology over the past three decades. Despite these advances, it remains difficult and expensive to monitor and understand all aspects of a natural system. Outside of designated research areas such as the H.J. Andrews experimental forest in Oregon, USA or the Dragonja River experimental watershed in Slovania (Sraj et al., 2006), intensive data collection and research are typically focused on small geographical 
areas with a specific focus, such as stream habitat restoration or evaluation of plant community succession. A procedure developed in this research, the Adaptive Landscape Classification Procedure (ALCP), uses available and known spatial and temporal information within a given landscape to establish data patterns and clusters where there are similarities in the data characteristics. The type of data and the magnitude at which the ALCP is used, depends on the area of focus. For instance, clusters of data can be established within a watershed to strictly determine where similar geomorphic characteristics exist. Using a wide array of terrain-based metrics, the ALCP can produce a complex high-dimensional dataset, reduce it to a lowdimension, and determine data similarities using Self-Organizing Maps (SOMs) clustering. The results are useful for understanding hydrologic processes related to terrain, determining the potential for mass-wasting (i.e., landslides), or understanding the sediment transport potential within a watershed. The ALCP also can help determine where to focus site monitoring and/or instrumentation and restoration activities, evaluate the spatio-temporal effects found in inter-annual seasonal variations or long-term climate change, and provide a predictive capability for biotic variables in the landscape. The results of several case studies conducted during this research are reported.

The use of ANNs provides the core capability in the ALCP. The literature suggest that the use of ANNs in the natural sciences has been steadily applied in the past decade, including a number of studies that also integrate Geographic Information System (GIS) capabilities to strengthen the overall process and provide meaningful results (Bacao et al., 2005a; Bryan, 2006; Catani et al., 2005; Dai et al., 2005; Ermini et al., 2005; Hilbert and Ostendorf, 2001; Hsieh and Jourdan, 2006; Joy and Death, 2004; Wang and Sassa, 2006). ANNs are powerful tools that are well suited for solving complex nonlinear classification problems because they enable the discovery and development of previously unknown data inter-relationships and patterns. In addition, ANNs offer "....an alternative to traditional statistical approaches for predictive modeling when nonlinear patterns exist" (Joy and Death, 2004). The input data for an ANN model can be nonlinear, categorically independent, multi-scaled, incomplete, and have mixed-type parameters such as those that might be found in soils, vegetation, hydrology, and terrain-based data (Catani et al., 2005; Dixon, 2005; Hilbert and Ostendorf, 2001). When an ANN model is established using a wide array of input data, it is well suited to being adapted to different scenarios that might be found in different landscape environments. As is demonstrated by the research documented in this report, the ALCP specifically deals with issues of multiple scales 
by using a "spatial container" that captures input data within a defined boundary, derives statistically descriptive metrics of the data, normalizes the data using principal components analysis, and then delivers the results to the SOM for pattern clustering.

\subsection{Report Contents and Organization}

The results of the research study are reported in the ensuing section of this report, as follows:

- Chapter 2.0 describes the background and relevancy of landscape classification and its importance across many disciplines. It discusses various landscape classification approaches and models and reviews commonly used statistical methods such as multivariate regression and $k$ means.

- Chapter 3.0 provides information about ANNs, including some of their capabilities, capacities, and varying model structures and their requirements. Because many types of ANNs exist, each serving different purposes, gaining a broad understanding of their characteristics provides perspective of the core processor of the ALCP chosen for use in this research-the SOM.

- Chapter 4.0 gives a detailed account of the methodology, framework, and mechanics of the ALCP including data requirements, data production supporting multi-scaled, heterogeneous inputs, and software written to support this research.

- Chapter 5.0 demonstrates and analyzes the ALCP on several test- and realworld applications.

- Chapter 6.0 discusses research findings and conclusions and provides recommendations for future research and development.

- Appendix A documents the primary software codes written to support the ALCP. 
- Appendix B provides a detailed listing of multivariate regression equations used to develop streamflow patterns as analyzed in Chapter 5.0 


\subsection{Landscape Classification and Modeling}

This chapter describes the purpose and current methods of landscape classification including non-statistical methods, statistical models and Artificial Neural Networks. The classification methods provided here are intended to give context to what is commonly used and noted in current literature. This research does not attempt to compare the various classification methods largely because these studies can be found elsewhere (see Bacao et al., 2005b; Bryan, 2006; Gomes, 2007; Lin and Chen, 2006; Rao and Srinivas, 2008; Schmidt and Hewitt, 2004).

\subsection{Purpose of Landscape Classification}

Spatial and temporal processes have an explicit cause-and-effect relationship to landscape patterns. These patterns can be detected by observation of biotic or abiotic factors that have the power to influence ecological relationships, hydrological functions, or other natural or anthropogenic-induced processes. A "landscape" is fundamentally composed of a patchwork of possibly recognizable spatial units, which could vary in extension and character depending on the variables used to identify homogeneous areas; typically, these variables involve elevation, morphometry, climate, vegetation, and soils (Bailey, 1995; Bailey, 2004; Turner, 1989). The notion of landscape classification and the determination of homogeneous areas have been important research issues for many disciplines including geography, ecology, hydrology, watershed and water resource management, land-use planning and policy, and environmental management. The multi-disciplinary need for regionalizing the landscape, or dividing it into different domains, resulted in a variety of classification methods and variables as different views were applied and consequent requirements had to be met. Overall, conducting landscape classification is a difficult task largely because of the fuzzy nature of natural-process boundaries and functions and the multiple scales at which these boundaries and/or processes are observed. In addition, it is difficult to capture or recognize hidden and/or unknown process interactions. Clearly, the elements that are brought into a classification scheme can range from very basic to highly complex, depending on the purpose and question(s) being addressed. Regardless of the classification purpose, (e.g., landscape units, species distribution, or demographics), the objective of classification is to reduce the complexity and facilitate the interpretation of the real world by grouping similar elements together and constructing a convenient abstraction from the original 
observations. As depicted in Figure 2.1, a classification process, no matter which method is used, sorts and organizes the input data space into a feature space with some kind of logical ordering and grouping.

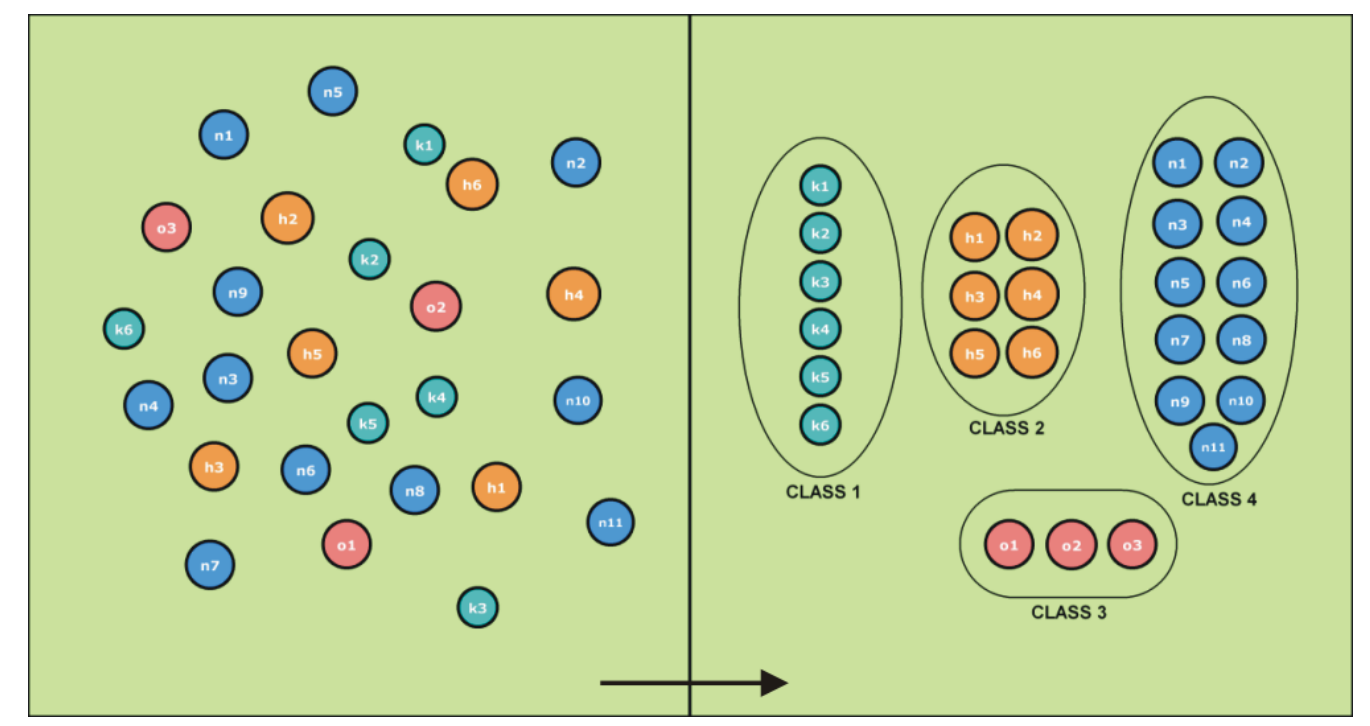

Figure 2.1. The basic process of classification groups univariate or multivariate input data similar or near-similar data into groupings based on a set of rules.

As humans, we have a natural tendency, through normal brain functions, to establish patterns and associations in our environment. It is this ability of pattern recognition that allows us to distinguish objects from one another, to interpret speech, and to read-for instance, to provide meaning to the compilation of letters on this page. Through casual observation, it appears to be relatively effortless to recognize and define basic and homogeneous landscape patterns in our environment such as forest, desert, alpine, grassland, etc. (Watts, 1971). These definitions of broad landscape types introduce an association of further attributes that enhance and unfold the characterization of the environment. For example, through personal experience or knowledge obtained in some other capacity, one might conceive that a desert landscape possesses certain basic characteristics, such as limited water resources, large fluxes in temperature, a limited amount of flora and fauna, etc. These broadly described attributes of the desert landscape have the capacity to reveal new insights and develop relationships between elements within the environment. For instance, because of the large temperature fluxes and limited amount of water, the flora and fauna are dormant in the day and active at night to conserve energy and water; there is a limited amount of vegetation due to a limited amount of precipitation; the hardy structure and composition of desert flora are the result of their protecting themselves in a landscape that has many environmental 
extremes. While the previously described characterization of the desert environment is a relatively simple task for the human mind to process, it is difficult to mimic this type of pattern recognition in a computational context. In other words, how do you get a computer to recognize the difference between a desert and a forest? The importance and purpose of researching a computationally based pattern-recognition and classification system drive the need for a consistent mechanism that is capable of reducing observational data complexity (e.g., multi-spectral sensors; in situ measurements of streamflow, soil moisture, evapotranspiration, and meteorology). This complexity increases dramatically when considering the horizontal and vertical dimensions of space, the time dimension, and a suite of varying observational data.

Forman (1986) describes structure, function, and change as three fundamental characteristics of the landscape. Structure defines the spatial distribution of energy and matter across the landscape, while function describes the interactions and relationships of the spatially distributed energy and matter. Change is defined by any alteration to the structure and/or function over time. The properties of temporal and spatial dimensions in the landscape will have profound effects on understanding and determining patterns and processes in the landscape. Consider, for example, how the potential effects of climate change in the landscape may be evaluated at a time resolution of $\sim 5-30$ years. They would most typically have an effect over a large area, although how the various effects are revealed in the landscape could be defined as a fine-scale problem (i.e., fine detail changes to plant communities and successive change to the landscape ecology). Conversely, for a localized masswasting event that is caused by a short-duration, high-magnitude precipitation event and results in an immediate disturbance, landscape elements should be evaluated on a fine temporal and spatial scale. However, the long-term effects of the masswasting process can have implications over long temporal scales and large areas depending on the sediment transport mechanisms in the landscape and where in the landscape the process is distributed. Human perception of biotic and abiotic processes in the landscape will affect our notion of scale and these perceptions will contribute to the effects of collecting appropriate types and amounts of data to interpret the landscape. These fundamental properties of the landscape provide a basis for understanding the process complexities that exist within it. While it is unlikely that the landscape and its dynamic interactions and processes will ever be fully understood, the use of models to simulate natural processes can assist in bringing complexities to a comprehensible level. 
To evaluate process relationships in the landscape, elements need to be classified over a range of observations, from the micro-scale to the regional-scale and beyond. This type of approach allows for "neighborhood relationships and landscape position in a higher-scale context" (Schmidt and Hewitt, 2004). For example, evaluating many small individual areas for micro-topography elements may miss the bigger picture; e.g., that what you are actually evaluating is an entire mountain. Multi-scaled classification, through space and time, is an important step to reveal different patterns.

\subsection{Current Methods of Landscape Classification}

The methods for classifying landscape currently include non-statistical approaches and statistical models.

\subsubsection{Non-Statistical Approaches}

Traditionally, common and accepted practices in landscape classification have involved direct observations and interpretations of landscape patterns (see Figure 2.2), which were frequently based upon biotic factors (Bailey, 2004; Bryan, 2006; Lioubimtseva and Defourny, 1999; Osinski, 2003). While some approaches were rather simple, abstracting the landscape for broad-area regionalization, other approaches managed the complexity of the natural environment by using a hierarchical approach in which patterns at multiple scales are assumed as controllers of ecosystem functions (Bailey, 1995; Snelder and Biggs, 2002). For example, broad elements of time and space, such as climate, will have the largest control over the landscape having the power to affect water resources, soil composition, land cover, etc. The hierarchical approach ranges from broad macro-processes to microprocesses where each successive element has less control over the environmental condition than the preceding element (Bailey, 2004; Snelder and Biggs, 2002) (see Figure 2.3).

The advent of digital spatial data and GIS technology brought forth the gathering of a multiple datasets where simple or complex integrating and weighting schemes were established, thus revealing information in entirely new ways. For example, long-term mean values of meteorology, vegetation, and soil types could all be assigned a 
unique code based on their attributes. Then, using a raster data model, or a cellbased matrix where each cell contains an attribute value and a position in space, the various data could be combined to create a set of classifications based on the arithmetic sum of the unique codes. These GIS-derived landscape patterns revealed a more complex spatial representation than manually delineated classifications.

These new approaches were the beginning of using computational methods to reveal complexities found in the natural environment. With increases of data availability and spatial resolution came the potential for data errors. This was exemplified with data produced using automated and/or semi-automated collection procedures such as those used in the development of Digital Elevation Models (DEMs) (Russell, 1997). In many cases, GIS approaches had to be supplemented with manual interpretation and delineation of similar landscape types, integrating information from other sources, such as field notes, existing classifications, or other types of information and yielded hybrid approaches in the classification process.

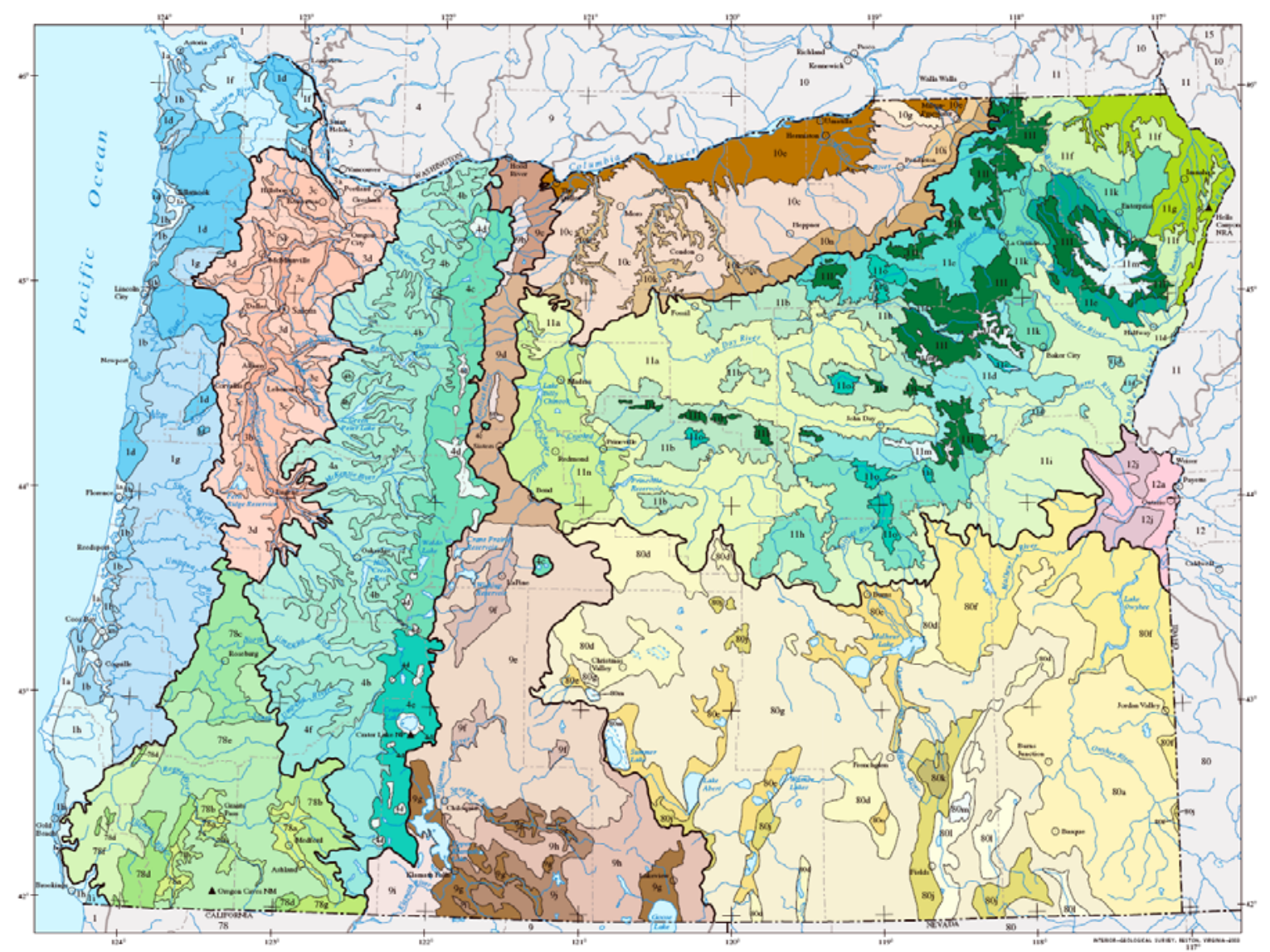

Figure 2.2. An example of an observationally interpreted landscape classification using variables of geology, physiography, vegetation, climate, soils, land use, wildlife distributions, and hydrology (Thorson et al., 2003). 


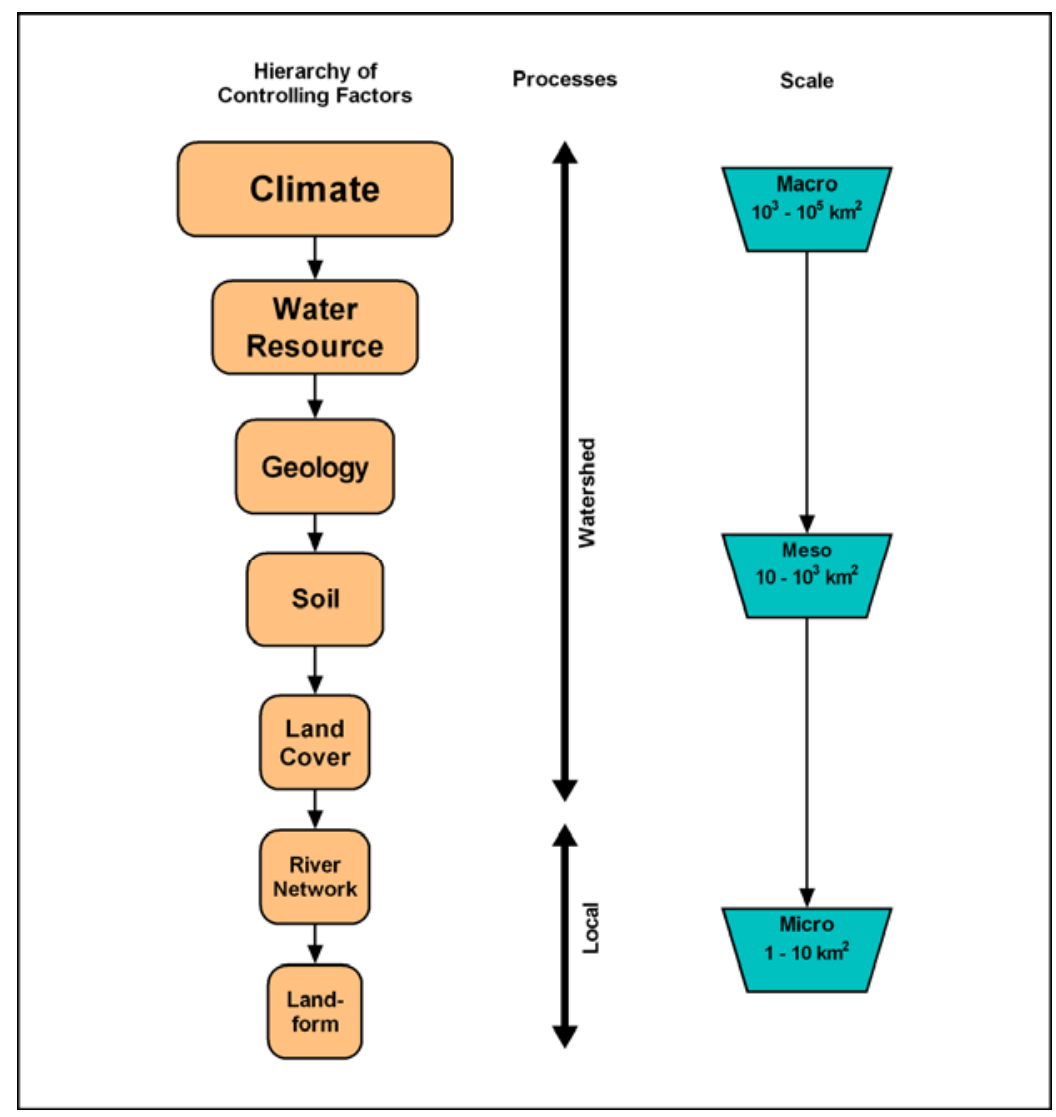

Figure 2.3. Hierarchical approach to landscape classification where elements operating at broad temporal and spatial scale have more dominance over system processes (adapted from Snelder and Biggs 2002).

\subsubsection{Statistical Models}

Statistical techniques have been a common theme in the arena of landscape classification and particularly in the last two decades where they could be applied more easily within a digital geospatial context. The advantages of using various statistical methods helped to provide classifications with stronger bases and quantitative significance with respect to manual interpretations, hierarchical classing, and aggregation and/or weighting techniques. From a statistical point of view, classification problems can be further broken down into three classes (Michie et al., 1994): 1) classic statistical approaches such as linear discrimination and explicit probabilities; 2) machine learning, which employs logic-based automated processing that uses large amounts of data to train into interpretable classes; and 3) ANNs, which mimic the unconscious side of brain function-solving relationships and are incorporating statistical and machine learning methods. A common theme of any classification is the need to apply an objective method for determining the class boundaries. Statistical models such as multivariate regression, $k$-means, linear and 
quadratic discriminate analysis, decision trees, and Bayesian networks are viewed in current literature as being significant for obtaining or generating the classification of data.

A basic clarification needs to be made here, to explain the difference between "classification" and "clustering." The terms are often seen throughout the landscape classification and statistics literature as they will be in this text. A formal classification procedure involves placing processed objects into known or recognized classes. A classic and simple example of classification involves sorting mail into delivery groups based on the mailing address; this is a situation for which there is a clear and defined class boundary. More difficult examples of classification might involve analyzing the spectral signatures of multispectral remote sensing data to determine vegetation classes. This kind of task requires data patterns for training so that the remainder of the dataset can fall into the "appropriate" or "predefined" class boundary. Classification procedures also are commonly found under the terms "pattern recognition," "discrimination," and "supervised learning" (Michie et al., 1994). Common methods for classification include the use of Maximum Likelihood Classifiers, $k$-nearest neighbor, Ward's method, logistic regression, Support Vector Machine, decision trees, and Bayesian networks (Bathgate and Duram, 2003; Caratti et al., 2004; Fritzke and Loos, 1997; Michie et al., 1994; Wardrop et al., 2005).

Clustering methods are often referred to as "unsupervised learning" and involve establishing a structure in the input data providing a basis for groupings or classes of objects. These are cases in which no known or pre-defined classes are in place. Rohwer et al. (1994) state that unsupervised learning "offers the possibility of exploring the structure of data without guidance in the form of class information, and can often reveal features not previously expected or known about." Some methods of clustering data are measured through the computing of dissimilarity between multivariate objects. As a result, objects that have a low dissimilarity are grouped together in the same cluster. These types of clustering scenarios are typically constructed with a matrix of standardized or normalized values and a distance measure (e.g., the Euclidean distance or the city-block/Manhattan distance) is applied to formulate the measure of dissimilarity. Common clustering methods include $k$-means, ISODATA, SOMs, Ward's method, and Principal Component Analysis (Bacao et al., 2005b; Ball and Hall, 1965; Bryan, 2003; Bryan, 2006; Lin, 2006; Mangiameli et al., 1996; Osinski, 2003; Pelleg, 2004). 
A brief review of some of the common statistical classification approaches used in landscape classifications follows. This review is not intended to be exhaustive of all classifiers available, but rather to guide a discussion of the methods in common use with respect to the methods that will be used in this research.

\subsubsection{Maximum Likelihood Classifier}

The Maximum Likelihood Classifier (MLC) is a popular parametric statistical decision rule for classifying multivariate data, often used on remotely sensed multispectral data. Part of the popularity of MLC is due to "its robustness and simplicity" (Yuras, 1996). The MLC is a supervised classification, so it uses a training dataset that contains a relationship between multivariate object properties and known classes. The classes are defined by

$$
\omega_{i}, i=1, \ldots M
$$

where $M$ is the defined number of classes for the data. Three processing steps take place in the MLC: 1 ) the training dataset is used to calculate a mean vector, $m$, of the determined classes and is defined by

$$
m=\frac{1}{M} \sum_{i=1}^{M} x_{i}
$$

where $X_{i}$ is the multivariate object. Similarly, a covariance matrix, $C$, is calculated for the training data and is defined by

$$
C=\frac{1}{(M-1)} \sum_{i=1}^{M}\left(x_{i}-m\right)\left(x_{i}-m\right)^{T} \text {; }
$$

2) a distance to the training class mean, $m$, is determined for each multivariate object in the dataset; and 3) a probability of membership, using the probability distribution function

$$
g_{[m, C]}=\frac{1}{(\sqrt{2})^{n} \sqrt{\operatorname{det}(C)}} e^{\frac{-(x-m)^{T} C^{-1}(x-m)}{2}}
$$


is assigned and each object goes to the class with the highest probability of containing it which is defined by the decision rule

$$
x \in \omega_{i,} \text { if } p\left(\omega_{i} \mid x\right)>p\left(\omega_{j} \mid x\right) \text { for all } j \neq i
$$

where $p\left(\omega_{i} \mid x\right)$ is the probability of a given object belonging to a given class (Evans, 1998; Richards and Xiuping, 2006).

Results from the MLC appear to be reasonable in many applications (Bathgate and Duram, 2003; Shanmugam et al., 2006; Short, 2006; Stow et al., 2007; Vrieling et al., 2007); however, the MLC has some general limitations as follows. First, the training data must a have a Gaussian, or normal, distribution that signifies a certain degree of homogeneity in the data. Second, because the MLC is a supervised classifier, training sets are required to classify the objects, thus a priori knowledge is required. As is generally the case with statistical sampling, the more training sets that can determined, the greater likelihood of a higher accuracy classification.

\subsubsection{Multivariate Regression}

Multivariate regression is a commonly used statistical model for classification and prediction tasks. Its basic function combines multiple independent variables to determine a single dependent variable, taking the form:

$$
Y=A+\beta_{1} X_{1}+\beta_{2} X_{2}+\ldots+\beta_{n} X_{n}+E
$$

where $Y$ is the predicted value, $A$ is the $Y$ intercept, $\mathrm{X}_{n}$ are the independent variables, $\beta_{n}$ are the coefficients of the independent variables, and $\mathrm{E}$ is an assigned error term.

The model has advantages in being straightforward to use, working to develop a relationship between the variables, and providing a goodness-of-fit estimate for easily evaluating results (i.e., chi-square test, coefficient of determination/correlation coefficient). With the simplicity of the model, Wetherill (1986) emphasizes caution related to the easy misuse of the regression procedure. Some known issues with multivariate regression include known relationships in the data often not being 
detected, noisy data yielding incorrect results, and the general approach of multivariate regression being a better fit for linear data, which are not typical in the natural environment (Caratti et al. 2004).

\subsection{5 k-means}

The $k$-means (MacQueen, 1967) exhibits a non-hierarchical, unsupervised, multivariate clustering model that does not assume an input data distribution, any measures of dissimilarity, nor a class structure. The standard $k$-means iteratively selects random "seeds" in the existing dataset and tests for its center position in the cluster or partitioning as defined by the parameter $k$ (see Figure 2.4). It is after each iteration that the corresponding cluster center is adjusted, the Euclidean distances of the data objects are calculated, and cluster membership is defined for each object. The algorithm converges when there is no more movement in each of the determined cluster centers ( $\operatorname{Lin}, 2006$ ). The algorithm works to minimize a squared-error function defined by MacQueen (1967):

$$
J=\sum_{j=1}^{k} \sum_{i=1}^{n}\left\|x_{i}^{(j)}-C_{j}\right\|^{2}
$$

where $x_{i}^{(j)}$ is an object in the input set, $c_{j}$ is the current cluster center, and $\left\|x_{i}^{(j)}-c_{j}\right\|^{2}$ defines the distance measure.

The $k$-means model may have difficulties in finding cluster centers with larger nondistinctive datasets and may not always find the optimal center of the cluster (Lin, 2006). Repeated execution of the $k$-means model on the same dataset yields different results because of the randomness of the starting "seeds." This issue can be overcome by performing a series of runs and selecting the solution that appears most often. Depending on the situation, an advantage and/or disadvantage of $k$ means is the lack of enforcement to determine the number of clusters; the model relies on defining natural clusters that define the feature space. However, using the Lloyd algorithm in conjunction with $k$-means provides the ability to define a set number of clusters (Lloyd, 1982). 


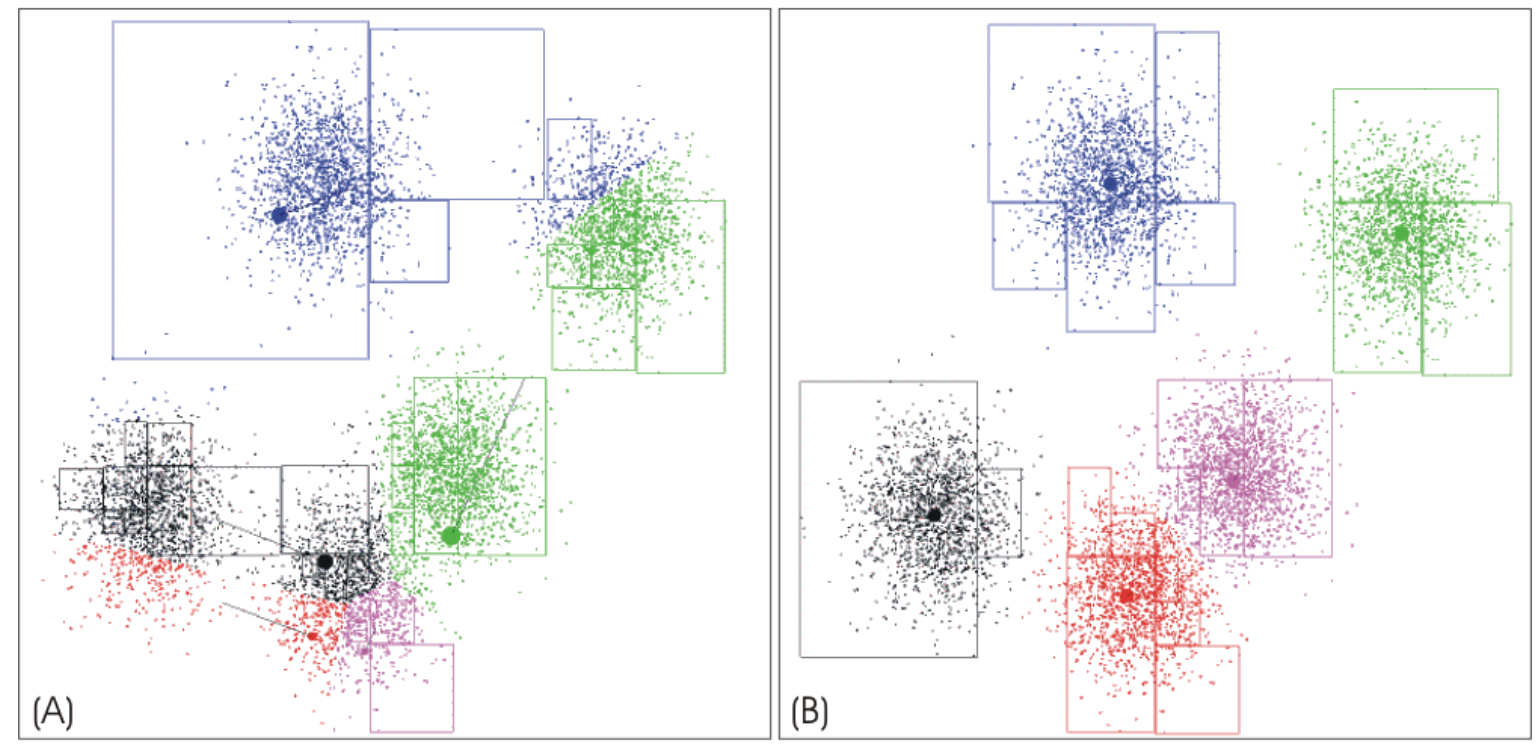

Figure 2.4. A sample of 8000 points in the initial phase of k-means processing with random starting "seeds" placed in the input data space (a) and the final convergence stage (b). The cluster center, $C_{j}$, is indicated by the large colored dot and parameter $\mathrm{k}=5$ resulting in five distinctive cluster areas (Pelleg, 2004).

Other variants of the $k$-means model that are used in multi-dimensional classification include ISOCluster and fuzzy k-means. The ISOCluster model (Richards, 1986), or Iterative Self-Organizing Clustering, uses the central idea of $k$-means, updating cluster centroids until minimal distances are reached (i.e., convergence), with the well-known ISODATA model (Ball and Hall, 1965) and the MLC (see Figure 2.5). ISOCluster requires the number of clusters to be defined; however, if a more freeform, natural clustering approach is needed, it is possible to set a high cluster number (i.e., the parameter $k$ ) and then "aggregate clusters after interpretation" (Eastman, 2006). 


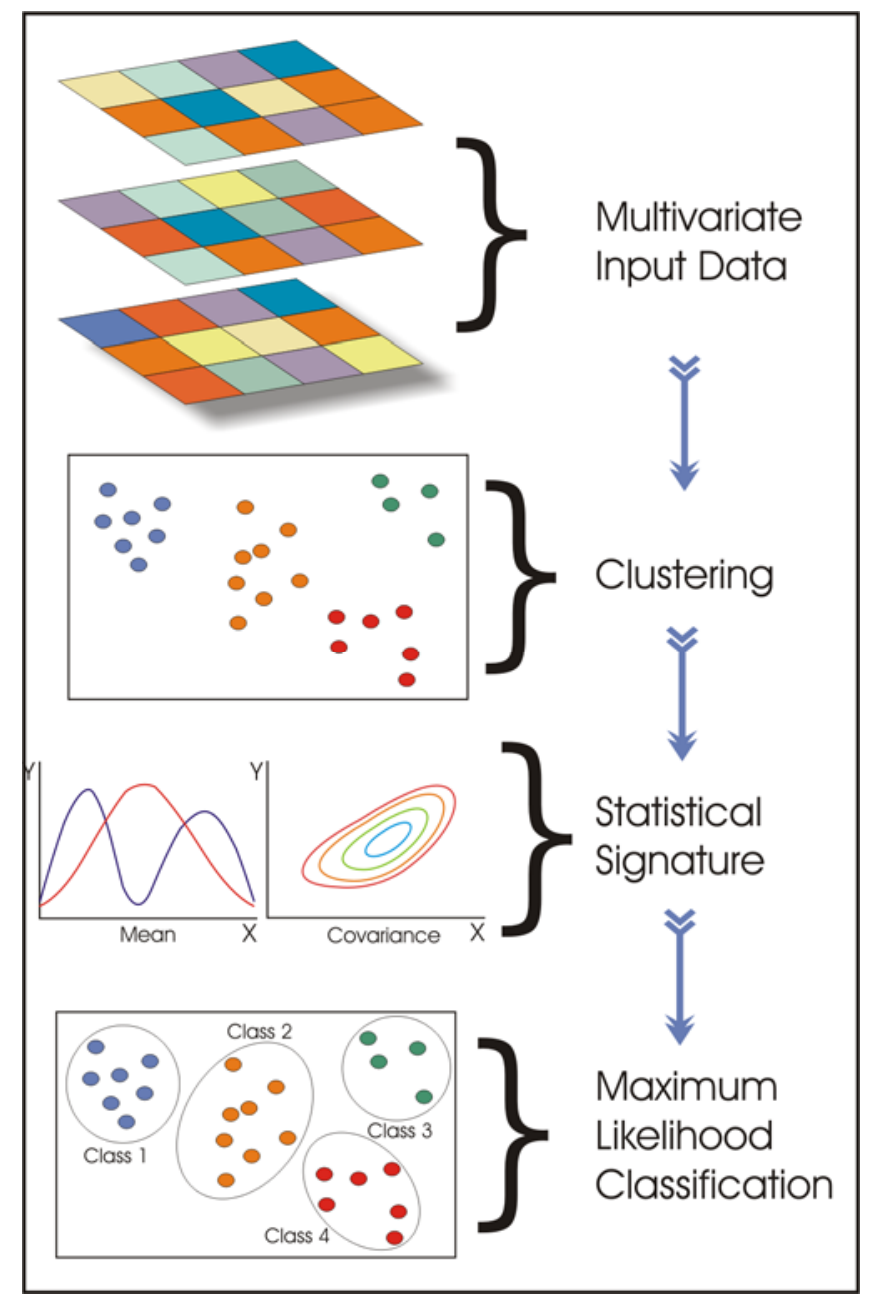

Figure 2.5. The ISOCluster model incorporates $k$-means, ISODATA, and the Maximum Likelihood Classifier, to organize and classify multivariate data.

ISOCluster is an unsupervised multivariate model that is commonly found in the literature and is readily available in most image-processing and GIS software. As is the assumption with the MLC, ISOCluster also assumes that the input data follow a normal distribution. In some cases, data can be transformed into a normal distribution by running a log-transformation (Ziadat, 2005).

Fuzzy k-means (DeGruijter and McBratney, 1988) is very similar to the standard $k$ means model; the major difference is the application of the fuzzy-set theory allowing a degree of membership in multiple cluster sets. This model has been used in various research and appears to be gaining momentum in its application (Bolliger, 2005; Burrough et al., 2001; McBratney and DeGruijter, 1992; Minasny and McBratney, 2002; Schmidt and Hewitt, 2004). Fuzzy k-means uses a similar iterative minimization of the sum of square errors as standard $k$-means, but uses a term for fuzzy membership, or the idea that a data object can belong, with varying degrees, to 
more than one defined class. The model is defined by Minasny and McBratney (2002), as follows:

$$
J=\sum_{i=1}^{n} \sum_{k=1}^{c} m_{i k}^{\phi} d^{2}\left(x_{i}, c_{k}\right)
$$

where $n$ is the number of input data, $c$ indicates the number of classes (equivalent to $k$ in $k$-means), the exponent $\phi$ is the fuzzy membership parameter that can range from $1-\infty, X_{i}$ is the individual input data, $C_{k}$ is the centroid of $k=n$, and finally,

$d^{2}\left(x_{i}, C_{k}\right)$ is the squared Euclidean distance between the data object and the class centroid. The fuzzy membership parameter, $\phi$, produces a hard and discrete cluster boundary at a minimum value of 1 and increases the degree of fuzziness as the parameter approaches infinity, and it ultimately leads to the data object set to being assigned to a single class.

In general, fuzzy-set based models for landscape classification offer an improvement in terms of understanding the non-discrete boundaries that exist in natural processes. However, difficulty arises in determining ideal fuzzy parameter values that 1) don't over-simplify the landscape with large fuzzy classes resulting in minimal class distinction, and 2) provide enough realism and balance such that class membership is not forced by strict boundaries or defined purely by the data objects. Bolliger and Mladenoff (2005) provide a recommend that $\phi$ to range from $1.2-1.5$ for landscape classifications. An advantage to using a fuzzy $k$-means approach is gaining an assessment of the uncertainty found in the data classes (Burrough et al., 2001; Schmidt and Hewitt, 2004).

\subsubsection{Ward's Hierarchical Clustering}

A statistical model commonly used in landscape classification is Ward's (1963) agglomerative hierarchical clustering (Bolliger, 2005; Bolliger and Mladenoff, 2005; Lin, 2006; Osinski, 2003; Wardrop et al., 2005). The model groups the input data in an iterative bottom-up (i.e., agglomerative) style, where in the first processing step all data points, $j$, make up their own individual clusters, $i$, such that $j=i$. In a hierarchical form, two data points that are most similar are grouped and the process is iterated until there is only a single cluster remaining. Ward's clustering differs from 
other clustering models in that it does not use a distance metric such as Euclidean or city-block, but rather a measure of minimum variance. All data, $j$, are evaluated for their error sum of squares, which is a measure of information loss, and is defined by

$$
E S S=\sum_{i} \sum_{j} \sum_{k}\left|X_{i j k}-\bar{x} i \cdot k\right|^{2}
$$

where $X_{i j k}$ represents the value for variable $k$ in data $j$ within a given cluster, $i$ (Wiesner, 2008). A pair of data with the minimal error sum of squares creates the first clusters in the hierarchy. The evaluation of the minimal error sum of squares is repeated in the second processing step, but instead of evaluating individual data point clusters, the cluster means that contain a larger data membership are used until the final cluster is formed containing all data points. The result is something resembling a tree, formerly known as a dendogram (see Figure 2.6).

While agglomerative hierarchical clustering (i.e., Ward's clustering) is a popular choice for many applications, including landscape classification, it has clear limitations. First, it is not possible to determine the number of natural clusters in the data; instead, these must be defined with a priori knowledge, if available.

Additionally, Mangiameli et al. (1996) state that "to obtain the best cluster results, the investigator must have considerable knowledge about the empirical data including the number of natural clusters, the statistical distribution of observations within the natural clusters, the presence of outliers, and the density of observations among the natural clusters. The information required for an intelligent choice of cluster heuristic is usually not available." Lin and Chen (2006) also address biasing: "Ward's method tends to join clusters that contain a small number of sites, and it is strongly biased when the clusters have roughly the same number of sites." Ward's clustering, however, is well suited to handle large multivariate datasets and stands out among other hierarchical clustering models because it uses a minimum variance rather than a distance metric. 


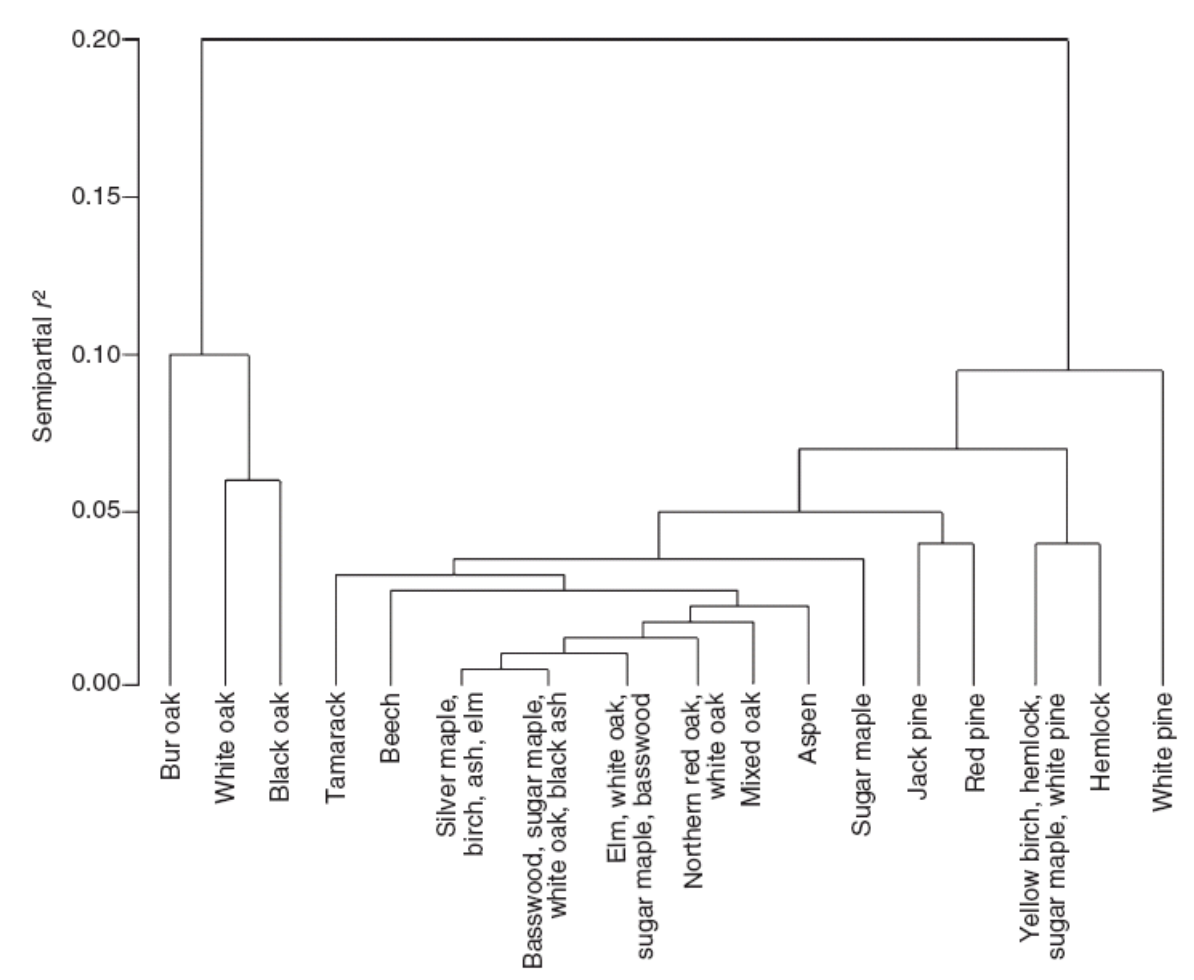

Figure 2.6. An example of the agglomerative hierarchical clustering for dominance of tree species in Wisconsin, USA (Bolliger et al., 2004).

\subsubsection{Artificial Neural Networks}

Artificial Neural Networks have been used in landscape classification analyses (Bacao et al., 2005a; Bryan, 2006; Ehsani, 2007; Hilbert and Ostendorf, 2001; Hsieh and Jourdan, 2006; Joy and Death, 2004; Lenz and Peters, 2006; Park et al., 2001), but they are not as commonly used as the other models previously discussed. Potential reasons for this may be the complexity of the process, the number of parameters that need to be tuned, the many different types of ANNs, and the mixed results that have been published (i.e., about ANNS being found to be useful or not useful). ANNs show their strength and agility in handling complex, nonlinear, distribution-free, high-dimensional datasets. The great variety of ANNs developed to date includes the popular Multi-Layer Perceptron (MLP) network, the Radial Basis Function (RBF) network, the Recurrent Neural Network (RNN), and the Adaptive Resonance Theory (ART) network. ANNs have been used in the remote-sensing field for many years in a research mode (Atkinson and Tatnall, 1997; Civco, 1993; Richards and Jia, 1999; Tso and Mather, 2001), and they are beginning to find a place in commercial remote-sensing software (Eastman, 2006). Additionally, ANNs are used in some GIS analyses, but the processing steps are loosely coupled. To 
the author's knowledge, the only coupled commercial or open-source GIS/ANN implementations are 1) ArcSDM (Spatial Data Modeler) (Sawatzky et al., 2004), which focuses on mineral exploration but can be used for other applications in which a spatial prediction is required, and 2) the JDEVS (Java Discrete Events System) (Filippi and Bisgambiglia, 2004), which provides an environmental modeling framework that links GIS and ANNs. The study reported here focused on the use and application of the unsupervised ANN, SOMs. Further detail on ANNs and specifically, SOMs, is provided in the following chapters. 


\subsection{Artificial Neural Networks}

Because the objectives of this research are focused mainly on the pattern recognition capabilities of ANNs, it is necessary to 1) understand their varying capability and benefit for pattern recognition, and 2) understand the benefits and limitations of their use in classifying the landscape. This chapter provides a basic understanding of pattern recognition, soft computing, ANN models used in classification, and a more detailed description of SOMs, which are the core ANN model used in this research. A hierarchical approach is used to explain and define how SOMs fit into the bigger realm of soft computing and how patterns can be used to classify data. Under the broad umbrella of soft computing, ANNs offer a large array of resources to apply to an even larger number of possible application areas. In general, ANNs capable of solving classification problems can be categorized into "supervised" and "unsupervised" ANNs. SOMs offer a well-recognized ability to handle unsupervised classifications on large complex datasets. The concepts, procedures, algorithm, and some of the mathematics of the SOM are presented. Finally, a simple demonstration of the SOM using a red-green-blue (RGB) colorset illustrates how a complex and randomized dataset can be organized and reduced in its dimensionality. While the colors used in the demonstration make it easy to see and understand the strength of the classification, the use of and potential for a SOM to reduce and classify nonlinear multivariate data from the landscape must also be considered.

\subsection{Pattern Recognition}

As discussed by Bishop (1996), "pattern recognition encompasses a wide range of information processing problems of great and practical significance." Chapter 2.0 of this report introduced some of the basic concepts of landscape classification and further concepts will be considered here to emphasize the importance of pattern recognition and the role it plays in ANN processing.

Brain functions can, seemingly with relatively little effort, distinguish objects in the surrounding environment. The characteristics of the objects include color, shape, texture, smell, etc., all of which help us to distinguish and capture, at varying levels of detail, their function and state. The task of performing pattern recognitions in a computational setting is one that has represented a scientific challenge for decades and has become the focus of Artificial Intelligence. To grasp the simple pattern 
recognition capabilities of the brain, consider the pattern examples shown in Figure

\section{1 through Figure 3.3 (SFCC, 2007).}

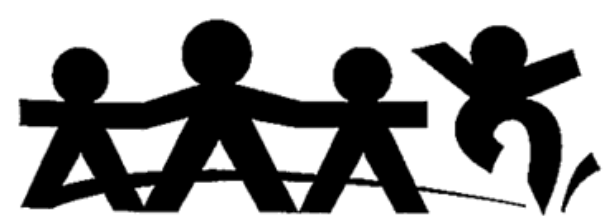

Figure 3.1. This illustration exemplifies an anomaly (far right) in a regular pattern space. The mind immediately picks up on the abnormality, which thus becomes a point of interest (SFCC, 2007).

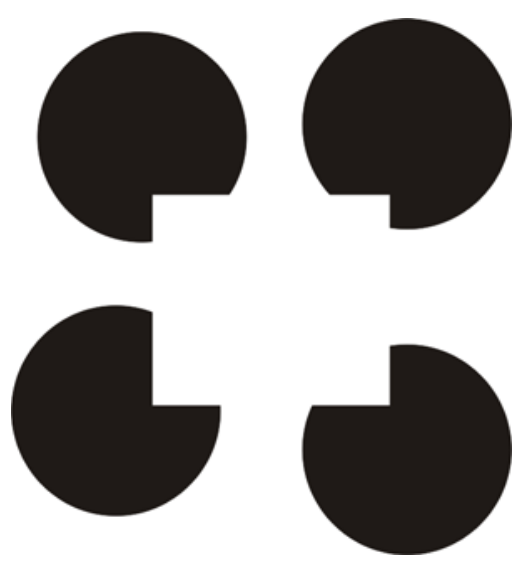

Figure 3.2. This set of objects relates the brains' natural ability to recognize patterns and fill in missing information. Note the only objects that actually exist in this illustration are four incomplete circles with varying amounts of missing information. Through pattern-recognition, four complete circles and a square are comprehensible (SFCC, 2007).

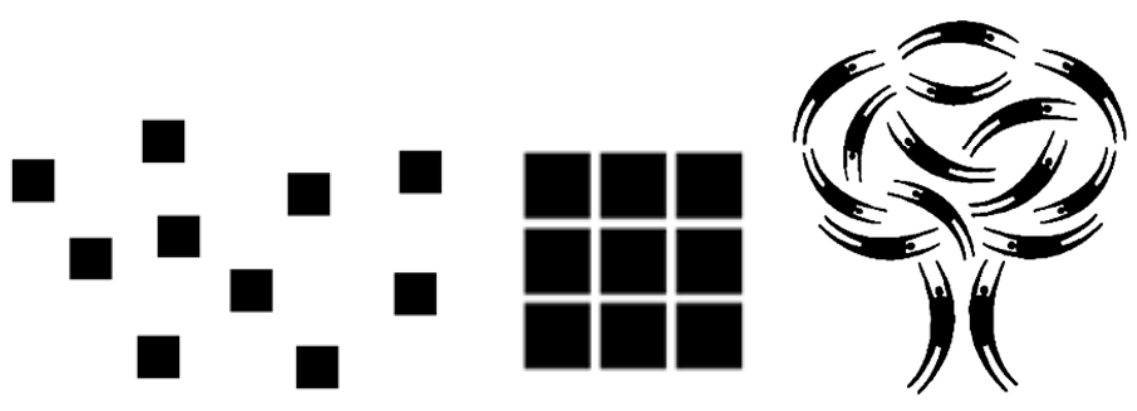

Figure 3.3. This illustration exemplifies the concept of proximity where the pattern on the left is viewed as a series of separate objects, the one in the middle is viewed as a single object (although it consists of separate objects), and the one on the right is viewed holistically as a single complex object composed of similar objects with different orientations (SFCC, 2007).

The examples illustrated can be related back to actual multivariate patterns and to the challenges related to incorporating pattern recognition in a machine-learning context. In the case of processing imagery for pattern recognition, the data are processed as a multi-dimensional matrix. Interestingly, the three sets of patterns 
shown in Figure 3.1 through Figure 3.3 are rooted in the early $20^{\text {th }}$ century work by a group of German psychologists who identified the ability of the human brain to pattern in various modes, and established "mental laws" referred to as the Gestalt Principles. Consider for a moment, the capability of the human brain to process a single day's worth of information and logistics as well as its adaptive nature for survival. The brain has the ability to "process millions of visual, acoustic, olfactory, tactile, and motor data, and it shows astonishing ability to learn from experience, generalize from learned rules, recognize patterns, and make decisions." (Kecman, 2001). The science of Artificial Intelligence (AI) works to mimic the brain's massive capability.

\subsection{Soft Computing}

Artificial Neural Networks are part of a larger field of study under the overarching topic of "soft computing." Support Vector Machines, evolutionary and genetic algorithms, swarm intelligence, and fuzzy logic models also can be included in soft computing. These computational models were largely developed to deal with the complexities and unknown boundaries of large multivariate datasets. Kecman (2001) refers to soft computing methods as "universal approximators of any multivariate function... of particular interest for modeling highly nonlinear, unknown, or partially known complex systems, plants, or processes." The complexities observed in the landscape exhibit characteristics for which soft computing is well suited. The notion of soft computing is contrary to "hard computing" methods where strict logic is used to achieve a definitive and precise answer. The paradigm of soft computing addresses several characteristics of uncertainty in the landscape, including 1) observations and data representations; 2) understanding of physical and biological processes; and 3) our model representations of landscape processes. The acknowledgement of these uncertainties, in addition to the fuzzy nature of the landscape provides an argument for using soft computing methods to better understand and represent the landscape. The following sections focus on ANNs to further the conceptual understanding of these computational models for application to landscapes.

\subsection{Fundamental Basis of Artificial Neural Networks}

The initial concept of ANNs as dealt with by McCulloch and Pitts (1943), who formalized the concept of an artificial neuron. Work on ANNs was relatively non- 
existent until the early 1980s, when Hopfield (1982) set the stage for using ANNs in a wide array of multi-disciplinary fields.

A formal definition of an ANN model is provided by Aleksander and Morton (1990):

"A neural network is a massively parallel distributed processor that has a natural propensity for storing experimental knowledge and making it available for use. It resembles the brain in two respects:

1) Knowledge is acquired by the network through a learning process.

2) Interneuron connection strengths known as synaptic weights are used to store the knowledge."

ANN models work to mimic the functions of neuron cells in the brain. A basic representation of the neuron and its interaction with other neurons is presented in Figure 3.4. Communication of information among the brain's neurons is conducted through electrical pulses of various frequencies and magnitudes. These electrical pulses are produced as a response to the input of information into and within the body and allow communication not only within the brain but with all body functions (i.e., muscle response, organ function, etc.). The primary components of the neuron are the nucleus, dendrite, axon, and synapse. In general, dendrites are responsible for receiving information from other neurons, the axon sends an electrical signal (i.e., information) to neighboring cells, and the synapse is the connection point between an axon on one cell and a dendrite from a neighboring cell.

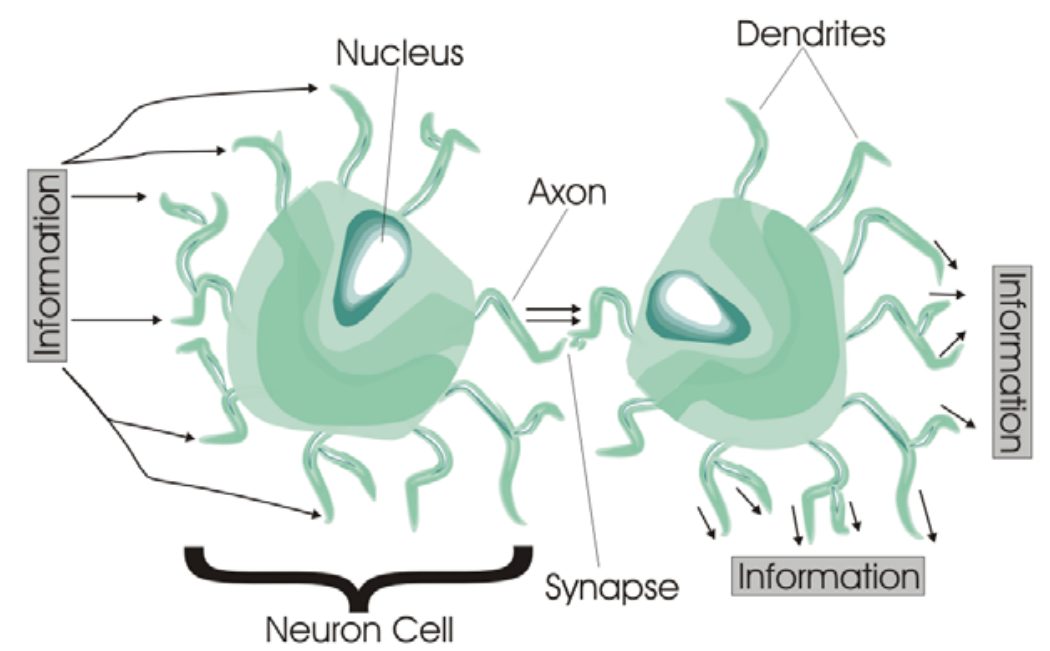

Figure 3.4. A simplified graphic representation of a neuron cell processing and transmitting information from cell to cell (adapted from Lingireddy and Brion, 2005). 
Neurons function in a massively parallel manner as signal communications occur across many cells, and each cell can have as many as 10,000 dendrites that are continually being fed electrical signals (Smith, 1996). The many sources of signals being fed into an individual cell are resolved into a single signal, which is output through the axon and delivered to associated dendrite receptors. A schematic representation of the neuron cell communication via an ANN is presented in Figure 3.5. ANN models retain some of the neurological vocabulary to describe the components of the system. As represented in Figure 3.5, the spheres are "neurons" and the lines connecting the neurons are "synapses." In real neuron cells, the synapses inflate and contract to control the sequence of the signals in terms of their magnitude and frequency. The synapses in an ANN model function similarly where "connection weights" along the individual synapses are adjusted and optionally, imposed delays are applied to assist in the learning process. The hidden layer shown in Figure 3.5 provides an "activation function," which transforms the sum of the input data into a data signal using one of many possible functions, including logistic (sigmoid), hyperbolic, exponential, sine, and square root. Table 3.1 is a comprehensive list of activation functions. Increasing the number of hidden layers allows the neural network to handle even more complex problems; however, caution must be exercised so as to not over-commit the input data, thereby causing generalizations (Lingireddy and Brion, 2005; Rohwer et al., 1994).

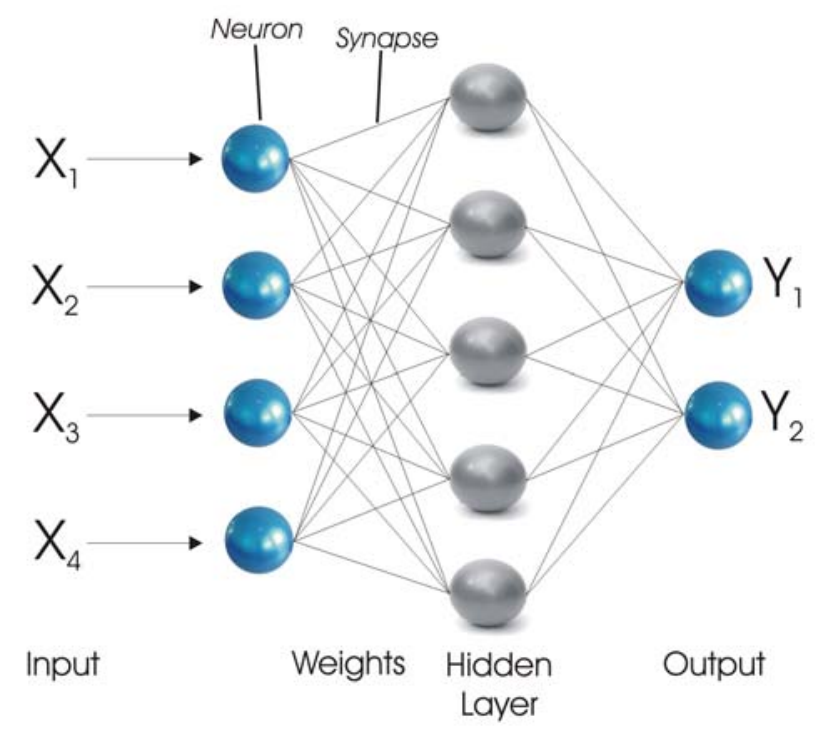

Figure 3.5. A common and simple ANN schematic that represents the flow of information from the input data, to the receipt of the input neurons, to weighting and evaluating of data, to signal adjustments in the hidden layer, and finally the resulting output data (adapted from Principe et al., 2000). 
In a supervised ANN classification, the input data are compared to a set of training data that defines "truth" in the data relationships. The error of the solution is obtained with each iteration, and the connection weights are adjusted (increased or decreased) according to how the output data compare with the training data. The ultimate goal is to determine the combination of connection weights that provide the minimum error between output and training data. The weighting process is used as a probability measure where weights are increased to represent higher probabilities and are decreased for weaker probabilities. Therefore, as input data signals are presented to the ANN, the strongest probability and most frequent occurrence of the signal can be grouped with others. This process is repeated until the optimal solution with the various combinations of connection weights is determined. The optimal solution, often referred to as a "trained ANN" or "trained net," can then be used to classify future input data where the class isn't known. A common approach used to assess a trained net is to use two-thirds of the existing training data for establishing the neural network, then run the remaining one-third of the training data through the model to evaluate how capable the model is at making the predictions. 
Table 3.1. Common activation functions used in ANN models (StatSoft, 2003).

\begin{tabular}{|l|c|c|}
\hline Function Name & Function $f(x)$ & Data Range \\
\hline Identity & $x$ & $-\infty,+\infty$ \\
\hline $\begin{array}{l}\text { Logistic } \\
\text { Sigmoid) }\end{array}$ & $\frac{1}{1-e^{-1}}$ & $(0,+1)$ \\
\hline Hyperbolic & $\frac{e^{x}-e^{-x}}{e^{x}+e^{-x}}$ & $(-1,+1)$ \\
\hline Exponential & $\frac{e^{-x}}{\sum_{i} x_{i}}$ & $(0,+\infty)$ \\
\hline Softmax & $\frac{x}{\sum_{i} x_{i}}$ & $(0,+1)$ \\
\hline Unit Sum & $\sqrt{x}$ & $(0,+\infty)$ \\
\hline Square Root & $\begin{array}{l}\text { sin }(x) \\
x-1<x<+1 \\
+1 x \geq 0\end{array}$ & $(-1,+1)$ \\
\hline Sine & $\left\{\begin{array}{c}-1 x \leq 1) \\
\text { Ramp }\end{array}\right.$ & $(0,+1)$ \\
\hline
\end{tabular}

ANNs are considered semi-parametric classifiers because they use both parametric discriminate functions and nonparametric shape discriminators. The combination provides a unique and dynamic approach to traditional statistical classifiers (Principe et al., 2000). A common use of ANN models seeks to solve regression, classification or pattern recognition, prediction, and signal processing problems, all of which routinely are used as components of decision support systems. While ANNs have their foundations in conventional statistical models, they differ in that 1) Gaussian, or normal, distributions of data are not required; 2) linear or nonlinear data are acceptable for inputs; 3) adaptive learning is an integral part of the model; and 4) there is a high degree of error tolerance that can throw off results in common statistical methods, provided a reasonable signal-to-noise ratio exists in the data. ANN models make no assumptions about the input data, other than they expect numbers, not alphanumeric characters, and will adjust the weights of the internal 
network directly from the input data. As stated by Perus and Krajinc (1996), "the most important thing is that ANNs allow a different view of problems which cannot be solved by [exact] statistical methods due to their theoretical limitations." In addition, natural data cannot always be described with low-order statistical parameters, because these elements and relationships between elements can exhibit nonlinear associations. For this reason, the adaptive characteristics of ANNs are more effective and allows for an automatic determination of parameters through an evaluation and repeated weighting adjustment of the input data space (Kohonen, 2001). In the most commonly used ANNs, the use of "feedback loops" provides an adaptive capability and enables ANNs to evaluate their own performance, which is often a measure of mean square error of model prediction value and the targeted or trained value (see Figure 3.6). The performance values are used to continually adjust the parameters of the ANN to match some known condition, allowing for learning and training to improve upon the output results. The idea of performing continual adjustments introduces the notion of "adaptive learning" and is especially effective for data that continually evolve in time and space, such as meteorology, vehicle traffic, disease spread, and landscape processes.

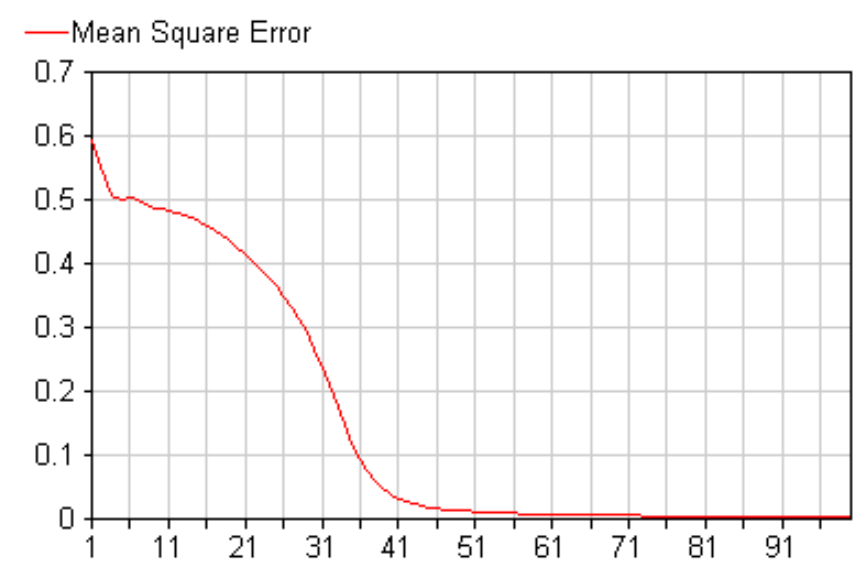

Figure 3.6. Result of a simple multi-layer perceptron ANN evaluating the MSE over model iterations. Once a sufficient MSE is reached, the optimal solution is obtained, meaning ANN output values closely match the training values and an underlying relationship has been established between input data vectors and resulting output.

The adaptive learning concept found in ANNs provides a significant advantage over traditional statistical techniques; however, ANNs do have some known disadvantages. First, it is possible to present too much training data to an ANN (too much of a good thing), in which case the model is "over-trained" and crosses a threshold that causes a degradation in model performance by increasing mean square errors. Next, ANNs can have some difficulty in classifying a given data 
pattern where no appropriate class exists. In such cases, the "out-of-class" data are lumped with the category of data that is closest or most similar. While ANNs have proven themselves in a wide array of applications, including hydrologic forecasting, a common criticism is that they are often used as a "black box model," for which input is presented and output is generated, and no understanding of the internal mechanics are required. As is commonly the case with hydrologic modeling, for instance, a physically based model is constructed to transport and explain the movement of water through each component of the hydrologic cycle. The governance of strict mathematical bounds is used to mimic the process of water transport through its various routes (e.g., overland flow, groundwater, evapotranspiration, precipitation, etc.). With an ANN, the detailed physically based processes are effectively lumped into the "black box" and the determined driving input parameters to water transport (e.g., precipitation, temperature, relative humidity, soil moisture, vegetation, and soil type, etc.) are presented along with known outputs (e.g., stream outflow), and thus the ANN determines the data relationships between the input and output without needing knowledge of the flow transport physics.

\subsection{Common Types of Artificial Neural Networks}

Numerous types of network structures are found in ANNs, each possessing a unique behavior and purpose. In some cases, multiple network structures are used together to take advantage of their respective strengths. The topology of the ANN dictates the type of network and the number and shape of the activation functions. This section briefly discusses supervised and unsupervised ANNs used for classification, that are distinguished by the use or no use of training data, respectively.

\subsubsection{Supervised Artificial Neural Networks}

Most ANNs fall under the supervised category. A supervised ANN, by definition, uses a training dataset for which both the input data and resulting output data are known. The supervision process defines relationships between input and output data by iteratively adjusting connection weights and reducing model errors. This adjustment occurs both at the individual neuron scale and globally through the model, attempting to reduce the model error over time. 
A classical example to explain the ANN training process is Fisher's iris dataset (Fisher, 1936). This dataset captures multivariate information for three known species of the iris flower: 1) setosa, 2) virginica, and 3) versicolor. The measured variables of the iris flowers, as shown in Table 3.2, include 1) petal width, 2) petal length, 3) sepal width, and 4) sepal length. For each species, 50 samples were measured. While Fisher used this dataset to develop a linear discriminant model that helped to identify the flower species based on the physical measurements, it also is a good representation and sample dataset to use to explain what is required for a training dataset in a supervised ANN model. Additional and more comprehensive datasets can be found on the website of the University of California's Irvine MachineLearning Repository: http://archive.ics.uci.edu/ml/.

Table 3.2. A small sample of Fisher's multivariate Iris flower dataset (Fisher, 1936).

\begin{tabular}{|l|l|l|l|l|}
\hline Sepal Length & Sepal Width & Petal Length & Petal Width & Species \\
\hline 5.1 & 3.5 & 1.4 & 0.2 & setosa \\
\hline 4.9 & 3.0 & 1.4 & 0.2 & setosa \\
\hline 7.0 & 3.2 & 4.7 & 1.4 & versicolor \\
\hline 6.4 & 3.2 & 4.5 & 1.5 & versicolor \\
\hline 6.3 & 3.3 & 6.0 & 2.5 & virginica \\
\hline 5.8 & 2.7 & 5.1 & 1.9 & virginica \\
\hline
\end{tabular}

The requirements for a supervised neural network can be substantial in terms of generating a large enough training dataset to capture the variability of the input data. The ANN learns to recognize the variable conditions and adapts itself to respond appropriately. It is ideal to have a large enough cross section of input data to capture the data variability so, while the ANN model is training, it can establish an effective solution of network weights and allow intelligent output if the system encounters data conditions not previously known. Establishing a supervised ANN model throughought the training phase can be time consuming and require tuning of parameters in the model. Parameters such as learning rate, step size, weight decay, momentum rate, synapse delays, beta/gamma conscience, memory, and more are used to assist in the model learning.

\subsubsection{Multi-Layer Perceptron}

The most commonly used supervised ANN model is the feed-forward Multi-Layer Perceptron (MLP). The MLP is linked with the back-propagation learning algorithm, which allows for the iterative adjustment of weights to correct errors in the model (Rumelhart et al., 1986). This neural network is general in nature, allowing for its use in a wide variety of applications including pattern recognition (Ermini et al., 2005; 
Freeman and Skapura, 1991). The feed-forward component of the MLP states that the output vectors produced by the ANN are a function of the input vectors and some weighting process; this is an important distinction to make because variability exists between networks (e.g., Recurrent Neural Networks). MLPs are best suited for pattern recognition and/or classification type problems.

The basic structure of the MLP, shown in Figure 3.5, includes the input layer, one or more hidden layers, and an output layer. The input layer is fed with vectors of data, also termed "codebook vectors," and, in the training phase, a related output value for each codebook vector is offered. The hidden layer is variable in the number of neurons available; thus, depending on the complexity of the problem, more neurons may provide a better solution. However, excessive placement of neurons in the hidden layer can also over-fit the model, thereby creating degraded results.

The MLP exhibits three important characteristics. First, the network is massively parallel and massively interconnected where each neuron in each layer connects to every other neuron in the next layer. Second, the neurons process data in a nonlinear state and pass codebook vectors through a hyperbolic or logistic (sigmoid) type of activation function (see Table 3.1). Third, the final classification of data is resolved by dividing the pattern space using hyperplanes (StatSoft, 2003). As stated by Ermini et al. (2005) the role of the MLP "is not to fit the observed data but to model a process by generalizing the learned experience to other cases not represented in the training database."

\subsubsection{Radial Basis Function Neural Networks}

Radial Basis Function (RBF) neural networks are based on nonlinear regression and classification networks, which differ from the MLP network on several fronts. The classification in the pattern space is defined by spheres, such that data is classified by the radius of each data point from the center of the data distribution. These networks exhibit a single hidden layer and are typically composed of a very large number of neurons. These neurons apply a nonlinear Gaussian surface activation function that is then fed to output linear weights (Govindaraju and Zhang, 2000). The overall design of the RBF network is simpler than the MLP network, leaving less to experiment with in terms of determining the numbers of hidden layers and total neurons within each hidden layer. In addition, the training of the RBF network is reported to be much faster than the MLP and can handle noisy input data very well. 
However, the network also has limitations in that it typically doesn't behave well in data space that hasn't been included in the training data (Govindaraju and Zhang, 2000). The RBF network has been successfully used for hydrological applications on landscapes (Govindaraju and Zhang, 2000; Lin and Chen, 2005; Moradkhani et al., 2004).

\subsubsection{Probabilistic Neural Networks}

The Probabilistic Neural Network (PNN) is a supervised ANN model that has its roots in Bayes classifiers and is a subset in a group of neural networks called radial basis networks. PNN relies on the RBF and probability distribution function (pdf) to classify data (Nikolaev, 2008). Through the training process, the PNN learns to approximate the pdf of the training sets, which ultimately assigns a degree of membership to the defined classes. The PNN is structured using four layers consisting of the input, radial basis layer, competitive layer, and output (Demuth and Beale, 2007; Ermini et al., 2005). The basic process brings the input data in, computes the distance between the input and trained data vectors based on the RBF, sums the vectors, and presents a vector of probabilities to the competitive transfer function where the vector with the maximum probability is chosen as the winner (Demuth and Beale, 2007).

While PNNs aren't as commonly used as other supervised ANNs, the literature suggests their use in classifying the landscape has fair and promising results. Figueiredo and Gloster (1998) state that the PNN classifier "presented good accuracy, very small training time, robustness to weight changes, and negligible retraining time." A landslide hazard assessment conducted by Ermini et al. (2005) evaluated PNN models and found only results to be a fair predictor when compared to field data. Finally, in the ArcSDM application (Sawatzky, 2004), PNN is integrated as a part of the standard tool set for predicting mineral occurrence. One reported disadvantage of PNN is the large memory and computational power required to run it (Demuth and Beale, 2007).

\subsubsection{Unsupervised Artificial Neural Networks}

Unsupervised ANNs offer a large research area and show considerable promise for use in many fields. Their use is not widespread like that of supervised ANNs, yet unsupervised ANNs seem to have a future potential for providing a more 
autonomous capability in decision-making processes. Unsupervised ANNs have the ability to discover structure and natural clusters within a set of data for which no classes have been defined. Unsupervised neural networks can be beneficial in at least three basic conditions. First, in a supervised ANN, it is possible to force input data into defined classes, while an unsupervised ANN might reveal a distinction in the natural data. Second, there are situations for which no class information is available because a given situation has never occurred, has only occurred in limited space, or the data are entirely unknown. Finally, unsupervised ANNs are capable of discovering and clustering complex data relationships that can lead to determining class definitions or revealing previously unknown data relationships that weren't readily obvious.

The unsupervised ANN works to extract knowledge by exploring redundancy in the dataset, thus finding and grouping similar data patterns. This type of processing is especially effective for dealing with complex multivariate data where it may be difficult to determine data relationships and patterns. Unsupervised neural networks rely on varying network topologies and adjustment parameters such as initial neighborhoods, decay functions, and step size to control the internal learning. The most commonly used unsupervised ANN is the SOM, also referred to as a Kohonen Network, Kohonen Map, or Self-Organizing Feature Map (SOFM). The premise of this research is based upon the understanding, integration, and use of the SOM in GISc. The following section provides additional detail on SOM neural networks.

\subsubsection{Self-Organizing Maps}

The SOM is an unsupervised ANN that projects and maps high-dimensional, complex, nonlinear data to iteratively organized clusters, in a topology-preserving manner, for the creation of a low-dimensional discrete data space. The space can be used for a wide variety of purposes including speech recognition, industrial process control, image analysis, data mining, DNA sequencing, data visualization, and more (Bryan, 2006; Chon et al., 1996; Kohonen, 1982; Kohonen, 2001; Schmuker et al., 2007). In broad terms, the SOM model takes input data, establishes the nonlinear statistical relationships in a high-dimensional dataset, and transforms the data into a topology-preserving geometric structure in low-dimensional form. The SOM “...can be characterized as a two-dimensional, finite-element 'elastic surface' or network that is fitted to the distribution of the input samples" (Kohonen, 2001). The added value of the SOM is its ability to discover hidden data patterns, structures, and relationships in 
multivariate datasets. It also can conceptualize and map data in one-dimensional (1D), two-dimensional (2-D), or three-dimensional (3-D) output space using a variety of topological structures (e.g., linear, rectangular, toroidal, spherical, cubic, etc.).

The concepts of the SOM were originally proposed by Willshaw and von der Malsburg (1976), but it was Kohonen (1982) who developed the algorithms and has actively fostered their growth and capability. Because the SOM classifies data in an unsupervised form, no training data are presented to the network; thus, there is no a priori knowledge about the data distributions or the placement of data into discrete output space. In addition, no hidden layers are established in this type of network; instead, the input data and neurons are applied to a single combined input-hidden layer of weighted connections (see Figure 3.7). As mentioned previously, the SOM network establishes the topologic structure of the data. This structure is obtained by repeatedly presenting the input data signals to the network and adjusting the network weights to create "meaningful order, as if some feature coordinate system were defined over the network" (Kohonen, 2001). With SOMs, there are no assumptions about the number of classes that need to be created; in fact, some researchers suggest the use of a neuron for every attribute in the input space just to ensure a full representation of the data (Bryan, 2006; Chen, 1995). Using a high number of neurons (i.e., classes) can provide a meaningful way to initially understand relationships and patterns in the data. This approach can be followed by experimentation with a specific number of classes to identify a balance between the number of classes and proper data representation. It should be made clear that in SOMs each neuron introduced into the network attempts to establish and compete for its ownership over sets of similar data. However, this practice does not mean that every neuron has to be used. The SOM only uses the number of neurons necessary to represent the data. 


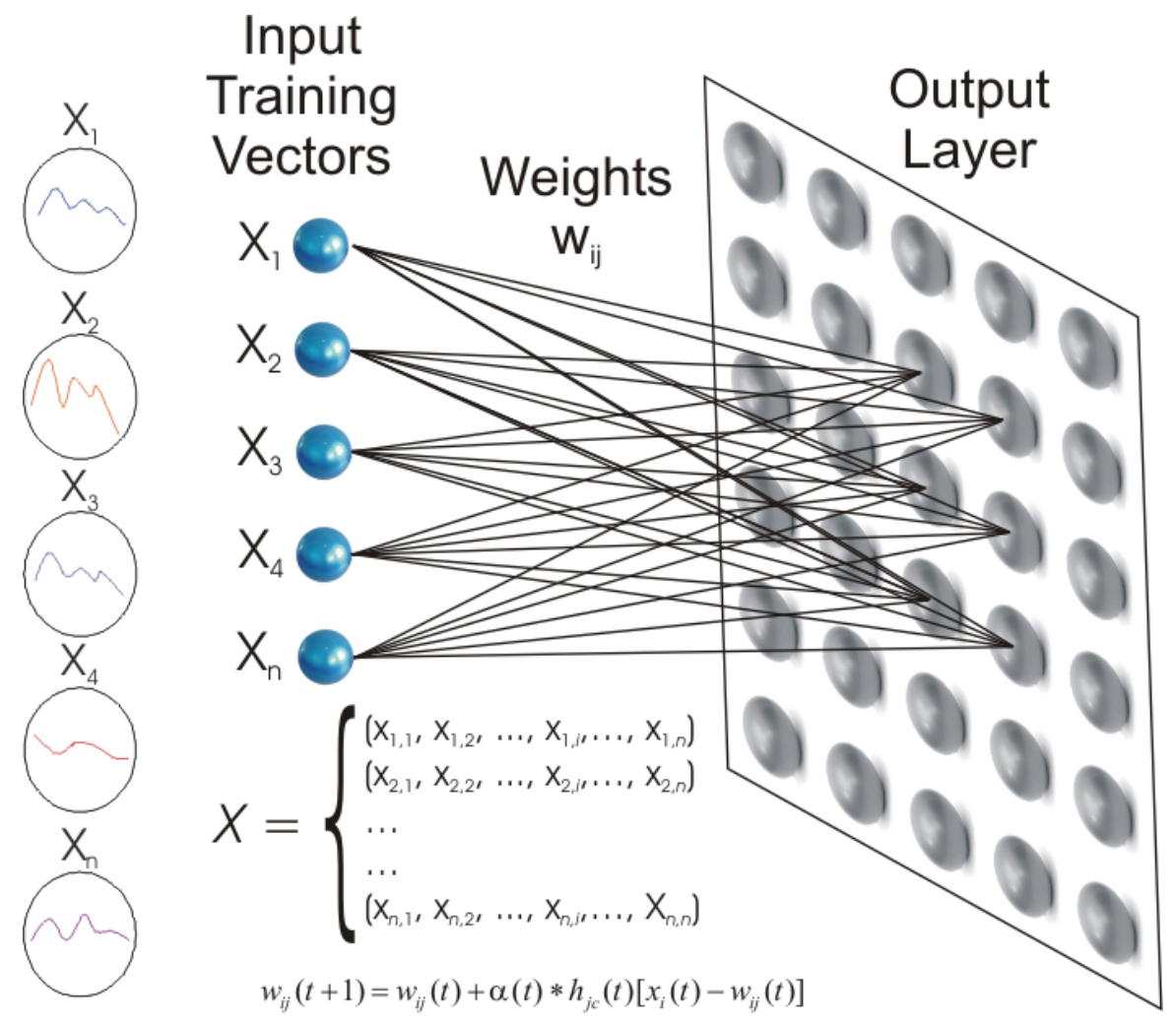

Figure 3.7. A representation of the single-layer Self-Organizing Map process as it presents data to the network, competes, and maps organized data clusters to a defined 1-D, 2-D, or 3-D topology.

\section{An Overview of the SOM Algorithm}

Self-Organizing Maps fall under the heading of "competitive soft-learning algorithms," which means that the neurons in the network compete against each other to match input data that are randomly presented to the neurons. The algorithm defined herein is summarized from the works of Kohonen (2001), Fritzke and Loos (1997), and Schmuker et al. (2007). When the SOM network is first initialized, a random set of network weights $\left(w_{i j}\right)$ is generated (see Figure 3.8), after which the competition and learning process begins with the random presentation of the input data signal or codebook vector, $(\xi)$ from matrix $(X)$, which takes the following structure:

$$
X=\left\{\begin{array}{l}
x_{1,1}, x_{1,2}, \ldots x_{1, i}, \ldots x_{1, n} \\
x_{2,1}, x_{2,2}, \ldots x_{2, i}, \ldots x_{2, n} \\
\ldots \\
\ldots \\
x_{n, 1}, x_{n, 2}, \ldots x_{n, i}, \ldots x_{n, n}
\end{array}\right\}
$$


The "winning" neuron, $\left(n_{w} \xi\right)$ is then determined by evaluating the random codebook vector that was presented to the network, (i.e., a single entry of multivariate data from input data matrix) against all network neurons in the model. A graph-based distance measure such as the $L_{1}$-norm (i.e., the Manhattan distance or the city-block metric) or Euclidean distance is calculated to determine the absolute minimum value between the input vector $(\xi)$ and the training neuron vector $(\eta)$, after which the "winning" neuron is identified and defined by $d(\eta, \xi)$.

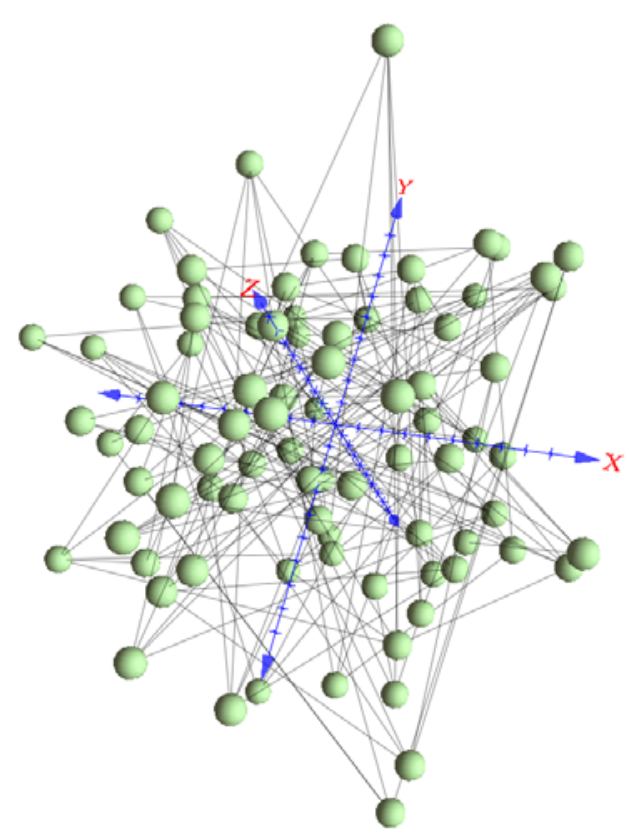

Figure 3.8. A representation of the random weight initialization that occurs in the first phase of the SOM learning process. Input codebook vectors are presented to the randomly weighted neurons and the organization and learning process begins.

Once the "winning" neuron is selected, similar neighboring neurons are pulled toward the winner to update the overall topology and network weights, thus furthering the organizational process. This process is repeated for the set number of time-steps or until an overall measure of network structure and global minimum error is obtained. At each time-step, the training neurons are updated by the following term:

$$
\Delta \eta=\lambda(t) \cdot v\left(n_{w}, \eta, t\right) \cdot(\xi-\eta)
$$

where $(t)$ is time, $(\lambda)$ is a learning rate with indices $(i)$ and $(f)$, which state the initial time-step and maximum time-step, respectively, and are defined by 


$$
\lambda(t)=\lambda_{i}\left(\frac{\lambda_{f}}{\lambda_{i}}\right)^{\frac{t}{t^{\max }}}
$$

In a standard SOM, there is a time-decaying kernel neighborhood function $(v)$, where the neurons surrounded by the winner learn together, thus causing a pulling and stretching effect on the winning neuron and its neighbors. A Gaussian function is often used for the kernel neighborhood (see Figure 3.9) and is expressed as

$$
\begin{aligned}
& v\left(n_{w}, \eta, t\right)=\exp \left(-\frac{d_{\text {topo }}\left(n_{w}, n\right)^{2}}{2 \sigma(t)^{2}}\right) \text {, with } \\
& \sigma(t)=\sigma_{i}\left(\frac{\sigma_{f}}{\sigma_{i}}\right)^{\frac{t}{t_{\max }}} \cdot
\end{aligned}
$$

where $\left(d_{\text {topo }}\right)$ defines the shortest path in graph space.

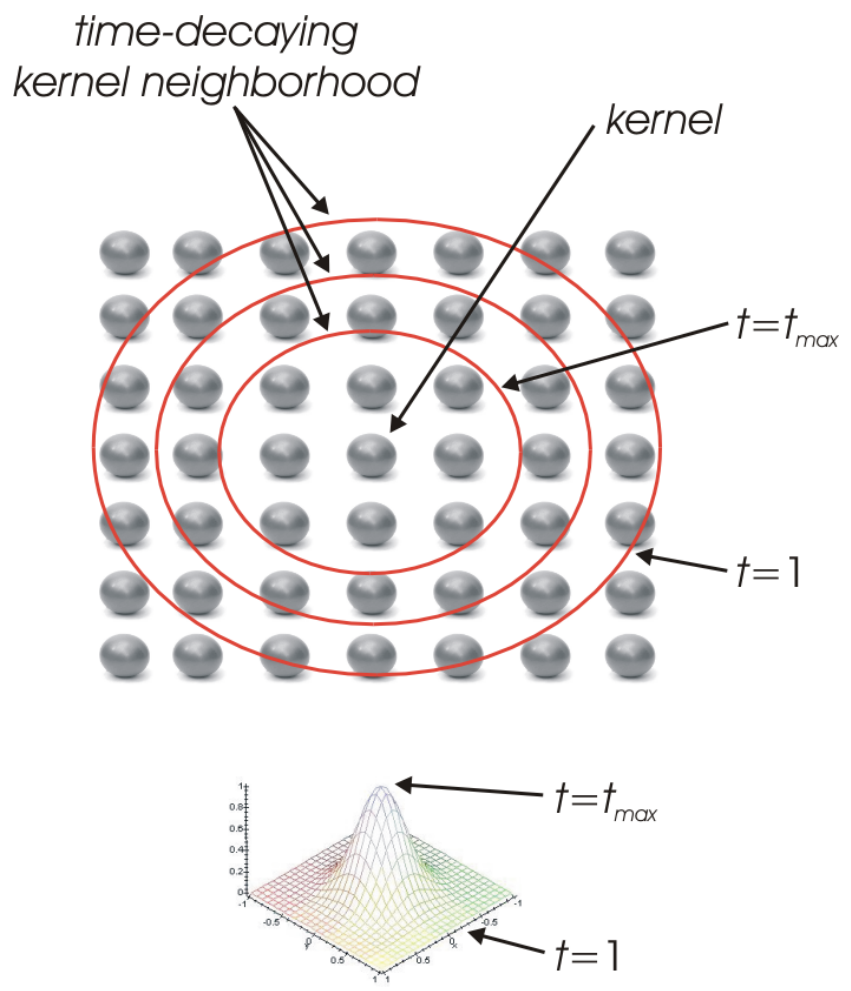

Figure 3.9. A Gaussian function is often applied to a time-decaying kernel neighborhood to update the "winning" neuron and those in the effective area. This process makes the SOM learning efficient and stable. The kernel is defined by the center point and the kernel neighborhood is represented by the red rings, which become smaller with each time-step. 
In the early learning stages, the kernel neighborhood is large. As the learning progresses, the effective neighborhood becomes smaller, more refined, and less influential-eventually maintaining a minimum neighborhood distance as the competitive learning process continues. The projection of the SOM network over the data is directly influenced by the time-decaying kernel neighborhood where the major establishment of the network occurs early in the process. Snapshots of the SOM projection over time are presented in Figure 3.10, where $\mathrm{T}=0$ are the randomly initialized neuron locations and $T=5000$ is the final result. Note that in $T=1000$, the neurons appear to be heavily grouped in one area and in general appear to be swarming in a specific direction. This is a specific example of the effect of a timedecaying kernel neighborhood where many neurons, rather than just one, are learning at the same time-step. In the case where a minimum neighborhood distance of 0 is specified, only the winning neuron is impacted. However, a minimum neighborhood of $0.05-0.5$ is common. The time-decaying kernel function has a distinct benefit of making the model come to solution more efficiently while running in a stable and safe manner (Kohonen, 2001).

The details of the SOM algorithm can be summarized as follows:

1. Initialize the weights of the training neurons $(\eta)$ to small random values.

2. Randomly select a codebook vector $(\xi)$ from data matrix ( $X$ ) for presentation to the network.

3. Calculate the distances (Euclidean or Manhattan) between the codebook $\operatorname{vector}(\xi)$ and the weights $\left(w_{i j}\right)$ of all neurons.

4. Select the "winning" neuron $\left(n_{w} \xi\right)$ from the set with the minimum distance $d(\eta, \xi)$.

5. Update the weights of the winner (Eq. 3.2 and Eq. 3.3)

6. Update the weights of the neighbors according to the distance from the "winning" neuron using a Gaussian function (Eq. 3.4)

7. Increase the time-step and repeat all steps in the algorithm until the maximum time or minimum error tolerance is reached. 


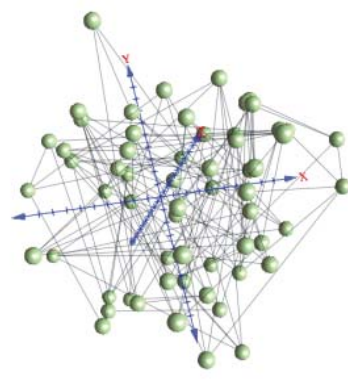

$\mathrm{T}=0$

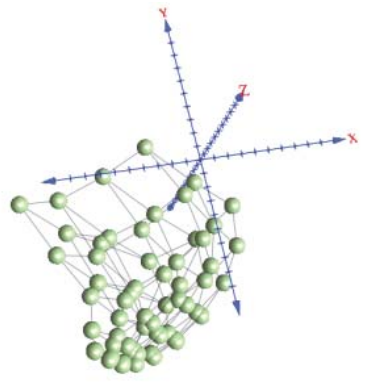

$T=1000$

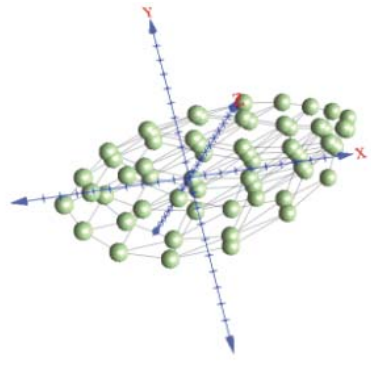

$\mathrm{T}=2000$

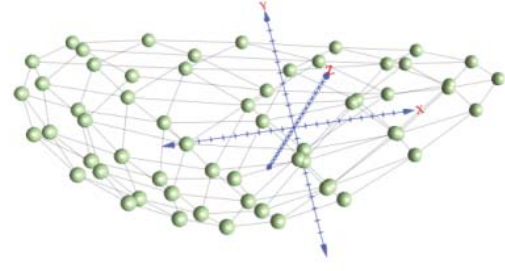

$T=3000$

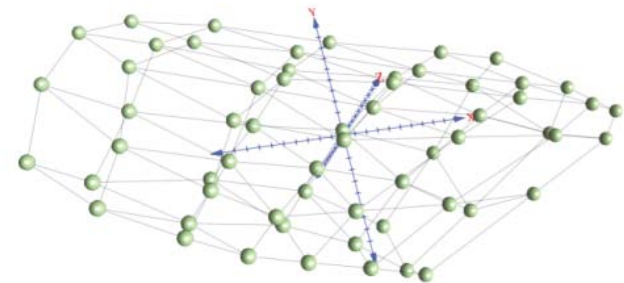

$\mathrm{T}=4000$

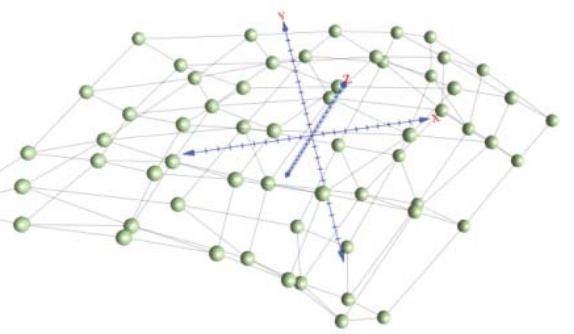

$\mathrm{T}=5000$

Figure 3.10. The SOM process captured at multiple iterations reveals the competition, learning, and projection of neurons over the input data space. Note that with an increase in iterations, the decaying kernel neighborhood function has less influence on the overall network structure and focuses on the learning and competition with individual neurons and their immediate neighbors.

\section{Demonstration of a Self-Organizing Map}

This section presents a simple demonstration to help understand the SOM and its applicability to organizing and reducing the dimensionality of complex data. The demonstration takes a complex 3-D image dataset and reduces the dimensionality while generating topological relationships. This type of demonstration is easier to understand because we are clustering colors and the result can be understood visually, thus, no further classification process is needed after the SOM training is complete.

A complex image is generated using a small script to randomly produce three values — red, green, blue (RGB) —each with a possible range of $0-255$. The result is an image of the size $450 \times 300$ pixels composed of 135,000 total values (Figure 3.11). The objective of using the SOM for this demonstration is to classify the 
complex 3-D data (i.e., red, green, and blue) and reduce the dimensionality of the original image to a size of $64 \times 64$ pixels, and then to $32 \times 32$ pixels. Within the randomly generated pixel values for the original image, there is a total of $16,777,216$ possible color values, which the SOM reduces and classifies to a possible 4,096 values (i.e., 64x64), then again to a possible 1,024 values (i.e., 32x32).

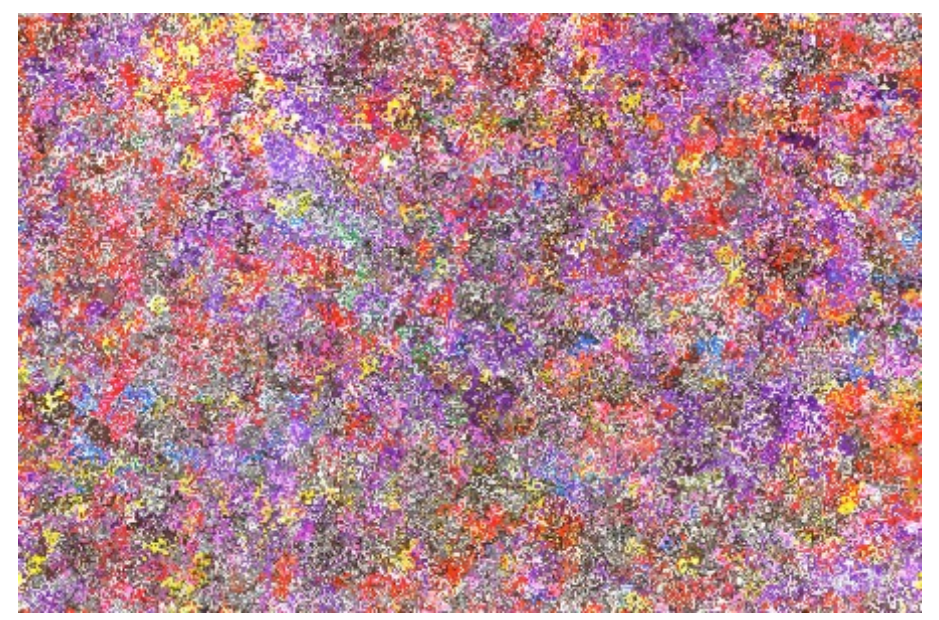

Figure 3.11. A randomly generated 450x300 RGB image with 135,000 values used as input to a SOM training process.

To begin, the 2-D SOM array is initialized with random weights (see Figure 3.8 and Figure 3.12), after which the competitive learning process begins to structure and project the data in a way that is similar to what is presented in Figure 3.10.

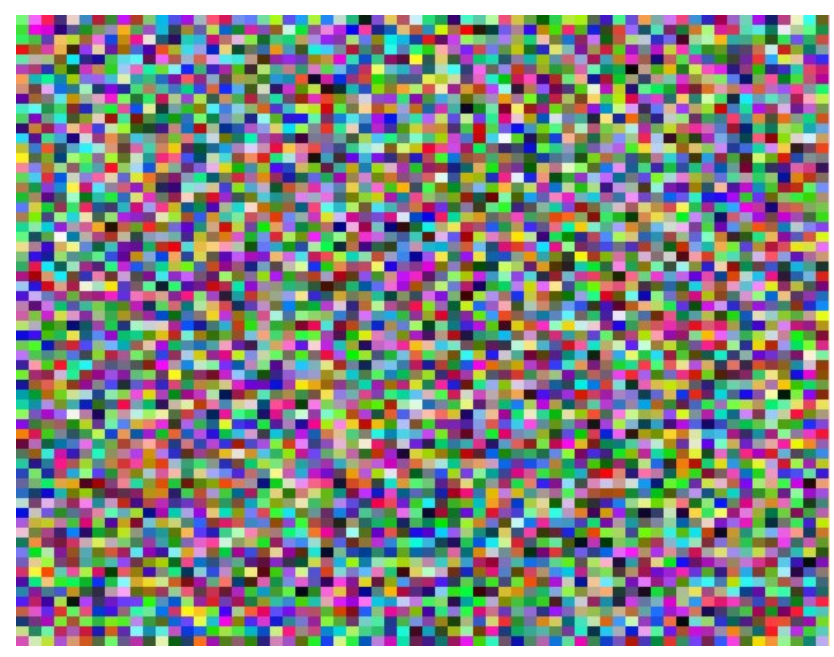

Figure 3.12. The random initialization of neuron weights in a 2-D grid is presented. Each pixel in the grid is representative of a single neuron.

The final grid structure of the SOM training is presented in Figure 3.13. For comparison, a 32×32 2-D SOM also was trained with the same input data as the 
64x64 SOM and these results are presented in Figure 3.14. Keep in mind that because the SOMs are initialized with random weights at the beginning of each training, the topological order and projection of the data vary in their location (i.e., the clusters of red-like colors currently show up in the lower-middle portion of the $64 \times 64$ grid and on the next run, these may show up in the upper left-hand corner). However, the underlying assignment and clustering of data remain nearly or exactly the same.

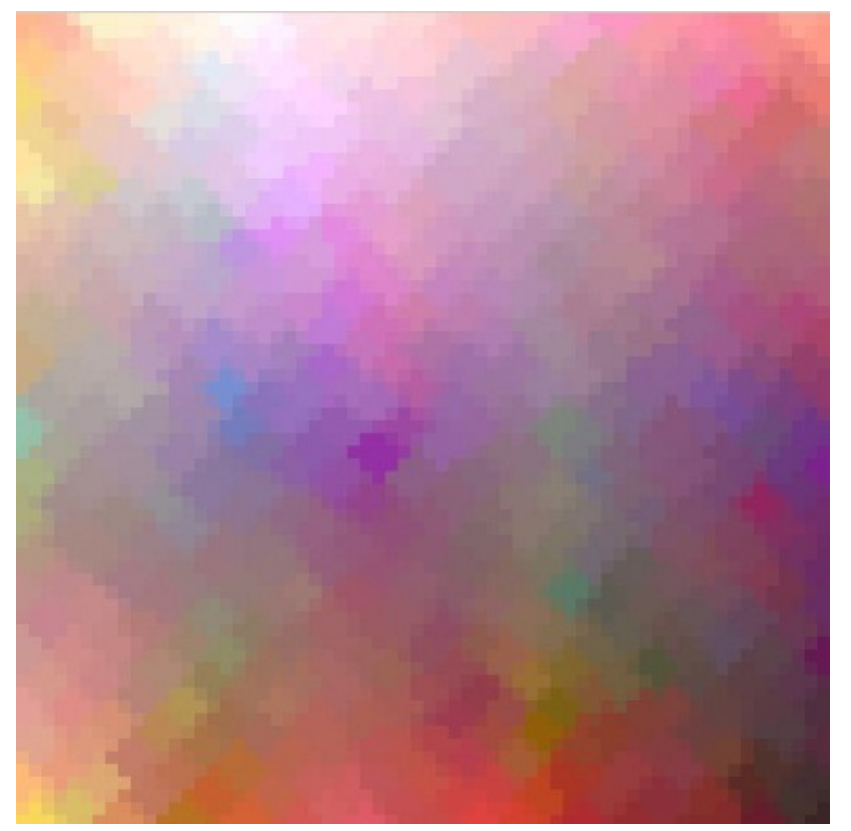

Figure 3.13. The final result of the SOM training from a 450x300 dataset reduced and organized into a $64 \times 64$ grid. 


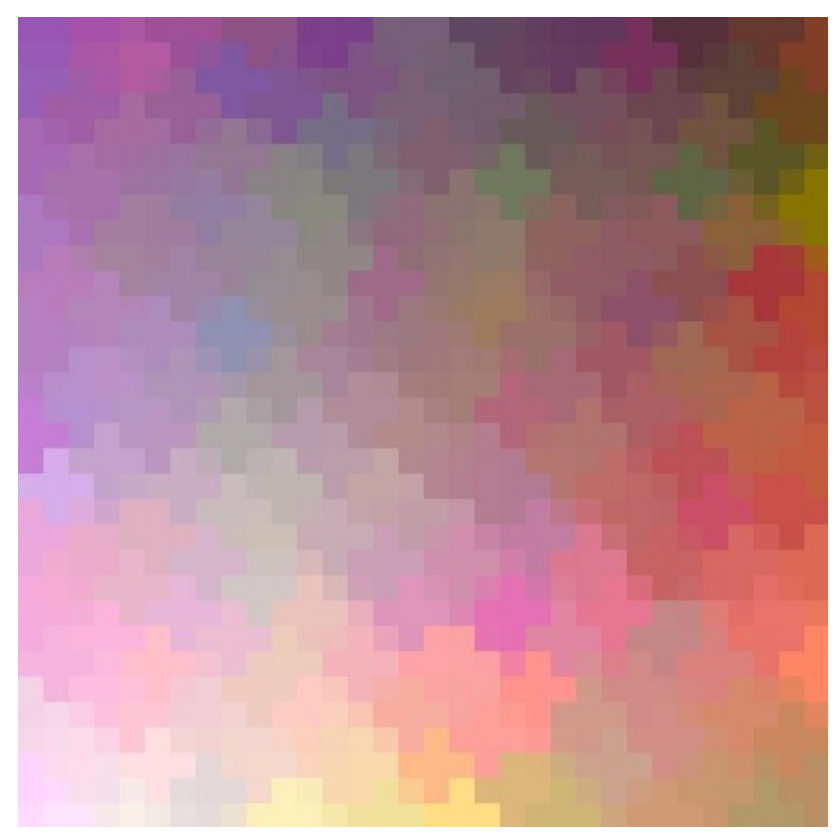

Figure 3.14. A 32x32 2-D trained SOM using the randomly generated source data presented in Figure 3.11 . 


\subsection{The Adaptive Landscape Classification Procedure}

This chapter explores a procedure (i.e., the ALCP) developed to harvest large amounts of landscape-based data, both spatial and spatio-temporal, into discrete and homogeneous classes by using the powerful capabilities of GIS and SOMs. A discussion ensues on the purpose and background for developing the ALCP and further discusses the specific components and operating mechanics including the data types that can be used, currently supported data themes, the GIS/SOM software linkage, and visualization and analysis of the classification results.

\subsection{Purpose and Background}

It is well recognized that landscapes evolve over time through a process called "ecological succession" (Cowles, 1911; Gleason, 1927). This evolution is spawned by an array of complex interrelations of spatially distributed variables, including geology, soils, precipitation, temperature, solar radiation, terrain, fluvial processes, crustal movement, mass-wasting events, chemical and physical weathering, fire events, meteorological extreme events, climate change, and varying anthropogenic influences. Because of this understanding of landscape evolution and its many possible variables, it is necessary to consider adaptability in producing discrete and homogeneous measures of the landscape and in providing a method that is adaptive to a wide range of different landscape, process, and variable types. For example, the ALCP can be used to gain an overall perspective of the landscape, incorporating all variables that are defined to take a part in the landscape, or evaluate specific phenomena in the landscape such as probable sites of mass-wasting, measures of habitat quality, hydrologic response, groundwater recharge zones, snow accumulation properties, etc. The newly introduced ALCP is designed to be generic enough to mix the variables of interest, develop a pattern of these variables, and then evaluate the patterns for similarity across the landscape. Figure 4.1 provides an example of four single-variable data patterns found in 10 separate sub-watershed catchment areas. These individual patterns are captured and combined to create a single pattern of multiple variables that is then used for the similarity analysis. As stated by Bathgate and Duram (2003), "Although the landscape is a continuum, it can be classified into discrete categories...." The process of using multiple metrics on the landscape can be likened to the idea of multi-spectral analysis in remote sensing (Brown, 1998). 

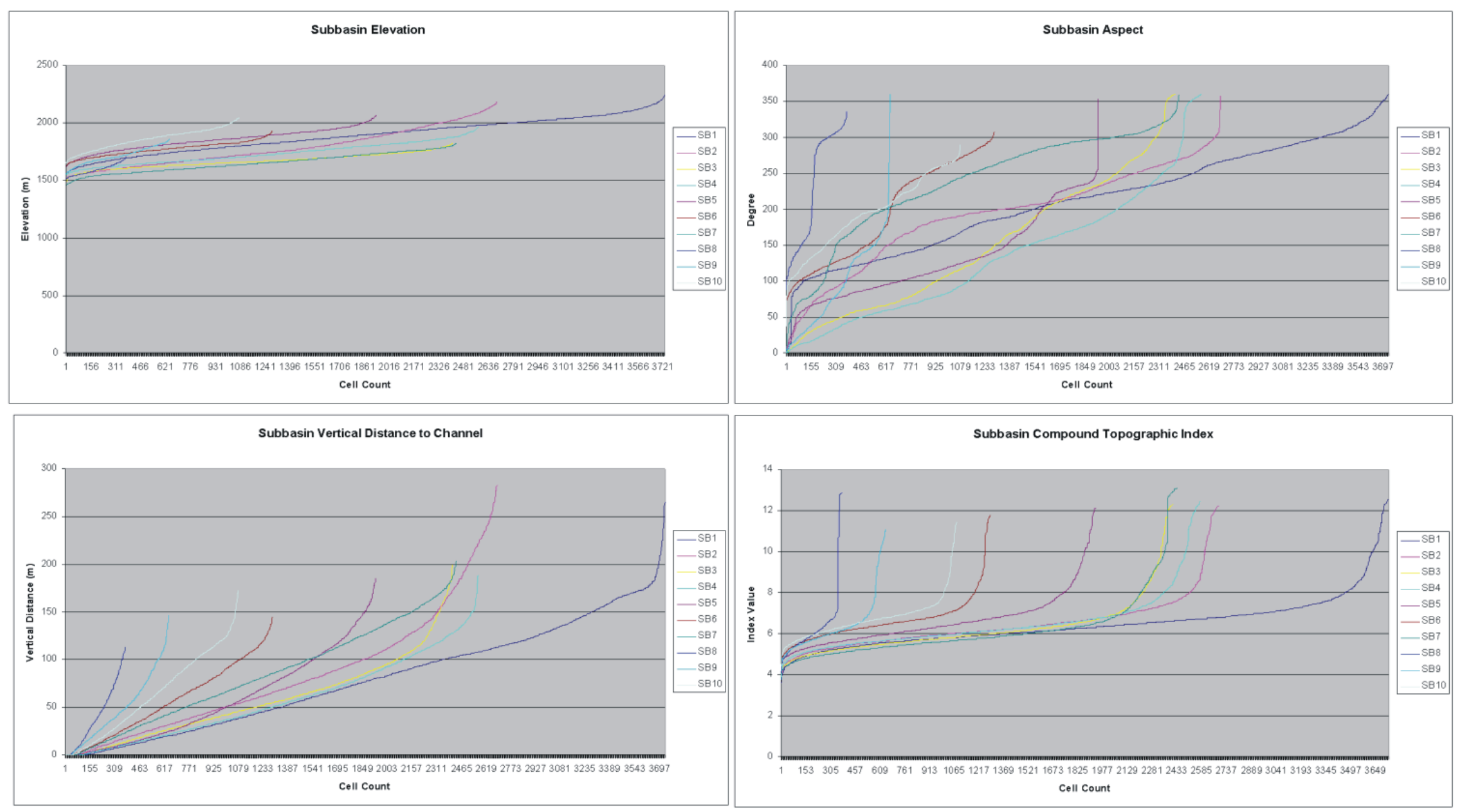

Figure 4.1. Four variables for 10 sub-basin areas are presented to illustrate different data patterns in the landscape. 
A core component of the ALCP is based on using pattern-recognition technologies developed in the machine learning and evolutionary computing sciences. The computational algorithms available to perform pattern recognition have been dramatically enhanced by rapid developments in evolutionary computing over the past few decades. Evolutionary computing methods, such as ANNs, SVMs, genetic algorithms, swarm intelligence, simulated annealing, and fuzzy logic, have made voice recognition, character recognition, data mining and/or search engines, medical diagnosis, stock market analysis, and computer gaming and/or simulators common features in modern society, and perhaps even without our recognition of what is driving these processes. These new capabilities significantly augment the statistical pattern recognition algorithms that were the basis of earlier pattern recognition methods.

It should be noted that while the focus of the study reported here is on the broader landscape scale, there is nothing about this procedure (as long as the required data are available) that would limit the ability to conduct similarity analysis on a fine scale. For example, a 500-m reach of river could be evaluated for its aquatic habitat, which might include variables such as river bathymetry and terrain-based derivatives, water flow, flow velocities, current directions, substrate, nutrient availability, riparian vegetation, aquatic vegetation, woody debris, etc. Similarly, the procedure can be used for broader-scale analysis on a regional, continental, or global basis.

\subsection{The Components and Structure of the ALCP}

The ALCP relies on a hybrid mixture of basic and advanced geospatial processing and analysis; a spatially enabled database for data input, storage, and queries; a statistical processor; a Self-Organizing Map (SOM) model to analyze and cluster input signals; and an external wrapper module to tie the components together. An overview of the components and procedural flow of the ALCP is provided in Figure 4.2. The following four major components of the ALCP are discussed in the ensuing sections:

- Source Data Elements

- Geospatial Processing

- SOM Model and Post-Processor

- Visualization and Analysis. 


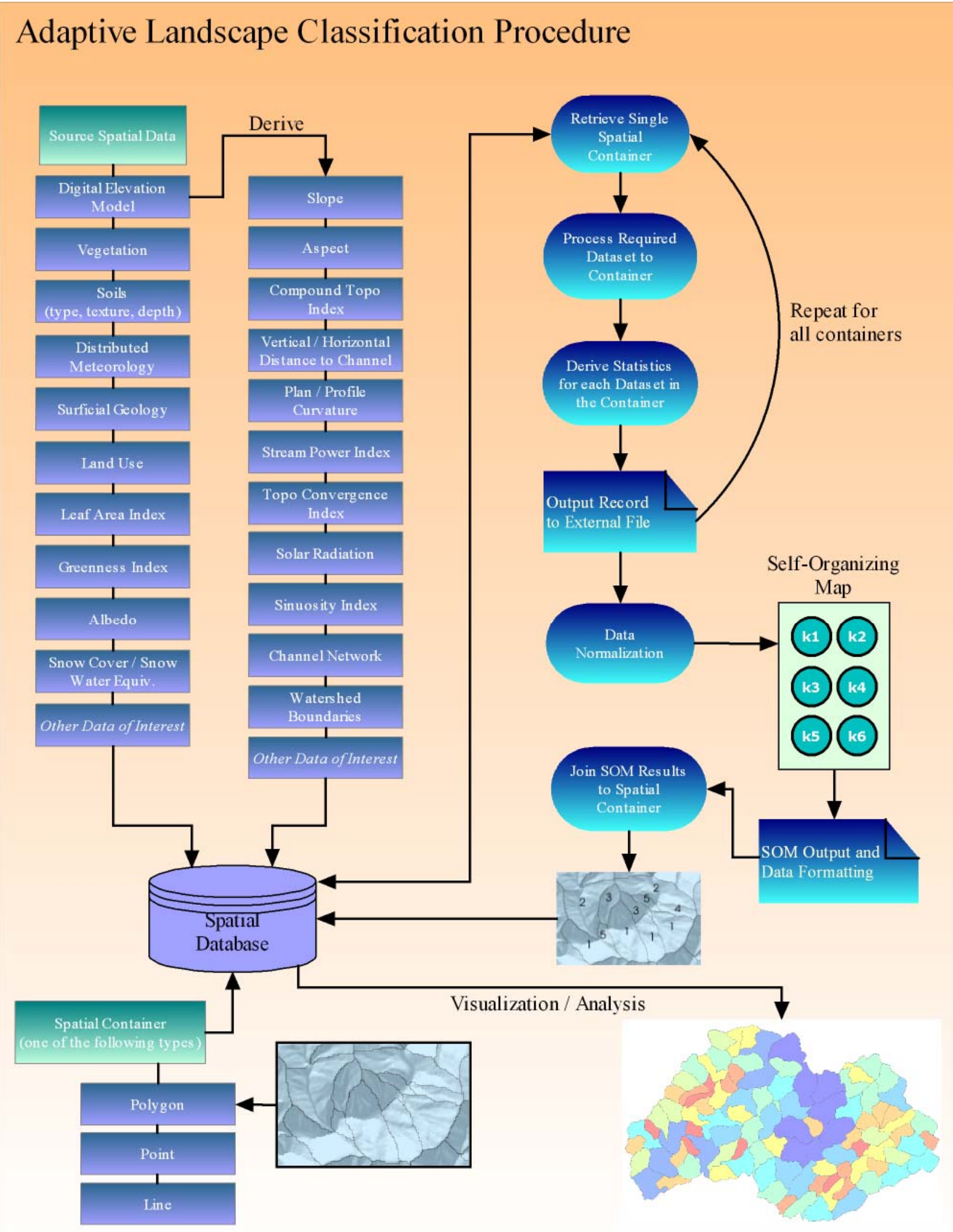

Figure 4.2. An overview of the structure and flow of the ALCP. 


\subsection{Source Data Elements}

Much of the ALCP revolves around the source data used to conduct the classification process. Figure 4.2 shows most of the datasets collected and derived for use and testing in the study. While these data cover most of what would typically be needed for landscape-level analysis, the choice of data required for analysis fundamentally depends on the problem being addressed. For example, a multivariate ecoregion study by Hargrove and Luxmoore (1998) considered detailed soils data including the nitrogen content, soil-water capacity, and organic content of the soil as well as other climate-related variables. An early design of the ALCP established a need to incorporate a range of data types, thereby providing adaptability in the data inputs. As such, the data elements for this study can be categorized into four primary categories: 1) continuous, 2) categorical, 3) discrete, and 4) spatio-temporal, all of which are described in the ensuing sections. The data and methods implemented in the ALCP for possible use in landscape classification are summarized in Table 4.1.

\subsubsection{Continuous Data}

According to Worboys and Duckham (2004) continuous "if small changes in location lead to small changes in the corresponding attribute value..." for a dataset. Continuous data are commonly found in the form of raster, or pixel-based, data such as elevation, temperature, soil moisture, and imagery and/or remote-sensing data. The data plotted in Figure 4.1 and Figure 4.3 are representative of continuous data of elevation and elevation data derivatives that are characterized by a smooth and continuous transition from one point to the next. This type of data is by far the most commonly used in the ALCP, largely due to the strong basis of primary and secondary terrain-based data and remote sensing derived datasets. The ALCP evaluates each continuous dataset and derives descriptive statistical measures for each "spatial container" (discussed in Section 4.4.1) for presentation to the SOM model. 


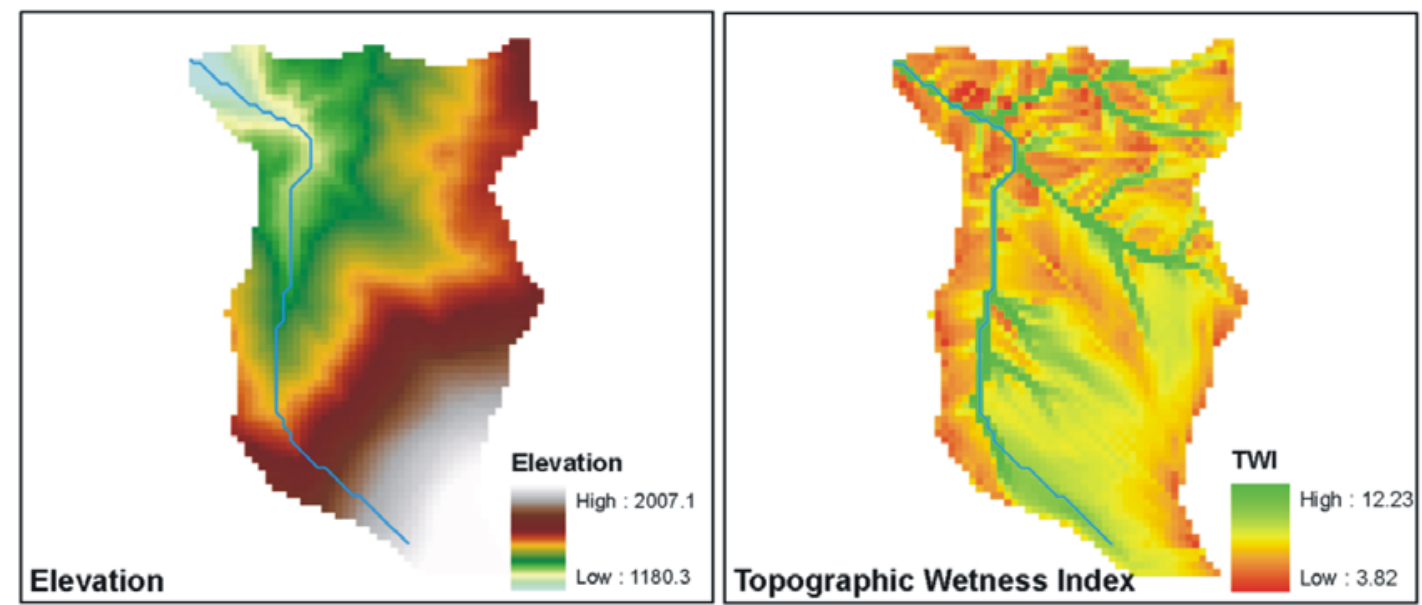

Figure 4.3. Two examples of continuous datasets that are raster-based and characterized by smooth transitions between the attributes.

Table 4.1. Terrain-based data processed and extracted within the ALCP.

\begin{tabular}{|c|c|}
\hline Data & Reference Method \\
\hline \multicolumn{2}{|c|}{ Continuous Data } \\
\hline Pit-Filled DEM & (Planchon and Darboux, 2001) \\
\hline Slope & $\begin{array}{c}\text { Fit 2-Degree Polynomial } \\
\text { (Zevenbergen and Thorne, 1987) }\end{array}$ \\
\hline Aspect & $\begin{array}{c}\text { Fit 2-Degree Polynomial } \\
\text { (Zevenbergen and Thorne, 1987) }\end{array}$ \\
\hline Curvature & $\begin{array}{c}\text { Fit 2-Degree Polynomial } \\
\text { (Zevenbergen and Thorne, 1987) }\end{array}$ \\
\hline Plan Curvature & $\begin{array}{c}\text { Fit 2-Degree Polynomial } \\
\text { (Zevenbergen and Thorne, 1987) }\end{array}$ \\
\hline Profile Curvature & $\begin{array}{c}\text { Fit 2-Degree Polynomial } \\
\text { (Zevenbergen and Thorne, 1987) }\end{array}$ \\
\hline Convergence Index & (Koethe and Lehmeier, 1996) \\
\hline Solar Radiation & (Wilson and Gallant, 2000) \\
\hline Flow Direction \& Accumulation & (Tarboton, 1997) \\
\hline Flow Sinuosity & (Olaya, 2004) \\
\hline Overland Flow Distance to Channel & (Conrad and Ringeler, 2007) \\
\hline Vertical Distance to Channel & (Conrad and Ringeler, 2007) \\
\hline Topographic Wetness Index & (Moore et al., 1991) \\
\hline Stream Power Index & (Moore et al., 1991) \\
\hline Length-Slope Factor & (Moore et al., 1991) \\
\hline Topographic Roughness Index & (Riley et al., 1999) \\
\hline Soil Depth & Multi-Layer Soil Characteristic Database \\
\hline
\end{tabular}




\begin{tabular}{|c|c|}
\hline & (Miller and White, 1998) \\
\hline Soil pH & $\begin{array}{l}\text { Multi-Layer Soil Characteristic Database } \\
\text { (Miller and White, 1998) }\end{array}$ \\
\hline Soil Water Capacity & $\begin{array}{c}\text { Multi-Layer Soil Characteristic Database } \\
\text { (Miller and White, 1998) }\end{array}$ \\
\hline Soil K-factor (particle transport) & $\begin{array}{l}\text { Multi-Layer Soil Characteristic Database } \\
\text { (Miller and White, 1998) }\end{array}$ \\
\hline \multicolumn{2}{|c|}{ Categorical Data } \\
\hline Landform Class & (Peucker and Douglas, 1975) \\
\hline Land Cover & $\begin{array}{c}\text { Multi-Resolution National Land Cover } \\
\text { Database (NLCD) (Homer et al., 2004) } \\
\& \\
\text { Global Land Cover Characterization Program } \\
\text { (GLCP) (Loveland and Belward, 1997) }\end{array}$ \\
\hline \multicolumn{2}{|c|}{ Discrete Data } \\
\hline Contributing Area & (Tarboton, 1997) \\
\hline Channel Network & (Tarboton, 1997) \\
\hline Channel Gradient & ALCP-Generated \\
\hline Channel Aspect & ALCP-Generated \\
\hline Catchment Area & ALCP-Generated \\
\hline Catchment Perimeter & ALCP-Generated \\
\hline Catchment Elongation Ratio & ALCP-Generated \\
\hline Channel Density & ALCP-Generated \\
\hline Longest Channel Length & ALCP-Generated \\
\hline Mean Catchment Slope / Aspect & ALCP-Generated \\
\hline \multicolumn{2}{|c|}{ Spatio-Temporal } \\
\hline Soil Moisture & $\begin{array}{l}\text { Remotely-Sensed Microwave Radiometer } \\
\text { (Njoku, 2007) }\end{array}$ \\
\hline Normalized Difference Vegetation Index & $\begin{array}{l}\text { Normalized Difference Vegetation Index } \\
\text { (Carroll et al., 2004) }\end{array}$ \\
\hline Albedo & $\begin{array}{l}\text { Filled Land Surface Albedo } \\
\text { (NASA, 2007) }\end{array}$ \\
\hline Distributed Meteorology & $\begin{array}{c}\text { Parameter-elevation Regressions on } \\
\text { Independent Slopes Model (PRISM) } \\
\text { (Daly et al., 1994) }\end{array}$ \\
\hline Snow Water Equivalent & $\begin{array}{c}\text { Snow Data Assimilation Model } \\
\text { (NOHRSC, 2007) }\end{array}$ \\
\hline Meteorology Forecast & $\begin{array}{l}\text { U.S. National Weather Service, National } \\
\text { Digital Forecast Database (NDFD) }\end{array}$ \\
\hline
\end{tabular}




\begin{tabular}{|c|c|}
\hline & (NWS, 2007) \\
\hline Solar Radiation & (Wilson and Gallant, 2000) \\
\hline
\end{tabular}

Early and significant research for this study was conducted on extracting primary and secondary topographic data to realize and produce important elements in landscape processes. The landscape terrain is a fundamental element that has a direct effect on a variety of processes ranging from solar irradiance to soil moisture to slope stability. The Digital Elevation Model (DEM) provides a significant and rich resource for landscape classification and is a significant source of data made available in the ALCP.

Terrain data (i.e., DEMs) are available throughout the world making this a valuable base resource for which to conduct landscape classification. The Shuttle Radar Topography Mission (SRTM) data is available near globally (+/- $60^{\circ}$ latitude) at a reasonably high resolution (i.e., 1 arc-second; 3 arc-second) and are free of cost. Additionally, higher spatial resolution of the SRTM data are also available for certain areas of the world or can be made available by special request. Other terrain data is available through many individual government and private sources produced using varying methods (e.g., photogrammetric extraction, Light Detection and Ranging [LiDAR], land survey, etc.). Many studies have connected terrain-based attributes to elements such as soil depth, soil moisture, surface and sub-surface hydrologic response, sediment transport, landslide probabilities, vegetation distribution, ecological response, and more (Burt and Butcher, 1985; Catani et al., 2005; Dubayah and Rich, 1995; Matthes-Sears et al., 1988; Moore et al., 1991; Nellemann and Fry, 1995; Shaver et al., 1990; Tarboton, 1997; Wilson and Gallant, 2000).

Terrain analysis is heavily used in the ALCP to determine numerous hydrological, geomorphological, and ecological parameters that help to define and describe complex landscape processes and phenomena. Much of this analysis involves evaluating spatial patterns in the terrain to derive elements such as landform characteristics, stream channel sinuosity, incoming solar radiation, soil moisture, and more. Terrain-based data derivatives can be classified into primary and secondary attributes. Primary attributes are those that can be evaluated directly from the DEM data, such as slope, aspect, and plan and profile curvature. Secondary attributes typically take a primary topographic dataset along with one or more additional datasets and sometimes a simplified model to produce additional datasets (Wilson 
and Gallant, 2000). Examples of secondary topographic attributes include incoming solar radiation, soil moisture, erosion indices, topographic wetness index, and overland flow distance to a channel.

An important driver in landscape characterization is rooted in hydrologic processes including the patterns and behaviors of precipitation, streamflow, and groundwater recharge. For this research study, considerable effort has been put forth to develop routines within the ALCP that are capable of extracting hydrologic parameters which can then be used in the classification process. The methods by which hydrologic parameters are derived may differ from the commonly used D8 algorithm found in most GIS software packages (Costa-Cabral and Burges, 1994). Using the DEMON (digital elevation model network extraction) algorithm (Costa-Cabral and Burges, 1994) or the $D^{\infty}$ (deterministic infinity) (Tarboton, 1997) algorithm, better quality and more realistic hydrological parameters can be estimated. Terrain-extracted data also can be used with other raster- and vector-based data to derive critical landscape characteristics such as road and/or stream crossing densities, length of roads in close proximity to streams, road densities, streams adjacent to various land use and/or land cover types, upslope and downslope population densities, riparian and wetland areas, climate, soil types and properties, existence of dams, and more.

It should be noted that for this discussion, a general distinction exists between "static" types of continuous data, which would include elements with longer-term fixed properties such as terrain, and "spatio-temporal" data, which are continuous data with temporally frequent changes (i.e., daily to monthly time-scale) such as meteorology and soil moisture.

\subsubsection{Categorical Data}

Data that have already been lumped into a classification field are commonly referred to as categorical data. This type of data is typically continuous over space and data attributes are presented as alphanumeric descriptions or numerical values. In the case of numerical attributes used to describe categorical data, these are in fact data abstractions, as opposed to a real measured value, used to represent a descriptive attribute such as a land use or soil type. The ALCP evaluates the total number of unique categories in the area of interest and establishes global fields in the data presented to the SOM model. When each spatial container is evaluated, either an 
area percentage or actual area value typically is calculated and inserted into the global fields. For example, if a study area exhibits 10 classes of vegetation, a global numeric field is established for each class. Then when a single spatial container is evaluated and only 3 of the 10 classes exist in this area, the SOM input file is populated with the values for these three classes and the remaining seven fields are set to zero. An example of two categorical datasets is shown in Figure 4.4 and datasets that can be used in the ALCP are presented in Table 4.1. Note that although vegetation and land-use characteristics are dynamic and have the potential to change over time, these data are not included in the spatio-temporal data category (discussed in the following sections) because they need to be distinguished by their thematic type attributes.

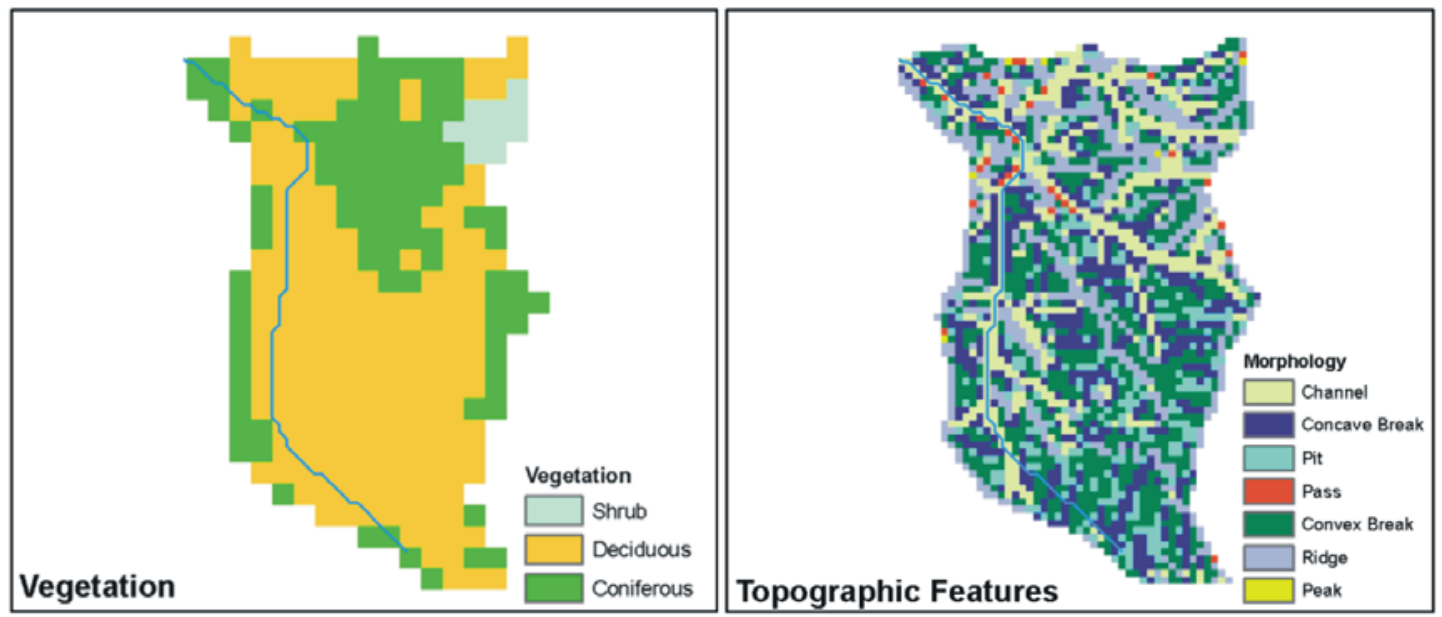

Figure 4.4. Categorical data comprise a dataset classified by pre-defined groupings as presented in the two examples.

\subsubsection{Discrete Data}

In a geographical context, a discrete data type can be defined as data that represent a distinct phenomenon, characteristic, or attribute, and are contained within an established boundary. Examples of this type of data include sub-basin area and perimeter; density measurements such as population, streams, or roads; longest channel length; mean channel slope and aspect; basin shape index; or other boundary-encompassing index values. The building blocks for the landscape classification is a spatial container, so anything that can describe this area with a single value can be used. Discrete data generated by the ALCP are provided in Table 4.1. 


\subsubsection{Spatio-Temporal Data}

Spatial variations that occur within a fixed position in space at a defined time-interval can be described as spatio-temporal. The notion of spatio-temporal information systems has been coming into reality as technology has adapted to collect and distribute data in real-time or near real-time, especially in a spatial construct such as is being developed in the Sensor Web protocols (OGC, 2007). As discussed in the introduction to this chapter, we understand landscapes to evolve over time (natural and anthropogenically influenced) and there is a need to learn and adapt to a flow of data that has the potential to change over time at varying rates and perhaps even in a cyclic form. With such a flow of data constituting large quantities of information, establishing the presence or absence of spatio-temporal patterns becomes more difficult. It is conceivable that in situ measurements can be used more effectively and distributed to a wider area as relationships between ground-based observational data and remotely sensed spatio-temporal data are established. In the context of hydrological modeling and characterization, an understanding of spatially distributed meteorology conditions, snowpack conditions (where and when appropriate), and soil moisture over a regular interval of time can provide a capability for increased forecasting methods to evaluate water supplies, energy management, flood warnings, and environmental regulation. The spatio-temporal component of the ALCP is an important factor for bringing classification methods to a real-time, near real-time, or historically reconstructed basis. Examples of spatio-temporal datasets are presented in Figure 4.5 and Figure 4.6 and data processed and extracted by the ALCP are listed in Table 4.1. 


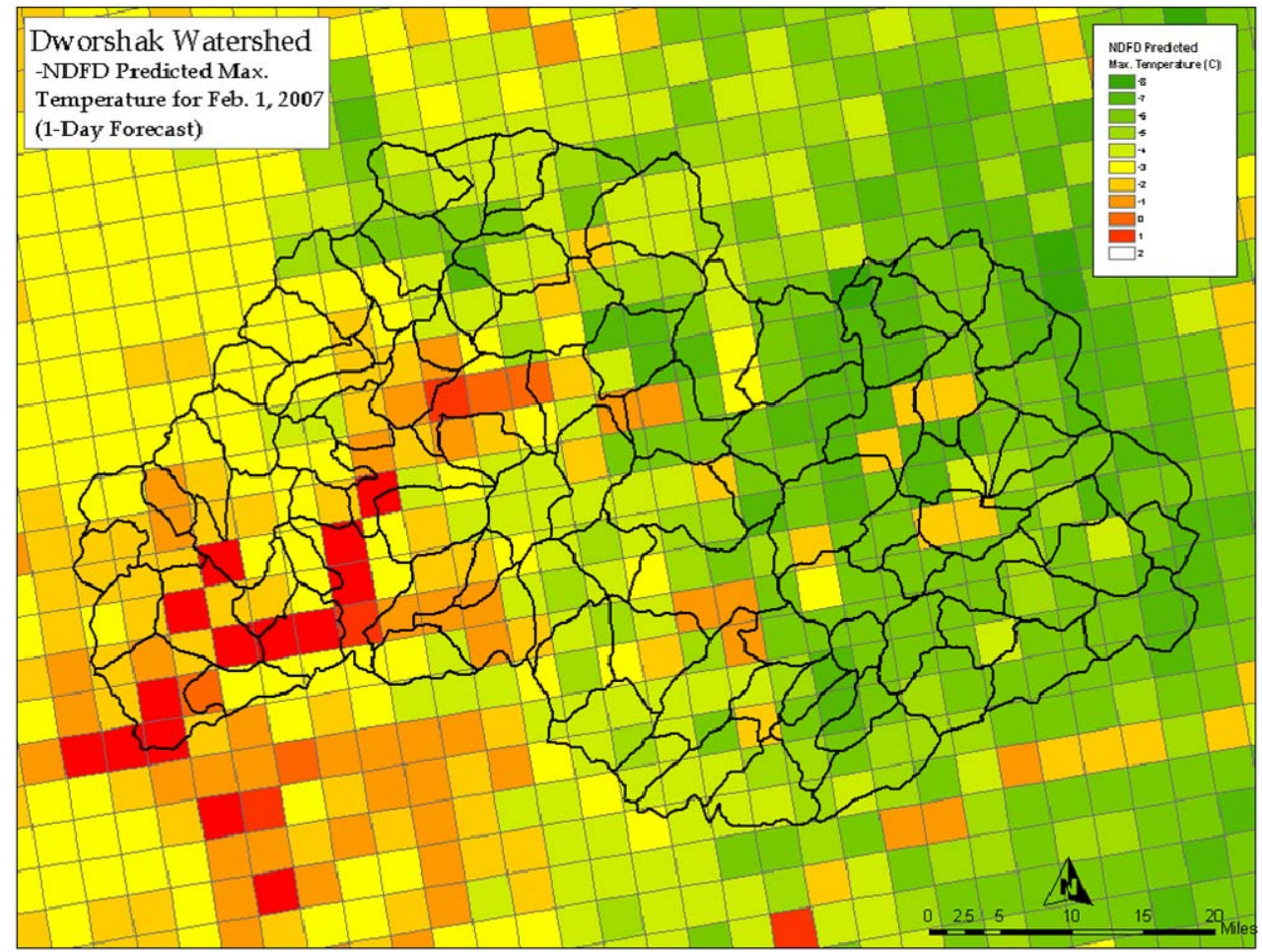

Figure 4.5. Spatio-temporal data representing a 1-day maximum temperature forecast condition from the National Digital Forecast Database (NDFD) meteorology model (NWS, 2007).

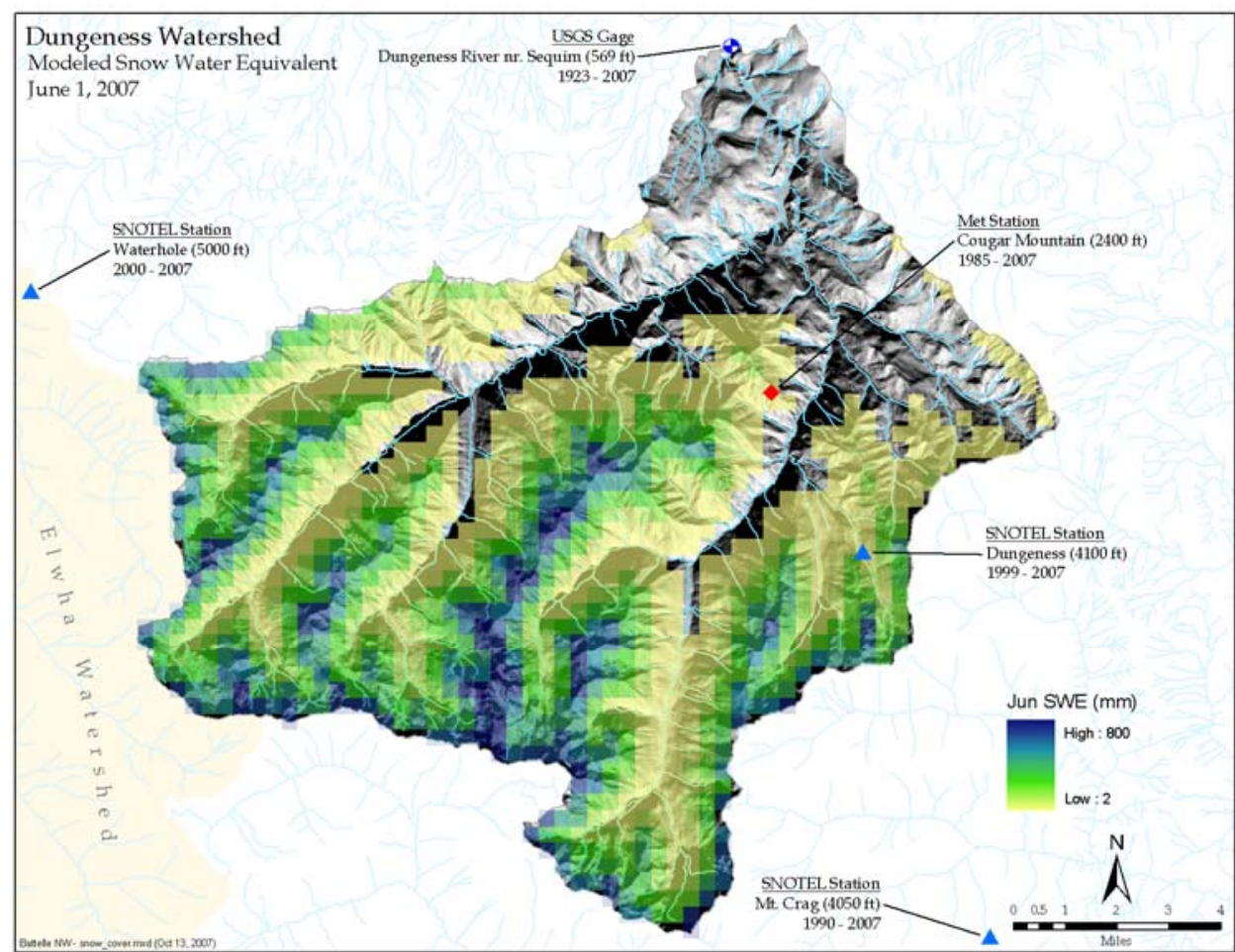

Figure 4.6. A spatio-temporal dataset representing snow-water equivalent conditions for a given day. New data results are produced daily from the United States National Oceanic and Atmospheric Administration's Snow Data Assimilation Model (SNODAS) (NOHRSC, 2007). 


\subsection{Geospatial Processing}

Much of the ALCP involves the feeding and geo-processing of the source datasets, and in the case of the terrain data, derivative datasets are generated and made available for the classification process. A series of scripts were developed (see Appendix $A$ ), using the Arc Macro Language (AML) to interface with functions in the Arc/INFO GIS software and Python programming language to interface with the ArcGIS and the open-source System for Automated Geoscientific Analysis (SAGA) GIS software (Conrad and Ringeler, 2007; ESRI, 2007). These scripts provide a number of functions relating to storage on and retrieval from the spatial data base; performing coordinate projections; clipping and buffering data; extracting terrainbased derivative data; determining discrete data attributes such as stream lengths, stream gradients, watershed areas, etc.; processing and controlling the spatial containers; deriving statistics of container data; and preparing data for the SOM processing. Three fundamental processing elements occur in the geospatial processing stage of the ALCP. The first was largely addressed in Section 4.3 as it relates to source data and the methods of generation used for these datasets. It should be made clear that during the first stage of gathering and producing data, a master database for the area of interest is populated and made ready for the remaining two processes, which prepare information for the SOM model. These two processing elements are discussed here.

\subsubsection{Spatial Container}

Issues of spatial scale and resolution are common topic and research areas in the field of geography and many other disciplines that use this type of data. While there are many facets of the issues surrounding scale, the basic concept relates to the level of generalization applied as an interpretation of reality. It is clear from the vast amount of literature written on the topic of scale over the past three decades that this is a complex issue. This study did not attempt to solve these complex scaling issues; rather it provided a path for bringing various data types and scales into a common entity, so that they can be presented to the SOM model for pattern analysis. Scale issues must be considered when presenting data into the ALCP. For example, a 25$\mathrm{km}$ pixel-resolution remote-sensing dataset would not be appropriate for a small watershed measuring $500 \mathrm{~km}^{2}$ in area (i.e., would only include a single pixel), but rather more suitable for regional, continental, and global scales. The open structure 
of the ALCP is intended to be used at varying scales from the small local watershed to a continental or global scale if desired.

The "spatial container" is a method for which multiple datasets of varying scales and data types can be gathered together under a specific polygon, point, or line domain, and information about the data can be harvested and organized in a common way using a common spatial database. An example of a spatial container dataset is presented in Figure 4.7, where small sub-watershed boundaries were generated to classify the landscape at a fine level of detail. Spatial containers can be used to represent any kind of spatial domain that is suitable for investigating the data and area of concern. For example, a spatial container can be defined by vegetation, land use, or population density boundaries; random point samples; in situ observation points; or linear transects representing migratory routes or other meaningful linear entities. The only requirement for the spatial containers as a whole is that enough containers with variability in their attributes are provided so that spatial patterns can become meaningful after the SOM model returns the results to the GIS. The basic process for using spatial containers is to 1) use a single container to harvest and organize information from the required datasets, 2) store the data into a spatial database using a unique spatial container identifier, 3) repeat steps 1 and 2 for all containers, 4) dump the results of all containers for input to the SOM model, 5) run the SOM model, 6) process results back to the GIS, and 7) view and analyze the classified spatial container. A simple representation of this process is shown in Figure 4.8 and the overall process is shown in Figure 4.2. 


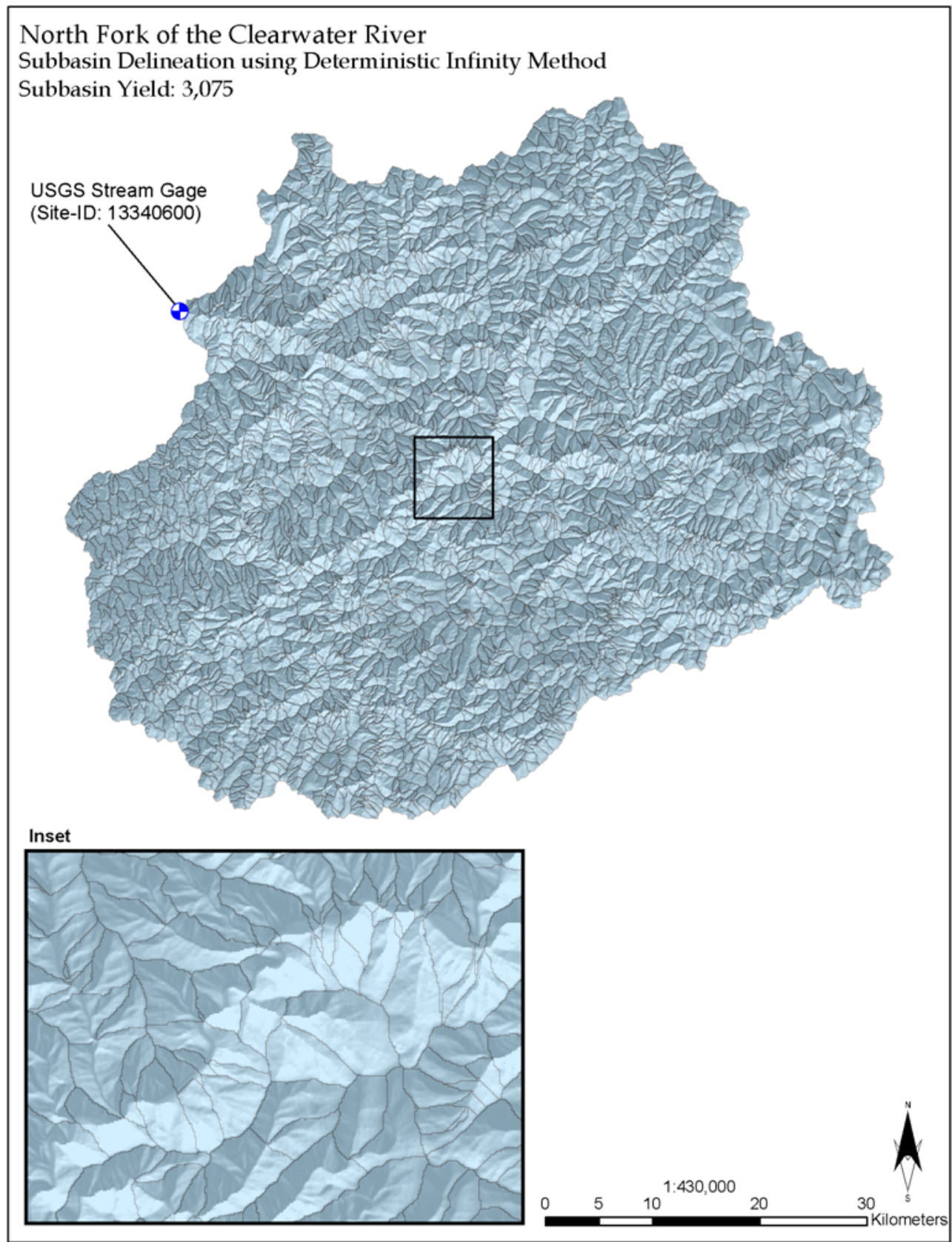

Figure 4.7. The sub-watersheds presented in this figure serve as a spatial container for data harvesting and compilation of data exhibiting multiple-scales and data types. 


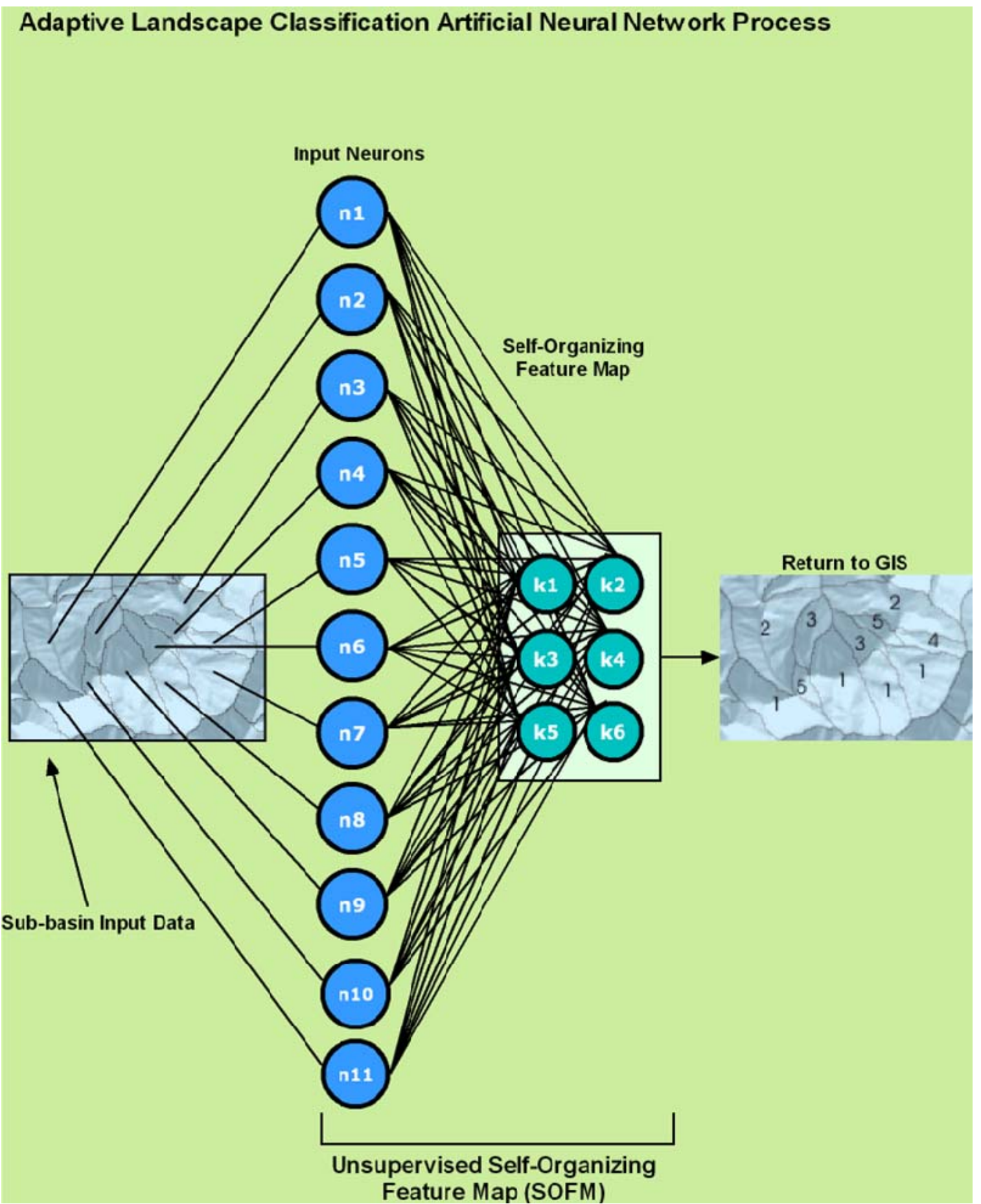

Figure 4.8. The basic process flow and function of the spatial container (as represented by subwatersheds) within the ALCP. The input neurons are representative of the vector codebook patterns containing the multiple datasets that are used in the classification. 


\subsubsection{SOM Pre-Processor}

While many versions of the SOM exist, the basic construct is the same, although the implementations may vary. For example, some models are capable of projecting data to 2-D space, while others support 2-D and 3-D topologies of varying forms (e.g., cubic, torroidal, spherical). Another difference is found in the way that data are formatted for input into the SOM model. The way Kohonen's original SOM_PAK (Kohonen et al., 1996) software reads data, differs from the SOM implementation in the ALCP, which is discussed in Section 4.5.

The SOM pre-processor functions within the GIS system, accessing the spatial database records created for each spatial container, then dumps these records into a simple ASCII table format that takes on the following tab-delimited form:

$$
\text { ID } \quad A_{1} \quad A_{2} \quad \ldots \quad A_{n} \quad \text { ATTR }
$$

where, $I D$ is a unique numeric identifier of each spatial container, $A_{n}$ are the numerically descriptive parameters of the individual datasets included for classification, and ATTR is an optional alphanumeric attribute field if any tags are needed to help identify a characteristic of the spatial container. If we were to take a look at a simple example of classifying a —cyan, magenta, yellow, and black(CMYK) color table, where four parameters represent the magnitude of each color, the SOM input file would look like this:

$\begin{array}{llllll}1 & 0 & 0 & 51 & 0 & \mathrm{~A} \\ 2 & 0 & 0 & 79 & 0 & \mathrm{~A} \\ 3 & 0 & 1 & 95 & 0 & \mathrm{~A} \\ 4 & 0 & 3 & 100 & 18 & \mathrm{~A} \\ 5 & 0 & 3 & 100 & 30 & \mathrm{~A} \\ 6 & 0 & 3 & 100 & 50 & \mathrm{~A} \\ 7 & 0 & 2 & 69 & 0 & \mathrm{~A} \\ 8 & 0 & 4 & 79 & 0 & \mathrm{~A} \\ 9 & 0 & 6 & 95 & 0 & \mathrm{~A}\end{array}$

The source CMYK table contains 1200 unique CMYK combinations that can be presented to the SOM and the expected results would contain a mapping of CMYK colors into logical color groupings, similar to what was demonstrated in Chapter 3. 


\subsection{SOM Model and Post-Processor}

In providing the core strength of the ALCP, the SOM model relies on the strengths of neurocomputing, dimensionality reduction, and competitive learning to enable pattern-matching capability to learn similarities in the data. Details of the SOM algorithm can be found in Section 3.4.2.1. After experimentation and evaluation with several SOM implementations, including SOM PAK (Kohonen et al., 1996), SOM Toolbox for MatLab (Vesanto et al., 2000), MatLab's Neural Network Toolbox (Demuth and Beale, 2007), JOONE (Marrone, 2007), Geo-SOM (Bacao et al., 2004) and NeuroSolutions (Principe and Lefebvre, 2007), an open-source software project, SOMMER, from The Molecular Design Laboratory at Johann Wolfgang GoetheUniversity in Frankfurt, Germany, was selected for its usability, functionality, visualization, and feasibility for modifying the Java source code to meet the needs for the GIS interaction (Schmuker et al., 2007). The algorithm base for SOMMER stems from the DemoGNG work of Loos and Fritzke (1998). The Databionic ESOM (Emergent SOM) software, from the University of Marburg, Germany, was not evaluated because it was discovered after the development of a working solution with SOMMER; however the software appears to have the required capabilities, and could offer potential for future research as there are extended and unique capabilities offered.

SOMMER is an application written in the Java programming language, so it has the capability to run on multiple platforms, including Windows, Linux, UNIX, and Mac OS $X$. Four topologies are available for SOM training, each with it own adjustable parameters:

- rectangular (2-D)/XY grid

- $\quad$ cubic (3-D)/XYZ grid

- toroidal (2-D)/rectangular topology with wrapped edges

- spherical (3-D)/tessellated sphere.

In addition, other SOM training parameters such as Euclidean/Manhattan distance function, initial/final neighborhood size, initial/final learning rate, epochs, and training method are specified. While SOMMER is most typically run in a graphical mode, personal communications with the author of SOMMER uncovered an undocumented feature for specifying command-line execution of SOMMER on a previously trained 
SOM model; however, there was no capability to train a SOM by command-line execution, which would be required for seamless interaction with the GIS system. Because SOMMER is open-source software and discussions with the author of SOMMER supported the idea of SOM training by command-line, or batch mode, modifications were made to the original Java source code and to develop the concept for a parameter file specification to be passed to SOMMER for training. The updated code has been checked in to the Concurrent Versions System (CVS) software versioning system on the project home page (http://sourceforge.net/projects/sommer/) and is available for use by others.

The following specifies the updated command-line execution of SOMMER for SOM training:

java -jar sommer.jar--training-param-file input.dat

where--training-param-file is a file with the following specifications:

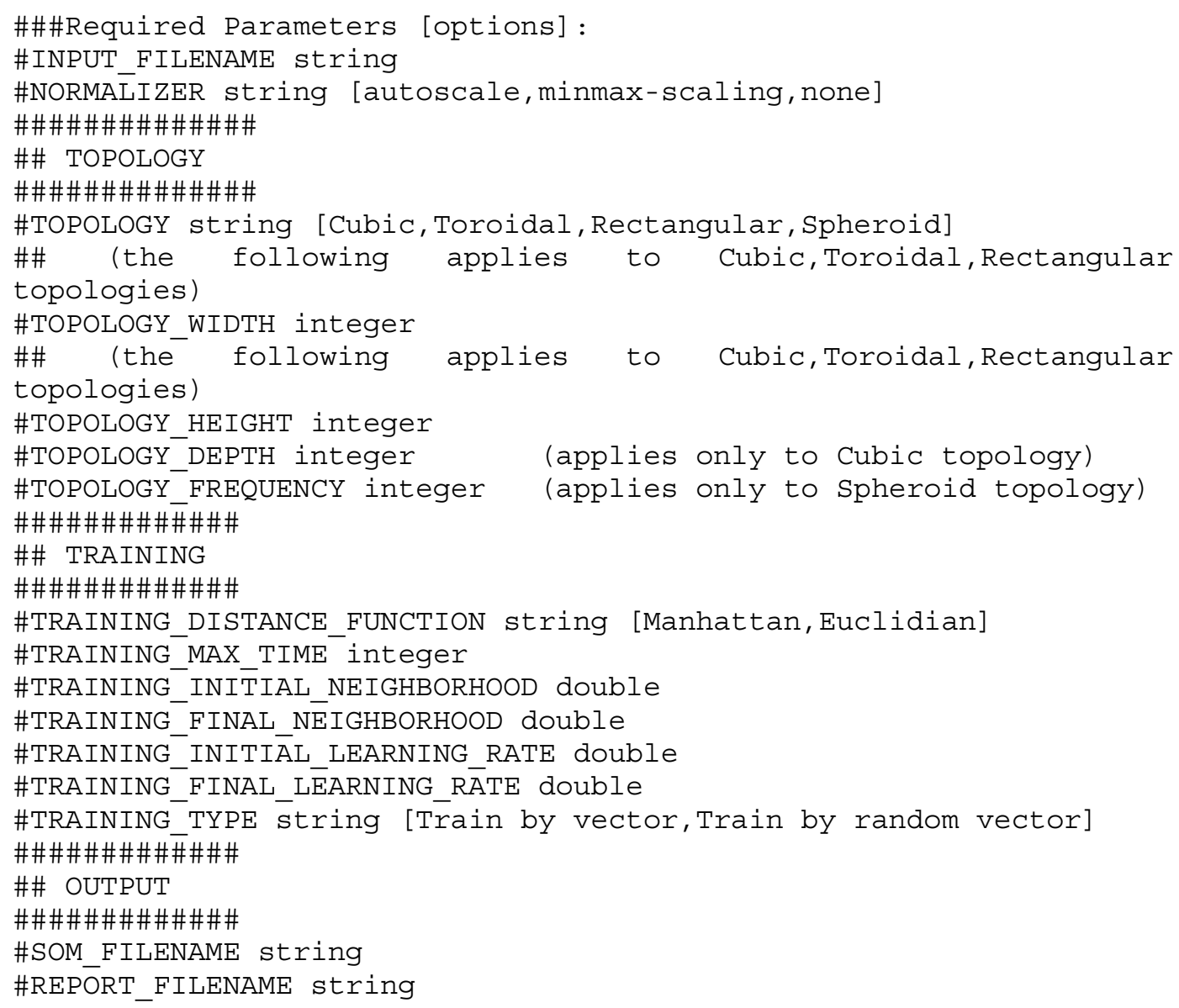


Normalization of the input data structure is a procedure that is recommended to increase the accuracy of the resulting clustering (Kohonen, 2001). If there are large values in the input data and the data aren't normalized, the clustering will tend to be biased toward these large values and won't reflect the true relationships in the data. The normalization process is a preliminary step before the SOM training convenes. There are two methods within SOMMER for normalizing data. The first method is a simple Minimum / Maximum scaling, and the second is "Autoscaling," which scales the input data to unit variance then computes a Principal Component Analysis (PCA) on the first three principal components. The final result is a normalized dataset written to a new file.

Once the data normalization is completed, the SOM training begins. The parameters used in the SOM will likely require some experimentation to understand how the organized data are represented in the final output. SOMMER provides a final report, in ASCII format, detailing the neuron/input vector memberships as well as a solution performance measure, indicated by the quantization error. This measure of error is a standard metric in SOM and fundamentally presents the mean distance between the input vector data and the neuron placement. The lower the quantization error value, the stronger the SOM solution is. An example of the SOMMER output report is provided below.

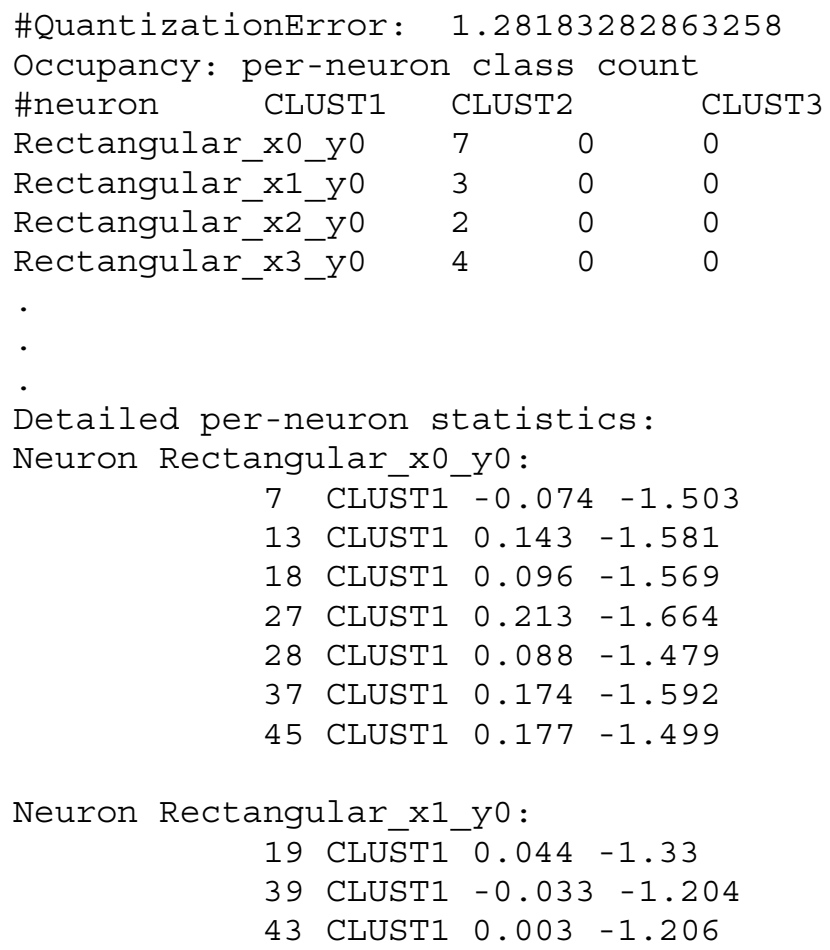


The output report is broken into two sections; the first lists a frequency count for each neuron and class (if defined), and the second section gives detailed information about the data vector membership to each neuron. This membership is related by the neuron ID, $x 0 \_y 0, x 1 \_y 0$, etc., and the vectors are referenced by the unique identification numbers (IDs) assigned to the spatial containers. Using the sample output provided above, relating spatial container IDs $7,13,18,27,28,37$, and 45 to neuron $x 0 \_y 0$ provides the GIS the information required to group these particular spatial containers together as one class. To clarify this point, every neuron represents a data cluster, with the exception of those neurons that were not used in the process. A Python script, executed at the closing of the SOM training process, parses and formats the output SOM file into a simple ASCII table, which contains the spatial container ID and the neuron assignment. The resulting GIS-ready table is joined back into the spatial database, allowing for visualization and analysis of the resulting process.

\subsection{Visualization and Analysis}

The final step in the ALCP is to evaluate the SOM classified data in the GIS environment where numerous tools are available for visualizing and analyzing the data. The GIS environment provides an enhanced visualization capability where the SOM classified data can be viewed in a spatial environment along with other relevant datasets to help interpret and understand the results. Additionally, statistical analysis and other numerous geoprocessing tasks can be applied to the SOM data. It is important to keep in mind that the SOM will define boundaries within the data presented to it; however, it will not assign a classification label to each of these groupings. This is, in effect, an unsupervised classification, so the interpretation of the different classes is left to the analyst working with the data, and class definitions will vary depending on the intent of the classification and the data used to conduct the classification. For some applications, simply knowing where the changes in the landscape occur may be enough. For example, this process can be used as a computational efficiency tool in the case of developing calibration parameters for a model that uses spatial information. In this case, model calibration parameters can be estimated for each SOM-determined class, rather than for each spatial container. Another example may be to use the SOM class boundaries to implement groundlevel instrumentation or monitoring such that the changes in the landscape, and thus the underlying process, are being captured in an efficient and effective manner. 
It may be advantageous to run the SOM portion of the ALCP multiple times to ensure that the data classes are produced repeatedly. The fact that SOMs start their initialization process with a set of randomly generated weights (see Figure 3.8) may cause some slight variation in the class boundaries, and it is certainly worth evaluating any boundary changes within each SOM run. The use of different variables to be included in the SOM processing also will help to gain a perspective of what data types have effects on the classification process; this process is also known as "data sensitivity analysis." For example, if the removal of a data variable from the SOM processing does not change the overall result, with additional validation, one may be able to suggest that this variable is not an important factor in the problem set being evaluated. Chapter 5.0 will demonstrate the application of the ALCP with different datasets and different scenarios enabling the reader to gain an understanding of possibilities that exist within this process. 


\subsection{Application of the ALCP}

This chapter represents a significant effort to test, exercise, and demonstrate the concepts and theories of the ALCP as discussed in Chapter 4.0. Its objective is to exhibit the adaptability of the ALCP for addressing and handling various types of data and problem sets. Through the example problems presented, an effort is made to validate the results of the ALCP by comparing outcomes to other data or by using other forms of analysis. This chapter describes three overall problem sets: 1) multispectral classification, 2) 30-year annual mean climatology, and 3) hydrologic properties and landscape characteristics, which are further broken into flow exceedence, flood frequency, and landscape analysis for predicting hydrologic properties. Each problem set includes its own discussion concerning source data, data parameters, methodology, results, and analysis of the results.

\subsection{Multi-Spectral Classification}

An application test was conducted using data from the Landsat Multi-Spectral Scanner (MSS) satellite sensor operated by the United States National Aeronautics and Space Administration (NASA). In this initial case, the full procedure of the ALCP was not used, but is included here to demonstrate the core SOM capability of the ALCP in a spatial context. The data were acquired from the University of California Irvine Machine Learning Repository ( $\mathrm{UCl}, 2007)$ and the purpose of this dataset is to use the four MSS spectral bands in a $3 \times 3$ kernel neighborhood to predict the source or kernel pixel, $X$ (see Figure 5.1). Each 80-m pixel in the dataset was determined by field observation and classified into one of seven categories (see Table 5.1). A total of 6435 individual pixels was used in the analysis and input data were structured such that each input codebook vector contained 36 values (4 spectral bands $\times 9$ neighborhood pixels). It should be noted that the field-determined classification value for each pixel was withheld from the input data, thus no a priori knowledge is included in the source data. The $36 \times 6435$ data matrix presented to the SOM is structured as follows, where PixelID is an arbitrary index number, $P 1 \ldots P 9$ represent each of the nine neighborhood pixels, and the subscript numbers ${ }_{1-4}$ represent the four spectral-bands for each of the nine pixels. 
PixelID, $P 1_{1}, P 1_{2}, P 1_{3}, P 1_{4}, \ldots P 9_{1} P 9_{2}, P 9_{3}, P 9_{4}$

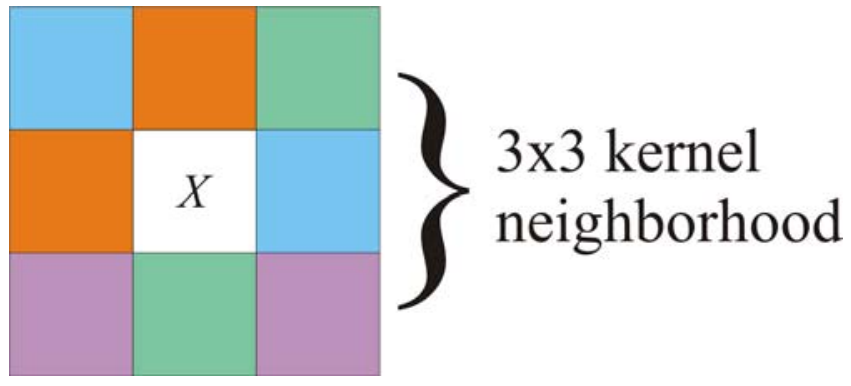

Figure 5.1. Raster-based $3 \times 3$ kernel neighborhood, where $X$ represents the kernel pixel.

Table 5.1. Classification number and descriptions of the Landsat MSS dataset.

\begin{tabular}{|l|l|}
\hline Class Number & \multicolumn{1}{|c|}{ Class Description } \\
\hline 1 & Red soil \\
\hline 2 & Cotton crop \\
\hline 3 & Gray soil \\
\hline 4 & Damp gray soil \\
\hline 5 & Soil with vegetation stubble \\
\hline 6 & Mixture class (assorted vegetation) \\
\hline 7 & Very damp gray soil \\
\hline
\end{tabular}

Twenty different SOM batch files, each containing different parameter sets, were produced and run. The intention was to experiment with varying SOM parameters (i.e., topologies, topology dimensions, distance functions, etc.) and use the results with the lowest quantization error. Views of the final projected SOM with class-coded winning neurons, in both its natural projection and structured representation, can be viewed in Figure 5.2. The data within the resulting SOM clusters used the individual PixelID numbers to map values back to the field-determined class number. In an ideal case, each SOM cluster would only contain pixel values from a single class number; however noise and natural variation in the data tend to preclude this, especially in more complex datasets. To understand the quality of the process and ability for the SOM to cluster groups of data into logical classes, class data for each neuron were constructed into a table, where the dominant number of class instances for a given neuron was declared the "winning class," such as is presented in Table 5.2. From this table, it is clear there are pixel values being misclassified; however, in 
general these cases seem to be low in number. To determine the total fitness of the classification process, the count of the winning class neurons was compared to the total number of pixels in the analysis, yielding $87.3 \%$ of the pixels that were properly classified. To gain further understanding of the misclassified data, primarily to evaluate possible class overlaps, classes that exhibit some similar characteristics, or resulting in data noise, a confusion matrix (Provost and Kohavi, 1998) was built (see Table 5.3).

Table 5.2. Class-assigned pixel counts for each SOM neuron are presented. The dominant count value, marked in bold-italic typeface, is declared the "class winner" for the neuron.

\begin{tabular}{|l|r|r|r|r|r|r|}
\cline { 2 - 7 } \multicolumn{1}{c|}{} & \multicolumn{7}{c|}{ Class Number } \\
\hline Neuron & $\mathbf{1}$ & $\mathbf{2}$ & $\mathbf{3}$ & $\mathbf{4}$ & $\mathbf{5}$ & $\mathbf{7}$ \\
\hline x0y0z0 & $\mathbf{4 9}$ & 0 & 0 & 0 & 2 & 0 \\
\hline x1y0z0 & $\mathbf{4 5}$ & 0 & 0 & 0 & 7 & 0 \\
\hline x2y0z0 & 0 & $\mathbf{1 1}$ & 4 & 2 & 8 & 1 \\
\hline x3y0z0 & 0 & 0 & $\mathbf{2 0}$ & 3 & 0 & 2 \\
\hline x4y0z0 & 1 & 0 & $\mathbf{3 5}$ & 6 & 0 & 5 \\
\hline x0y1z0 & $\mathbf{7 9}$ & 0 & 0 & 0 & 0 & 0 \\
\hline x1y1z0 & $\mathbf{7 0}$ & 0 & 0 & 0 & 0 & 0 \\
\hline x2y1z0 & $\mathbf{1 1}$ & 0 & 0 & 1 & 1 & 0 \\
\hline x3y1z0 & 0 & 0 & 2 & 14 & 0 & $\mathbf{2 7}$ \\
\hline x4y1z0 & 0 & 0 & 10 & 12 & 0 & $\mathbf{1 5}$ \\
\hline
\end{tabular}

Table 5.3. A confusion matrix showing classified and misclassified data by class. Values indicated by bold-italic typeface indicate correctly classified values. All data are presented as percentages.

\begin{tabular}{|r|r|r|r|r|r|r|}
\hline & $\mathbf{1}$ & $\mathbf{2}$ & $\mathbf{3}$ & $\mathbf{4}$ & $\mathbf{5}$ & $\mathbf{7}$ \\
\hline $\mathbf{1}$ & $\mathbf{9 6 . 1 9}$ & 0.29 & 1.09 & 0.00 & 2.07 & 0.00 \\
\hline $\mathbf{2}$ & 0.00 & $\mathbf{9 5 . 8 5}$ & 0.00 & 0.46 & 2.81 & 0.39 \\
\hline $\mathbf{3}$ & 1.42 & 0.57 & $\mathbf{8 6 . 6 8}$ & 6.02 & 0.15 & 1.95 \\
\hline $\mathbf{4}$ & 0.06 & 1.00 & 9.11 & $\mathbf{6 9 . 2 1}$ & 2.22 & 10.29 \\
\hline $\mathbf{5}$ & 2.33 & 2.15 & 0.27 & 0.46 & $\mathbf{8 3 . 7 0}$ & 3.58 \\
\hline $\mathbf{7}$ & 0.00 & 0.14 & 2.86 & 23.84 & 9.04 & $\mathbf{8 3 . 7 8}$ \\
\hline
\end{tabular}

The confusion matrix reveals very good classification results for classes 1 (red soil) and 2 (cotton crop), both yielding a 96\% correct classification. Classes 3 (gray soil), 5 (soil with vegetable stubble), and 7 (very damp gray soil), were also well grouped, ranging from $83-86 \%$ correct results. Class 4 (damp gray soil) yielded a $69 \%$ correct classification and $24 \%$ of the pixel data lumped under Class 4 actually belonged to Class 7 (very damp gray soil). If we evaluate the results from Class 7 (very damp gray soil), it is evident that $10 \%$ of the Class 4 (damp gray soil) pixels were incorrectly classified. It is clear that some cases of uncertainty exist between Class 4 (damp gray soil) and Class 7 (very damp gray soil), either in the source data or perhaps in the interpretation of the field data. It is also possible that this is a case of class overlap, where within the $3 \times 3$ kernel neighborhood both "damp" and "very 
damp" conditions in the gray soils were present. Also notable is the fact that $9 \%$ of Class 4 (damp gray soils) values were misclassified under Class 3 (gray soils), $6 \%$ of Class 3 (gray soil) values were misclassified under Class 4 (damp gray soils), and $9 \%$ of Class 7 (very damp gray soil) values were misclassified under Class 5 (soil with vegetation stubble).
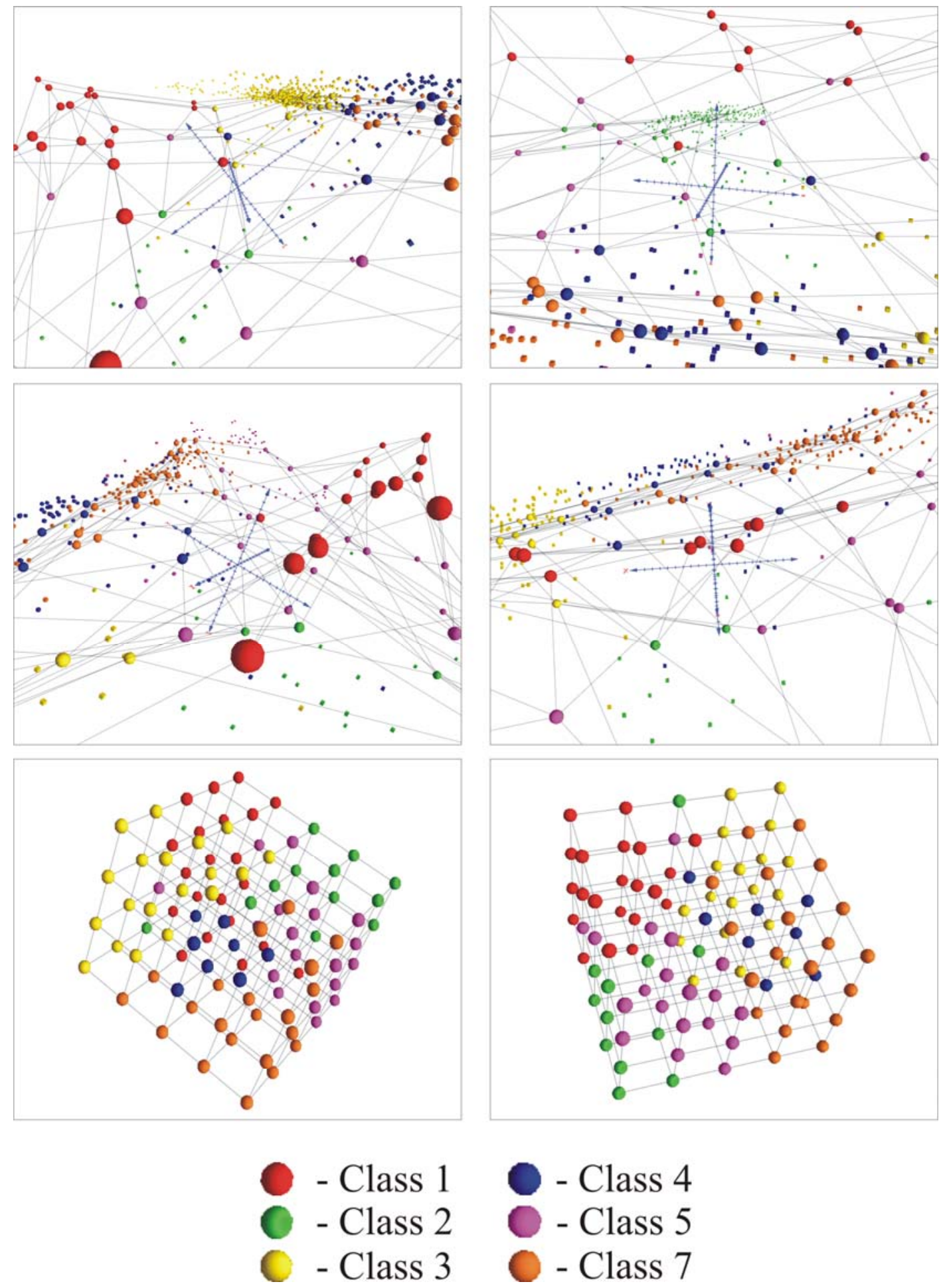

Figure 5.2. The upper four figures display the Landsat MSS input data space (represented by classcolored cubes) and the final projected SOM neurons (represented by class-colored spheres). The bottom two figures display the final projected SOM in structured space. 


\subsection{0-Year Annual Mean Climatology}

To test the ALCP in its full procedural capacity, a reasonably simple dataset with limited parameters of 30-year annual mean maximum temperature, minimum temperature, and precipitation was tested. The test watershed for this exercise was the North Fork of the Clearwater River. This watershed is a $\sim 3,300-\mathrm{km}^{2}$ area located within the greater Snake and Columbia River watersheds and is characterized by mountainous and rugged terrain with elevations ranging from $500-2400-\mathrm{m}$. This area exhibits a dominant land cover of coniferous forest and the hydrography is generally an east-to-west flowing system with high-gradient streams. The watershed is influenced by orographic precipitation events sourced from Pacific Ocean based moisture where the long-term mean annual precipitation ranges from $700-\mathrm{mm}$ in the lower elevations to 1,650-mm in the upper reaches of the basin (NRCS, 2007; WRCC, 2007). The spatial containers for this exercise are terrain-modeled subbasins that were extracted using a $10-\mathrm{m}$ resolution DEM and the $\mathrm{D}_{\infty}$ method (Tarboton, 1997) to extract the sub-basin watershed boundaries (see Figure 5.3).

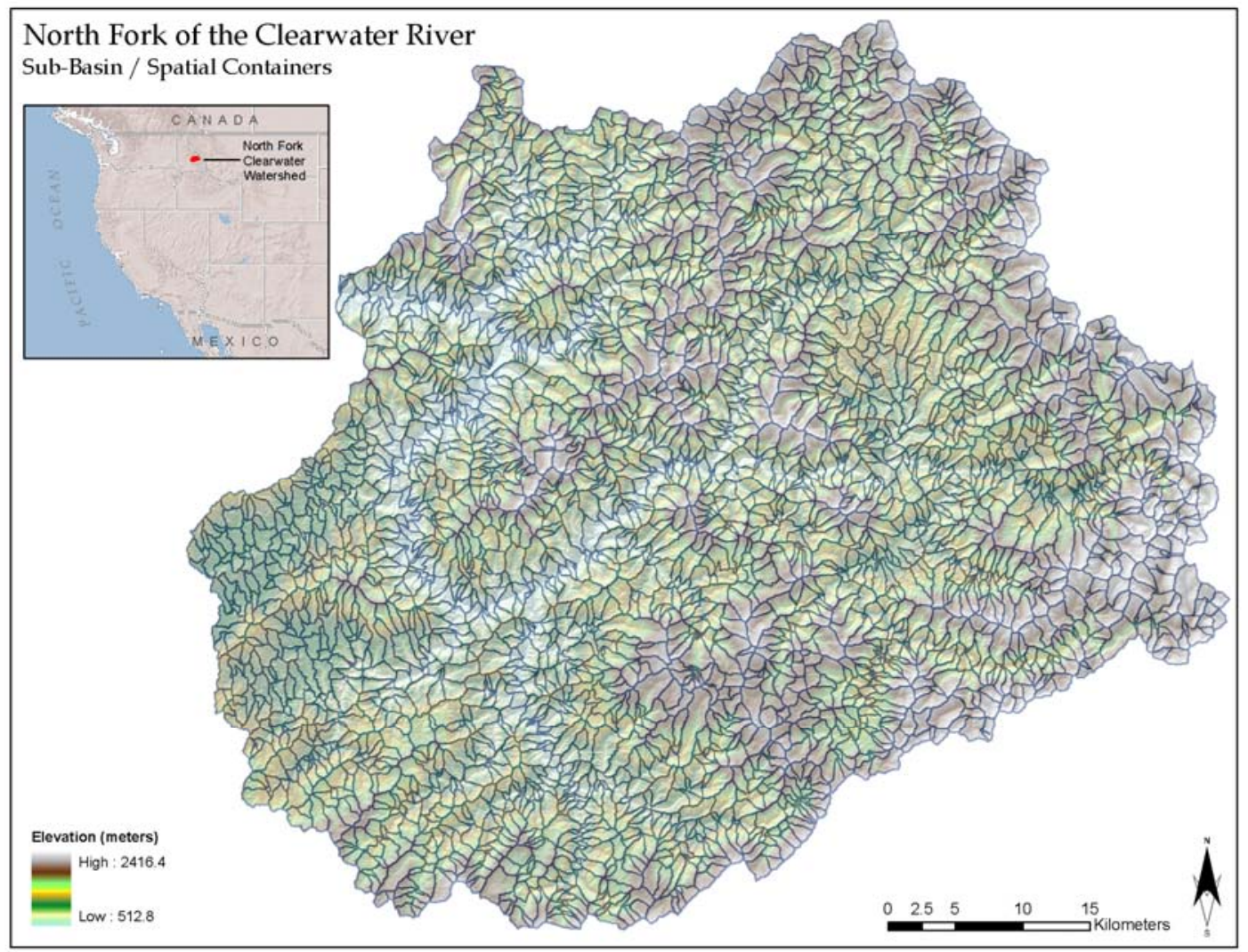

Figure 5.3. An overview of the 3075 ALCP spatial containers used for analysis on the North Fork of the Clearwater River watershed. 
The 30-year annual climate data were derived from the Parameter-elevation Regressions on Independent Slopes Model (PRISM) dataset available from Oregon State University (Daly et al., 1994). The PRISM dataset provides a distributed grid of data at a resolution of 800-m and covers an analysis period from 1971-2000. PRISM data are sourced from in situ weather station data and several meteorological influencing phenomena such as off-shore oceanic conditions and topography. The ALCP process sampled each source dataset (i.e., minimum/maximum temperature, precipitation) for each of the 3075 spatial containers present in the test dataset. A SOM input file was then built with the following structure resulting in an input data matrix of $4 \times 3075$ :

$$
I D, T_{\min }, T_{\max }, P_{\text {mean }}
$$

A 3-D cubic topology $(2 \times 2 \times 2)$ neuron structure, which clusters the data into one of a possible eight categories, was chosen for this SOM analysis. The low neuron count (i.e., clusters) was chosen for two reasons. First, a smaller level of classification would allow an easier understanding of how the ALCP would spatially distribute the classified climate data, thereby deriving the simple benefit of spatially evaluating whether or not the process appears to make sense. The second reason is to answer a question concerning the best placement of new meteorology collection stations to supplement three existing stations. The United States Army Corps of Engineers, operator of a hydroelectric dam below the analysis watershed, recently posed a question about where to best locate new stations to gain a better representation of the meteorology, which allows for better characterization and planning of water supply, water quality issues, flood events, environmental constraints, and hydropower operations.

The ALCP process results can be found in Figure 5.4 and Figure 5.5, which show the projected SOM and the input data space, and the resulting data clusters mapped back to the GIS environment, respectively. Broadly evaluating the spatial results, it is apparent that the ALCP process determined patterns in the meteorology that subsequently reveal patterns in the underlying elevation in the watershed (see the underlying topography in Figure 5.5 for comparison). Keep in mind, the ALCP had no knowledge of the topographic structure or elevation in the watershed, only three elements of meteorological data. It is possible that the climate/elevation relationship is revealing one the major parameters used in the PRISM model, which is the source 
of the distributed climate data used in this analysis. Consider as well, elevation is a major driver in weather events, leading to orographic precipitation effects and typically a decrease in temperature with an increase in elevation.

(a)

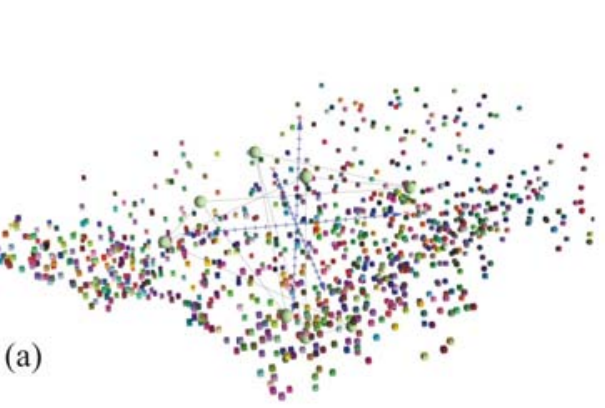

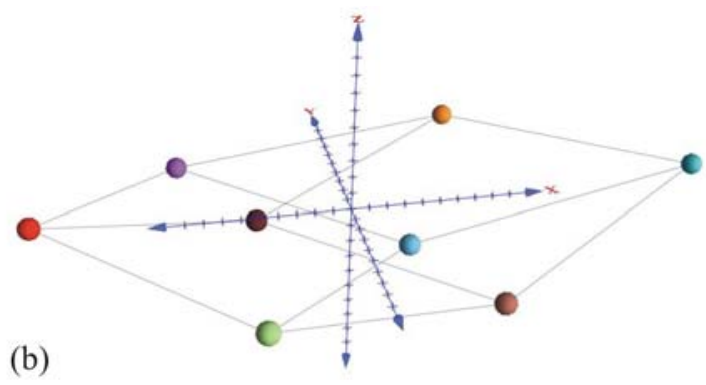

Figure 5.4. The input data space (colored cubes) and random-weighted neurons (spheres) for the North Fork Clearwater three-parameter climate data are represented in (a). The final projected SOM, a three-dimensional 2x2x2 cubic topology network, is presented in (b).

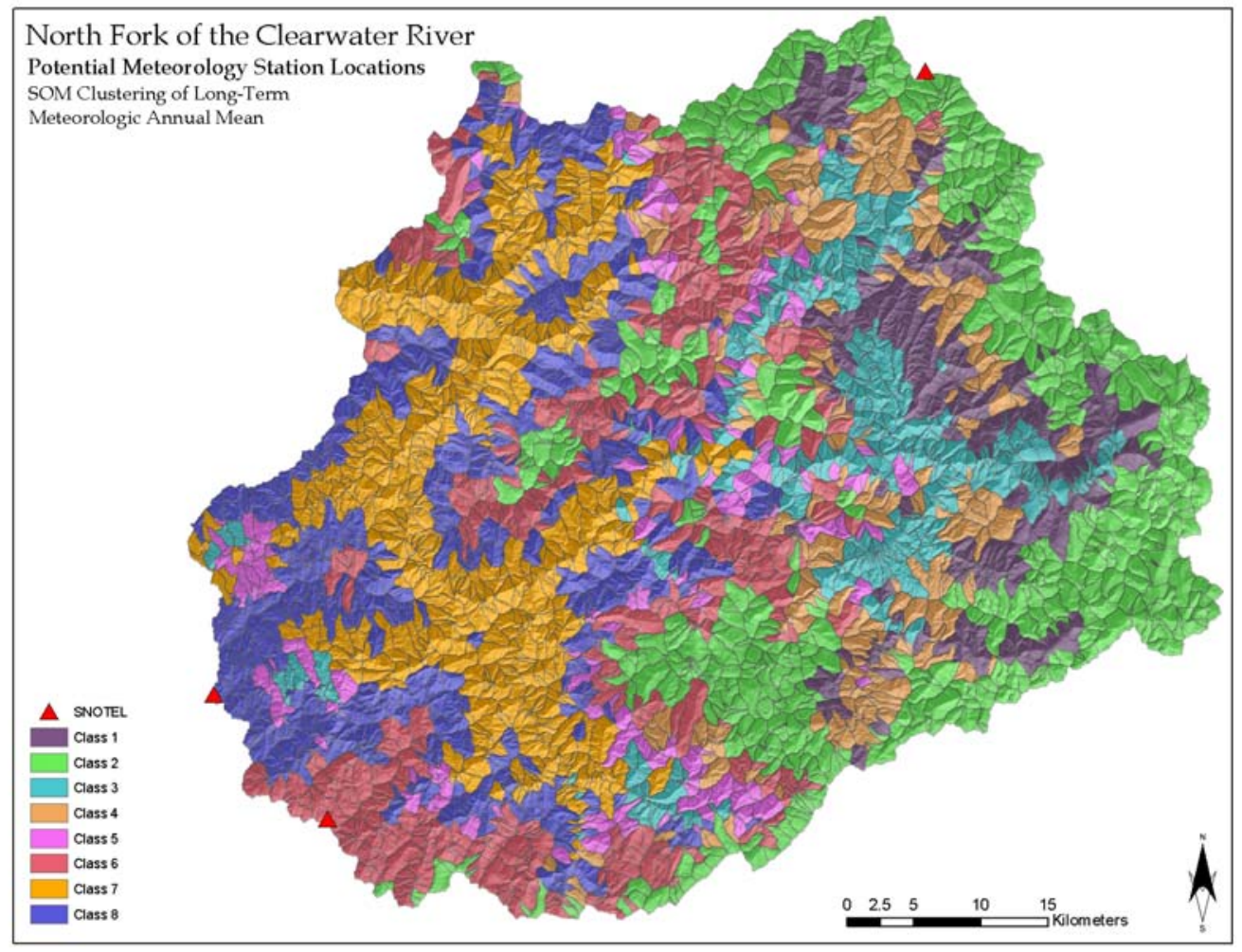

Figure 5.5. The ALCP analysis/spatial classification of 30-year annual mean climate data in the North Fork of the Clearwater watershed. Existing meteorology stations are noted with the red triangles.

A regression analysis (see Figure 5.6) was completed to help understand the relationship of elevation to each of the three climate elements presented to the ALCP, and thus attempt to explain the elevation-related patterns visible in Figure 5.5. Strong correlation coefficients were found for precipitation and elevation $\left(R^{2}=0.76\right)$, and for maximum temperature and elevation $\left(R^{2}=0.96\right)$. The correlation coefficient 
for minimum temperature and elevation resulted in a lower relationship value at $\mathrm{R}^{2}=0.36$. Thus, two of the three elements used for this application have strong ties to elevation, which appears to influence the SOM cluster process; however, the weaker relationship with elevation and minimum temperature reveals that other factors come into play and further study of the PRISM model would likely help to understand them.

Statistical analysis was conducted on the individual data elements by the eight class divisions determined by the ALCP process. Basic data properties such as minimum value, $25^{\text {th }}$ percentile, median value, $75^{\text {th }}$ percentile, maximum value, standard deviation, and mean value were derived for three elements for each of the eight classes. The resulting data were plotted into three box-and-whisker plots presented in Figure 5.7. Upon examination of the box-and-whisker plots, on an individual element basis, there are cases where it appears some classes could be merged because of a similarity between data values, or in some cases, values in a certain class could easily fit within the bounds of another class. However, further examination of the other elements reveals differences in these same classes, thus the overall class separation becomes apparent and justified.

Finally, to address the question posed concerning the placement of new meteorology collection stations, using the assumption that a larger representative land area would better characterize conditions in the watershed, class boundaries were plotted based on area and are presented in Figure 5.8. The four dominant class zones are represented by Classes $2,6,7$, and 8 , making up approximately $70 \%$ of the total watershed area. Within three of these dominant classes (Classes 2, 6, and 8), which collectively represent $53 \%$ of the total watershed area, meteorology stations already exist. Adding an additional station within the boundaries of Class 7 can increase the representative area by $17 \%$ and has an added benefit of representing a lower elevation zone (the existing stations are positioned along the watershed boundary at the highest elevation zones). While this analysis is presented to apply and demonstrate the ALCP, a more detailed analysis of this same process was completed (Coleman and Vail, 2007) at monthly mean time scale to refine station placement, considering monthly meteorological variations and identifying and isolating those periods of the year where extreme meteorological events occur. 

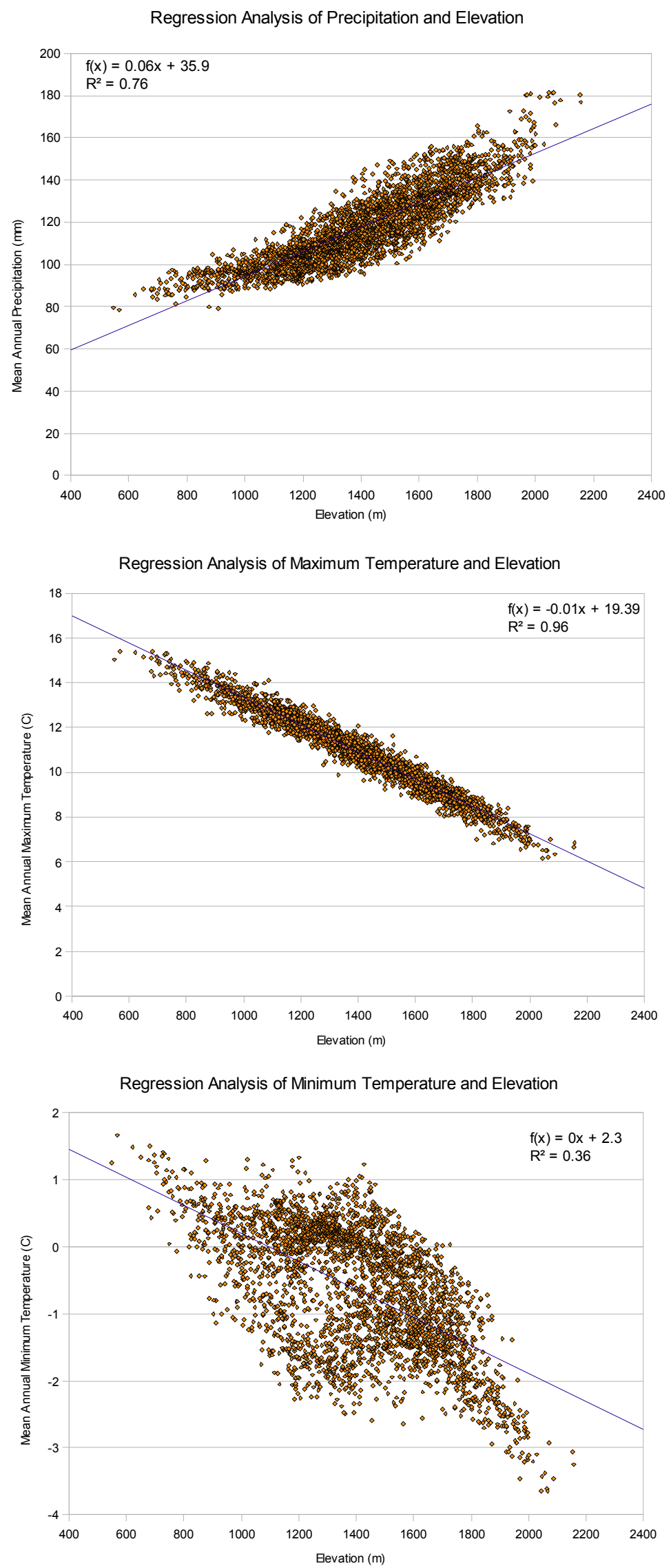

Figure 5.6. Linear regression analysis evaluating the relationship of elevation to precipitation, maximum temperature, and minimum temperature. 

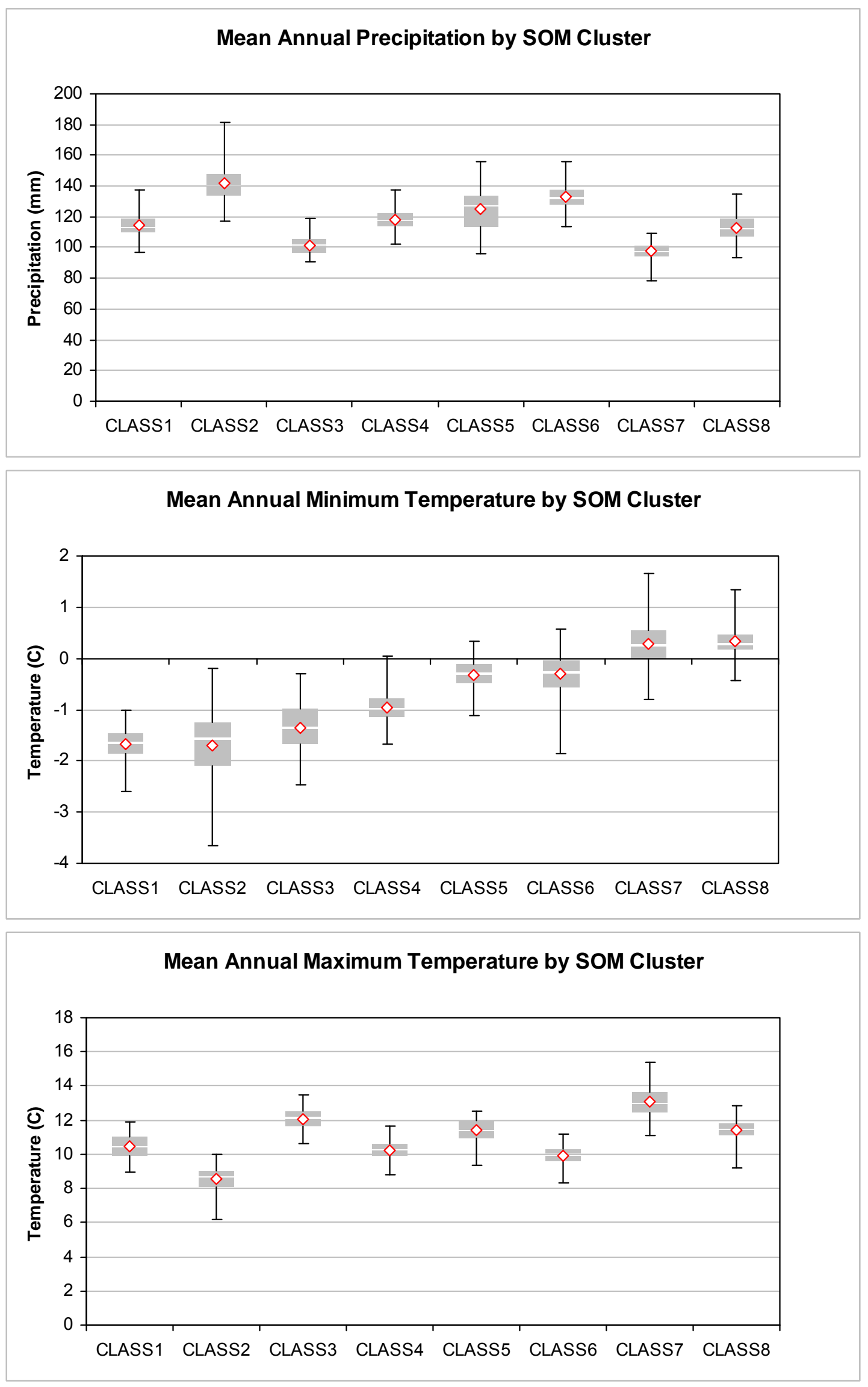

Figure 5.7. Box-and-whisker plots for precipitation minimum temperature and maximum temperature for each SOM-determined cluster. 


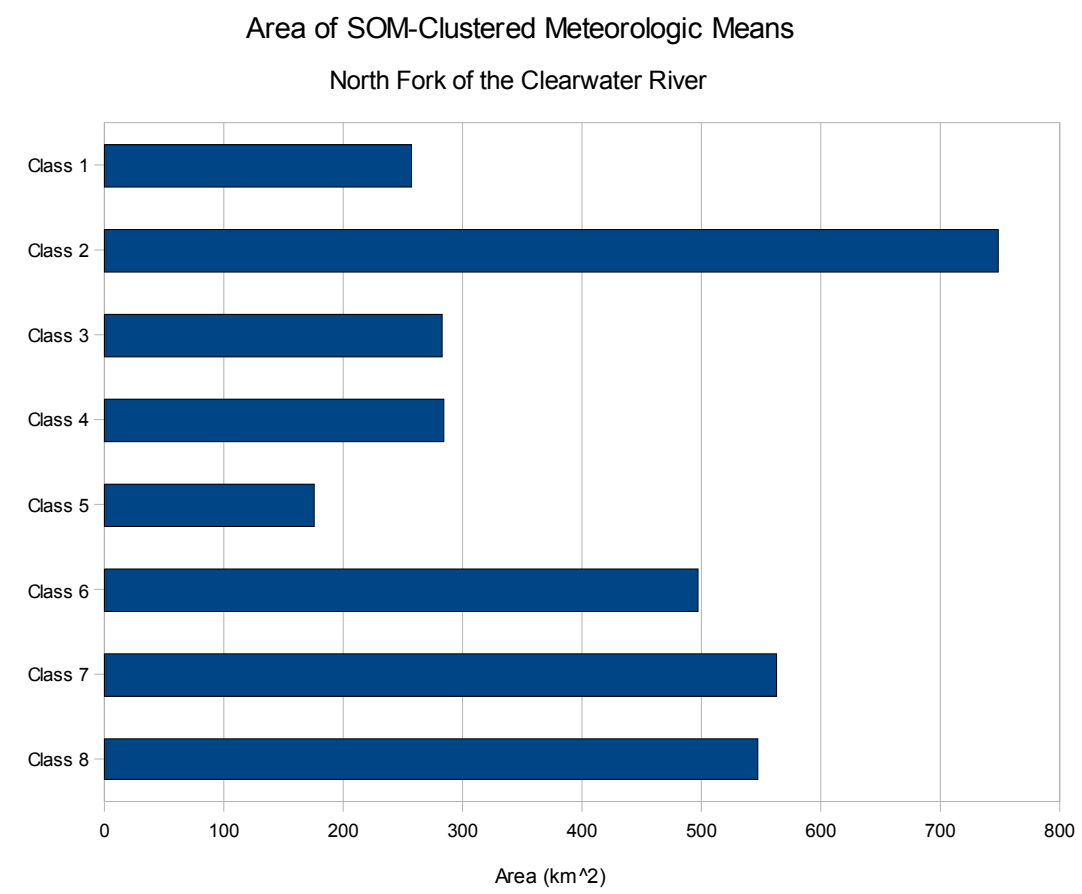

Figure 5.8. Bar graph representing the total area occupied by each SOM-clustered class as represented in Figure 5.5. Note that existing meteorology collection stations exist in Class 2, Class 6, and Class 8.

\subsection{Hydrologic Properties and Landscape Characteristics}

The hydrologic properties of a watershed are good indicators of the intrinsic landscape characteristics. For instance, watersheds that have high peak flows may be an indicator of a watershed with steep terrain, low vegetation canopy, and less permeable soils such as clay-loams. In this demonstration of the ALCP, established multivariate regression equations were used to calculate peak flood return periods, also known as flood frequencies, and flow exceedence for ungaged basins. The results of the hydrologic analysis are presented, then clustered and mapped using the ALCP. Finally, in an attempt to strictly use spatially derived landscape data, the ALCP is used to estimate its fitness for estimating hydrologic properties. This analysis not only exercises the use of non-traditional spatially derived data, but also exhibits the flexibility of the ALCP to process data sources from diverse scales and varying spatial container sizes.

A total of 160 sub-basins, covering approximately $63 \%$ of the total area in the North Fork of the Clearwater watershed, were interactively selected and derived for 
analysis (see Figure 5.9). The selected basins have an area range from $4-32 \mathrm{~km}^{2}$ suggesting the possibility for a somewhat diverse range in hydrologic properties. As recommended by the USGS, publisher of the streamflow regression equations used in this analysis (Hortness and Berenbrock, 2001), the effective minimum basin area to use in this analysis is $4 \mathrm{~km}^{2}$. The distribution of basin areas can be viewed in Figure 5.10, where the median basin area is $11 \mathrm{~km}^{2}$. It should be noted that all basins used in this analysis are headwater catchments, meaning no other channels flow into these basins; rather, they initiate stream channels and provide flow to downstream catchments.

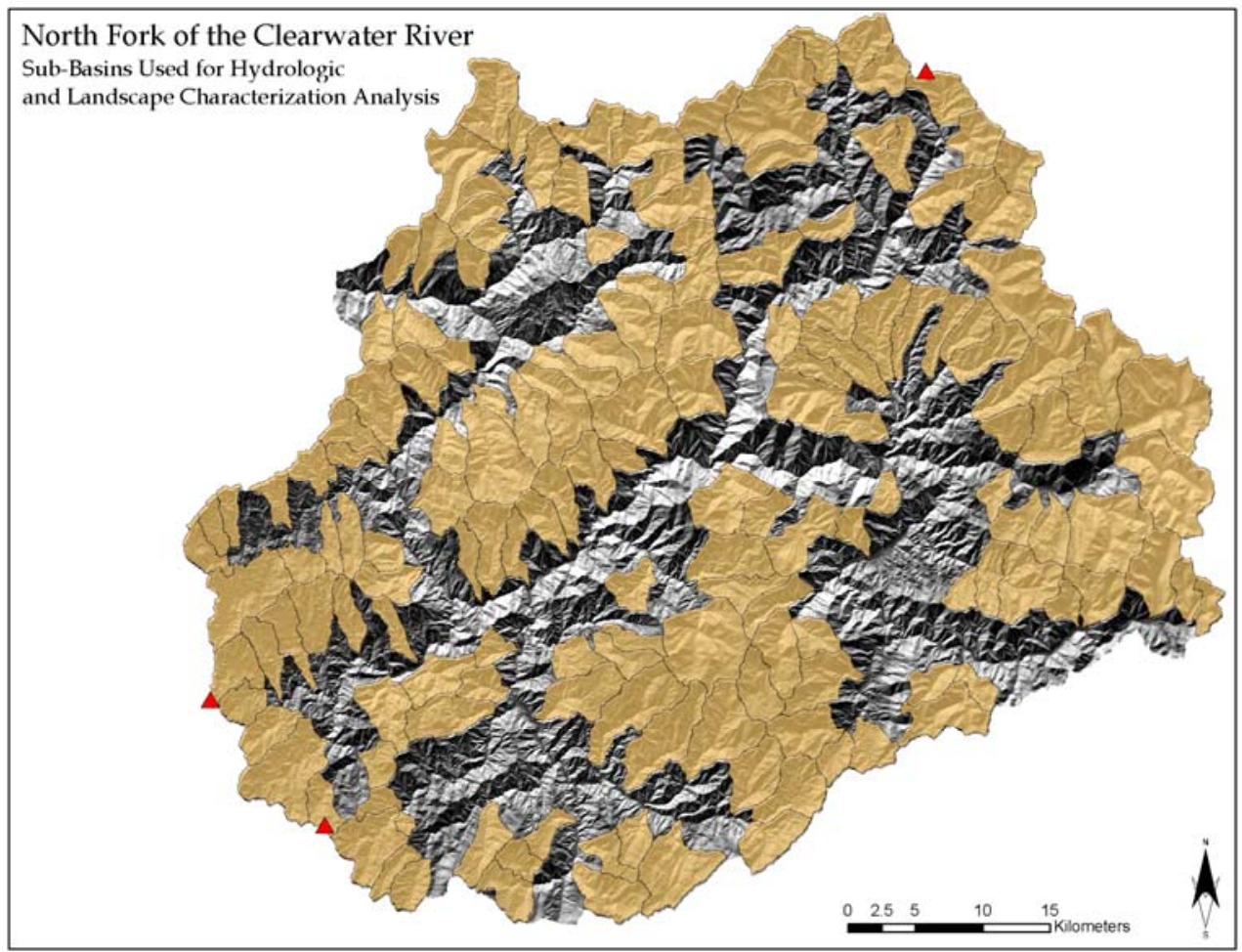

Figure 5.9. A total of 160 headwater catchments were derived for hydrologic and landscape analysis. The selected basins represent approximately $63 \%$ of the total watershed area. 


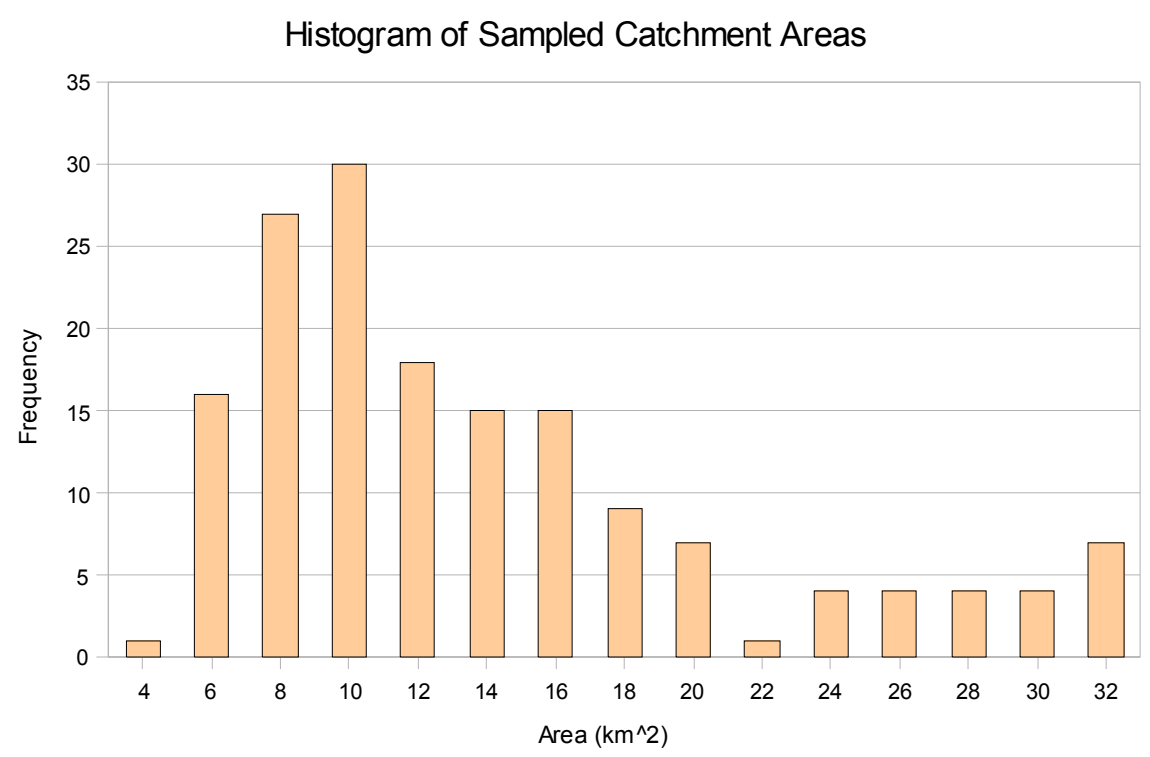

Figure 5.10. Area distribution of the 160 sub-basins sampled for analysis.

\subsubsection{Flow Exceedence Analysis}

The work of Hortness and Berenbrock (2001) aims to determine streamflow characteristics for watersheds without flow recording instrumentation (i.e., ungaged basins). The streamflow characteristics of interest are 1) exceedence values, where daily mean stream discharge is evaluated for flows that occur $20 \%, 50 \%$, and $80 \%$ of the time (i.e., $80 \%$ exceedence values are lower flows that occur more frequently and $20 \%$ exceedence values represent high flow periods that only occur $20 \%$ of the time) and 2) mean annual discharge. Building on the work of others (Horn, 1988; Kjelstrom, 1998; Lipscomb, 1998; Quillian and Harenberg, 1982), Hortness and Brenbrock (2001) used the notion of relating data from approximately 200 stream gages with at least 10 years of records to a series of basin characteristics including drainage area $(A)$, mean basin elevation $\left(E^{*}\right)$, basin relief $(B R)$, percent of area of slopes greater than $30 \%$ ( $S 30$ ), mean annual precipitation $(P)$, percent of forested area $\left(F^{*}\right)$, mean basin slope ( $B S$ ), and mean value of the main channel slope ( $M C S$ ). Separate regression equations were developed for eight broadly defined regions throughout the state of Idaho to achieve "hydrologically homogeneous" zones. Hortness and Berenbrock (2001) state that "In general, the equations are more reliable (lower standard errors of estimate) for estimating the high streamflow statistics (20-percent exceedence) than for estimating the low streamflow statistics (80-percent exceedence) in any given month." Given this statement, the final analysis of using landscape characteristics to estimate hydrologic 
properties is conducted only for the $20 \%$ flow exceedence (see Section 5.3.3). A full list of the regression equations used for the estimation of monthly flow exceedence values can be found in Appendix B; however, as an example, the equation for $20 \%$ flow exceedence in April is provided here:

$$
Q .20=1.26 \times 10^{-6} A^{0.978} E^{*^{-0.480}} F^{*^{1.87}} P^{2.10}
$$

The required spatially derived landscape parameters for the monthly flow exceedence analysis were incorporated into the ALCP to provide a similar capability for future analysis on other watersheds. Once the required landscape characteristics for all 160 test sub-basins were determined, the published multi-regression equations were used to calculate monthly $80 \%$ (Q80) and 20\% (Q20) flow exceedence. The results of this effort are shown in Figure 5.11 and Figure 5.12. The peak runoff season is clearly visible from the months of March through July where the accumulated snowpack is released to streamflow, typically hitting its peak in late April and early May. Also notable is the observation that, while the majority of the basins seem to follow a consistent trend, and it almost appears that values are scaled to a variable such as area, there are basins here that have a dampened and delayed peak flow period, suggesting the influence of other basin characteristics. 


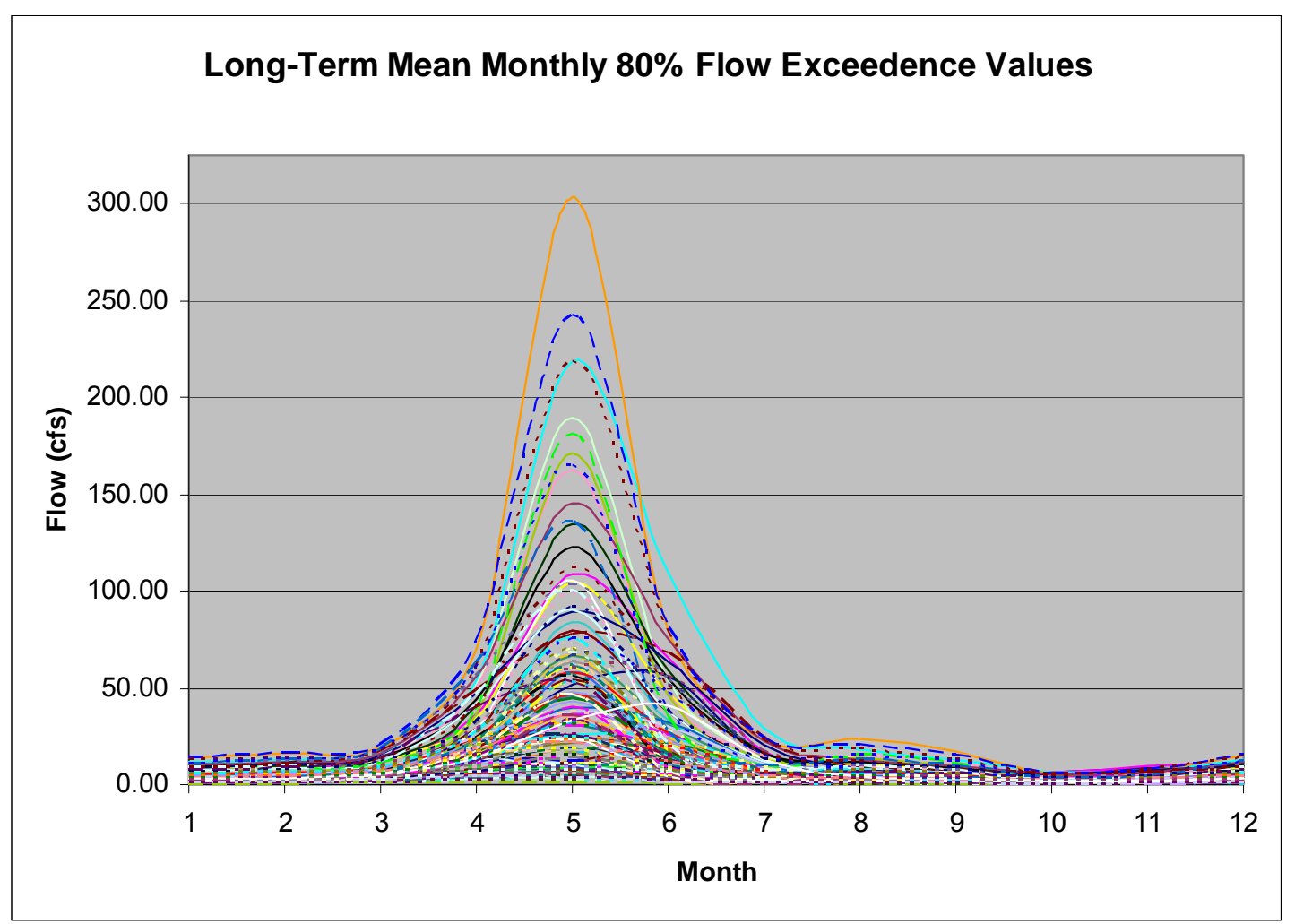

Figure 5.11. Monthly values of $80 \%$ flow exceedence for all 160 test sub-basins. Flow units are in cubic feet per second (cfs).

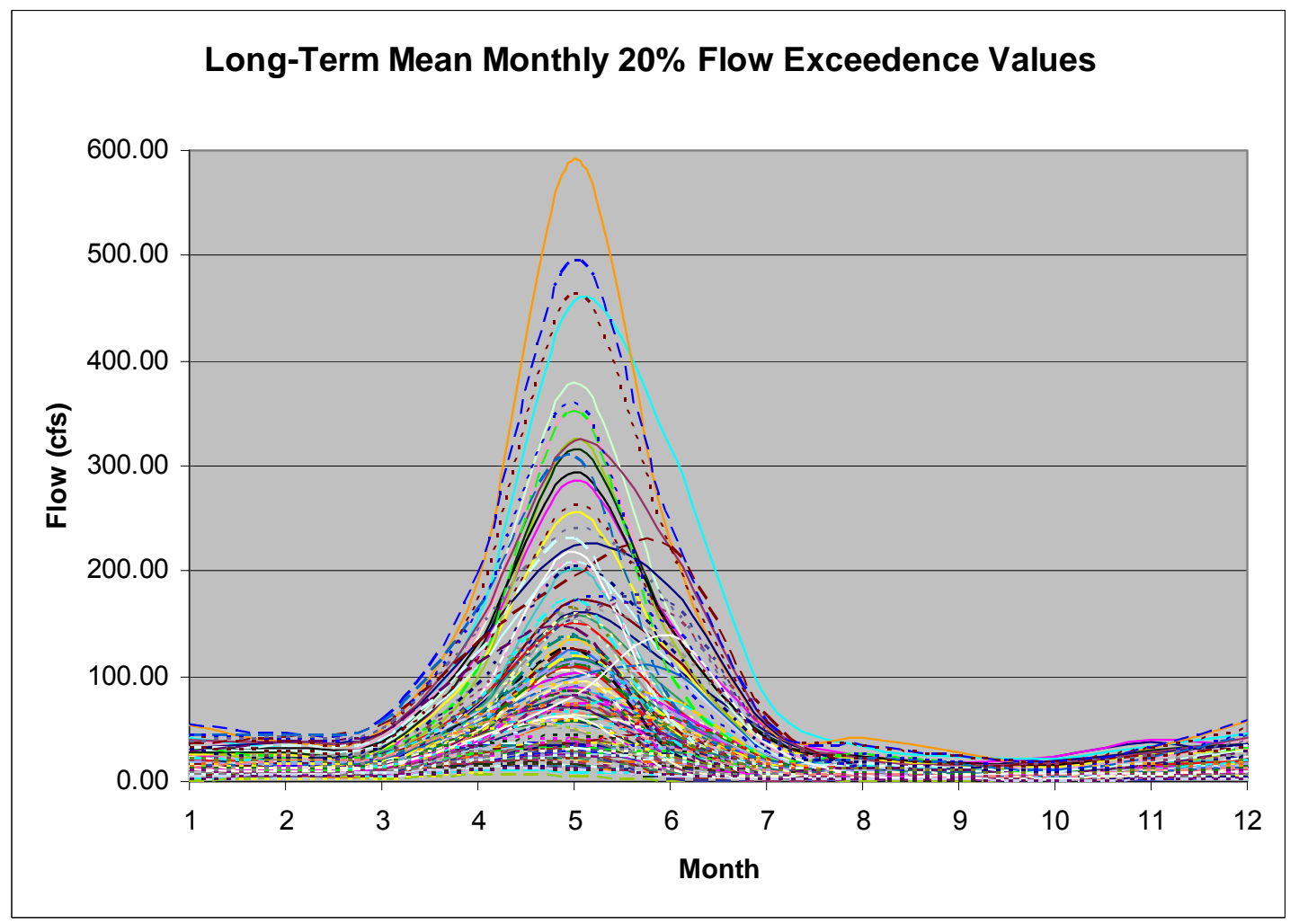

Figure 5.12. Monthly values of $20 \%$ flow exceedence for all 160 test sub-basins. Flow units are in cubic feet per second (cfs). 
The objective of this analysis is to separate and classify the different flow structures for both the Q20 and Q80 flows and map these structures back to the ALCP spatial containers. This process also demonstrates the ability of the ALCP to process and handle temporal-based signal data. A spatial similarity comparison is made between the Q20 and Q80 flows to understand what flow exceedence values were relationally similar among the test sub-basins. Because the majority of the activity in the streamflow occurs between March and July, data for this time period were isolated and used for this analysis. Two separate $6 \times 160$ matrix files were constructed (i.e., one for each flow exceedence group) taking on the following form:

$$
\begin{aligned}
& I D, Q 80_{\text {Mar }}, Q 80_{A p r}, Q 80_{\text {May }}, Q 80_{\text {Jun }}, Q 80_{\text {Jul }} \\
& I D, Q 20_{\text {Mar }}, Q 20_{A p r}, Q 20_{\text {May }}, Q 20_{\text {Jun }}, Q 20_{\text {Jul }}
\end{aligned}
$$

A 3-D cubic topology SOM with dimensions $2 \times 2 \times 2$ was used to generate eight class boundaries. The final projected SOM boundaries and input data are presented in Figure 5.13.

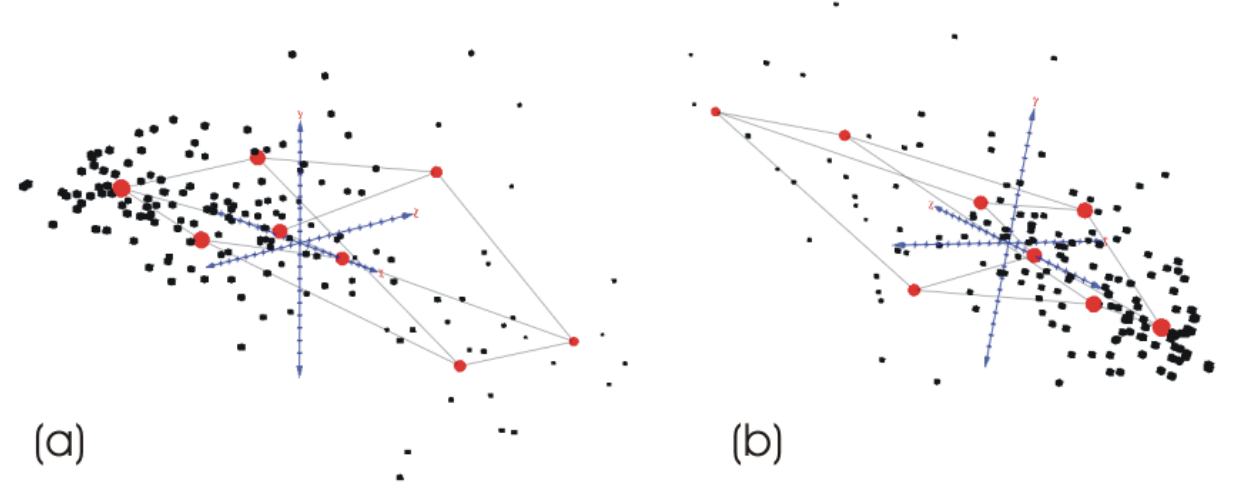

Figure 5.13. Final SOM projection for (a) Q80 and (b) Q20.

To gain a sense of the ability of the SOM process to separate the Q80 and Q20 flow structures for the peak runoff season, mean cluster values for each month from each SOM-determined class were plotted then color-coded according to overall flow values; these results are presented in Figure 5.14 From these plots, the Q20 flow structures are distinct and, as expected, scale over a larger variety of flows. For the Q80, there are distinct classes, but it is also evident that Classes 3 and 4 are nearly identical in their structures. 
Mean $80 \%$ Exceedence Value per Classification Group

Melt Season (Mar-Jul)

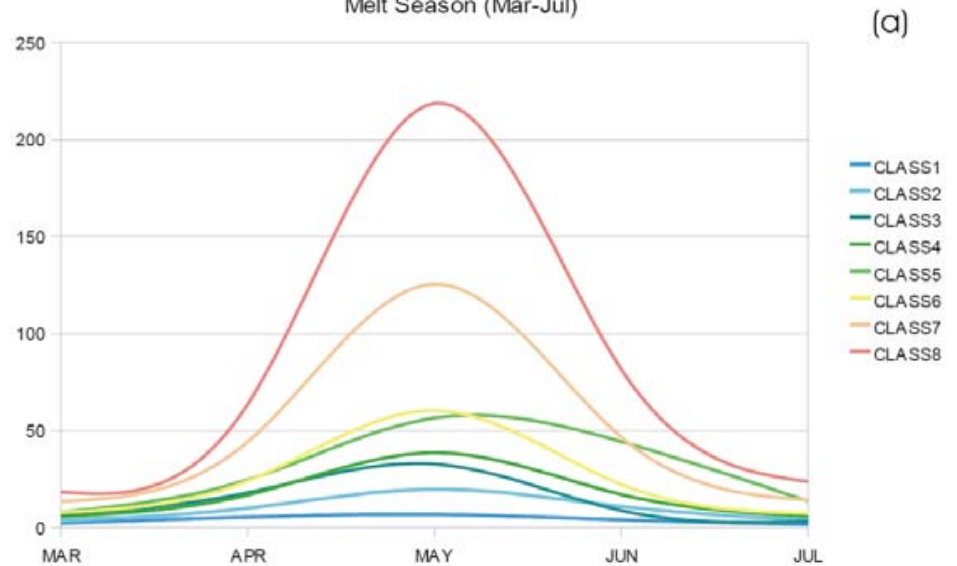

Mean 20\% Exceedence Values per Classification Group

Melt Season (Mar-Jul)

(b)

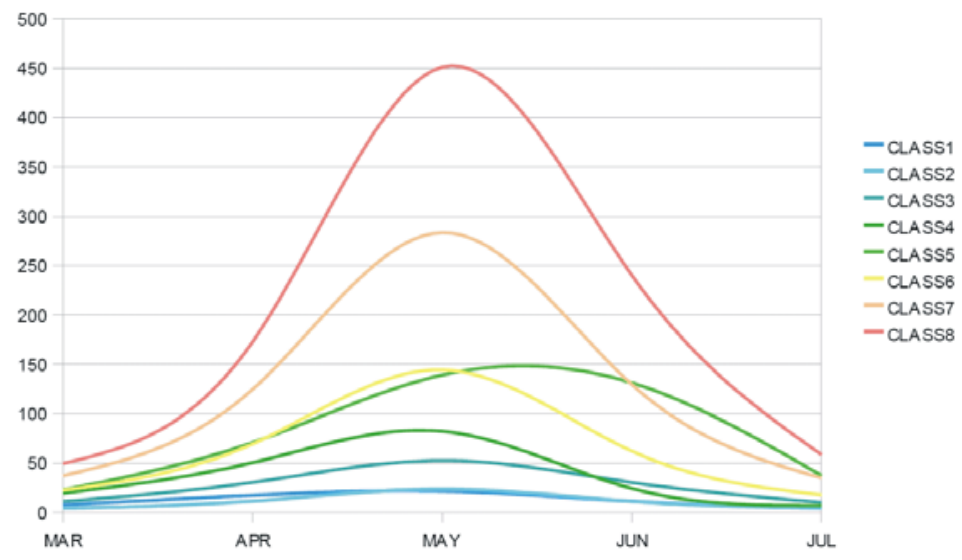

Figure 5.14. Mean values of each SOM cluster per month for (a) Q80 and (b) Q20.

The results of the flow exceedence SOM clustering were mapped back to the 160 spatial containers and a simple similarity comparison was completed between the Q80 and Q20 runs (see Figure 5.15). Again, the classes were sorted such that Class 1 values represent lower flows and Class 8 values represent the highest flows.

Where a change occurred between Q80 class values and Q20 class values, it was attributed into the spatial database and is indicated by a diagonal hatching on the map figure. A total of 15 out of the 160 basins, or $8.5 \%$ of the total area, were dissimilar in their class relationships between Q80 and Q20. Upon further inspection, two of the basins shifted by two classes, from Q80 - Class 6 to Q20 - Class 4, the remainder of the values were shifted $+/$ - one class boundary. 


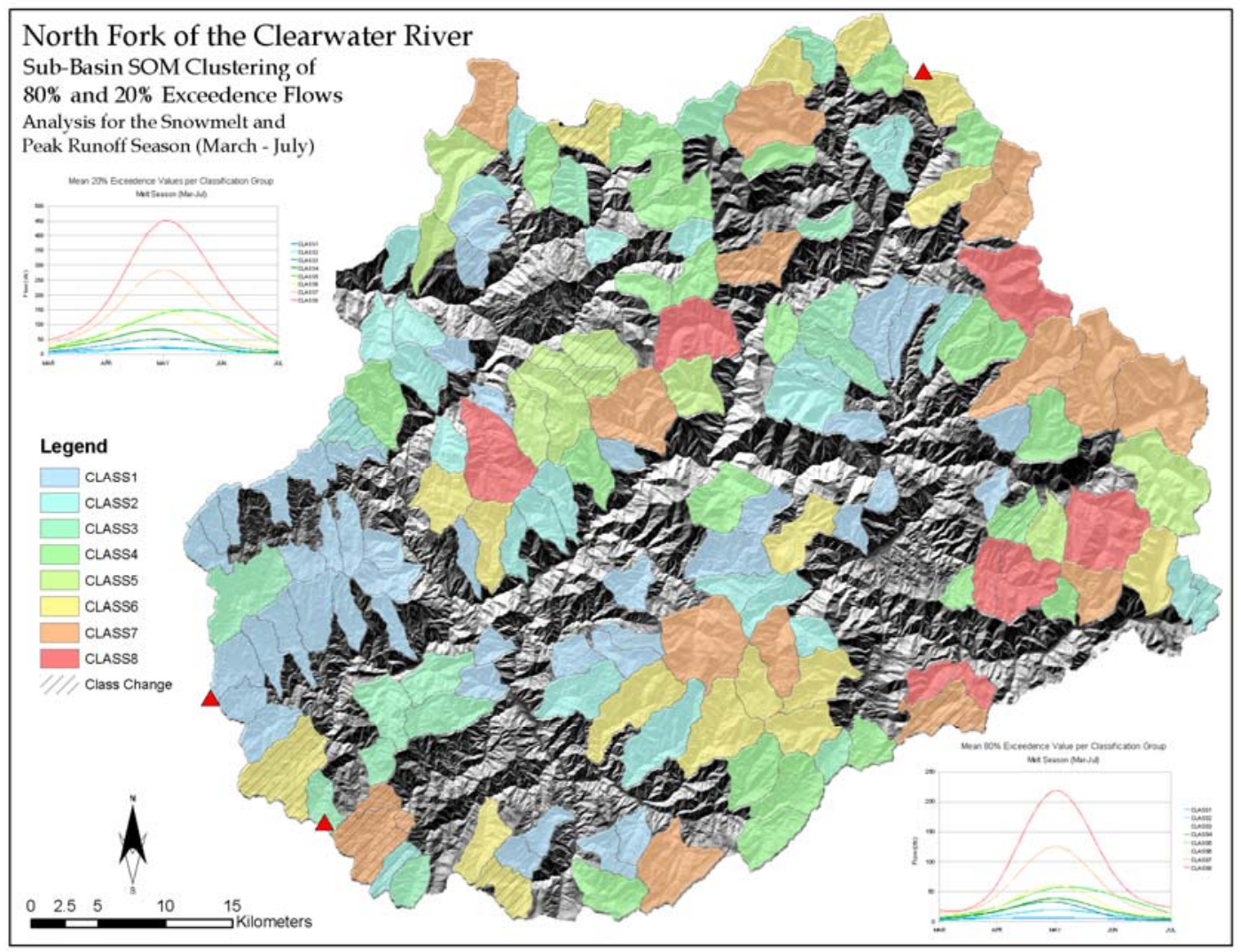

Figure 5.15. Spatial mapping of the Q80 and Q20 SOM cluster results. The classes are sorted based on flow with Class 1 being the lowest and Class 8 the highest.

\subsubsection{Flood Frequency Analysis}

Similar in objective to the work of Hortness and Berenbrock (2001) for flow exceedence values, Berenbrock (2002) provides regression estimations for flood frequencies in both gaged and ungaged basins. For our purpose in this analysis, the "regional regression equations" for ungaged basins are used. Flood frequencies refer to the magnitude and frequency of periods of peak flow and are typically stated in intervals of 2-, 5-, 10-, 25-, 50-, 100-, 200-, and 500-year flood. In other words, these values state the probability of a flood flow of a given magnitude to occur, once in 5 years (5-year flood), once in 100 years (100-year flood), and so on. To determine the flood frequency regression equations, the study area (the state of Idaho) was broken into seven hydrologically similar regions to fine tune the resulting equations. As was the case for the development of regression equations for flow exceedence values, the flood frequency work was built upon the work of others (Kjelstrom and Moffatt, 1981; Quillian and Harenberg, 1982; Thomas et al., 1994; Thomas et al., 1973). A relationship was established between 333 stream gage 
stations and seven basin landscape characteristics, including drainage area ( $D A)$, mean basin elevation $(E)$, percent of area of north-facing slopes greater than $30 \%$ ( NF30), percent of area of slopes greater than 30 degrees ( $S 30$ ), mean annual precipitation $(P)$, percent of forested area $(F)$, and mean basin slope ( $B S)$. The established regression equations for all eight flood frequency periods are provided in Appendix B; however, as an example, the equation for the 100-year flood frequency is provided here:

$$
Q_{100}=5.39 D A^{0.874}(E / 1000)^{-1.13} P^{1.18}
$$

The required parameters needed for the flood frequency analysis on the 160 subbasins had all been derived for the flow exceedence analysis, with the exception of NF 30 (north-facing slopes greater than 30 degrees). This final parameter was implemented into the ALCP, then flood frequency values were calculated using the published regression equations. The results of these data show a consistent pattern, although there is a large range in the flow values and the higher flow basins are clearly fewer in number (see Figure 5.16).

The objective of this analysis is to cluster the flood frequency signal over the nine recurrence periods (i.e., 2-, 5-, 10-, 25-, 50-, 100-, 200-, and 500-year), then map the resulting cluster results back into the spatial database, and subsequently to a map document/display. 


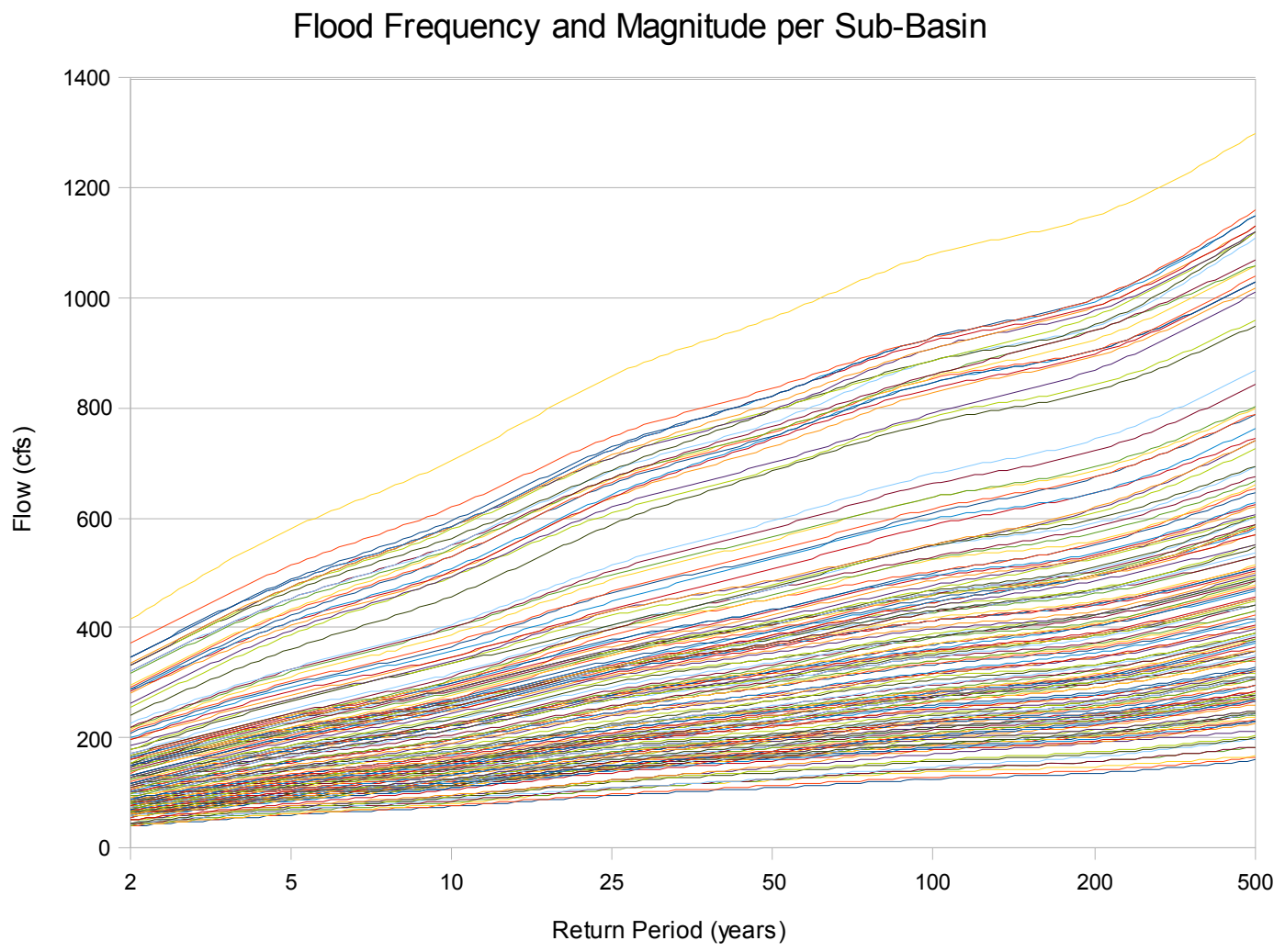

Figure 5.16. Flood frequency values representing both flood magnitude and return period for each of the test basins.

A $10 \times 160$ matrix file was constructed taking on the following form:

$$
I D, Q_{2}, Q_{5}, Q_{10}, Q_{25}, Q_{50}, Q_{100}, Q_{200}, Q_{500}
$$

A 3-D cubic topology SOM with dimensions $2 \times 2 \times 2$ was used to generate eight class boundaries for the flood frequency data. The projected SOM boundaries and output class data were processed to determine mean values for each cluster group. The data were then sorted, and class labels relating to flow values were assigned where Class 1 values are lower flow basins and Class 8 are high flow basins. The mean cluster results, presented in Figure 5.17 reveal a clear distinction between most classes; however Classes 3, 5, and 6 appear to be closely related up to the 50-year return period then begin to modestly separate. For this analysis, it is probable that fewer classes could be used, but it is also important to consider that a 50-75-cfs increase in flow for a small headwater catchment can lead to a significant hydrologic event for that basin. 


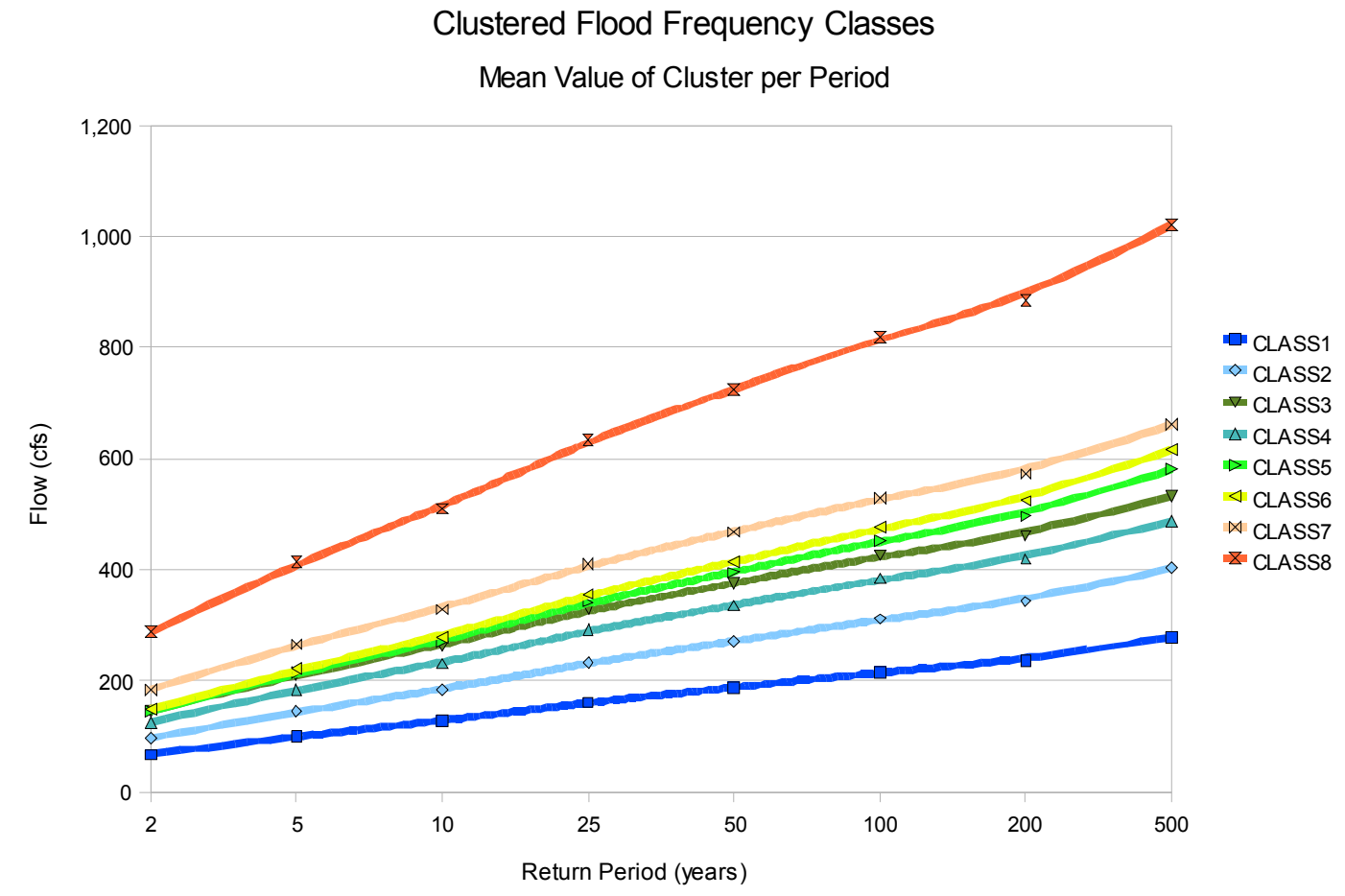

Figure 5.17. Mean cluster values per return period for flood frequency analysis.

Revealing some of the raw data patterns produced from the SOM can help gain a better understanding of the complex signals it processes and groups into classes. In Figure 5.18, the clustered data values for the 5-, 50-, and 500-year flood recurrence intervals are plotted by their cluster groupings, represented by the arbitrarily assigned neuron label (i.e., $\mathrm{x} 0 \mathrm{y} 0 \mathrm{z} 1$ ) on the $\mathrm{x}$-axis and a more easily identifiable line color. Each point along the $x$-axis represents regression-derived flood frequency data for a single sub-basin. While in Figure 5.16 the data appear to take on a very uniform pattern, the results in Figure 5.18 reveal some of the more subtle differences in the regression results. The results, as presented in Figure 5.18, also show the random cluster assignments (i.e., the clusters are not ordered from lowest to highest or by some other mechanism). From the viewpoint of the SOM, its purpose is to find and group common data signals and leave the assignment and processing of those clusters to another method, which after all is the underlying function of any unsupervised classification method.

Further investigation of the flood-frequency data led to the presumption that the subbasin area was the major factor driving the flow magnitude for each recurrence interval. As a test, a linear regression was completed for four of the nine return periods, 2-, 10-, 100-, and 500-year (see Figure 5.19). The flow magnitude values 


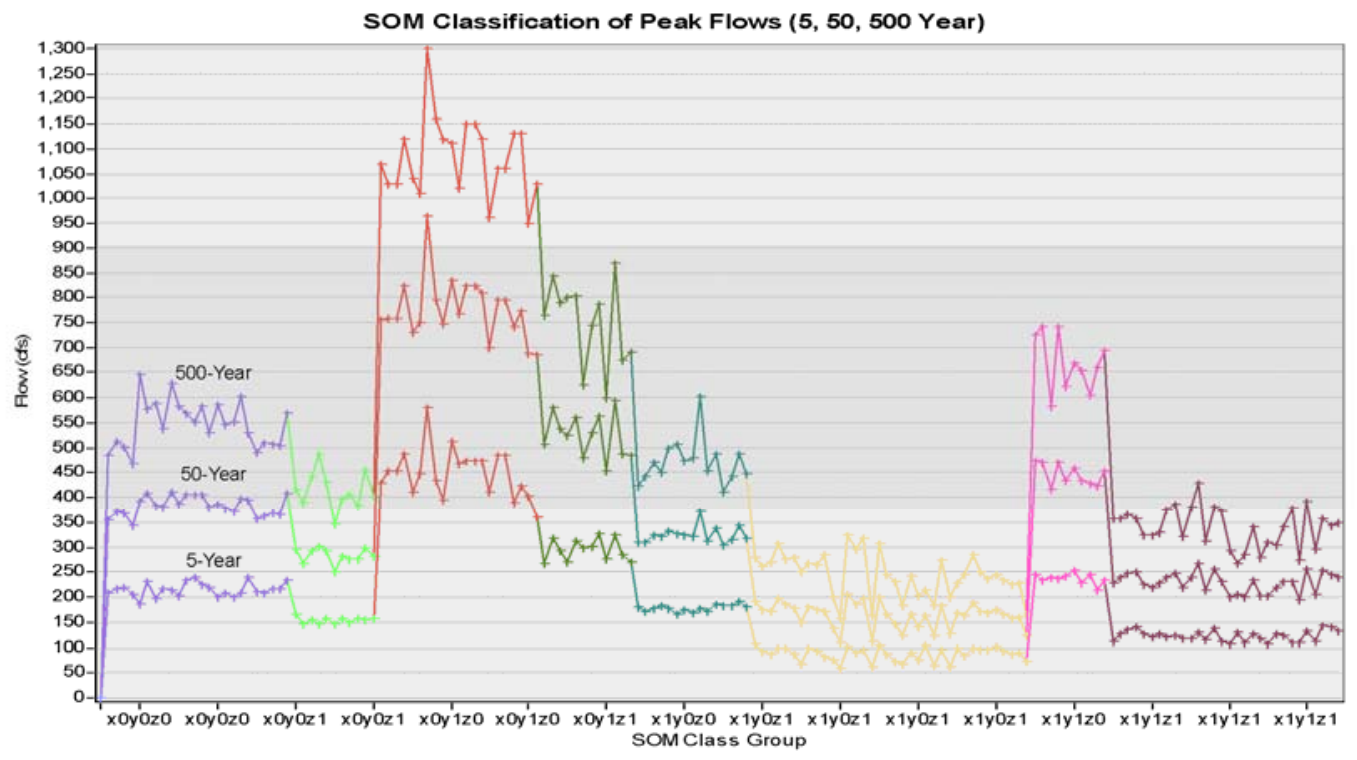

Figure 5.18. SOM classification of flood frequency data for three return intervals over 160 sub-basins.
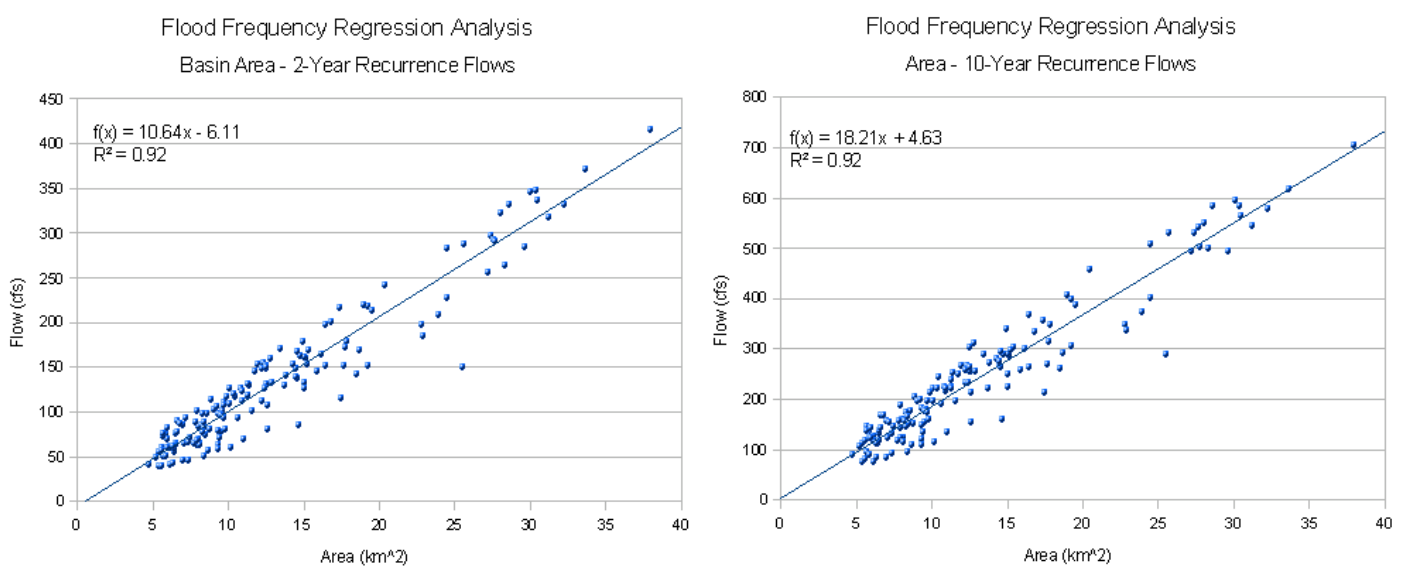

Flood Frequency Regression Analysis

Area - 100-Year Recurrence Flows

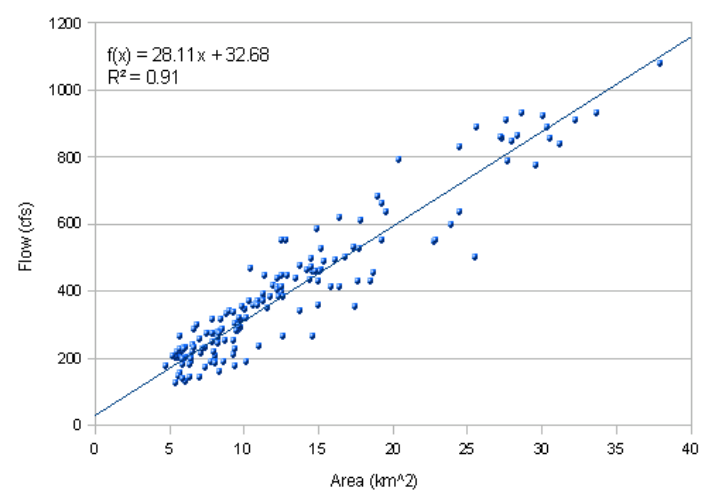

Flood Frequency Regression Analysis

Area - 500-Year Recurrence flow

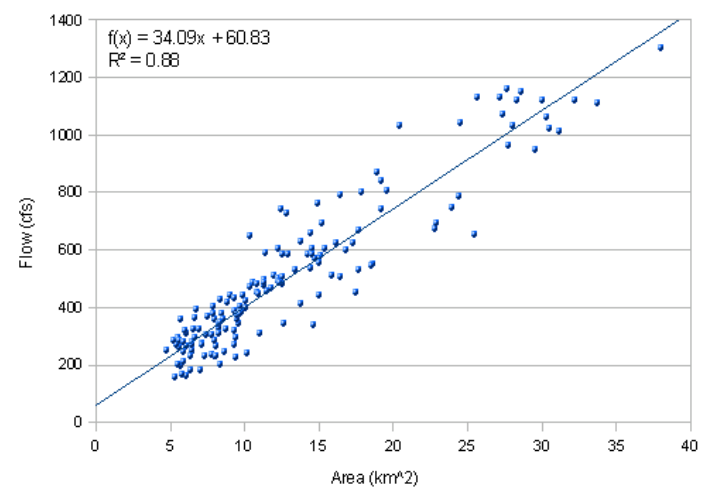

Figure 5.19. Linear regression plots testing the relationship of sub-basin area to flow magnitude for 2-, 10-, 100-, and 500-year return periods.

were tested against sub-basin area and high correlation values, $R^{2}=0.88-0.92$, were found for each recurrence interval. With such a strong correlation, it is perhaps 
unnecessary to perform a SOM analysis on these data, but instead use a simpler method to define the class boundaries. Taking the area and flow magnitude regression data for the 100-year recurrence, the ordered SOM classes, as defined in Figure 5.17, are symbolized on the regression plot to reveal the structure of the class boundaries (see Figure 5.20). Reviewing the plot, it is evident that the class boundaries appear to be reasonably well defined; however, there are some overlaps in the class boundaries. Consider that the SOM analysis was performed using nine periods of flood-frequency data and the representation on Figure 5.20 represents one of the nine periods. Thus, the significance of using the SOM in this example is to give definition to the data signal over the nine periods of time, rather than for a single recurrence interval, which would perhaps be better suited to a different and simpler method of classification. The cluster results of the flood-frequency analysis were mapped back to the 160 spatial containers in the ALCP and are displayed in Figure 5.21.

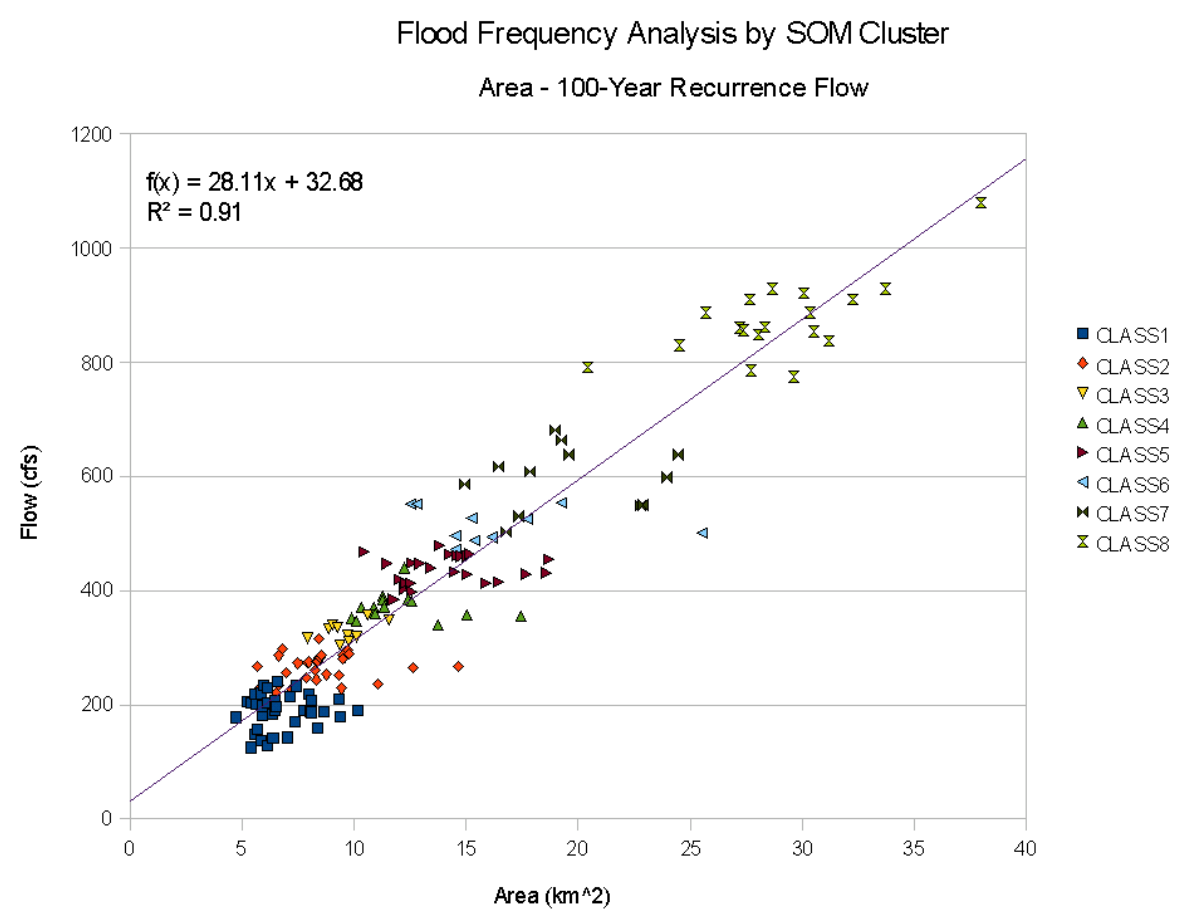

Figure 5.20. 100-year flood frequency regression plot with point members symbolized by their assigned SOM cluster. 


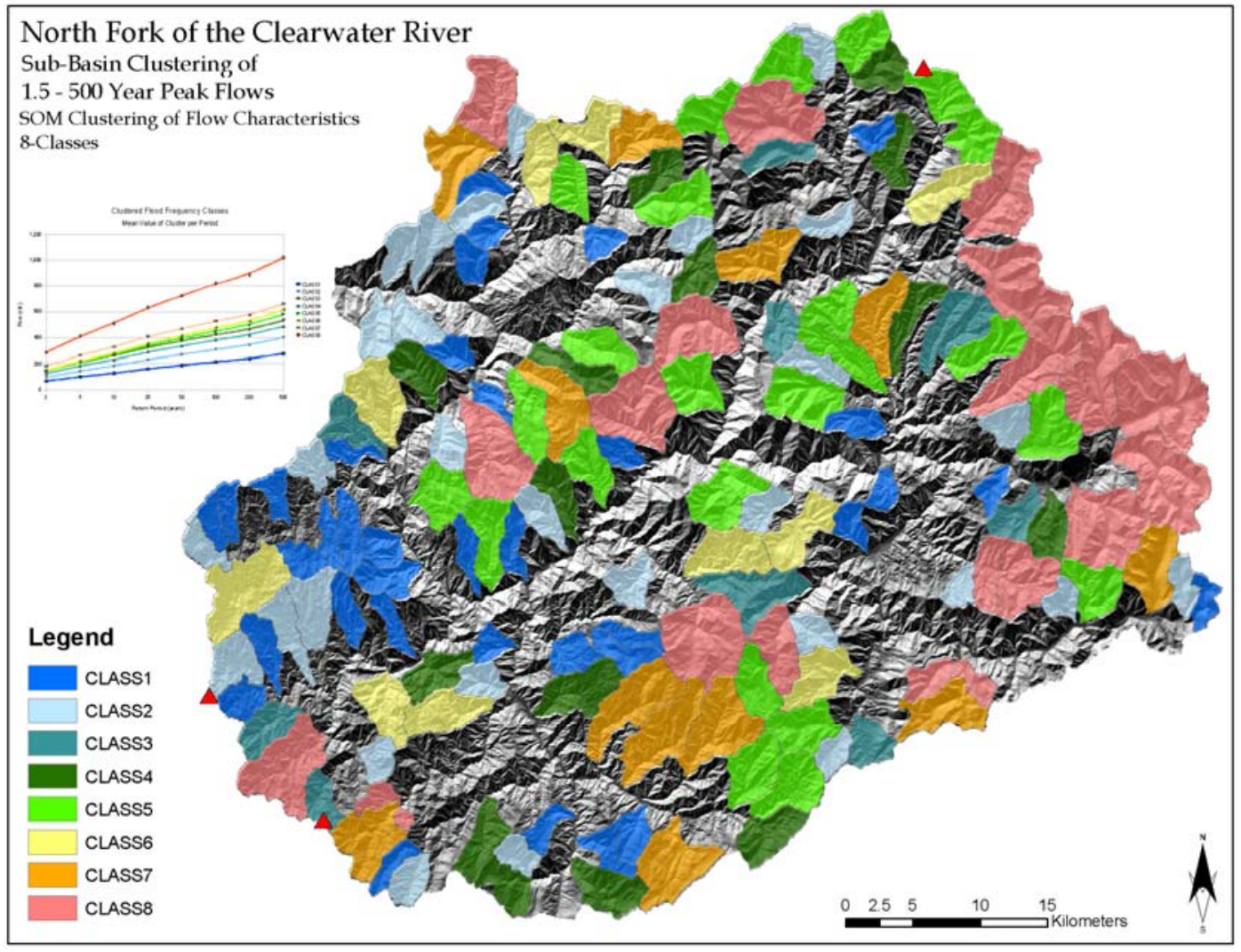

Figure 5.21. Spatial mapping of the nine-period flood-frequency SOM cluster results. The classes are sorted based on mean flow values within each cluster, where Class 1 represents the lowest flows and Class 8 the highest.

\subsubsection{Landscape Characteristics Analysis to Determine Hydrologic Properties}

The primary motive behind the work to develop the ALCP was to derive a method that would allow the classification of complex data representing the natural landscape. Previous sections in this chapter have described and demonstrated promising results from the ALCP, however there is still a fundamental problem in that the classification results can not be validated without a detailed field study to provide truth in the landscape. After much contemplation and research into this issue, the use of published hydrologic multivariate regression equations provides some means of validation and gives definition to the unsupervised landscape classifications.

While the previous two sections (Section 5.3.1 and Section 5.3.2) went into detail concerning the use of the multivariate regression equations used to calculate flow exceedence and flood frequencies, this section focuses on the use of the Q20 as a validation metric for using the ALCP to classify the 160 test sub-basins based purely upon spatially derived landscape data. As was done for the original Q20 analysis, 
the analysis described here focuses on the high streamflow season extending from March through July. The Q20 data were selected over the Q80 and flood-frequency data because of the higher level of statistical confidence reported by Hortness and Berenbrock (2001).

Using the established ALCP spatial database for the North Fork of the Clearwater River, the following 10 landscape metrics for each of the 160 test sub-basins were calculated and used in the classification process: sub-basin area $(A)$, mean elevation $(E)$, maximum elevation $\left(E_{\text {Max }}\right)$, minimum elevation $\left(E_{\text {Min }}\right)$, elevation relief ( $B R$ ), mean slope ( $B S$ ), percent of area with slope greater than 30 degrees (S30), percent of areas of north-facing slopes greater than 30 degrees ( NF30), percent of area with forest cover $(F)$, and mean annual precipitation $(P)$. These values were decided upon after repeated SOM tests with additional values. It should be noted that the source data behind the above-listed landscape metrics comes from a variety of spatial resolutions and their statistical properties within the spatial container are calculated through the ALCP. This helps to demonstrate the ALCP adaptability in using multiple source datasets.

To remain consistent with the previous Q20 analysis, a 2x2x2 cubic topology SOM was initiated using the following $11 \times 160$ input data matrix:

$$
I D, A, E, E_{M a x}, E_{M i n}, B R, B S, S 30, N F 30, F, P
$$

The SOM quantization errors for this analysis initially gave poor results. Repeated iterations of the cluster process were made by adjusting various aspects of the SOM parameters, such as training time, initial neighborhood size, initial learning rate, etc. Upon inspecting the SOM projections overlain in the input data space, it became apparent there was a problem reducing the dimensions of this complex dataset into a small $2 \times 2 \times 2$ SOM structure. Experiments on the SOM dimensions were performed to find the smallest structure to best represent the data. A $5 \times 4 \times 3$ structure, representing a total of 60 classes, was determined to meet this need by finding the balance point between lowest quantization errors and number of classes. The final projected SOM structure and data space are presented in Figure 5.22. 

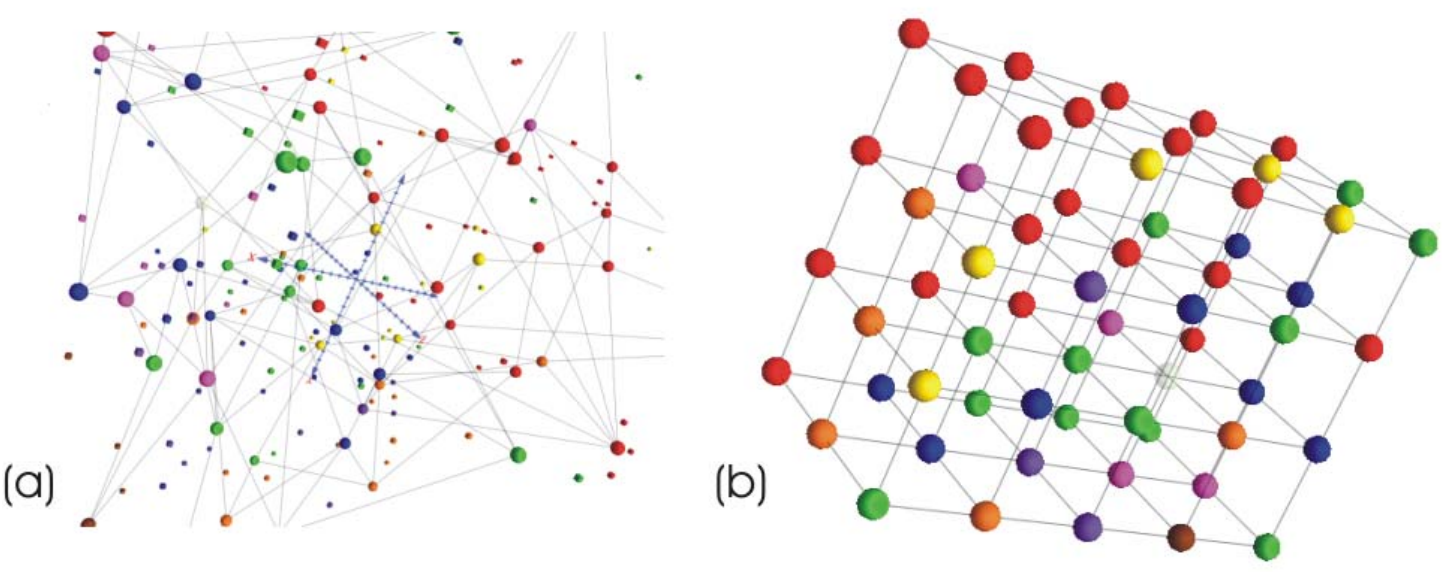

Figure 5.22. The input data space (colored cubes) and final projected neurons (spheres) in (a) natural projection space and (b) structured representation. The final SOM structures represent the clustering of 10 landscape metrics. Note that one neuron, best viewed in (b), was not used, indicating a sufficient number of neurons used.

To construct a similarity measure against the Q20 analysis, it was necessary to relate the 60 classes of landscape metric data to the eight classes of the Q20 data. The SOM processing allows a data label assignment to be carried through with the codebook vectors. In this case, the class labels from the Q20 analysis were joined with the remaining landscape metric data. In the final clustered dataset, a manual process of taking the determined clusters from the landscape SOM and determining the associated and dominant Q20 class was performed. For example, in the following case (see Table 5.4), one landscape neuron has clustered four data points. Three of the four data points are associated with Class 2 in the Q20 analysis, and the remaining data point is associated with the Class 3 group. In this case, Class 2 is assigned as the dominant class for the neuron, and a field indicating the degree of change in class boundaries is indicated.

Table 5.4. An example demonstrating the dominant class and degree of class difference for data assigned to a given neuron.

\begin{tabular}{|l|l|l|l|}
\hline Landscape Neuron & Q20 Class & Dominant Class & Class Difference \\
\hline X0y1z1 & Class 2 & Class 2 & 0 \\
\hline X0y1z1 & Class 2 & Class 2 & 0 \\
\hline X0y1z1 & Class 3 & Class 2 & 1 \\
\hline X0y1z1 & Class 2 & Class2 & 0 \\
\hline
\end{tabular}


In many cases, this was a straightforward process where one data point in the group was different. Other cases included mapping a one-to-one relationship, because there was only one neuron assigned to a given data point (i.e., sub-basin). In cases where there was a tie in determining the dominant class (i.e., two values indicated Class 3, and two values indicated Class 1), the existing Q20 class value was passed through as the dominant class, and a class difference was reported as the difference between all classes involved in the tie (i.e., the difference between Class 3 and Class 1 is 2 , thus all values involved in the tie are assigned a class difference of 2 ). Once this process was completed, a review of the results, in terms of the percent of similarity between the landscape class value and the Q20 class value was performed. Further, for classes that weren't similar, an additional analysis of the degree of difference was performed. The results, shown in Figure 5.23, indicate a similarity measure of $69.8 \%$, or 112 of the 160 basins, between the landscape classes and the Q20 classes. Of the $30.2 \%$ of the basins that had a measure of dissimilarity, $39.6 \%$, or 19 basins, had a difference of 1 class boundary, and $22.9 \%$, or 11 basins had a difference of 2 class boundaries. The final results were mapped back into the spatial database and are presented in Figure 5.24. Commonly shared basins between the Q20 and landscape clusters are mapped with successive colors (Classes 1-8), which are indicative of the amount of streamflow, similar to what has been presented previously. The basins evaluated as being dissimilar are colorcoded with an overlain hatch marking to indicate the divergence between the two cluster operations in addition to the degree to which they are dissimilar.

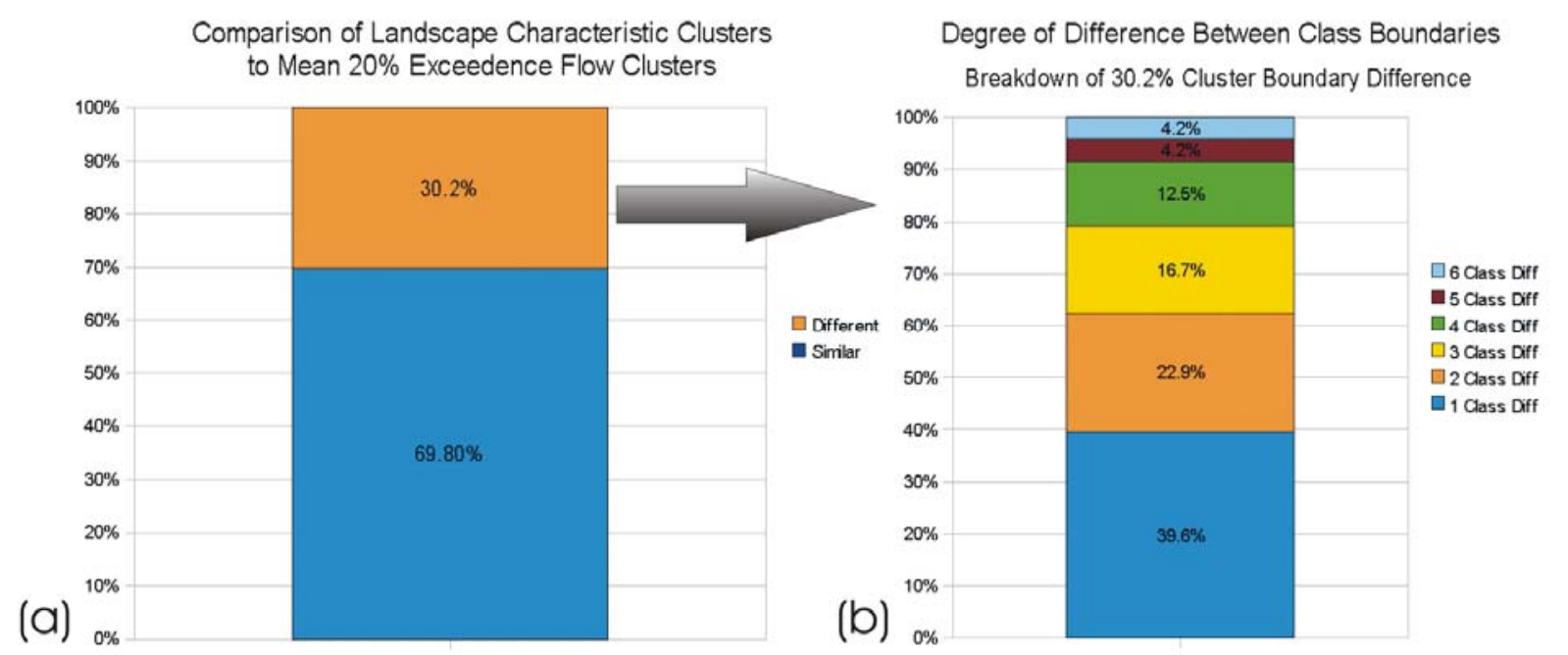

Figure 5.23. Bar graphs indicating (a) the overall similarity in the landscape and Q20 class boundaries, and (b) the degree of class change for those basins that were identified as being dissimilar. 


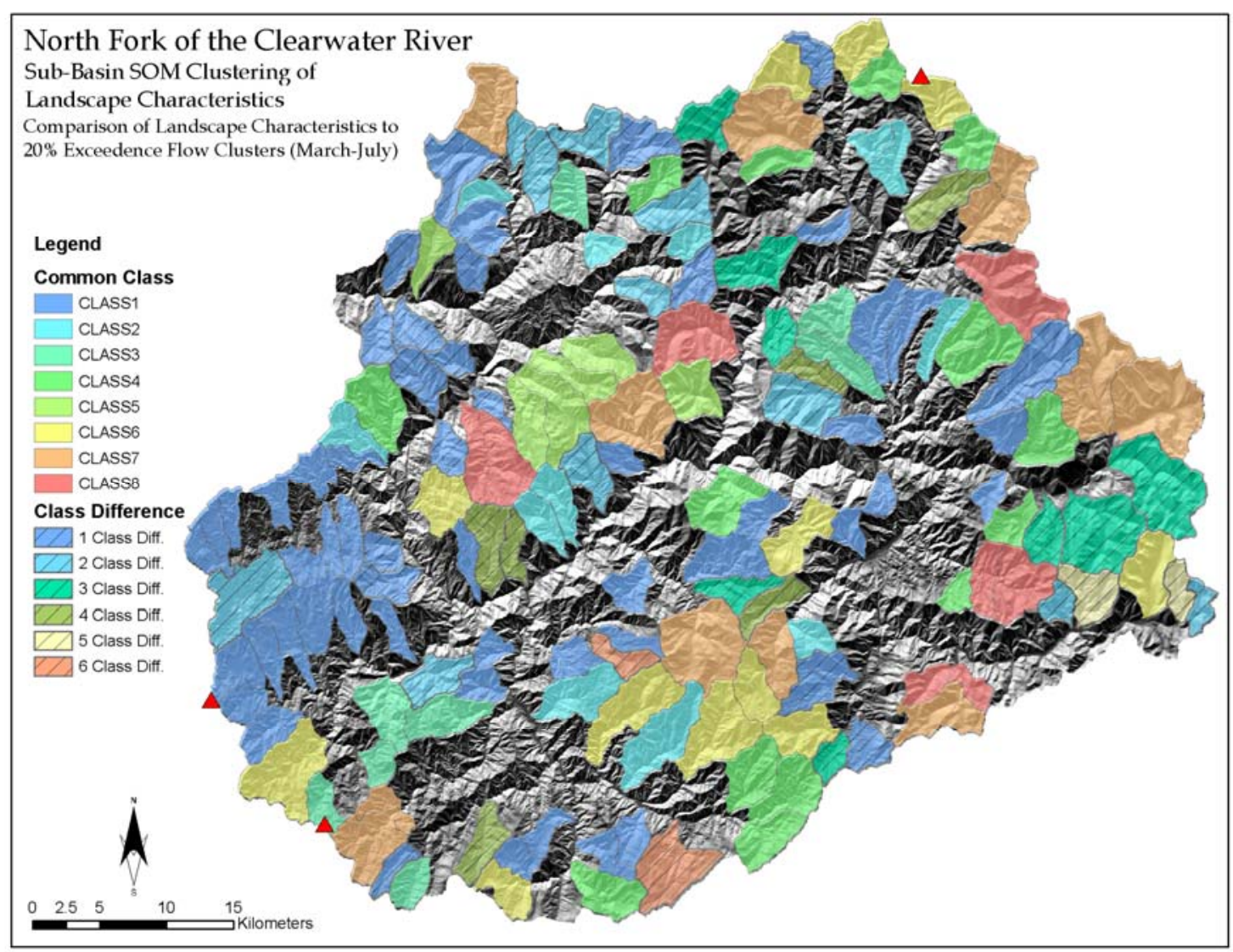

Figure 5.24. Similarity index map showing likeness and difference between two independent cluster analyses, using 1) spatial landscape metrics, and 2) Q20 regression equations and landscape metric data to feed the regression equations.

While the reported results show a reasonable degree of success, a number of factors may be contributing to the reported outcome. First, the reduction of the 60 landscape-determined classes to the eight Q20 classes, as discussed previously, involved a procedure for determining dominant classes and a method for dealing with cases where there were ties. For this analysis, there were 38 ties, for which a conservative approach was taken where each member of the tie group was counted as dissimilar. Another approach would have been to randomly select one of the cluster groups to break the tie, consider this the dominant class, then only count the remaining cluster members as being dissimilar. Using this method would have increased the number of similar basins by $19(11.88 \%)$, bringing the overall percentage of similar basins to $81.7 \%$. 
Upon further research into the establishment of the USGS multivariate regression equations for Q20 and Q80, it was determined that the spatial datasets used to develop these equations are somewhat different than what was used in this analysis (see Table 5.5). This difference may have contributed to the dissimilar nature of some of the test basins. The dataset difference that is likely to have the largest impact is the source data for the mean annual precipitation. The difference in spatial resolution (i.e., $4 \mathrm{~km}$ vs. $800 \mathrm{~m}$ ) is likely to have some impact, especially considering the size of the sub-basins being used in the analysis. There also have been many PRISM model updates and dataset fixes in the time period spanning the release of each of the datasets.

Table 5.5. Source spatial data the USGS used to support and develop multivariate regression equations (left), and the data source used in the landscape analysis test (right).

\begin{tabular}{|l|l|}
\hline \multicolumn{1}{|c|}{ USGS Source Data } & \multicolumn{1}{c|}{ ALCP Source Data } \\
\hline USGS 30 m DEM & USGS 10 m DEM \\
\hline $\begin{array}{l}\text { National Elevation Dataset Hydrologic } \\
\text { Derivatives (NED-H) }\end{array}$ & $\begin{array}{l}\text { Hydrologic data derivatives calculated by } \\
\text { ALCP using 10 m DEM data as a source. }\end{array}$ \\
\hline $\begin{array}{l}\text { 1998 National Land Cover Dataset } \\
\text { (NLCD) }\end{array}$ & $\begin{array}{l}2001 \text { National Land Cover Dataset } \\
(\text { NLCD) }\end{array}$ \\
\hline $\begin{array}{l}\text { Annual precipitation for the western } \\
\text { United States (1961-1990), } 4 \mathrm{~km} \\
\text { resolution (PRISM). }\end{array}$ & $\begin{array}{l}\text { Annual precipitation for the western } \\
\text { United States (1971-2000), 800 m } \\
\text { resolution (PRISM). }\end{array}$ \\
\hline
\end{tabular}

The Q20 and Q80 regression equations also incorporated 33 years of local stream gage data; however, because this analysis was conducted solely on small ungaged basins, it was not possible or of benefit to incorporate these data into the SOM analysis.

As discussed earlier in this document, it is recognized that SOMs can have some difficulty with outlier data, particularly when the neuron structure isn't large enough to represent the data point extremes. The SOM will try to incorporate these outliers, assigning them to the closest neuron when the SOM completes its data projection. As a result, these outlier data can be lumped into a cluster group that is not necessarily appropriate.

Finally, it is possible that a better classification of the Q20 data may be obtained by experimenting with different combinations of the available ALCP data elements by 
increasing and decreasing the number of elements and determining the sensitivity or influence of each dataset. Additionally, experiments with regards to weighting the input data where a higher weight is assigned to those data elements which tend to have a stronger correlation to the Q20 values (i.e., basin area) and a lower weighting value to those data elements with a weaker correlation could lead to improved results. Ideally, a future validation process may be able to use instrument data, a calibrated hydrological model, or a scenario such as that which was discussed in Section 5.1 (Multi-Spectral Classification) where detailed on-the-ground field data were collected. 


\subsection{Conclusions}

This research has documented the development of a procedure that addresses and demonstrates three research objectives defined in the introductory chapter of this thesis. This concluding chapter will specifically address each of the research objectives in terms of design and the demonstrated application of the ALCP. Further, a discussion on the limitations of the ALCP and future development considerations are presented.

\subsection{Conclusion of Research Objectives}

The foremost problem being addressed in this research is whether or not it is possible or feasible to convey the knowledge in one landscape domain to other domain areas that exhibit similar characteristics, but do not have the same level of detail in the data. This research question is rooted in the idea of heterogeneity which can be viewed in many different ways. It is well recognized that the natural landscape possesses a complex interaction of biotic and abiotic processes which define the form and function of the landscape. Depending upon the research question posed to address a certain problem, or better understand a process, and further, depending upon the discipline working to answer the question, the idea of heterogeneity will be different. For example, the hydrologist may only wish to evaluate the diversity in flow patterns or variations in water quality, whereas the ecologist may look at differences in biotic diversity or connectivity and patch densities. The development and application of the ALCP addresses heterogeneity, including not only the variations found in the natural landscape, but also with data collected and used to represent and define the landscape. Heterogeneity in data is found in many forms including diverse scales and resolution of source data, differences in data collection methods and/or standards, and changes in political or administrative boundaries. A combination of the variations in the natural landscape attributes and diversity in source data leads to complex heterogeneity in the landscape for which the ALCP has been demonstrated as a promising procedure capable of reducing this complexity into manageable homogenous units. As was demonstrated in application of the 30-year annual mean climatology, flow exceedence, flood frequency, and most especially in the use of landscape characteristics to determine hydrologic properties, the ALCP's spatial container works to bring various data sources together under a common spatial unit which can 
then be used for pattern evaluation and data reduction of the combined data attributes for the purpose of generating a manageable and useful data classification. While not a perfect demonstration of conveying knowledge from one domain to another, the landscape characteristics analysis to determine hydrologic properties (see Section 5.3.3) was used to demonstrate the possibility of using various spatial data attributes to predict Q20 flow exceedence values in the landscape and revealed promising results for inferring and propagating knowledge across the landscape. This type of analysis would be further benefited by having in situ data or physical model simulations for a select number of the analysis basins, ultimately providing a level of confidence in the classification capabilities and furthering the applicability of conducting an unsupervised classification to find similarities in the landscape and using these results to propagate knowledge to other areas in the spatial domain. These types of support data would ideally demonstrate real-world conditions where typically it is only feasible to collect data in a limited number of locations. Provided the data collection locations are unique, as was demonstrated with the 30 -year mean annual climatology analysis (see Section 5.2), it is then conceivable to propagate this data throughout the landscape. In the future, it is desirable that the ALCP can be further tested using spatial data patterns to define monitoring sites, collect field data in the uniquely classified areas, and running tests, including blind tests, to gain further confidence in the procedure.

Improving the understanding and linkages between ANNs and geoinformatics presented a fundamental research question which is encompassed in the whole body of this research. It is well-recognized from the work of others referenced throughout this thesis, as well as through the presented research, that ANNs provide a unique capability to evaluate data in ways not possible with other classification methods, particularly when the datasets are large, complex, and non-linear. Literature reviews conducted at the beginning of this research seeking the use of ANNs in GISc revealed work had been done in this area, but was rather limited, and in most cases, the GIS and the ANN were loosely coupled, making the process flow difficult and inefficient. While there are many different types of ANNs, as discussed in Chapter 3 , only the SOM was chosen for this research, however the framework of the ALCP provides the fundamental building blocks for incorporating other ANNs into the procedure. The spatial container provides an important GIS / ANN link as it provides the means for carrying various spatial data into the ANN for processing then bringing the results back into the GIS for visualization and further analysis. While it is clear more work is needed to further refine and automate the process flow in the ALCP, 
nonetheless, this research and particularly the demonstration applications presented in Chapter 5, have illustrated the reality of linking the powerful capabilities of both GIS and ANNs. The coupling of geoinformatics and SOM technologies for reducing large amounts of diverse, complex, non-linear, and high-dimensional data into a simpler classified form provide a capability where resulting unsupervised classified data are available for query and exploration within a visual GIS environment and can further be used to infer and/or predict landscape processes by discovering spatial patterns in the data.

The design and development of the ALCP was specifically intended handle diverse and complex data in a spatial environment and provides an alternative to traditionally used classification methods in GIS. The review of currently and commonly used landscape classification procedures in Chapter 2 led to the understanding of their capabilities and limitations, furthering the need to investigate a more powerful and adaptable classification procedure. This process involved gaining a perspective of the power and advantages of using ANNs for classification, the capabilities and limitations of SOMs, the development of the ALCP, and the testing of the ALCP on several problem sets. The final analysis (see Section 5.3.3) demonstrated the ability to use spatial characteristics in the landscapes to determine and convey specific hydrologic properties in the landscape. While a comparison study using different classification methods was not included in this research largely because this has been done elsewhere (see Chapter 2 Introduction), the use of the SOM as the core classifier in the ALCP has shown favorable results in all of the demonstration applications. It is clear that the diverse and adaptable capabilities of the ALCP allows an intelligent use of available data for purposes of gaining a holistic perspective of many landscape aspects, understanding differences and similarities in the landscape by evaluating single or multiple elements, and relating known information to other areas within the study domain that exhibit similar qualities. The ALCP can be used for applications requiring prediction, planning, monitoring, what-if scenarios, and understanding the impacts of various elements in the landscape.

\subsection{Limitations of the ALCP}

While the procedures developed in this research appear promising for many application areas, there are limitations that need to be considered. First, the number of neurons selected for the SOM will have an impact in several ways. First, in the 
case where a "small SOM structure", or a small number of total neurons, is used, caution must be exercised to ensure the data is being properly represented and the neurons are effectively being projected across the data space. Of particular interest are outliers in the data which can represent extreme events, and thus important to identify as these commonly shape landscape processes. If using a small SOM structure, the outlier data will likely be associated with the nearest neuron representing a larger group of data, and the characteristics of the outlier information will be lost. Some literature suggests using one neuron for every input record, so conceivably, if every data vector being presented to the SOM has a large difference from one another, then every neuron will be assigned uniquely. This suggestion basically allows the natural data clusters to be found, which will more than likely leave a significant number of neurons unassigned to any data because there will be similarities found within the data record. From the perspective of unsupervised classification, this can lead to some issues because instead of having 25 classes to provide definitions for, you may have 125 natural data clusters which, depending upon the scope of the study, may not be reasonable and perhaps provides too much detail. Nonetheless, it is recommended to start the SOM process with a large number of neurons so the natural data clusters can be evaluated. Unfortunately, there are no hard logic rules for determining the correct number of neurons to use, and finding the ideal balance between the number of neurons required to represent and properly project the data with reasonable SOM quantization errors, and the appropriate number of class divisions for the study, is a trial-and-error process.

A second limitation is that in order to best use the ALCP for the propagation of information across the landscape, a large number of diverse samples is required. Fundamentally, this concept is reasonable in that the system needs to see and understand different data in order to classify it as a unique class. In other words, if all the data you have presented to the ALCP represents mountainous and forested landscapes, it has no concept of what a desert is, and thus if desert data is presented to a trained SOM, the SOM will assign the desert data to the closest pattern match according to what it knows. This idea leads into another limitation of the ALCP, which is "free form analysis". In other words, the ALCP in its current form contains no error checking or rules to define what is appropriate and inappropriate use of data. Thus, the burden is placed upon the user to understand what variables are important to present to the ALCP and what the classification results mean. It is always recommended to evaluate the results against an independent means (i.e., field data, supervised classification, physical model, etc.) which provides a level of confidence 
in the classification. An independent classification of data can be completed for a small but representative area to initially setup up a new application and allows for the determination of the right combination of data variables and number of neurons to use in the process.

Another potential limitation of the ALCP is the implicit use of an unsupervised classification, i.e., the input data patterns are classified, but it is up to the user to define the significance of those patterns. This requires analysis and post-processing (i.e., attributing) of the resulting data. Early design concepts of the ALCP considered using a hybrid ANN approach where the SOM was used to classify the data patterns, then these patterns are passed onto a supervised ANN model, such as a Multi-Layer Perceptron, to provide the attribute definition of the data cluster (see Figure 6.1). The development of this procedure is still viable, however, it appears outside of the scope of the current research.

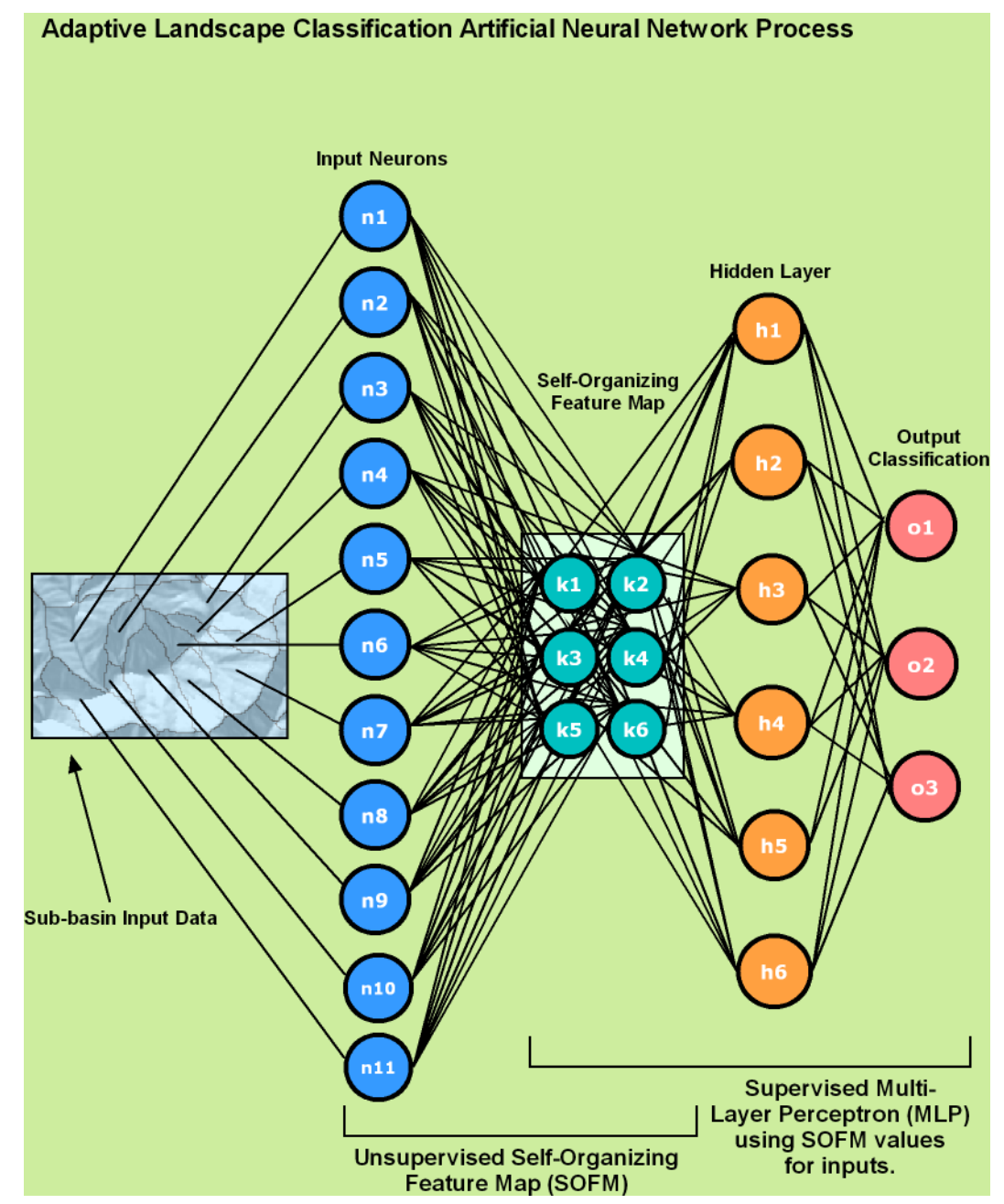

Figure 6.1. A design concept for a hybrid ANN model combining the unsupervised SOM classification with a supervised ANN such as the Multi-Layer Perceptron, resulting in a supervised classification of spatial data. 
Lastly, the majority of the program code written for the ALCP was completed using Arc/INFO AML, which still remains a supported language, but along with Arc/INFO Workstation software, has not been further developed since the release of the newer ArcGIS platform. This potential limitation is acknowledged, however it was determined early in the research process that ArcGIS would not be able to complete all the required tasks without a massive programming effort to recreate functions that already existed in Arc/INFO workstation. Ideally, in the future, the ALCP will be migrated to an open-source GIS framework that is capable of functioning on multiple platforms (i.e., Linux, UNIX, MacOS X, Windows) and will match the flexible capability of the underlying Java-based SOMMER code which can also run on multiple platforms.

\subsection{Future Development Considerations}

Future development work on the ALCP is noted here to help define new possibilities and to acknowledge the thought process for this research, but to also recognize the need to keep defined boundaries on the current research. Two future development considerations were already brought forth in the previous section involving the use of a hybrid ANN for the purpose of developing supervised, rather than unsupervised classifications, and the future need to bring the ALCP into an open-source GIS framework. The remaining sections provide additional considerations for future development.

\section{Optimization Parameters}

As was discussed in Section 6.2, a current limitation of the ALCP is the trial-and-error process required to determine the ideal number of neurons to use in a classification process. The number of neurons to use not only depends on data space and needs of the research scope, but also many parameter values used to drive the actual SOM process. Additional work in this area should be conducted to develop automated calibration routines which utilize the available datasets with a range of possible SOM parameter values. The automated procedure would work to optimize the SOM parameter values in order to find the delicate balance point between number of neurons to properly represent the data space and the ideal number of class boundaries for the problem set. 


\section{Data Sensitivity Analysis}

To further the concept of developing automated optimization parameters, another future capability is the automated evaluation of data sensitivity. By evaluating the impact, or sensitivity, of any given data variable used in the ALCP, the user can gain a sense for what elements in the landscape appear to have a significant effect on the overall classification process. This automated process would entail a complex process of mixing, dropping, and recombining different data variables along with automated adjustments to the SOM parameter values as discussed in the previous section. Such a process may require an outside evolutionary computing processor such as Particle Swarm Optimization to efficiently determine ideal parameters, rather than using a computationally-heavy trial-and-error process.

\section{$\underline{\text { SOM Attribute Weighting }}$}

A hypothesis is presented for future consideration which involves the testing of "SOM Attribute Weighting". The basic hypothesis investigates the possibility of expanding or contracting a data signal with more or less data surrounding a particular data theme for the purpose of adjusting its weight in the overall classification. For example, as presented in Figure 6.2, the vegetation theme is determined to be an important factor, and thus needs to be weighted heavier, in the classification process and as a result includes 17 different variables creating a larger portion of the data signal. Conversely, the aspect theme is determined less important and thus occupies a smaller space in the data signal with just two variables. As it currently exists, the data elements presented to the SOM all have equal weight and this future capability development, provided the hypothesis stands true, would allow the specification of weights to indicate the importance of different data themes. 


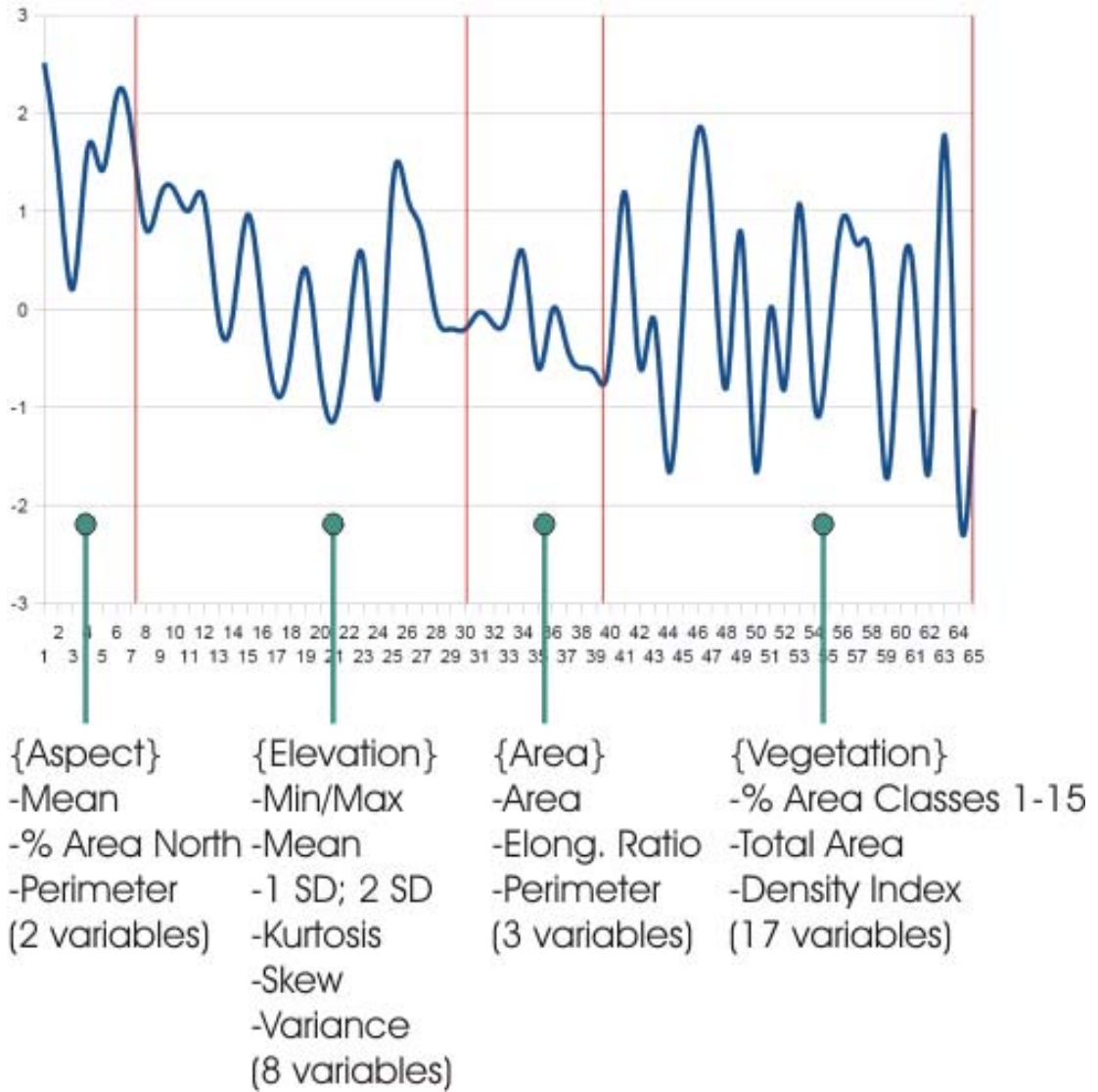

Figure 6.2. The concept of SOM Attribute Weighting is presented as a potential method for assigning data theme weights is the classification process.

\section{$\underline{\text { SOM Trajectories }}$}

Additional future work for the ALCP could involve the development of trajectories on the SOM-classified data for the specific purpose of better analyzing spatial data patterns over time. The trajectories would keep track of where and how classified data are moving over space. This type of capability would facilitate the understanding of diurnal, seasonal, inter-annual, inter-decadal cycles of change and may become important for studying climate change responses of specific elements in the landscape. The process would require additional database elements to keep track of the classification history and a processor to interpret and visually convey the direction of movement.

\section{Comparison Tests}

Prior to any of the aforementioned capabilities development, the ALCP needs further testing against independently-derived classification results which may involve the use of field-collected data, supervised classifications, or various physically based model 
results that simulate various land and surface components of the landscape. These tests are necessary to gain a level of confidence for various applications areas and to further the understanding of the capabilities and adaptability of the ALCP. A realworld exercise of using field-collected or other detailed data for limited areas and propagating this knowledge across the landscape is essential for moving the ALCP from a theoretical state to a state of applied and exercised for the benefit of helping to solve problems or to discover new patterns and relationships in existing data. 


\subsection{References}

Aleksander, I and H Morton. 1990. An Introduction to Neural Computing. Chapman and Hall, London.

Atkinson, PM and ARL Tatnall. 1997. Neural networks in remote sensing Introduction. International Journal of Remote Sensing. 18(4):699-709.

Bacao, F, V Lobo and M Painho. 2004. Geo-self-organizing map (Geo-SOM) for building and exploring homogeneous regions, Geographic Information Science, Proceedings. Lecture Notes in Computer Science, pp. 22-37.

Bacao, F, V Lobo and M Painho. 2005a. The self-organizing map, the Geo-SOM, and relevant variants for geosciences. Computers \& Geosciences, 31(2):155-163.

Bacao, F, V Lobo, M Painho. 2005b. Self-organizing maps as substitutes for k-means clustering, Computational Science - ICCS 2005, Pt 3. Lecture Notes in Computer Science, pp. 476-483.

Bailey, RG 1995. Ecoregions of the United States. United States Department of Agriculture, Forest Service. Accessed April 11, 2006 at http://www.fs.fed.us/land/ecosysmgmt/ecoreg1 home.html (last updated 17 August 2001).

Bailey, RG. 2004. Identifying ecoregion boundaries. Environmental Management, 34 (Suppl 1):S14-S26.

Ball, GH and DJ Hall. 1965. ISODATA, A Novel Method of Data Analysis and Pattern Classification. Stanford Research Institute, Menlo Park, California., pp. 79.

Bathgate, JD and LA Durham. 2003. A geographic information systems based landscape classification model to enhance soil survey: A southern Illinois case study. Journal of Soil and Water Conservation, 58(3):119-127.

Berenbrock, C. 2002. Estimating the Magnitude of Peak Flows at Selected Recurrence Intervals for Streams in Idaho. U.S. Geological Survey Water Resources-Investigations Report 02-4170, United States Geological Survey, Boise, Idaho.

Bishop, CM. 1996. Neural Networks for Pattern Recognition. Oxford University Press, New York, pp. 504.

Bolliger, J. 2005. Simulating complex landscapes with a generic model: Sensitivity to qualitative and quantitative classifications. Ecological Complexity, 2(2):131149.

Bolliger, J, and DJ Mladenoff. 2005. Quantifying spatial classification uncertainties of the historical Wisconsin landscape (USA). Ecography (28):141-156. 
Bolliger, J, LA Schulte, SN Burrows, TA Sickley, and DJ Mladenoff. 2004. Assessing Ecological Restoration Potentials of Wisconsin (U.S.A.) Using Historical Landscape Reconstructions. Restoration Ecology 12(1):124-142.

Brown, DG, DP Lusch, KA Duda. 1998. Supervised Classification of Types of Glaciated Landscapes Using Digital Elevation Data. Geomorphology 21(3-5): 233-250.

Bryan, BA. 2003. Physical Environmental Modeling, Visualization and Query for Supporting Landscape Planning Decisions. Landscape and Urban Planning 65: 237-59.

Bryan, BA. 2006. Synergistic techniques for better understanding and classifying the environmental structure of landscapes. Environmental Management 37(1): 126-140.

Burrough, PA, JP Wilson, PFM van Gaans, and AJ Hansen. 2001. Fuzzy k-means classification of topo-climate data as an aid to forest mapping in the greater Yellowstone area, USA. Landscape Ecology 16(6):523-546.

Burt, TP and DP Butcher. 1986. Topographic Controls of Soil Moisture Distributions. European Journal of Soil Science 36(3):469-486.

Caratti, JF, JA Nesser, and CL Maynard. 2004. Watershed classification using canonical correspondence analysis and clustering techniques: A cautionary note. Journal of the American Water Resources Association, 40(5):12571268.

Carroll, ML, CM DiMiceli, RA Sohlberg, and JRG Townshend. 2004. 250m MODIS Normalized Difference Vegetation Index, 2001-2006. University of Maryland, College Park, Maryland. Accessed April 11, 2006 at http://glcf.umiacs.umd.edu/data/ndvi/ (undated webpage).

Catani, F N Casagli, L Ermini, G Righini, and G Menduni. 2005. Landslide hazard and risk mapping at catchment scale 2005 Arno River basin. Landslides 2(4): 329-342.

Chen, SK, P Mangiameli, and D West. 1995. The Comparative Ability of Selforganizing Neural Networks to Define Cluster Structure. Omega, International Journal of Management Science, 23(3):271-279.

Chon, TS, YS Park, KH Moon, and EY Cha. 1996. Patternizing communities by using an artificial neural network. Ecological Modelling 90(1):69-78.

Civco, DL, 1993. Artificial Neural Networks for Land Cover Classification and Mapping. International Journal of Geographical Information Systems 7(2): 173-186.

Coleman, AM and LW Vail. 2007. An Adaptive Multi-Scale Watershed Characterization Approach Utilizing Geoinformatics and Self-Organizing 
Maps. In Proceedings of the American Geophysical Union, Fall Meeting. American Geophysical Union, San Francisco, California, December 10-15, 2007.

Conrad, $O$ and A Ringeler. 2007. System for Automated Geoscientific Analysis (SAGA), Release Candidate 2 Göttingen University, Göttingen, Germany. Accessed online August 12, 2006 at http://www.saga-gis.unigoettingen.de/html/index.php (undated webpage).

Costa-Cabral, M and SJ Burges. 1994. Digital Elevation Model Networks (DEMON): A Model of Flow Over Hillslopes for Computation of Contributing and Dispersal Areas. Water Resources Research 30(6):1681-1692.

Cowles, HC. 1911. The Causes of Vegetational Cycles. Annals of the Association of American Geographers 1(1):3-20.

Dai, E, SH Wu, SZ Shi, CK Cheung, and A Shaker. 2005. Modeling change-patternvalue dynamics on land use: An integrated GIS and artificial neural networks approach. Environmental Management 36(4):576-591.

Daly, C, RP Neilson, and DL Phillips. 1994. A Statistical-Topographic Model for Mapping Climatological Precipitation over Mountanious Terrain. Journal of Appplied Meteorology 33(2):140-158.

DeGruijter, JJ and AB McBratney. 1988. A modified fuzzy k means for predictive classification. In: HH Bock (ed.), Classification and Related Methods of Data Analysis Classification and Related Methods of Data Analysis. Elsevier Science, Amsterdam.

Demuth, H and M Beale. 2007. Neural Network Toolbox. Mathworks, Inc., Natick, Massachusetts.

Dixon, B. 2005. Applicability of neuro-fuzzy techniques in predicting ground-water vulnerability: a GIS-based sensitivity analysis. Journal of Hydrology 309(1-4): 17-38.

Dubayah, R and PM Rich. 1995. Topographic Solar Radiation Models for GIS. International Journal of Geographical Information Systems 9(4):405-419.

Eastman, JR. 2006. IDRISI Andes (Version 15). Clark Labs, Clark University, Worcester, Maryland.

Ehsani, AH. 2007. Artificial Neural Networks: Application in Morphometric and Landscape Features Analysis, KTH, Royal Institute of Technology, Stockholm, pp. 53.

Ermini, L, F Catani, and N Casagli. 2005. Artificial Neural Networks applied to landslide susceptibility assessment. Geomorphology 66(1-4):327-343. 
ESRI, 2007. ArcGIS Desktop and Arc/INFO Workstation (Version 9.2). Environmental Systems Research Institute, Inc., Redlands, California.

Evans, F. 1998. An Investigation into the Use of Maximum Likelihood Classifiers, Decision Trees, Neural Networks and Conditional Probabilistic Networks for Mapping and Predicting Salinity. MSc thesis. Curtin University of Technology, Perth, pp. 99.

Figueiredo, M and C Gloster. 1998. Implementation of a Probabilistic Neural Network for Multi-spectral Image Classification on an FPGA based Custom Computing Machine. In Proceedings of the Vth Brazilian Symposium on Neural Networks. IEEE Computer Society, Washington, District of Columbia.

Filippi, JB and P Bisgambiglia. 2004. JDEVS: an implementation of a DEVS based formal framework for environmental modelling. Environmental Modelling \& Software 19(3):261-274.

Fisher, RA. 1936. The Use of Multiple Measurements in Taxonomic Problems. Annals of Eugenics 7(Part II):179-188.

Forman, RTT and M Godron. 1986. Landscape Ecology. Wiley, New York, pp. 640.

Freeman, JA and DM Skapura. 1991. Neural Networks: Algorithms, Applications, and Programming Techniques. Computation and Neural Systems Series. Addison-Wesley Longman Publishing, Redwood City, California, pp. 401.

Fritzke, B and HS Loos. 1997. Some Competitive Learning Methods, Institute for Neural Computation, Ruhr-Universitat, Bochum, Germany.

Gleason, HA. 1927. Further Views on the Succession-Concept. Ecology 3(2):299326.

Gomes, H, AB Ribeiro, and V Lobo. 2007. Location model for CCA-treated wood waste remediation units using GIS and clustering methods. Environmental Modelling and Software 22(2007):1788-1795.

Govindaraju, RS and AR Rao. 2000. Artificial Neural Networks in Hydrology. Water Science and Technology Library, 36. Kluwer Academic Publishers, Dordrecht, Germany, pp. 329.

Govindaraju, RS and B Zhang. 2000. Radial-Basis Function Networks. In: Artificial Neural Networks in Hydrology. R.S. Govindaraju and A.R. Rao (eds.), Kluwer Academic Publishers, Dordrecht, Germany, pp. 329.

Hargrove, WW and RJ Luxmoore. 1998. A New High-Resolution National Map of Vegetation Ecoregions Produced Empirically Using Multivariate Spatial Clustering. In Proceedings Environmental Systems Research Institute User Conference. Environmental Systems Research Institute, Palm Springs, California. Accessed January 6, 2007 at 
http://gis.esri.com/library/userconf/proc98/proceed/TO350/PAP333/P333.HT $\underline{\text { M }}$ (last updated April 1, 1998).

Hilbert, DW and B Ostendorf. 2001. The utility of artificial neural networks for modelling the distribution of vegetation in past, present and future climates. Ecological Modelling 146(1-3):311-327.

Homer, C, C Huang, L Yang, B Wylie and M Coan. 2004. Development of a 2001 National Landcover Database for the United States. Photogrammetric Engineering and Remote Sensing 70(7):829-840.

Hopfield, JJ. 1982. Neural Networks and Physical Systems with Emergent Collective Computational Abilities. Proceedings of the National Academy of Scientists(79):2554-2558.

Horn, DR. 1988. Annual flow statistics for ungaged streams in Idaho. American Society of Civil Engineers, Journal of Irrigation and Drainage Engineering, 114(3):463-474.

Hortness, JE and C Berenbrock. 2001. Estimating Monthly and Annual Streamflow Statistics at Ungaged Sites in Idaho. Water-Resources Investigations Report 01-4093, United States Geological Survey, Boise, Idaho.

Hsieh, BB and MR Jourdan. 2006. Watershed Similarity Analysis for Military Applications Using Supervised-Unsupervised Artificial Neural Networks. In Proceedings of the 25th Army Science Conference, Orlando, Florida.

Joy, MK and RG Death. 2004. Predictive modelling and spatial mapping of freshwater fish and decapod assemblages using GIS and neural networks. Freshwater Biology 49(8):1036-1052.

Kecman, V. 2001. Learning and Soft Computing. MIT Press, Cambridge, Massachusetts, pp. 541.

Kjelstrom, LC. 1998. Methods for Estimating Selected Flow-duration and FloodFrequency Characteristics at Ungaged Sites in Central Idaho. U.S. Geological Survey Water-Resources Investigations Report 94-4120, United States Geological Survey, Boise, Idaho.

Kjelstrom, LC and RL Moffatt. 1981. A Method of Estimating Flood-frequency Parameters for Streams in Idaho: U.S. Geological Survey Open-File Report 81-909, United States Geological Survey, Boise, Idaho.

Köthe, R and F Lehmeier. 1996. SARA - System zur Automatischen Relief-Analyse, Benutzerhandbuch, Geogr. Inst., University of Göttingen, Göttingen, Germany.

Kohonen, T. 1982. Self-organized formation of topologically correct feature maps. Biological Cybernetics, 43(1):59-69. 
Kohonen, T. 2001. Self-Organizing Maps. Springer-Verlag, Berlin, Germany, pp. 501.

Kohonen, T, J Hynninen, J Kangas and J Laaksonen, J. 1996. SOM_PAK: The SelfOrganizing Map Program Package. Technical Report A31, FIN-02150, Helsinki University of Technology, Espoo, Finland.

Lenz, R and D Peters. 2006. From data to decisions - Steps to an applicationoriented landscape research. Ecological Indicators 6(1):250-263.

Lin, GF and LH Chen. 2005. Time series forecasting by combining the radial basis function network and the self-organizing map. Hydrological Processes 19(10):1925-1937.

Lin, GF and LH Chen. 2006. Identification of Homogeneous Regions for Regional Frequency Analysis using the Self-Organizing Map. Journal of Hydrology 324(1-4):1-9.

Lingireddy, S and GM Brion (eds.). 2005. Artificial Neural Networks in Water Supply Engineering. American Society of Civil Engineers, Reston, Virginia, pp. 173.

Lioubimtseva, E and P Defourny. 1999. GIS-based landscape classification and mapping of European Russia. Landscape and Urban Planning 44(2-3):63-75.

Lipscomb, SW. 1998. Hydrologic Classification and Estimation of Basin and Hydrologic Characteristics of Subbasin in Central Idaho, U.S. Geological Survey Professional Paper 1604. United States Geological Survey, Boise, Idaho, pp. 49.

Lloyd, SP. 1982. Least Squares Quantization in PCM. IEEE Transactions on Information Theory 28(2):129-137.

Loos, HS and B Fritzke. 1998. DemoGNG (Version 1.5). Institute for Neural Computation, Ruhr-Universität Bochum, Bochum, Germany. http://www.neuroinformatik.ruhr-uni bochum.de/ini/VDM/research/gsn/DemoGNG/tex/DemoGNG/DemoGNG.html Accessed August 18, 2007 (last updated October 19, 1998).

Loveland, TR and AS Belward. 1997. The IGBP-DIS Global $1 \mathrm{~km}$ Land Cover Data Set, DISCover First Results. International Journal of Remote Sensing 18(5):3,289-3,295.

MacQueen, JB. 1967. Some Methods for Classification and Analysis of Multivariate Observations. In Proceedings of the 5th Berkeley Symposium on Mathematical Statistics and Probability. University of California Press, Berkeley, pp. 281-297.

Mangiameli, P, SK Chen and D West. 1996. A comparison of SOM neural network and hierarchical clustering methods. European Journal of Operational Research, 93(2):402-417. 
Marrone, P. 2007. Java Object Oriented Neural Engine (JOONE). Accessed March 13, 2007 at http://www.jooneworld.com (last updated January 21, 2008).

Matthes-Sears, U, WC Matthes-Sears, SJ Hastings and WC Oechel. 1988. The Effects of Topography and Nutrient Status on the Biomass, Vegetative Characteristics, and Gas Exchange of Two Deciduous Shrubs on an Arctic Tundra Slope. Arctic and Alpine Research, 20(3):342-351.

McBratney, AB and JJ DeGruijter. 1992. Spatial predictions and mapping of continuous soil classes. Geoderma 54(1992):39-64.

McCulloch, WS and W Pitts. 1943. A Logic Calculus of the Ideas Immanent in Nervous Activity. Bulletin of Mathematical Biophysics 5:115-113.

Michie, D, DJ Spiegelhalter and CC Taylor (eds.). 1994. Machine Learning, Neural and Statistical Classification. Ellis Horwood, London, England, pp. 289.

Miller, DA and RA White. 1998. A Conterminous United States Multi-Layer Soil Characteristics Data Set for Regional Climate and Hydrology Modeling. Earth Interactions 2(2):1-26.

Minasny, B and AB McBratney. 2002. FuzME (Version 3.5). The University of Sydney, Australian Centre for Precision Agriculture, Sydney, Australia. Accessed December 5, 2006 at http://www.usyd.edu.au/su/agric/acpa/fkme/program.html (last updated December, 2003).

Moore, ID, RB Grayson and AR Ladson. 1991. Digital Terrain Modeling: A Review of Hydrological, Geomorphological, and Biological Applications. Hydrological Processes 5(1):3-30.

Moradkhani, H, KI Hsu, HV Gupta, and S Sorooshian. 2004. Improved streamflow forecasting using self-organizing radial basis function artificial neural networks. Journal of Hydrology 295(1-4):246-262.

NASA, 2007. MODIS Atmosphere, Filled Land Surface Albedo Product, MOD43B3. National Aeronautics and Space Administration, Goddard Space Flight Center, Greenbelt, Maryland.

Nellemann, C and G Fry. 1995. Quantitative Analysis of Terrain Ruggedness in Reindeer Winter Grounds. Arctic 48(2):172-176.

Nikolaev, N. 2008. Neural Networks, Probabilistic Neural Networks. University of London, London. Accessed April 12, 2008 at http://homepages.gold.ac.uk/nikolaev/311pnn.htm (undated webpage).

Njoku, E. 2007. AMSR-E/Aqua L2B Surface Soil Moisture, Ancillary Parms, \& QC EASE-Grids V002. National Snow and Ice Data Center, Boulder, Colorado. Accessed February 16, 2007 at 
http://nsidc.org/data/docs/daac/ae land 12b soil moisture.gd.html (last updated October, 2007).

NOHRSC, 2007. Snow Data Assimilation System (SNODAS), National Snow and Ice Data Center, Boulder, Colorado. National Operational Hydrologic Remote Sensing Center, Chanhassen, Minnesota. Accessed December 5, 2007 at http://nsidc.org/data/g02158.html (undated webpage).

NRCS, 2007. SNOTEL Site Information and Reports for Hoodoo Basin, Natural Resources Conservation Service, National Weather and Climate Center, Portland, Oregon. Accessed September 3, 2007 at http://www.wcc.nrcs.usda.gov/snotel/snotel.pl?sitenum=530\&state $=\mathrm{mt}$ (undated webpage).

NWS, 2007. National Digital Forecast Database. United States National Oceanic and Atmopheric Administration, National Weather Service, Office of Climate, Weather, and Water Services, Silver Spring, Maryland. Accessed February 1, 2007 at http://www.weather.gov/ndfd/index.htm (last updated May 9, 2007).

OGC, 2007. Sensor Web Enablement, Open Geospatial Consortium Standards. Accessed December 5, 2007 at http://www.opengeospatial.org/ogc/marketstechnologies/swe (last updated May 1, 2008).

Olaya, V. 2004. A Gentle Introduction to SAGA GIS. University of Göttingen, Germany, Göttingen. Accessed July 15, 2006 at http://www.saga-gis.unigoettingen.de/html/modules.php?op=modload\&name=Sections\&file=index\&re $\mathrm{q}=$ viewarticle\&artid=4\&page $=1$ (undated webpage).

Osinski, E. 2003. Operationalisation of a landscape-oriented indicator. Agriculture Ecosystems \& Environment 98(1-3):371-386.

Park, YS, IS Kwak, TS Chon, JK Kim and SE Jorgensen. 2001. Implementation of artificial neural networks in patterning and prediction of exergy in response to temporal dynamics of benthic macroinvertebrate communities in streams. Ecological Modelling. 146(1-3):143-157.

Pelleg, D. 2004. K-means and KD-trees Resources. Carnegie Mellon University, School of Computer Science, Pittsburgh, Pennsylvania. Accessed November 20, 2007 at http://www.cs.cmu.edu/ dpelleg/kmeans.html (last updated August 13, 2004).

Perus, I and A Krajinc. 1996. AiNet: A Neural Network Application for 32-bit Windows Environment (Version 1.25), User's Manual. Celje, Solvenia. Accessed February 6, 2007 at http://www.winsite.com/bin/Info?500000014622 (undated webpage).

Peucker, TK and DH Douglas. 1975. Detection of Surface-Specific Points by Local Parallel Processing of Discrete Terrain Elevation Data. Computer Graphics and Image Processing 4(4):375-387. 
Planchon, O and F Darboux. 2001. A Fast, Simple, and Versatile Algorithm to Fill the Depressions of Digital Elevation Models. Catena 46(2):159-176.

Principe, JC, NR Euliano, and WC Lefebvre. 2000. Neural and Adaptive Systems: Fundamentals Through Simulations. Wiley, New York.

Principe, JC and WC Lefebvre. 2007. NeuroSolutions (Version 5). NeuroDimension, Inc., Gainesville, Florida.

Provost, F and R Kohavi. 1998. On Applied Research in Machine Learning. Machine Learning 30(2-3):127-132.

Quillian, EW and WA Harenberg. 1982. An Evaluation of Idaho Stream-gaging Networks. U.S. Geological Survey Open File Report 82-865, United States Geological Survey, Boise, Idaho.

Rao, AR and VV Srinivas (eds.). 2008. Regionalization of Watersheds: An Approach Based on Cluster Analysis. Water Science and Technology Library, Volume 58, Springer Science+Business Media B.V., New York.

Richards, JA. 1986. Remote Sensing Digital Image Analysis: An Introduction. Springer-Verlag, New York, pp. 281.

Richards, JA and X Jia. 1999. Remote Sensing and Digital Image Analysis. Springer, New York, pp. 340.

Richards, JA and J Xiuping. 2006. Remote Sensing Digital Image Analysis, $4^{\text {th }}$ Edition, Springer-Verlag, New York, pp. 439.

Riley, SJ, SD DeGloria and R Elliot, R. 1999. A Terrain Ruggedness Index that Quantifies Topographic Heterogeneity. Intermountain Journal of Sciences, 5(1-4):23-27.

Rohwer, R, M Wynne-Jones and F Wysotzki. (eds.), 1994. Neural Networks. Machine Learning, Neural, and Statistical Classification. Ellis Horwood, London, England, pp. 289.

Rumelhart, DE, GE Hinton and RJ Williams. 1986. Learning Internal Representations by Error Propagation. In: Parallel Distributed Processing: Explorations in the Microstructure of Cognition. DE Rumelhart and JL McCelland (eds.), Massachusetts Institute of Technology, Cambridge, Massachusetts, pp. 318362.

Russell, E and H Ochis. 1997. Mitigation Methods for Systematic Errors in USGS DEMs. Computer Terrain Mapping, Inc., Boulder, Colorado. Accessed November 19, 2007 at http://www.ctmap.com/assets/pdfprojects/filtering wp.pdf (undated webpage). 
Sawatzky, DL, GL Raines, GF Bonham-Carter and CG Looney. 2004. ARCSDM3.1: ArcMAP Extension for Spatial Data Modelling Using Weights of Evidence, Logistic Regression, Fuzzy Logic and Neural Network Analysis. Natural Resources Canada, Geological Survey of Canada, Ottawa, Canada. Accessed August 16, 2006 at http://www.ige.unicamp.br/sdm/ArcSDM31/ (last updated September 27, 2006).

Schmidt, J and A Hewitt. 2004. Fuzzy land element classification from DTMs based on geometry and terrain position. Geoderma 121(3-4):243-256.

Schmuker, M, F Schwarte, A Brück, E Proschak, Y Tanrikulu, A Givehchi, K Scheiffele, G Schneider. 2007. SOMMER: Self-Organizing Maps for Education and Research. Journal of Molecular Modeling. 13(1):225-228.

SFCC, 2007. The Gestalt Principles. Department of Graphic Design, Spokane Falls Community College, Spokane, Washington.

Shanmugam, P, YH Ahn, and S Sanjeevi. 2006. A comparison of the classification of wetland characteristics by linear spectral mixture modelling and traditional hard classifiers on multispectral remotely sensed imagery in southern India. Ecological Modelling 194(4):379-394.

Shaver, GR, KJ Nadelhoffer and AE Giblin. 1990. Biogeochemical Diversity and Element Transport in a Heterogeneous Landscape, the North Slope Alaska. In: Qualitative Methods in Landscape Ecology, MG Turner and RH Gardner (eds.), Springer-Verlag, New York, pp. 105-125.

Short, NM. 2006. Image Processing and Interpretation - Morro Bay, California. Remote Sensing Tutorial, National Aeronautics and Space Administration, Goddard Space Flight Center, Greenbelt, Maryland. Accessed November 19, 2007 at http://rst.gsfc.nasa.gov/Sect1/Sect1 19.html (undated webpage).

Smith, L. 1996. An Introduction to Neural Networks. University of Stirling, Department of Computing and Mathematics, Centre for Cognitive and Computational Neuroscience, Stirling, Scotland. Accessed October 13, 2007 at http://www.cs.stir.ac.uk/ Iss/NNIntro/InvSlides.html (webpage last updated October 25, 1996).

Snelder, TH and BJF Biggs. 2002. Multiscale River Environment Classification for Water Resources Management. Journal of the American Water Resources Association 38(5):1225-1239.

Sraj, M, M Brilly, L Globevnik, M Padeznik and M Mikos. 2006. The Dragonja River Experimental Watershed. In Proceedings of the European Geosciences Union, General Assembly 2006, Geophysical Research Abstracts, Vienna, Austria, April 2-7, 2006. Accessed March 11, 2008 at http://www.cosis.net/abstracts/EGU06/02653/EGU06-J-02653.pdf (undated webpage). 
StatSoft, 2003. Neural Networks. Stat Soft, Inc., Tulsa, Oklahoma. Accessed on October 12, 2007 at http://www.statsoft.com/textbook/glosa.html (undated webpage).

Stow, D, A Petersen, J Rogan, and J Franklin. 2007. Mapping burn severity of Mediterranean-type vegetation using satellite multispectral data. GIS Science \& Remote Sensing, 44(1):1-23.

Tarboton, DG. 1997. A New Method for the Determination of Flow Directions and Upslope Areas in Grid Digital Elevation Models. Water Resources Research 33(2):309-319.

Thomas, BE, HW Hjalmarson and SD Waltemeyer. 1994. Methods for Estimating Magnitude and Frequency of Floods in the Southwestern United States, United States Geological Survey Open-File Report 93-419. United States Geological Survey, Reston, Virginia.

Thomas, CA, WA Harenberg and JM Anderson. 1973. Magnitude and Frequency of Floods in Small Drainage Basins in Idaho, United States Geological Survey Water-Resources Investigations 7-73, United States Geological Survey, Boise, Idaho.

Thorson, TD, SA Bryce, DA Lammers, AJ Woods, JM Omernik, J Kagan, DE Pater and JA Comstock. 2003. Ecoregions of Oregon. United States Geological Survey, Reston, Virginia. Accessed on September 9, 2006 at http://www.epa.gov/wed/pages/ecoregions/or eco.htm (last updated October 2, 2007).

Tso, B and PM Mather. 2001. Classification Methods for Remotely Sensed Data. Taylor and Francis, New York, pp. 352.

Turner, MG. 1989. Landscape ecology: the effect of pattern on process. Annual Review of Ecological Systems 20(1989):171-87.

Turner, MG, RH Gardner and RV O'Neill. 2003. Landscape Ecology in Theory and Practice: Pattern and Process. Springer-Verlag, New York, pp. 404.

UCI, 2007. Machine Learning Repository, Statlog - Landsat Satellite Data Set. University of California Irvine. Accessed on December 8, 2007 at http://archive.ics.uci.edu/ml/datasets/Statlog+\%28Landsat+Satellite $\% 29$ (undated webpage).

Vesanto, J, J Himberg, E Alhoniemi, and J Parhankangas. 2000. SOM Toolbox for Matlab 5. Helsinki University of Technology, Laboratory of Computer and Information Science, Espoo, Finland.

Vrieling, A, SC Rodrigues, H Bartholomeus and G Sterk. 2007. Automatic identification of erosion gullies with ASTER imagery in the Brazilian Cerrados. International Journal of Remote Sensing 28(12):2723-2738. 
Wang, HB and K Sassa. 2006. Rainfall-induced landslide hazard assessment using artificial neural networks. Earth Surface Processes and Landforms, 31(2):235247.

Ward, JH. 1963. Hierarchical grouping to optimize an objective function. Journal of American Statistical Association 58(301):263-244.

Wardrop, DH, JA Bishop, M Easterlin, K Hychka, W Myers, GP Patil, C Taillie. 2005. Use of landscape and land use parameters for classification and characterization of watersheds in the mid-Atlantic across five physiographic provinces. Environmental and Ecological Statistics 12(2):209-223.

Watts, D. 1971. Principles of Biogeography. McGraw-Hill, New York, pp. 401.

Wetherill, G. 1986. Regression Analysis with Applications. Chapman and Hall, New York, pp. 311.

Wiesner, A. 2008. Applied Multivariate Statistical Analysis. Department of Statistics, Penn State University, State College, Pennsylvania. Accessed April 7, 2008 at http://www.stat.psu.edu/ jglenn/stat505/index.html (undated webpage).

Willshaw, DJ and C von der Malsburg. 1976. How patterned neural connections can be setup by self-organization. Proceedings of the Royal Society London, Vol. B194, pp. 431-445.

Wilson, JP and JC Gallant (eds.). 2000. Terrain Analysis: Principles and Applications. Wiley, Hoboken, New Jersey, pp. 479.

Worboys, MF and M Duckham. 2004. GIS: A Computing Perspective. CRC Press, Boca Raton, Florida, pp. 426.

WRCC, 2007. Idaho Annual Precipitation Summary, Western Regional Climate Center, Desert Research Institute, Reno, Nevada. Accessed September 3, 2007 at http://www.wrcc.dri.edu/htmlfiles/id/id.ppt.ext.html (undated webpage).

Yuras, G. 1996. Remote Sensing. Center for Oceanographic Research in the Eastern South Pacific, University of Concepción. Accessed November 19, 2007 at http://copas.udec.cl/ gabriel/tutoriales/rsnote/contents.htm (undated webpage).

Zevenbergen, LW and CR Thorne. 1987. Quantitative Analysis of Land Surface Topography. Earth Surface Processes and Landforms, 12(1):47-56.

Ziadat, FM. 2005. Analyzing Digital Terrain Attributes to Predict Soil Attributes for a Relatively Large Area. Soil Science Society of America Journal 69(2005):1590-1599. 


\subsection{Appendix A}

Several programs were written which comprise the overall function of the ALCP. Some of these programs are specific to generating data for use in the ALCP. The following provides a description of each program and the sequence in which they are run.

watershed setup.aml

Produces the following datasets using input DEM data: Filled-Sink DEM, FlowDirection, Flow-Accumulation, Slope, Aspect, Hillshade, Hypsometric GRID, Upslope Surface Contributing Area, Compound Topographic Index, Modified Compound Topographic Index, Solar Radiation Index, and Topographic Roughness Index

watershed saga.csh

UNIX C-shell script to launch the SAGA GIS processor which calculates primary and secondary terrain attributes including subbasins, channel networks, curvatures, wetness index, and more.

strm slp.aml

Calculates average stream slope for a basin based on generated channel networks and catchment boundaries.

solarcalc.csh

UNIX C-shell script to launch the SAGA GIS processor for calculating daily solar radiation and insolation values.

dump sb values.aml ALCP process which extracts descriptive statistical values and full data vectors for each subbasin including static data (i.e. topo, slope, aspect, etc.) and dynamic or temporal data. This data is extracted and prepares input vector files for unsupervised classification with Self-Organizing Maps (SOM).

\section{runsom.sh}

UNIX shell script batch file processor to execute SOMMER code for unsupervised classication.

sompostproc.sh

UNIX Shell script to post-process SOMMER output data for purpose of rejoining to GIS database.

joinsom.aml Joins SOM class data back into the GIS by using the spatial container unique-id as the index (join) value. This script relies upon an external AML called 'ascii2info.aml' which is also included herein. 


\section{[ - watershed_setup.aml - ]}

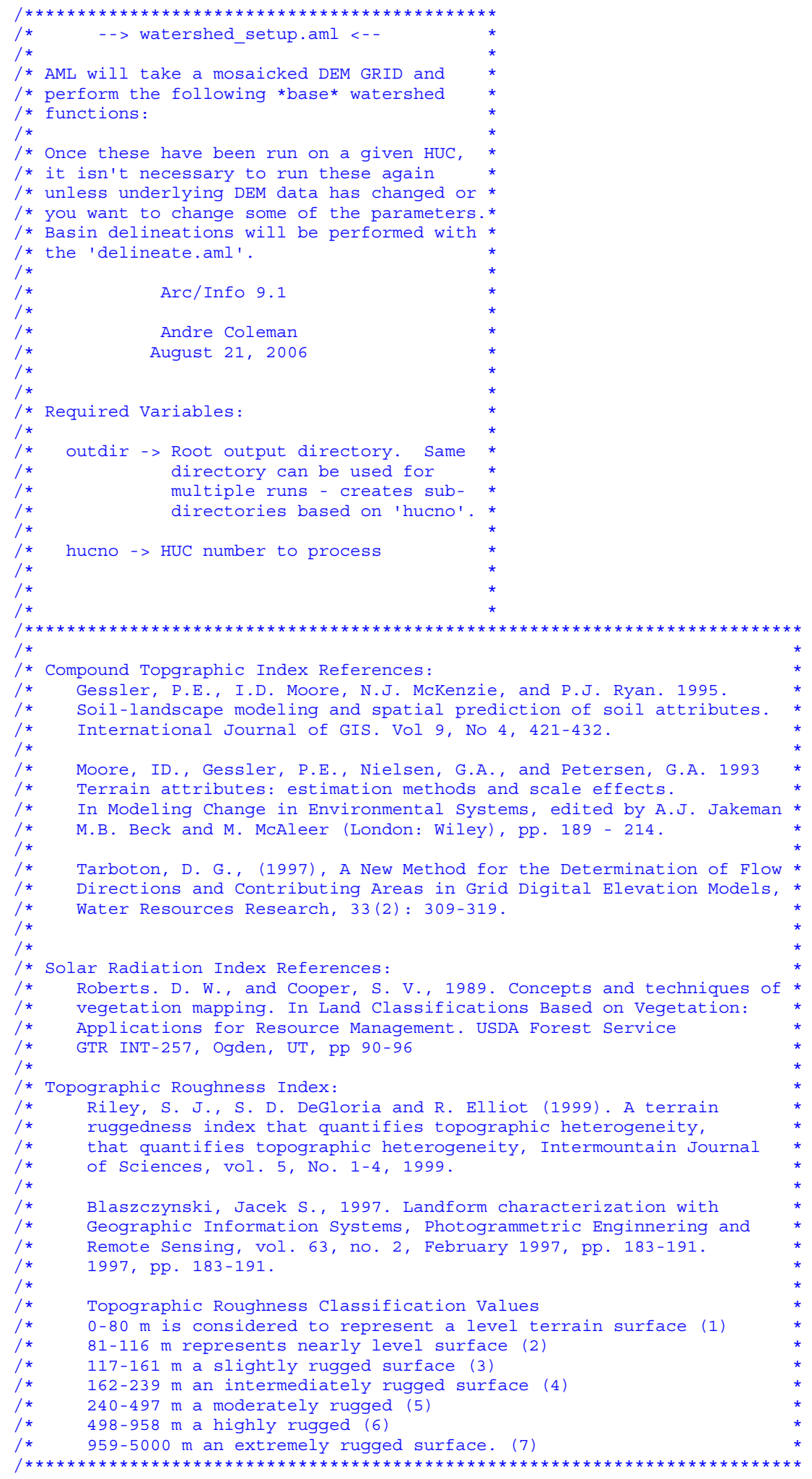

\&args outdir hucno clean

\&if [null \%hucno\%] \&then \&do

\&type /\& Usage: WATERSHED_SETUP <ROOT OUTPUT DIRECTORY> <HUC NUMBER> \{CLEAN\} \&type

\&type NOTE: 


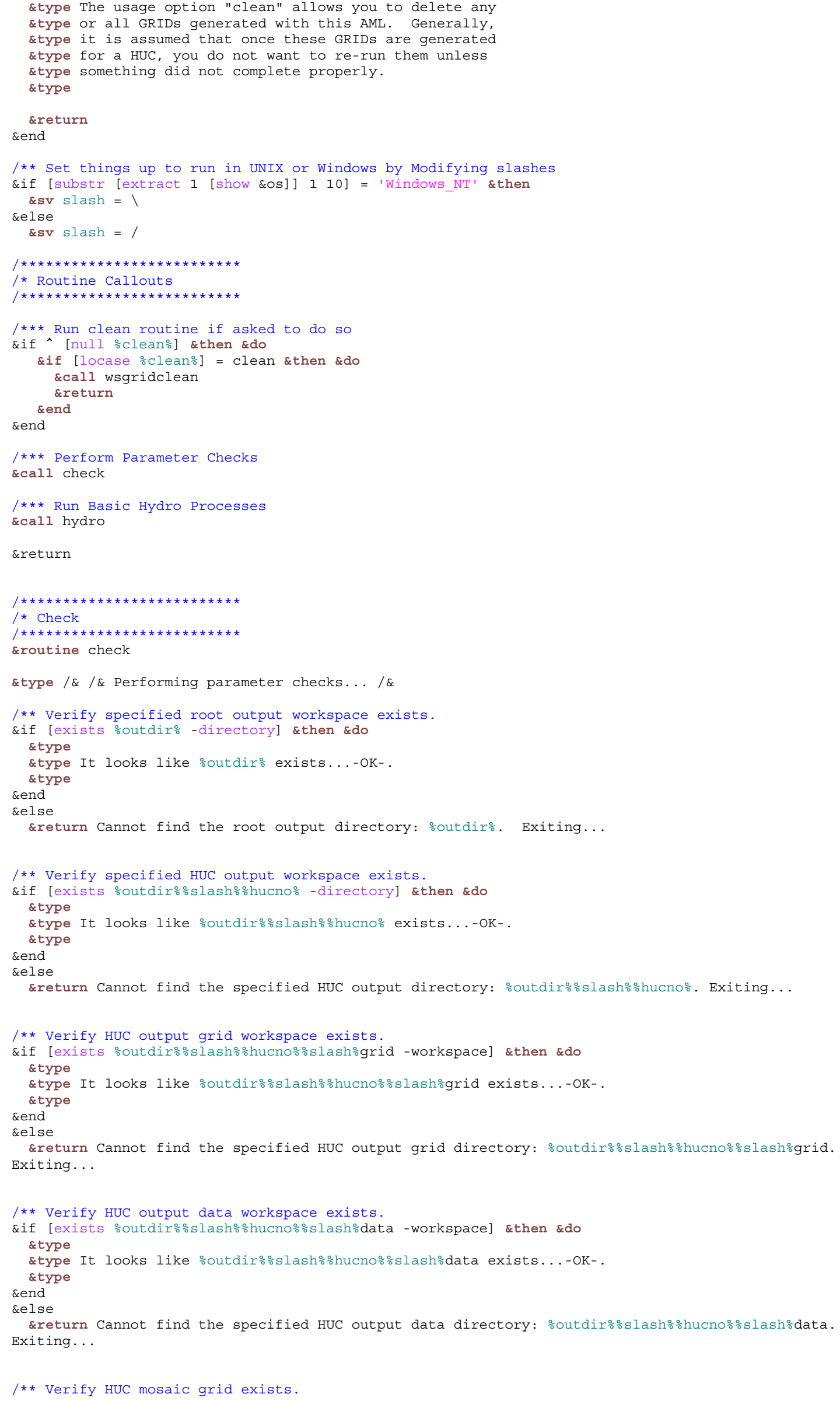




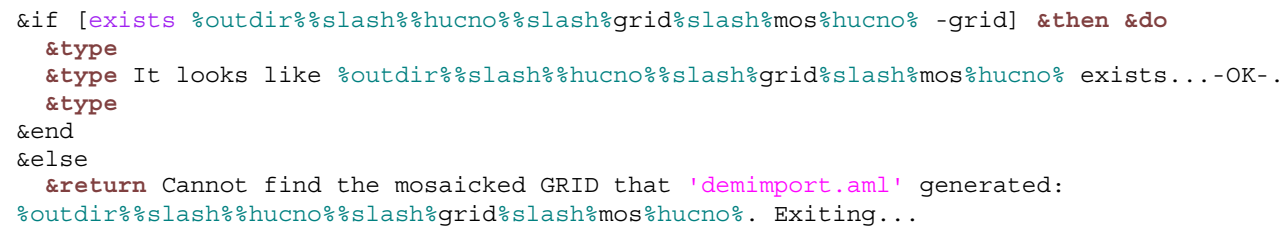

\&type /\& Finished running parameter checks...proceeding./\&

\&return

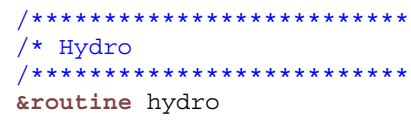

\&type /\& Running Basic Hydro setup for HUC \%hucno\%.../\&

/*** Fill Elevation Pits

$/ *$ Run only if this does not exist already

\&if $\wedge$ [exists $\%$ outdir\%:slash\%\%hucno\%slash\%grid\%slashomos\%hucno\%fl -grid] \&then \&do \&type /\& Filling elevation pits in mos\%hucno\%.../\&

grid

display 0

fill \%outdir\%:slash\% $\%$ heno\% $\%$ slash\%grid\%slash\%mos\%hucno\%

outdir\%:slash\%弓hucno\%slash\%grid\%slash\%mos\%hucno\%fl SINK

quit

send

selse \&do

\&type /\&Filled elevation GRID already exists...skipping.

stype Run this AML with the -clean- option if you want to regenerate this. \&end

/*** Create Flow Direction GRID

$/ *$ Run only if this does not exist already

\&if ${ }^{\wedge}$ [exists \%outdir\%:slash\%\%hucno\%:slash\%grid\%slashomos\%hucno\%fd -grid] \&then \&do \&type /\&Creating Flow Direction GRID using mos\%hucno\% Filled DEM.../\&

grid

display 0

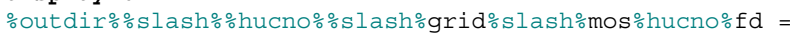

flowdirection (\%outdir\%slash\%弓hucno\%slash\%grid\%slash\%mos\%hucno\%fl, \#, NORMAL) quit

send

\&else \&do

\&type /\&Flow Direction GRID already exists...skipping.

\&type Run this AML with the -clean- option if you want to regenerate this. \&end

/*** Create Flow Accumulation GRID

$/ *$ Run only if this does not exist already

\&if ‘ [exists \%outdir\%:slasho\%hucno\%slash\%grid\%slashomos\%hucno\%fa -grid] \&then \&do \&type /\&Creating Flow Accumulation GRID using mos $\%$ hucno\%s Flow Direction GRID.../\& grid

display 0

\%outdir\%:

flowaccumulation (\%outdir\%:slash\%弓hucno\%slash\%grid\%slash\%mos\%hucno\%fd, \#)

quit

\&end

\&else \&do

\&type /\&Flow Accumulation GRID already exists...skipping.

\&type Run this AML with the -clean- option if you want to regenerate this. \&end

/*** Generate Slope GRID

/*Run only if this does not exist already

\&if $\wedge$ [exists $\%$ outdir\%\%slasho\%hucno\%:slashogrid\%slashomos\%hucno\%sl -grid] \&then \&do \&type /\&Creating Slope GRID using mos\%hucno\% as a source.../\&

grid

display 0

\%outdir\%:slash\%hucno\%:slash\%grid\%slash\%mos\%hucno\%sl =

slope (\%outdir\%slash\% \%hucno\%slash\%grid\%slash\%mos\%hucno\%, DEGREE) quit

\&end

\&else \&do

\&type /\&Slope GRID already exists...skipping.

\&type Run this AML with the -clean- option if you want to regenerate this. \&end

/*** Generate Aspect GRID

/*Run only if this does not exist already

\&if $\wedge$ [exists $\%$ outdir\%\%slash\%弓hucno\% $\%$ sash\%grid\%slashomos\%hucno\%as -grid] \&then \&do \&type /\&Creating Aspect GRID using mos\%hucno\% as a source.../\&

grid

display 0 


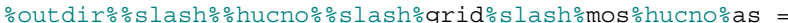
aspect (\%outdir\% $\%$ slash $\%$ hucno\% $\%$ lash\%grid $\%$ sash $\%$ mos $\%$ heno\%)

quit

\&end

\&else \&do

\&type /\&Aspect GRID already exists...skipping.

stype Run this AML with the -clean- option if you want to regenerate this. \&end

/*** Generate Hillshade GRID for visibility purposes

$/ *$ Run only if this does not exist already

\&if ^ [exists \%outdir\%\%slash\%弓ucno\%slash\%grid\%slash\%mos\%hucno\%hs -grid] \&then \&do

\&type /\&Creating hillshade GRID using mos\%hucno\% as a source.../\&

hillshade \%outdir\%:slash\% \% hucno\%

outdir\%:slash\%\%ucno\%slash\%grid\%slash\%mos\%hucno\%hs \# \# \# 2.5 /*135 \# ALL 2.5

\&end

\&else \&do

\&type /\&Hillshade GRID already exists...skipping.

\&type Run this AML with the -clean- option if you want to regenerate this. \&end

/*** Generate Compound Topographic Index GRID

$/ *$ Run only if this does not exist already

\&if $\wedge$ [exists \%outdir\%:slash\%\%hucno\%slashogrid\%slashomos\%hucno\%ti -grid] \&then \&do \&type /\&Creating Compound Topographic Index GRID using mos\%hucno\% as a source.../\&

/*Run Cleanup Check First

\&do cover \&list mos\%hucno\%sl mos\%hucno\%st mos\%hucno\%sC mos\%hucno\%ua

\&if [exists $\%$ outdir\%응 \&type \Removing \%outdir\%\%slash\%弓冫ucno\% \&end kill \%outdir\% \%

\&end /*End of Do List routine to remove temporary GRIDs

$/ * *$ Determine Cell-Size of DEM

\&describe \%outdir\%: \&sv cellsize = \%GRD $\$ D X \%$

grid

display 0

\&type /\& Converting Slope Degrees to Radians Using: slope * (pi / 2) / $90 / \&$

\&if [exists \%outdir\%\%slash\%\%hucno\%slash\%grid\%slash\%mos\%hucno\%sl -grid] \&then

kill \%outdir\%:slash\%弓ucno\%:slash\%grid\%slashomos\%hucno\%sl all

\%outdir\%:

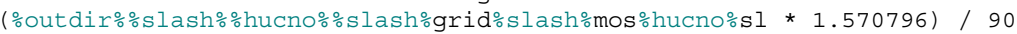

\&type /\& Calculating Tangent of Slope(radians) ... /\&

\&if [exists \%outdir\%:slash\%弓hucno\%slash\%grid\%slash\%mos\%hucno\%st -grid] \&then

kill \%outdir\%:slash\% \%ucno\%slash\%grid\%slash\%mos\%hucno\%st all

\%outdir\%:

$\tan (\%$ outdir\% $\%$ slash\% \% $\%$ cno\% $\%$ slash\%grid\%slash\%mos\%hucno\%s1)

\&type /\& Reclassing 0 Slope Values to .001.. /\&

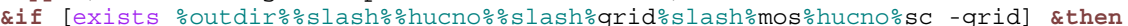

kill \%outdir\%:slash\%\%hcno\% \%lash\%grid\%slash\%mos\%hucno\%sc all

outdir\%: $\%$ lash\% \%

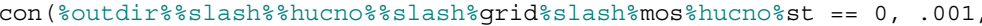

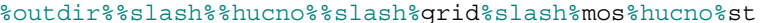

\&type /\& Calculating Upslope Surface Contributing Area... / \&

\&if [exists \%outdir\%slash\%\%hucno\%slash\%grid\%slash\%mos\%hucno\%ua -grid] \&then

kill \%outdir\%slash\% $\%$ hucno\%slash\%grid\%slash\%mos\%hucno\%ua all

\%outdir\%:

(\%outdir\% $\%$ slash응

\&type /\& Calculating Compound Topographic Index ln (a/tan (beta)) . . / \&

\%outdir\%:slash\% \%hucno\%slash\%grid\%slash\%mos\%hucno\%ti =

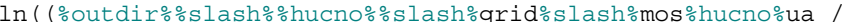

\%outdir\%:slash\%\%hucno\%slash\%grid\%slash\%mos\%hucno:sc))

\&type /\& Calculating Modified Compound Topographic Index (a/tan (beta)) .. / \&

\&if [exists \%outdir\%:slash\%弓hucno\%slash\%grid\%slash\%mos\%hucno\%mt -grid] \&then

kill \%outdir\% $\%$ slash\% $\%$ hucno\% $\%$ slash $\%$ grid\%slashomos\%hucno\%mt all

\%outdir\%:slash\%\%hucno\%slash\%grid\%slash\%mos\%hucno\%mt =

(\%outdir\%:slash\% \%ucno\%:slashogrid\%slash\%mos\%hucno\%ua /

outdir\%: $\%$ lash $\%$ hucno\% $\%$ slash\%grid\%slash\%mos\%hucno\%sc)

quit /*Out of GRID

/*Cleanup Temporary GRID

kill \%outdir\%:slash\%弓hucno\%slash\%grid\%slash\%mos\%hucno\%sl all

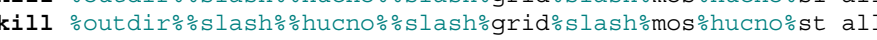

kill \%outdir\%:slash\%弓hucno\%slash\%grid\%slash\%mos\%hucno\%sc all

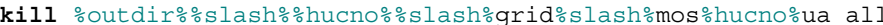
send

\&else \&do

\&type /\&Compound Topographic Index GRID already exists...skipping. 
\&type Run this AML with the -clean- option if you want to regenerate this. \&end

/*** Generate Solar Radiation Index GRID

/*Run only if this does not exist already

\&if ^ [exists \%outdir\%:slasho\%hucno\%:slash\%grid\%slash\%mos\%hucno\%sr -grid] \&then \&do

\&type /\&Creating Solar Radiation Index GRID using mos\%hucno\% as a source.../\&

grid

display 0

/*At this point we have to assume the Aspect GRID has been created.

/*Set non-aspects to null

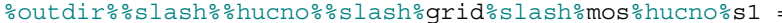

setnull (\%outdir\%: slash\% $\%$ hucno\% $\%$ slash\%grid\%slash\%mos $\%$ hucno\%as $<0$

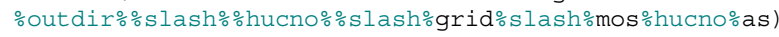

/*Calculate Solar Radiation Index

\%outdir\%: ( $\%$ outdir\%: 1 ash $\%$ hucno\% $\%$ slash\%grid\%slash\%mos\%hucno\%s1 - 30))

\%outdir\%: $\%$ lash $\%$ hucno $\%$ slash $\%$ grid $\%$ slash $\%$ mos $\%$ hucno\%sr =

\%outdir\%:slash\%\%hucno\%slash\%grid\%slash\%mos\%hucno\%s2 / 2

quit /*Out of GRID

/*Kill Intermediate GRIDs

kill \%outdir\%:slash\%\%hucno\%slash\%grid\%slash\%mos\%hucno\%sl all

\&end

kill \%outdir\%:slash\%\%hucno\%slash\%grid\%slash\%mos\%hucno\%s all

\&else \&do

\&type /\&Solar Radiation Index GRID already exists...skipping.

stype Run this AML with the -clean- option if you want to regenerate this. \&end

/*** Generate Topographic Roughness Index GRID

$/ *$ Run only if this does not exist already

\&if ${ }^{\wedge}$ [exists \%outdir\%:slasho\%hucno\%:slashogrid\%slashomos\%hucno\%tr-grid] \&then \&do

\&type /\&Creating Topographic Roughness Index GRID using mos\%hucno\% as a source.../\&

/*Change Directory

\&work $\frac{\circ}{\circ}$ outdir\% $\%$ slash $\%$ hucno\% $\%$ slash\%grid

grid

display 0

\&sv dem $=$ mos $\%$ hucno:

setwindow $\%$ dem $\%$ dem

setcell $\%$ dem\%

/*Calculate Standard Elevation Differences

DOCELI

mos $\%$ hucno\%t1 $=\left(\left(\operatorname{sqrt}\left(\frac{\circ}{\operatorname{dem} \%}(0,0)-\frac{\circ}{\operatorname{dedem}}(-1,-1)\right)\right) \sim\right.$

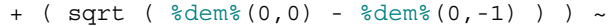

$+(\operatorname{sgrt}(\% \operatorname{dem} \div(0,0)-\% \operatorname{dem} \div(1,-1))) \sim$

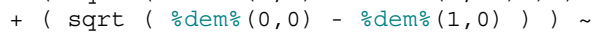

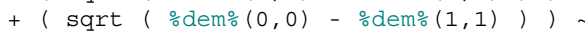

$+\left(\operatorname{sqrt}\left(\frac{\circ}{\partial} \operatorname{dem} \%(0,0)-\frac{\circ}{\operatorname{dedem} \%}(0,1)\right)\right) \sim$

$+(\operatorname{sgrt}(\% \operatorname{dem} \%(0,0)-\% \operatorname{dem} \%(-1,1))) \sim$

$+\left(\operatorname{sqrt}\left(\frac{\circ \operatorname{dem} \%}{0}(0,0)-\frac{\circ}{\circ} \operatorname{dem} \div(-1,0)\right)\right)$

END

/*Calculate the roughness index

mos $\%$ hucno $\%$ tr $=\operatorname{sqrt}($ mos $\%$ hucno $\% t 1)$

quit /*Out of GRID

/*Kill Intermediate GRID

kill mos\%hucno\%tl all

/*Return to Scripts Directory

\&work \%outdir\%

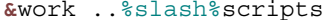

\&end

\&else \&do

\&type /\&Topographic Roughness Index GRID already exists...skipping.

\&type Run this AML with the -clean- option if you want to regenerate this. \&end

*** Generate Hypsometric GRID for visibility purposes

/*Run only if this does not exist already

\&if $\wedge$ [exists \%outdir\%\%slash\%\%hucno\% $\%$ slash\%grid\%slashomos\%hucno\%h -stack] \&then \&do

\&type /\&Creating Hypsometric GRID using mos\%hucno\% as a source.../\& 
\&sv outgrid $=\%$ outdir $\%$ slash $\%$ hucno $\%$ slash $\%$ grid

/*Pre-Cleanup

\&if [exists \%outgrid\%\%slash\%xxhill -grid] \&then

kill \%outgrid\%:slash\%xxhill all

\&if [exists \%outgrid\%oslash\%xxslice -grid] \&then

kill outgrido\%

\&if [exists \%outgrid\%\%slash\%xxillum -grid] \&then

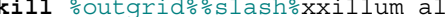

/***Routines Here Adapted from 'relief.aml' by D.W. Lamphear@ Redwood Sciences Laboratory, Arcata,

display 99991

mape image \%outgrido\%slash\%mos\%hucno\%

\&describe \%outgrid\%:slash\%mos\%hucno\%

\&sv tmpl grd $=$ [scratchname -directory]

\&if [exists \%outgrid\%\%slash\%०mp1_grd\% -grid] \&then

kill \%outgrid\%:slash\%०mpl_grd\% all

\&sv value grid = \%outgrid $\%$ slash $\%$ mos $\%$ hucno

\&sv elev grid = \%outgrido\%slash\%mos\%hucno\%

/*Normalize GRID

\&if $\% G R D \$ Z M I N \circ$ gt 0 \&then \&do

\%outgrid\%:slash\%tmpl grd\% = \%value grid\% - $\%$ GRD\$ZMIN

\&describe \%outgrid\%०

/*Calculate Factor

sv factor $=10000 / \%$ GRD\$ZMAX

\&type

stype The calculated factor is: \%factor\%

\&type

\&sv tmp2_grd = [scratchname -directory]

\%outgrid\% \%

\&end

selse

\&sv factor $=10000 /$ GRD\$ZMAX\%

\&sv tmp2 grd $=$ [scratchname -directory]

\&if [exists \%outgrid\%:slash\%

kill \%outgrid\%\%slash\%

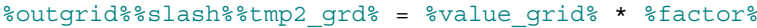

/**Generate LUT's

$/ *$ Color.lut

sif [exists \%outgrid\%:slash\%color.lut -file] \&then

\&sv delstat [delete \%outgrido\%slash\%color.lut -file]

/*Open file for writing

\&sv file $=$ outgrid\%\%slash\%color.lut

\&sv fileunit $=[$ open $\% f i l e \%$ openstatus -write $]$

\&if \%openstatus\% $<0$ \&then

\&return \&warning Error opening \%file\% for writing.

* Write to file

\&sv line = l1 8129242

\&sv linewrite $=$ [write $\%$ fileunit $\%$ line $\%$ ]

\&sv line $=1211315389$ '

\&sv linewrite $=$ [write $\%$ fileunit $\%$ Iine $\%$ ]

\&sv line $=131171701011$

\&sv linewrite $=$ [write $\%$ fileunit $\%$ Iine $\%$ ]

\&sv line = '4 149190113 '

\&sv linewrite $=$ [write $\%$ fileunit $\%$ Iine $\%$ ]

\&sv line = '5 $178214 \quad 117$ '

\&sv linewrite $=$ [write $\%$ fileunit $\%$ line $\%$ ]

\&sv line = '6 202226149 '

\&Sv linewrite $=$ [write $\%$ fileunit $\%$ Iine $\%$ ]

\&sv line $=17222238161$

\&sv linewrite $=$ [write $\%$ fileunit\% \%line $\%$ ]

\&sv line = $18242238 \quad 161$ '

\&sv linewrite $=$ [write $\%$ fileunit $\%$ Iine $]$

\&sv line = 19238222153

\&sv linewrite $=$ [write $\%$ fileunit $\%$ Iine\%]

\&sv line = '10 2422061331

\&sv linewrite $=$ [write $\%$ fileunit $\%$ ]

\&sv line = '11 234182129 '

\&sv linewrite $=$ [write $\%$ fileunit $\%$ line $\%$ 
\&sv line = '12 218157121 '

\&sv linewrite $=$ [write $\%$ fileunit: $\%$ line $\%$ ]

\&sv line $=$ '13 $194141 \quad 125$ '

\&sv linewrite $=$ [write $\%$ fileunit $\%$ Iine $\%$ ]

\&sv line = '14 214157145 '

\&sv linewrite $=$ [write $\%$ fileunit\% $\%$ line $\%$ ]

\&sv line $=$ '15 226174165 '

\&sv linewrite $=$ [write $\%$ fileunit\% $\%$ line $\%$ ]

\&sv line = '16 $222 \quad 186 \quad 182$ '

\&sv linewrite $=$ [write $\%$ fileunit $\%$ ine $\%$ ]

\&sv line = '17 238198 210'

\&sv linewrite $=$ [write $\%$ fileunit: $\%$ line $\%$ ]

\&sv line = '18 $255206226^{\prime}$

\&sv linewrite $=$ [write $\%$ fileunit $\%$ line $\%$ ]

\&sv line = '19 250218234 '

\&sv linewrite $=$ [write $\%$ fileunit $\%$ Iine $\%$ ]

\&sv line = $120255222 \quad 2301$

\&sv linewrite $=$ [write $\%$ fileunit $\%$ line\%]

\&sv line = '21 255230242 '

\&sv linewrite $=$ [write $\%$ fileunit\% \%line $\%$ ]

\&sv line = 122255242255 '

\&sv linewrite $=$ [write $\%$ fileunit\% $\%$ line $\%$ ]

\&sv line = '23 $255255255^{\prime}$

\&sv linewrite $=$ [write $\%$ fileunit $\%$ line $\%]$

/*Close file.

\&if [close \%fileunito] $>0$ \&then

\&return \&warning Unable to close \%file\%.

$/ *-*-*$

$/ *$ Value.lut

\&if [exists \%outgrid\%:slash\%value.lut -file] \&then

\&sv delstat [delete \%outgrido\%slashovalue.lut -file]

/*Open file for writing

\&sv file = \%outgrid\%\%slash\%value.lut

\&sv fileunit $=$ [open $\%$ file\% openstatus -write]

\&if \%openstatus\% $<>0$ \&then

\&return \&warning Error opening $\frac{\circ}{6}$ ile for writing.

/*Write to file

\&sv line $=1-100000000: 1{ }^{\prime}$

\&sv linewrite $=[$ write $\%$ fileunit $\%$ Iine $\%]$

\&sv line $=10000 \quad 0250: 2$

\&sv linewrite $=[$ write $\%$ fileunit $\%$ Iine $\%]$

\&sv line $=10250 \quad 0500: 31$

\&sv linewrite $=[$ write $\%$ fileunit $\%$ Iine $\%$

\&sv line $=10500 \quad 1000: 4$

\&sv linewrite $=$ [write $\%$ fileunit $\%$ line $\%$ ]

\&sv line $=110001500: 51$

\&sv linewrite $=[$ write $\%$ fileunit $\%$ line $\%]$

\&sv line $=115002000: 6^{\prime}$

\&sv linewrite $=[$ write $\%$ fileunit $\%$ line $\%$ ]

\&sv line $=120002500: 7$

\&sv linewrite $=[$ write $\%$ fileunit $\%$ Iine $\%]$

\&sv line $=125003000: 81$

\&sv linewrite $=$ [write $\%$ fileunit $\%$ ]ine

\&sv line $=130003500: 9$

\&sv linewrite $=$ [write $\%$ fileunit\% \%line\%]

\&sv line $=135004000: 101$

\&sv linewrite $=[$ write $\%$ fileunit $\%$ line

\&sv line $=140004500: 11$ '

\&sv linewrite $=$ [write $\%$ fileunit $\%$ line\%]

\&sv line $=145005000: 12$

\&sv linewrite $=[$ write $\%$ fileunit $\%$ Iine $]$

\&sv line $=150005500: 131$

\&sv linewrite $=[$ write $\%$ fileunit $\%$ Iine

\&sv line $=155006000: 14$

\&sv linewrite $=[$ write $\%$ fileunit $\%$ Iine $\%]$

\&sv line $=160006500: 15$

\&sv linewrite $=[$ write $\%$ fileunit $\%$ line $\%$ ]

\&sv line $=165007000: 16^{\prime}$

\&sv linewrite $=$ [write $\%$ fileunit $\%$ Iine $\%$

\&sv line $=170007500: 17$

\&sv linewrite $=$ [write $\%$ fileunit $\%$ ]ine

\&sv line $=175008000: 181$

\&sv linewrite $=[$ write $\%$ fileunit $\%$ Iine $\%$ ]

\&sv line $=180008500: 19$

\&sv linewrite $=$ [write $\%$ fileunit $\%$ line $\%$ ]

\&sv line $=185009000: 201$

\&sv linewrite $=$ [write $\%$ fileunit $\%$ ]ine $\%$ ]

\&sv line $=190009500: 21$ 


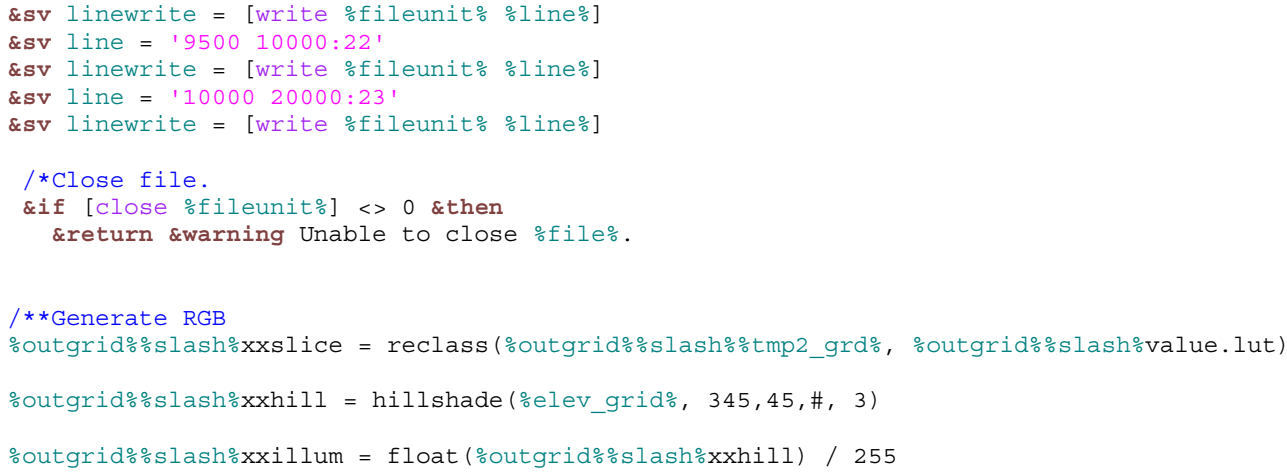

/*Create GRID Stack

makestack \%outgrid\%\% \%outgrid\%:slash\%mos\%hucno\%h2 \%outgrid\%:slash\%mos $\%$ hucno $\%$ h3

/*Display Resulting Hypso

mapextent \%elev_grid\%

gridcomposite rgb \%outgrid\%:slash\%mos\%hucno\%h1 \%outgrid\%:slash\%mos\%hucno\%h2 \%outgrid\%:slash\%mos\%hucno\%h3 identity

\&pause \&seconds 3

/*Cleanup

kill \%outgrid\%\%slash\%xxhill al

kill \%outgrid\%:slashoxxslice all

kill \%outgrid\%:slashoxxillum all

\&if [exists \%outgrid\%\%slasho\%tmpl_grd\% -grid] \&then

kill \%outgrid\%\%slash\%:tmp1_grd\% all

\&if [exists \%outgrid\%:slasho\%tmp2 grd\% -grid] \&then

kill \%outgrid\%: 1 lasho\%tmp 2 grd\% all

quit /*Out of GRID

/*Convert GRID to Image

sif [exists \%outgrido\%slashomos\%hucno\%h.tif -file] \&then \&do \&sv delstat [delete outgrid\%oslashomos\%hucno\%h.tif -file] end \&sv delstat [delete \%outgrid\%olash\%mos\%hucno\%h,tfw -file]

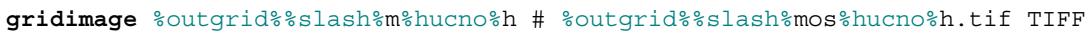

send

\&else \&do

\&type /\&Hypsometric GRID already exists...skipping.

stype Run this AML with the -clean- option if you want to regenerate this. send

/*** User Information Message

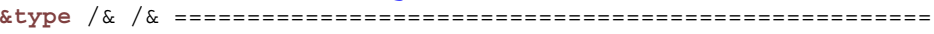

stype The Following GRIDS Were Generated and Are Available:

stype Directory: \%outdir\%slash $\%$ hucno\% $\%$ slash $\%$ grid

stype 1)mos\%hucno\%fl --> Filled-Sink DEM

\&type 2)mos\%hucno\%fd --> Flow-Direction GRID

stype 3) mos\%hucno\%fa --> Flow-Accumulation GRID

\&type 4)mos $\%$ hucno\%sl - -> Slope GRID (units in degrees)

\&type 5) mos\%hucno\%as - - Aspect GRID

stype 6)mos $\%$ hucno\%hs - -> Hillshade GRID

\&type 7) mos\%hucno\%h --> Hypsometric GRID

\&type 8)mos\%hucno\%ua --> Upslope Surface Contributing Area 


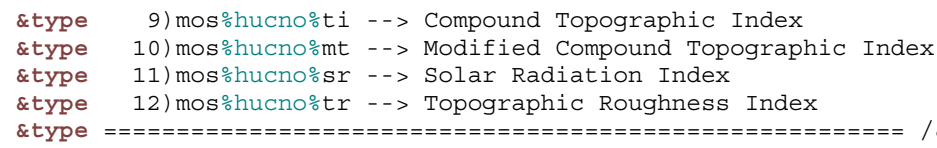

\&return

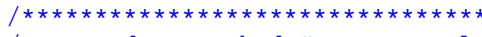

/* Forced Watershed GRID Removal

$/ * * * * * * * * * * * * * * * * * * * * * * * * * * * * * * * *$

\&routine wsgridclean

\&type $/ \& / \&$

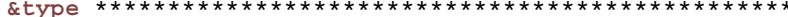

\&type The following routine will provide you with

\&type an interactive way to clean out all or specific

stype GRIDs that have been generated with this AML.

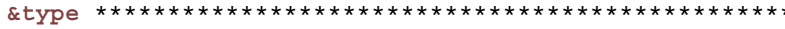

\&type $/ \& / \&$

\&sv continue [query 'Do you wish to continue [y $\mid \mathrm{n}]$ ' .FALSE.]

\&if $\%$ Continue $\%$. TRUE. \&then \&do

/*Verify that the provided HUC NO really exists \&call check

/*Check if user wants to flush everything or pick and choose

\&Sv all [query 'Do you want to flush out all GRIDs that were generated with watershed.aml

$[\mathrm{y} \mid \mathrm{n}]$ ' . FALSE]

\&if $\%$ all $\%=$.FALSE. \&then \&do

\&type /\& Cleaning out specific GRIDs.../\&

/*Check Filled GRID

\&if [exists \%outdir\%slash\%\%hucno\%slash\%grid\%slash\%mos\%hucno\%fl -grid] \&then \&do \&sv rmfl [query 'The Filled DEM exists, do you want to remove [y|n] '. FALSE]

\&if $\%$ rmfl\% $=$. TRUE. \&then \&do

\&type /\&Removing Filled GRID:

\%outdir\%:slash\%弓ucno\%:slash\%grid\%slash\%mos $\%$ hucno\%fl. . . \& send

kill \%outdir\%:slash\% \%ucno\%slash\%grid\%slash\%mos\%hucno\%fl all

\&end /*End checking Filled GRID

/*Check Flow Direction GRID

\&if [exists \%outdir\%:slash\%弓hucno\%slash\%grid\%slash\%mos\%hucno\%fd-grid] \&then \&do \&sv rmfd [query 'The Flow Direction GRID exists, do you want to remove [y|n] '. FALSE]

\&if $\%$ rmfdo $=$. TRUE. \&then \&do

\&type /\&Removing Flow Direction GRID:

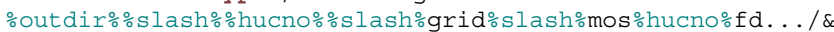

kill \%outdir\%:slash\%\%hucno\%:slash\%grid\%slash\%mos\%hucno\%fd all \&end

\&end /*End checking Flow Direction GRID

/*Check Flow Accumulation GRID

\&if [exists \%outdir\%:slash\%\%hucno\%slash\%grid\%slashomos\%hucno\%fa -grid] \&then \&do \&sv rmfa [query 'The Flow Accumulation GRID exists, do you want to remove [y|n] '.FALSE]

\&if $\frac{\circ}{\circ}$ rmfa $=$. TRUE. \&then \&do

stype /\&Removing Flow Accumulation GRID

\%outdir\%: \&end

kill \%outdir\%:slash\% $\%$ hucno\%:slash\%grid\%slash\%mos\%hucno\%fa all

\&end /*End checking Flow Accumulation GRID

/*Check Slope GRID

\&if [exists \%outdir\%:slasho\%hucno\%slashogridoslash\%mos\%hucno\%sl -grid] \&then \&do \&sv rmsl [query 'The Slope GRID exists, do you want to remove [y|n] '. FALSE]

\&if $\%$ rmsl\% $=$. TRUE. \&cthen \&do

\&type /\&Removing Slope GRID:

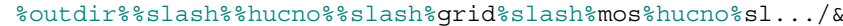

kill \%outdir\%:slash\%\%ucno\%slash\%grid\%slash\%mos\%hucno\%sl all \&cend

\&end /*End checking Slope GRID

/*Check Aspect GRID

\&if [exists \%outdir\%:slash\% $\%$ heno\%slash\%gridoslash\%mos\%hucno\%as -grid] \&then \&do \&sv rmas [query 'The Aspect GRID exists, do you want to remove [y|n] '. FALSE] 


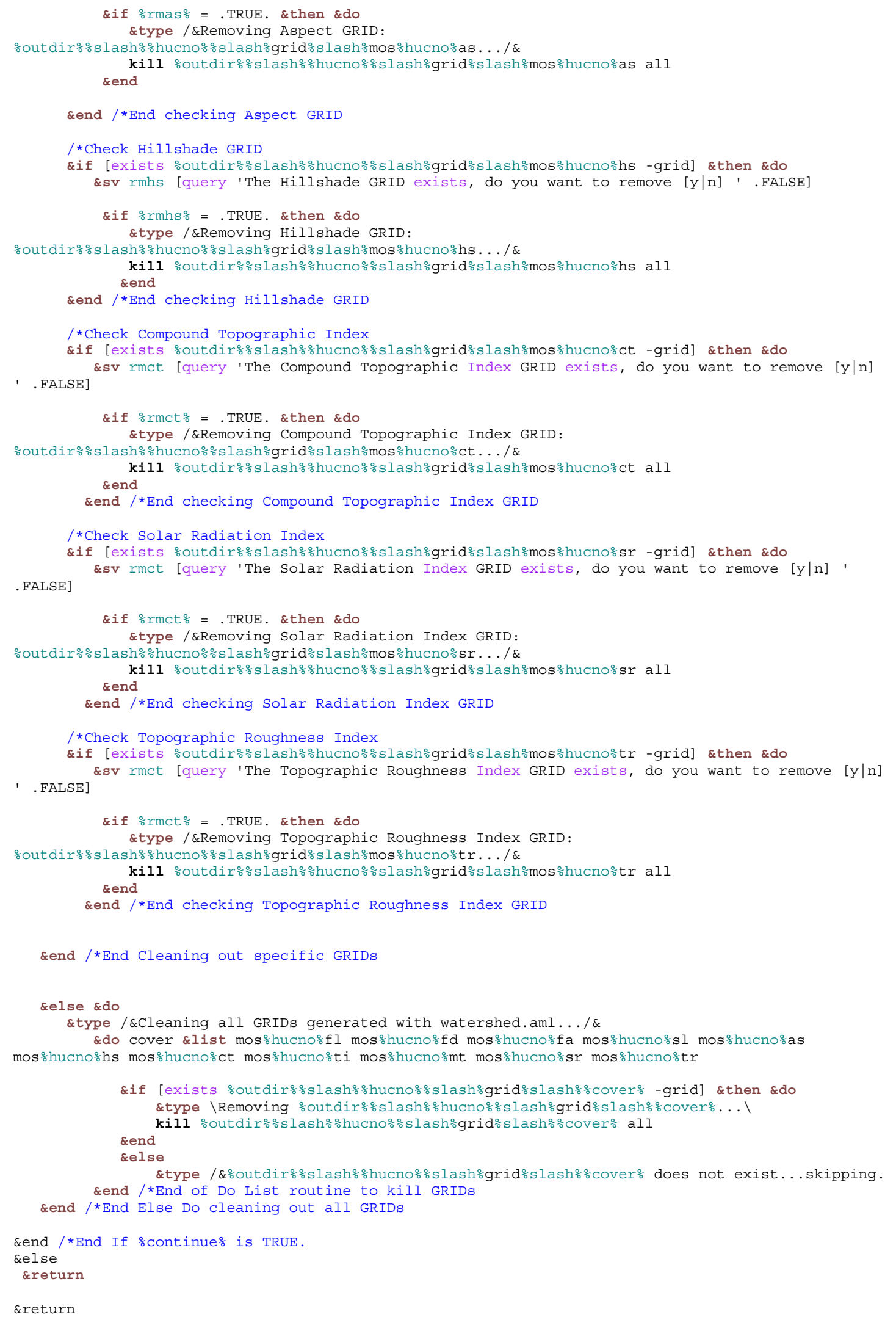




\title{
[ - watershed_saga.csh - ]
}

\author{
$\#$ !/bin/csh \\ \#\# SAGA Batch Script to Assemble Primary and Secondary Terrain Data \\ \#\# Batch File Assembled: Andre Coleman - 1 September 2006 \\ setenv SAGA_MLB/usr/saga/modules \\ set path $=\overline{(}$ \$path $/$ usr/saga / usr/saga/modules $)$ \\ echo \\ echo CALCULATING STANDARD TERRAIN ANALYSIS \\ saga_cmd ta_compound.so 0 -ELEVATION ./dem_fill.dgm -SHADE ./hillshade.dgm -SHADE_AZIM 160 - \\ SHADE DECL $\overline{4} 5$-SHADE EXAG 2.5 -SLOPE ./slope.dgm -ASPECT ./aspect.dgm - CURV ./curvature.dgm \\ HCURV - /hcurv.dgm -VCURV./vcurv.dgm -CONVERGENCE ./Convergence.dgm -CURVCLASS ./Curv class.dgm \\ -CAREA./catch_area.dgm -WETNESS./wetness.dgm -STREAMPOW ./strm_powr.dgm -LSFACTOR \\ ./ls_factor.dgm -CHNL_GRID./chanl_grd.dgm -CHNL_SHAPES ./chnl_shp.dgm -CHNL_INIT 5 -CHNL_ALTI \\ ./chñ__alt.dgm -CHNL_BASE./chnl_base.dgm -BASINS ./subbasins.ōgm \\ saga_cmd ta_morphometry.so 3 -ELEVATION ./dem.sgrd -SLOPE ./slope.sgrd -ASPECT ./aspect.sgrd - \\ HCURV ./hcurv.sgrd -VCURV ./vcurv.sgrd \\ saga_cmd ta_channels.so 0 -ELEVATION ./dem.sgrd -CAREA ./carea.sgrd \\ saga_cmd ta_indices.so 1 -SLOPE ./slope.sgrd -AREA ./carea.sgrd -WETNESS ./wetness.sgrd -STREAMPOW \\ ./streampow.sgrd -LSFACTOR. /lsfactor.sgrd \\ saga_cmd ta_lighting 0 -ELEVATION ./dem.sgrd -SHADE ./shade.sgrd -METHOD 0 -AZIMUTH -45 - \\ DECLINATION 45
}

exit 0 


\section{[ - strm_slp.aml - ]}

/** AML to Clip Stream Layers to Individual Subbasins and Extract Avg. Slope /** Coding: Andre Coleman

/* \&run strm_clp/projects/clearwater/ws_output strms10m 1 /projects/clearwater/ws_output

\&args source basin number output

\&if [null \%output:] \&then

\&return Usage: STRM_SLP <DATA SOURCE DIRECTORY> <STREAM COVER> <BASIN NUMBER> <OUTPUT DIRECTORY>

/** Set things up to run in UNIX or Windows by Modifying slashes

\&if [substr [extract 1 [show \&Os]] 1 10] = 'Windows NT' \&then

\&sv $\mathrm{slash}=1$

\&else

\&sv $\operatorname{slash}=/$

\&sv dem_base $=/$ projects $/$ clearwater $/$ grid $/$

\&call theme_clip

\&return

$/ * * * * * * * * * * x$

\&routine theme_clip

$1 * * * * * * * * * * * * * \bar{*} * * * * * * *$

\&sv out $=$ output $\%$ oslash $\%$ sb_ $\%$ number $\%$

/***Process Stream Data (vector data should be in data source directory) ***

$1 *$ Cleanup

\&if [exists oout\%:slash\%strm -cover] \&then

kill \%out\%:slashostrm all

\&if [exists $\%$ out $\%$ slash\%xxstrm -cover] \&then

kill out\%:

/*Clip Stream Vectors to Basin Boundary

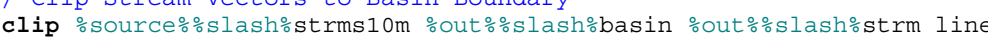

/*Determine Number of Stream Segments

\&work \%out:

display 0

arcedit

display 0

ec strm

ef line

sel all

\&sv strmsel $=[$ extract 1 [show number select] $]$

/*If there is only one stream segment

\&if $\%$ strmsel\% $=1$ \&then \&do

put xxstrm

quit /*Out of ArcEdit

\&end

/*If there are multiple stream segments

\&if $\%$ strmsel\% gt 1 \&then \&do

quit /*Out of ArcEdit

copy strm xxstrm

build xxstrm line

tables

sel xxstrm.aat

sort length (D)

\&SV strmid $=$ [SHOW RECORD 1 ITEM XXSTRM-ID]

quit /*Tables

kill xxstrm all

display 0

arcedit

display 0

ec strm

ef line

sel strm-id = \%strmid

put xxstrm

quit /*Out of ArcEdit

build xxstrm line

send

/*If there are no stream segments

\&if $\%$ strmsel\% $=0$ \&then \&do

quit /*Out of ArcEdit 
additem basin.pat basin.pat strm slope $77 \mathrm{~N} 3$

tables

sel basin.pat

calc strm_slope $=-1$

quit /*out of Tables

\&work .././scripts

sreturn

\&end

/ Convert stream segment end-nodes into points

nodepoint xxstrm xxstrm pt

build xxstrm_pt point

*Extract Elevation Values

latticespot \%dem base $\%$ slash\%mostul xxstrm_pt elev

/*Sort and Record Min/Max Elevation Values

tables

sel xxstrm_pt.pat

sort elev

\&Sv minelev $=[$ SHOW RECORD 1 ITEM ELEV]

$\&$ Sv maxelev $=[$ SHOW RECORD 2 ITEM ELEV]

/*Determine Stream Segment Length

sel xxstrm.aat

\&sv strmlength $=$ [SHOW RECORD 1 ITEM LENGTH]

/*Calculate Average Stream Gradient

\&sv delta elev $=[$ calc \%maxelev\% - \%minelevo] $/ *$ Rise

\&sv slope dec $=[\mathrm{calc} \%$ delta elev\% / \%strmlength\%]

\&sv slope perc $=\left[\right.$ calc $\%$ slope dec $\left.^{\circ} * 100\right] / *$ Percent slope

quit /*Out of Tables

/*Additem to Basin Coverage

\&sv itemexist $=[$ iteminfo basin -poly STRM SLOPE -exists]

\&if \%itemexist\% = .FALSE. \&then

additem basin.pat basin.pat strm slope $77 \mathrm{~N} 3$

\section{tables}

sel basin.pat

calc strm_slope $=\circ$ slope_perc $\%$

quit /*Out of Tables

/*Cleanup

kill xxstrm all

kill xxstrm_pt all

/*Return to Scripts Directory

w ..././scripts

\&return 


\section{[ - solarcalc.csh - ]}

$\#$ !/bin/csh

\#\# SAGA Batch Script to Assemble Daily Solar and Insolation Properties

\#\# Batch File Assembled: Andre Coleman 16 August 2006

\#\#

setenv SAGA_MLB/usr/saga/modules

set path $=$ ( $\$$ path / usr/saga / usr/saga/modules $)$

\#Setup Variables

setenv elev dem10m_dg.dgm

setenv solconst $13 \bar{\sigma} \overline{7}$

setenv transmitt 70

setenv pressure 1013

setenv water 1.68

setenv dust 100

setenv latitude 47.89

echo

echo Calculating Solar Radiation and Insolation for January

foreach d in $\left(\begin{array}{lllllllllllllllllllllllllllllll}0 & 1 & 3 & 4 & 5 & 6 & 7 & 8 & 9 & 10 & 11 & 12 & 13 & 14 & 15 & 16 & 17 & 18 & 19 & 20 & 21 & 22 & 23 & 24 & 25 & 26 & 27 & 28 & 29 & 30\end{array}\right)$ saga_cmd ta_lighting.so 2 -ELEVATION ./\$elev -INSOLAT ./jan/inso_d.dgm -DURATION ./jan/durat_d.dgm -UNIT 0 -SOLCONST \$solconst -METHOD 0 -TRANSMITT \$transmitt -PRESSURE \$pressure -WATER \$water DUST \$dust -LATITUDE \$latitude -HOUR_RANGE_MIN 4 -HOUR_RANGE_MAX 23 -HOUR_STEP 1 -TIMESPAN 0 SINGLE_DAY_DAY d -SINGLE_DAY_MONTH O end

echo

echo Calculating Solar Radiation and Insolation for February

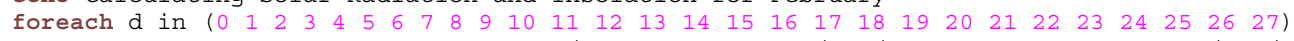
saga_cmd ta_lighting.so 2 -ELEVATION./\$elev -INSOLAT./feb/inso_d.dgm -DURATION ./feb/durat_d.dgm -UNIT 0 -SOL̈CONST \$solconst -METHOD 0 -TRANSMITT \$transmitt -PRESSURE \$pressure -WATER \$water DUST \$dust -LATITUDE \$latitude -HOUR_RANGE_MIN 4 -HOUR_RANGE_MAX 23 -HOUR_STEP 1 -TIMESPAN 0 SINGLE DAY DAY d -SINGLE DAY MONTH 1 end

echo

echo Calculating Solar Radiation and Insolation for March

foreach d in $\left(\begin{array}{llllllllllllllllllllllllllllllll}0 & 1 & 2 & 3 & 4 & 5 & 6 & 7 & 8 & 9 & 10 & 11 & 12 & 13 & 14 & 15 & 16 & 17 & 18 & 19 & 20 & 21 & 22 & 23 & 24 & 25 & 26 & 27 & 28 & 29 & 30\end{array}\right)$ saga_cmd ta_lighting.so 2 -ELEVATION./\$elev -INSOLAT ./mar/inso_d.dgm -DURATION ./mar/durat_d.dgm -UNIT 0 -SOLCONST \$solconst -METHOD 0 -TRANSMITT \$transmitt -PRESSURE \$pressure -WATER \$water DUST \$dust -LATITUDE \$latitude -HOUR_RANGE_MIN 4 -HOUR_RANGE MAX 23 -HOUR_STEP 1 -TIMESPAN 0 SINGLE_DAY_DAY d -SINGLE_DAY_MONTH 2

end

echo

echo Calculating Solar Radiation and Insolation for April

foreach d in $\left(\begin{array}{lllllllllllllllllllllllllllllll}0 & 1 & 2 & 3 & 4 & 5 & 6 & 7 & 8 & 9 & 10 & 11 & 12 & 13 & 14 & 15 & 16 & 17 & 18 & 19 & 20 & 21 & 22 & 23 & 24 & 25 & 26 & 27 & 28 & 29\end{array}\right)$ saga_cmd ta_lighting.so 2 -ELEVATION ./\$elev -INSOLAT ./apr/inso_d.dgm -DURATION ./apr/durat_d.dgm -UNIT 0 -SOLCONST \$solconst -METHOD 0 -TRANSMITT \$transmitt -PRESSURE \$pressure -WATER \$water DUST \$dust -LATITUDE \$latitude -HOUR_RANGE_MIN 4 -HOUR_RANGE_MAX 23 -HOUR_STEP 1 -TIMESPAN 0 SINGLE_DAY_DAY d -SINGLE_DAY_MONTH 3

end

echo

echo Calculating Solar Radiation and Insolation for May

foreach d in $\left(\begin{array}{llllllllllllllllllllllllllllllll}0 & 1 & 2 & 3 & 4 & 5 & 6 & 7 & 8 & 9 & 10 & 11 & 12 & 13 & 14 & 15 & 16 & 17 & 18 & 19 & 20 & 21 & 22 & 23 & 24 & 25 & 26 & 27 & 28 & 29 & 30\end{array}\right)$

saga cmd ta lighting.so 2 -ELEVATION./\$elev-INSOLAT./may/inso d.dgm -DURATION ./may/durat d.dgm -UNIT 0 -SOLCONST \$solconst -METHOD 0 -TRANSMITT \$transmitt -PRESSURE \$pressure -WATER \$water DUST \$dust -LATITUDE \$latitude -HOUR_RANGE_MIN 4 -HOUR_RANGE_MAX 23 -HOUR_STEP 1 -TIMESPAN 0 SINGLE_DAY_DAY d -SINGLE_DAY_MONTH 4

end

echo

echo Calculating Solar Radiation and Insolation for June

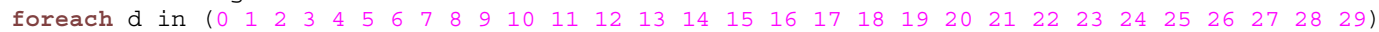
saga cmd ta lighting.so 2 -ELEVATION ./\$elev -INSOLAT ./jun/inso d.dgm -DURATION ./jun/durat_d.dgm -UNIT 0 -SOL̈CONST \$SOlconst -METHOD 0 -TRANSMITT \$transmitt -PRESSSURE \$pressure -WATER \$water DUST \$dust - LATITUDE \$latitude -HOUR_RANGE_MIN 4 -HOUR_RANGE_MAX 23 -HOUR_STEP 1 -TIMESPAN 0 SINGLE_DAY_DAY d -SINGLE_DAY_MONTH 5

end

echo

echo Calculating Solar Radiation and Insolation for July

foreach d in $\left(\begin{array}{lllllllllllllllllllllllllllllll}0 & 1 & 3 & 4 & 5 & 6 & 7 & 8 & 9 & 10 & 11 & 12 & 13 & 14 & 15 & 16 & 17 & 18 & 19 & 20 & 21 & 22 & 23 & 24 & 25 & 26 & 27 & 28 & 29 & 30\end{array}\right)$ saga_cmd ta_lighting.so 2 -ELEVATION ./\$elev -INSOLAT ./jul/inso_d.dgm -DURATION ./jul/durat_d.dgm -UNIT 0 -SOLCONST \$solconst -METHOD 0 -TRANSMITT \$transmitt -PRESSURE \$pressure -WATER \$water DUST \$dust -LATITUDE \$latitude -HOUR_RANGE_MIN 4 -HOUR_RANGE_MAX 23 -HOUR_STEP 1 -TIMESPAN 0 SINGLE_DAY_DAY d -SINGLE_DAY_MONTH 6 end

echo echo Calculating Solar Radiation and Insolation for August 


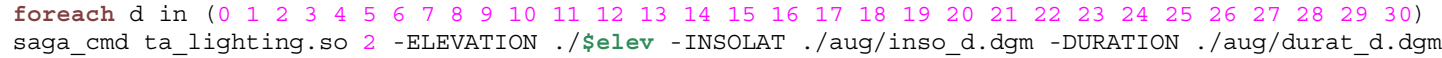
-UNIT 0 -SOLCONST \$solconst -METHOD 0 -TRANSMITT \$transmitt -PRESSURE \$pressure -WATER \$water DUST \$dust - LATITUDE \$latitude -HOUR_RANGE_MIN 4 -HOUR_RANGE_MAX 23 -HOUR_STEP 1 -TIMESPAN 0 SINGLE_DAY_DAY d -SINGLE_DAY_MONTH 7

end

echo

echo Calculating Solar Radiation and Insolation for September

foreach d in $\left(\begin{array}{llllllllllllllllllllllllllllll}0 & 1 & 2 & 3 & 4 & 5 & 6 & 7 & 8 & 9 & 10 & 11 & 12 & 13 & 14 & 15 & 16 & 17 & 18 & 19 & 20 & 21 & 22 & 23 & 24 & 25 & 26 & 27 & 28 & 29\end{array}\right)$

saga cmd ta lighting.so 2 -ELEVATION./\$elev -INSOLAT ./sep/inso d.dgm -DURATION ./sep/durat_d.dgm -UNIT 0 -SOLECONST \$solconst -METHOD 0 -TRANSMITT \$transmitt -PRESSURE \$pressure -WATER \$water DUST \$dust -LATITUDE \$latitude -HOUR_RANGE_MIN 4 -HOUR_RANGE_MAX 23 -HOUR_STEP 1 -TIMESPAN 0 SINGLE_DAY_DAY d -SINGLE_DAY_MONTH 8

end

echo

echo Calculating Solar Radiation and Insolation for October

foreach $d$ in $\left(\begin{array}{lllllllllllllllllllllllllllllll}0 & 1 & 2 & 3 & 4 & 5 & 6 & 7 & 8 & 9 & 10 & 11 & 12 & 13 & 14 & 15 & 16 & 17 & 18 & 19 & 20 & 21 & 22 & 23 & 24 & 25 & 26 & 27 & 28 & 29 & 30\end{array}\right)$ saga_cmd ta_lighting.so 2 -ELEVATION./\$elev -INSOLAT ./oct/inso_d.dgm -DURATION ./oct/durat_d.dgm -UNIT 0 -SOLLCONST \$SOlconst -METHOD 0 -TRANSMITT \$transmitt -PRESSURE \$pressure -WATER \$water DUST \$dust -LATITUDE \$latitude -HOUR_RANGE_MIN 4 -HOUR_RANGE_MAX 23 -HOUR_STEP 1 -TIMESPAN 0 SINGLE_DAY_DAY d -SINGLE_DAY_MONTH 9

end

\section{echo}

echo Calculating Solar Radiation and Insolation for November

foreach d in $\left(\begin{array}{lllllllllllllllllllllllllllll}0 & 1 & 2 & 3 & 4 & 6 & 7 & 8 & 10 & 11 & 12 & 13 & 14 & 15 & 16 & 17 & 18 & 19 & 20 & 21 & 22 & 23 & 24 & 25 & 26 & 27 & 28 & 29\end{array}\right)$ saga_cmd ta_lighting.so 2 -ELEVATION ./\$elev -INSOLAT ./nov/inso_d.dgm -DURATION ./nov/durat_d.dgm -UNIT 0 -SOLCONST \$SOlconst -METHOD 0 -TRANSMITT \$transmitt -PRESSURE \$pressure -WATER \$water DUST \$dust -LATITUDE \$latitude -HOUR_RANGE_MIN 4 -HOUR_RANGE_MAX 23 -HOUR_STEP 1 -TIMESPAN 0 SINGLE_DAY_DAY d -SINGLE_DAY_MONTH $1 \overline{0}$ end

\section{echo}

echo Calculating Solar Radiation and Insolation for December

foreach d in $\left(\begin{array}{llllllllllllllllllllllllllllll}0 & 1 & 3 & 4 & 5 & 6 & 7 & 8 & 9 & 10 & 11 & 12 & 13 & 14 & 15 & 16 & 17 & 18 & 19 & 20 & 21 & 22 & 23 & 24 & 25 & 26 & 27 & 28 & 29 & 30\end{array}\right)$ saga_cmd ta_lighting.so 2 -ELEVATION ./\$elev -INSOLAT./dec/inso_d.dgm -DURATION ./dec/durat_d.dgm -UNIT 0 -SOLCONST \$SOlconst -METHOD 0 -TRANSMITT \$transmitt -PRESSURE \$pressure -WATER \$water DUST \$dust -LATITUDE \$latitude -HOUR RANGE MIN 4 -HOUR RANGE MAX 23 -HOUR STEP 1 -TIMESPAN 0 SINGLE_DAY_DAY d -SINGLE_DAY_MONTH $1 \overline{1}$ end

exit 0 


\section{[ - dump_sb_values.aml - ]}

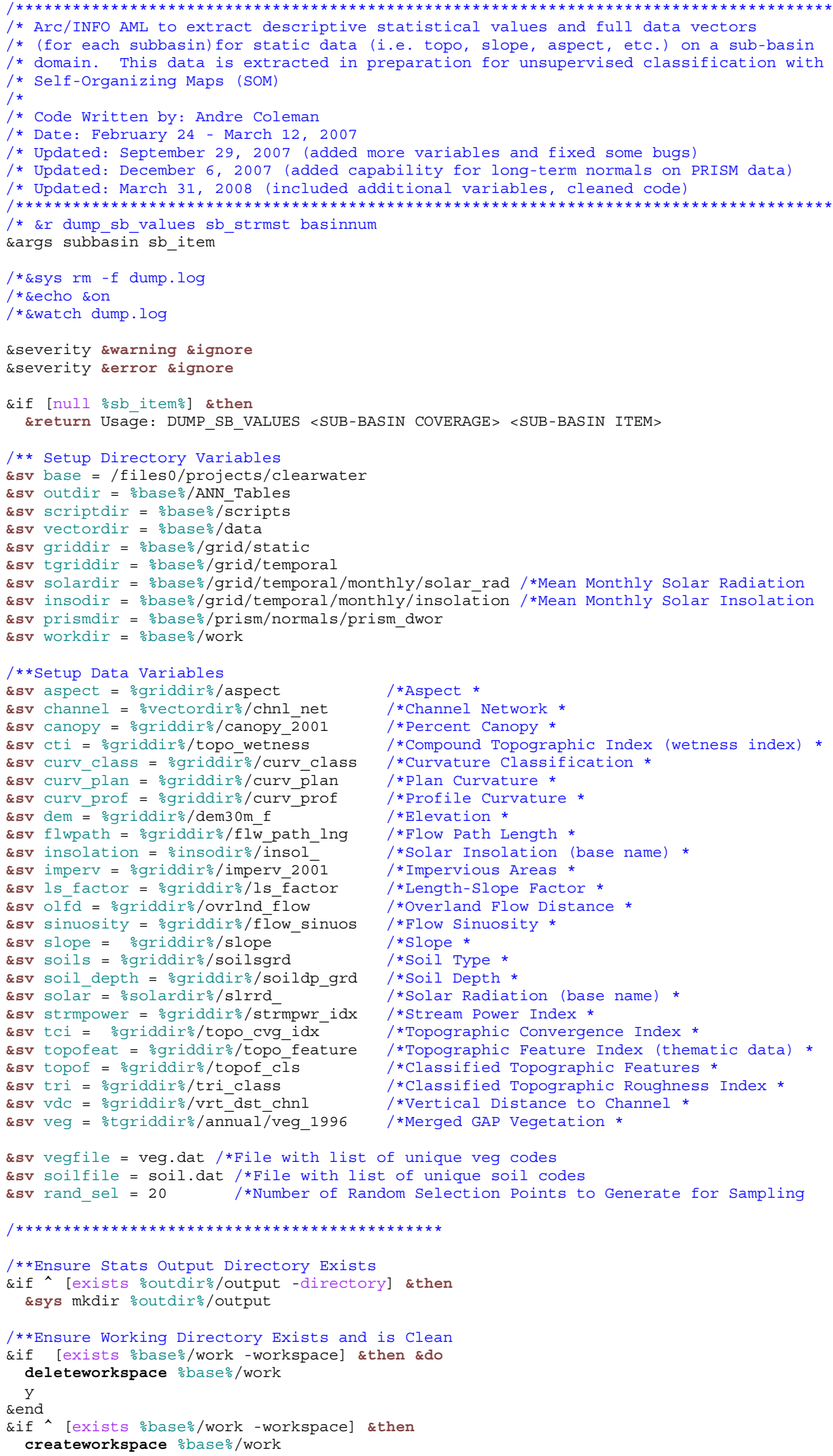




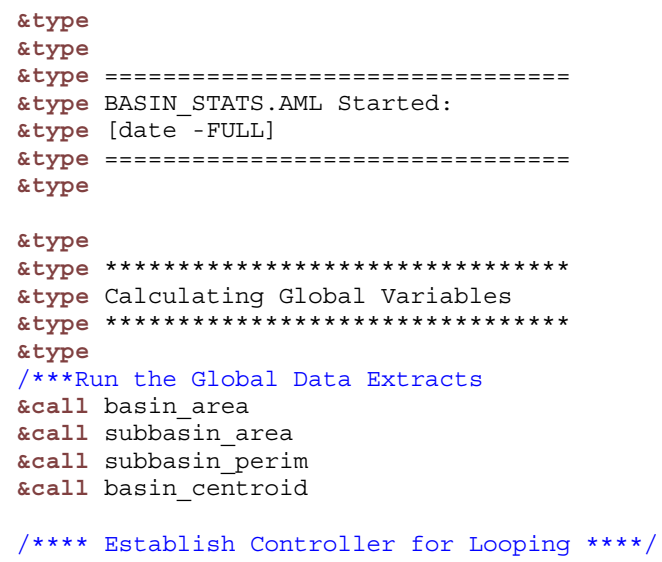

/*Determine Number of Subbasins to Process \&describe \%vectordir\%/\%subbasin\% \&SV num_inst $=$ DSC\$POLYGONS\%

$\& \mathrm{sv}$ count $=1$

\&do suntil \%count\% gt \%num_inst:

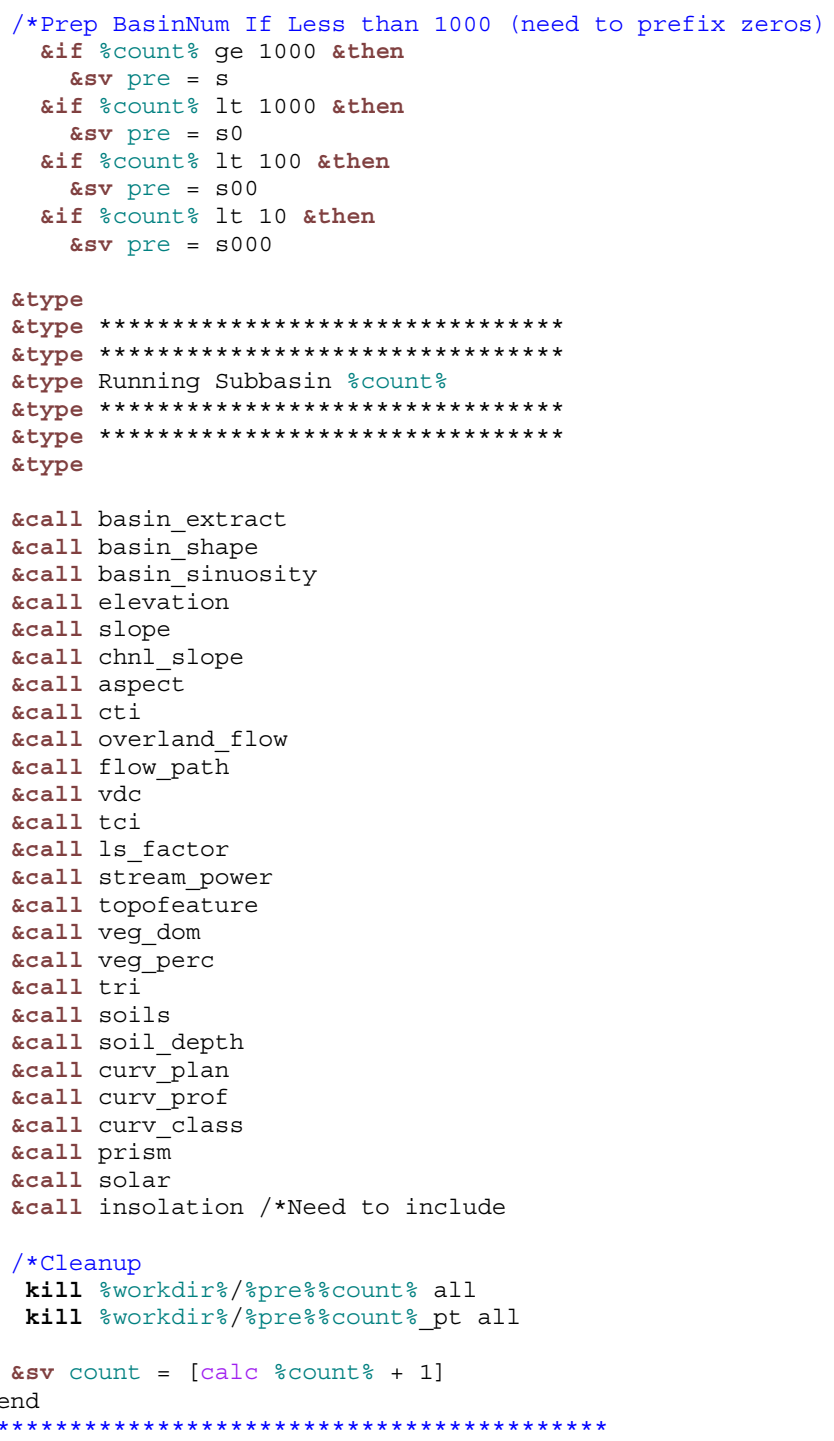

\&return 


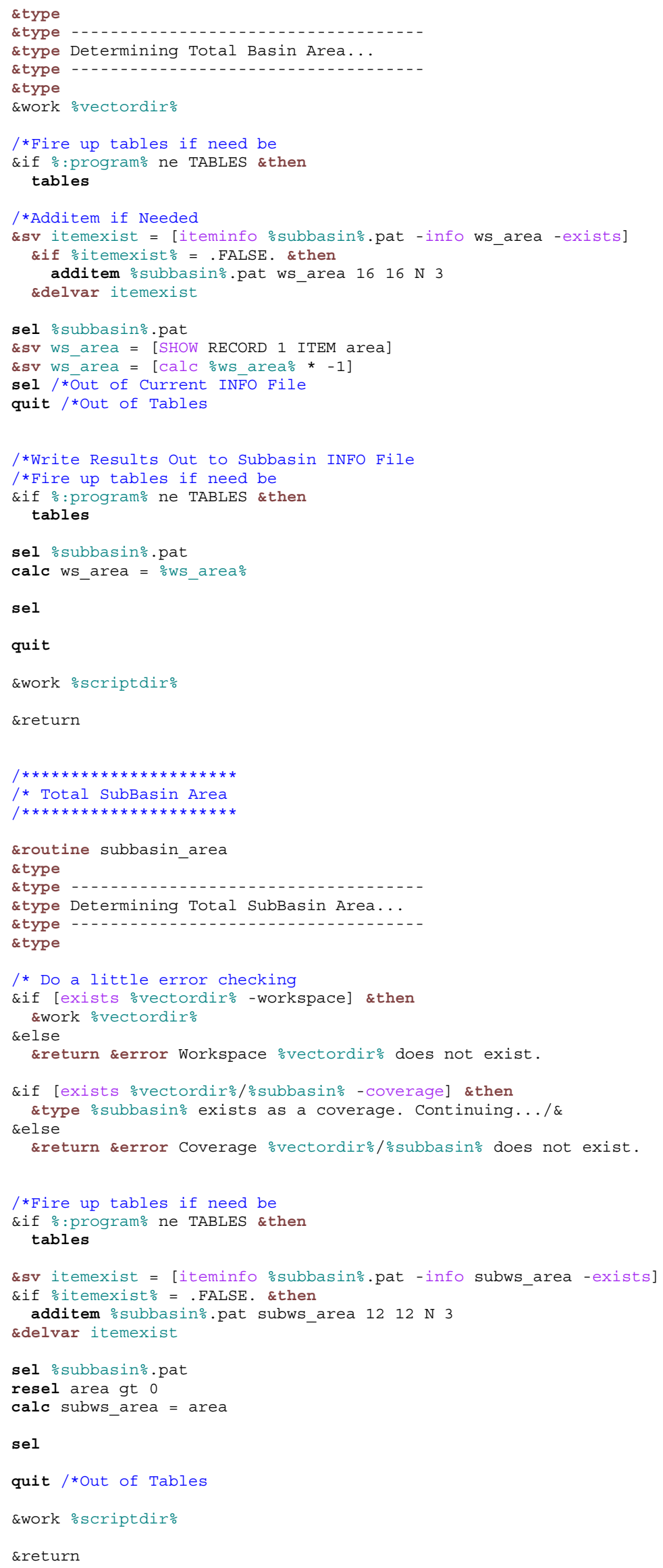




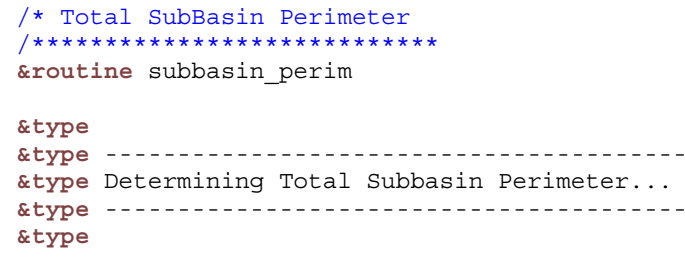

\&work vectordir\%

/*Fire up tables if need be \&if :program\% ne TABLES \&then

\section{tables}

\&sv itemexist $=$ [iteminfo $\%$ subbasin\%.pat -info subws_perim -exists]

\&if $\%$ itemexist $=$.FALSE. \&then

additem \%subbasin\%.pat subws_perim $1212 \mathrm{~N} 3$

\&delvar itemexist

sel \%subbasin\%.pat

calc subws_perim = perimeter

sel

quit /*Out of Tables

\&work \%scriptdir\%

\&return

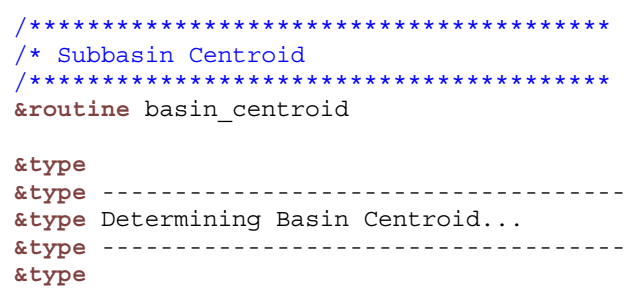

tables

\&sv itemexist $=$ [iteminfo $\%$ subbasin\%.pat -info UTMX -exists]

\&if \%itemexist $=$.FALSE. \&then

additem \%subbasin\%.pat UTMX $1212 \mathrm{~N} 3$

\&delvar itemexist

\&sv itemexist $=$ [iteminfo $\frac{\circ}{\text { subbasin }}$.pat -info UTMY -exists]

\&if $\%$ itemexist $=$. FALSE. \&then

additem \%subbasin\%.pat UTMY $1212 \mathrm{~N} 3$

\&delvar itemexist

sel basin ctr.pat

/*Extract UTM Coords to Variables \&sv utmX $=$ [SHOW RECORD 1 ITEM $\mathrm{X}$-coord]

\&sv utmy $=$ [SHOW RECORD 1 ITEM $\mathrm{y}$-coord]

/*Write Variables to Basin Coverage

sel \%subbasin\%.pat

calc utmx = outmx

calc utmy $=$ outmy 
quit /*out of Tables

/*Project Basin Centroid to a Geographic Coordinate \&if [exists basin ctrl-cover] \&then

kill basin ctrl all

project cover basin_ctr basin_ctrl \%base\%/prj/utmllnad83_dd83.prj

dropitem basin_ctrl.pat basin_ctrl.pat $\mathrm{x}$-coord $\mathrm{y}$-coord

additem basin ctrl.pat basin ctrl.pat x-coord $812 \mathrm{~F} 6$

additem basin ctrl.pat basin ctrl.pat y-coord 812 F 6

addxy basin_ctrr

tables

\&sv itemexist $=$ [iteminfo \%subbasin\%.pat -info LAT -exists $]$

\&if $\%$ itemexist $=$. FALSE. \& then

additem $\%$ subbasin:pat LAT $1212 \mathrm{~N} 6$

\&delvar itemexist

\&Sv itemexist $=$ [iteminfo $\div$ subbasin ,pat - info LONG - exists $]$

\&if \%itemexist $=$. FALSE. \&then

additem \%subbasin\%.pat LONG $1212 \mathrm{~N} 6$

\&delvar itemexist

sel basin_ctrl.pat

/*Extract Geographic Coords to Variables

\&sv lat $=$ [SHOW RECORD 1 ITEM $\mathrm{y}$-coord]

\&SV long $=$ [SHOW RECORD 1 ITEM $\mathrm{x}$-coord]

/*Write Variables to Basin Coverage

sel \%subbasin\%.pat

calc lat $=\%$ lat

calc long $=\frac{\circ}{\circ}$ long

sel

quit /*Out of Tables

/* Cleanup

\&if [exists ovectordir\%/basin_ctr -cover] \&then

kill \%vectordir\%/basin_ctr āll

\&work \%scriptdir\%

\&return

/* Basin Extract (pull out individual sub-basin)

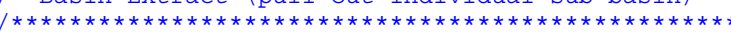

\&routine basin_extract

\&type

stype -...............................

\&type Pulling Out Individual Sub-Basin...

\&type -...............

stype

\&work \%vectordir\%

/*Check for Existing Files First

\&if [exists $\%$ workdir\%/\%pre\% $\%$ count $\%$-cover] \&then

kill $\%$ workdir\%/\%pre $\%$ Count $\%$ all

/*Start ArcEdit and Pull out Polygon

display 0

arcedit

display 0

ec $\frac{\circ}{5}$ ubbasin:

ef poly

sel $\%$ sb item $\%=$ count $\%$

put \%workdir\%/\%pre\%count

ef point

sel $\%$ sb item $\%$ count

put $\%$ workdir\%/\%pre $\%$ count $\%$ pt

quit

build \%workdir\%/\%pre $\%$ count

\&work \%scriptdir\% 
\&return

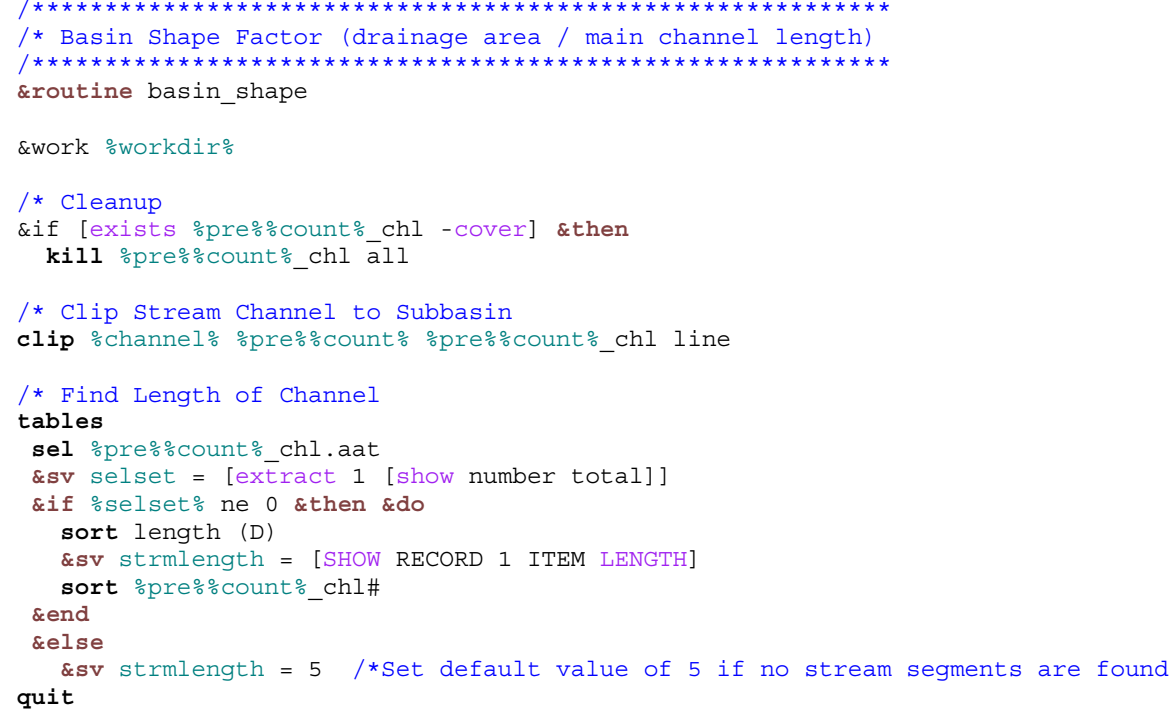


\&Sv $\operatorname{sinu}$ sd $=\%$ GRD $\$ S T D V \%$

/*Remove Data

kill $\%$ pre\%count\%_sin all

/*Additems to Basin Coverage

\&work \%vectordir\%

tables

\&sv itemexist $=[$ iteminfo $\%$ subbasino pat - info sinu_min - exists $]$

\&if $\%$ itemexist $=$.FALSE. \&then

additem $\%$ subbasin\%.pat sinu min $88 \mathrm{~N} 3$

\&delvar itemexist

\&sv itemexist $=[$ iteminfo $\%$ subbasin $\%$ pat - info sinu_max - exists $]$

\&if \%itemexist $=$.FALSE. \&then

additem $\%$ subbasin $\%$ pat sinu $\max 88 \mathrm{~N} 3$

\&delvar itemexist

\&sv itemexist $=[$ iteminfo $\%$ subbasin $\%$ pat - info sinu_mean - exists $]$

\&if \%itemexist $=$.FALSE. \&then

additem $\%$ subbasin\%. pat sinu mean $88 \mathrm{~N} 3$

\&delvar itemexist

\&sv itemexist $=$ [iteminfo $\%$ subbasin\%.pat -info sinu_sd -exists]

\&if \%itemexist $=$.FALSE. \&then

additem $\%$ subbasin $\%$ pat sinu sd $88 \mathrm{~N} 3$

\&delvar itemexist

sel \%subbasin\%.pat

sel \%subbasin\%.pat

resel $\%$ sb item $\%$ = count $\%$

calc sinu_min $=\%$ sinu min

calc sinu_max $=\%$ sinu_max

calc sinu mean $=\%$ sinu mean $\%$

calc sinu_sd $=\frac{\circ}{5}$ sinu_sdo

sel

quit /*Out of Tables

\&work 을iptdir\%

\&return

$/ * * * * * * * * * * * * * * * * * * * *$

\&routine elevation

$1 * * * * * * * * * * * * * * * * * * * *$

\&type

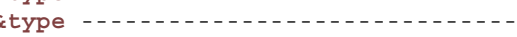

\&type Calculating Elevation Stats...

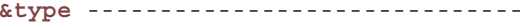

stype

\&work \%workdir\%

$/ *$ Cleanup

\&if [exists \%workdir\%/\%pre\%count\% dem -grid] \&then

kill \%workdir\%/\%pre\%count\% dem āll

\&if [exists $\%$ workdir\%/\%pre\%count $\%$ dem_p -cover] \& then

kill \%workdir\%/\%pre\% \%ount\%_dem_p al

/*Clip Elevation Data to Subbasin

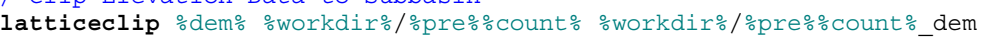

/**** Calculate Summary Statistics to Apply Back to Master Subbasin Coverage **** \&describe \%workdir\%/\%pre\%count\%_dem

\&sv elev_min $=$ \%GRD\$ZMIN\%

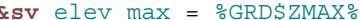

\&SV elev mean = $\circ$ GRD \$MEAN

\&sv elev sd $=\%$ GRD $\$$ STDV

\&sv elev_delta $=[$ calc $\%$ elev_max\% - \%elev_min\%]

*Additems to Basin Coverage

\&work \%vectordir\%

tables 


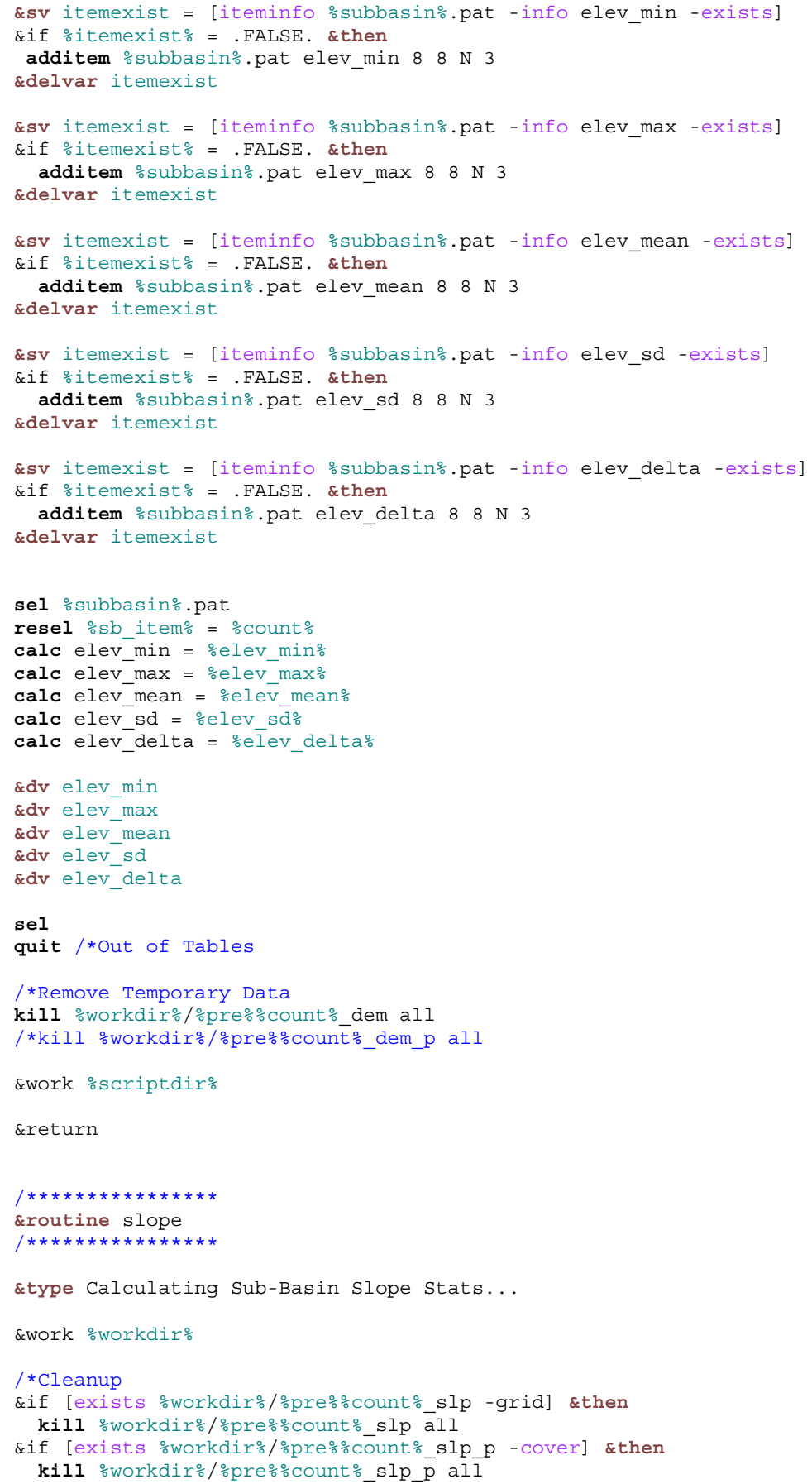




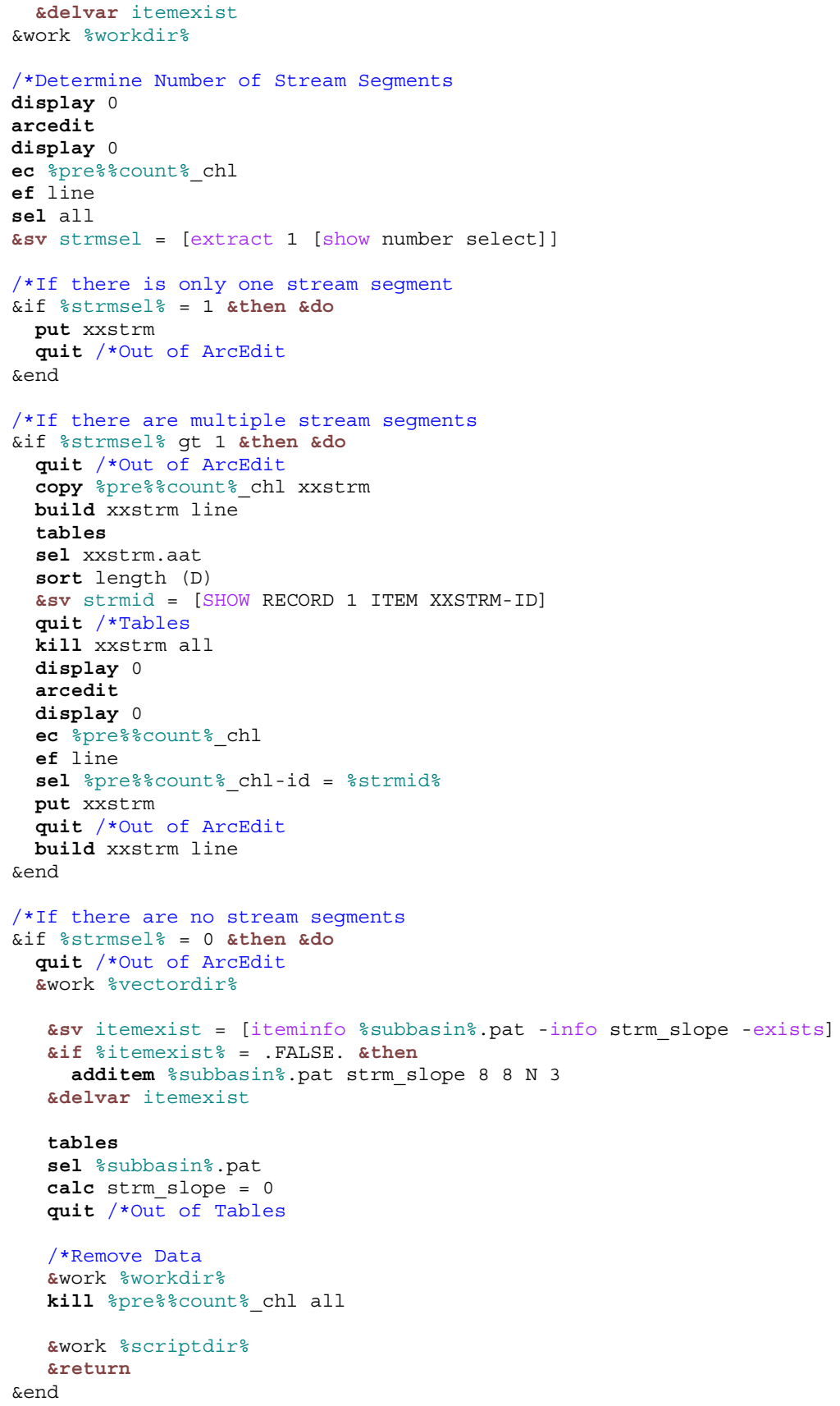

/*Convert stream segment end-nodes into points nodepoint xxstrm xxstrm_pt build xxstrm_pt point

/*Extract Elevation Values

latticespot $\%$ dem\% xxstrm_pt elev

/*Sort and Record Min/Max Elevation Values tables

sel xxstrm_pt.pat

sort elev

\&sv minelev $=[$ SHOW RECORD 1 ITEM ELEV]

\&sv maxelev $=[$ SHOW RECORD 2 ITEM ELEV]

/*Determine Stream Segment Length

sel xxstrm.aat

\&Sv strmlength $=$ [SHOW RECORD 1 ITEM LENGTH]

/*Calculate Average Stream Gradient

\&sv delta elev $=[\mathrm{calc} \%$ maxelev\% - \%minelev\%] $/ *$ Rise

\&sv slope dec $=[$ calc $\%$ delta elevo / \%strmlength\%]

\&sv slope_perc $=[$ calc oslope_dec $\%$ * 100] / Percent slope 
quit /*out of Tables

\&work \%vectordir\%

\&sv itemexist $=$ [iteminfo $\%$ subbasin\%.pat - info strm slope -exists]

\&if \%itemexist\% $=$.FALSE. \&then

additem $\%$ subbasin\%.pat \%subbasin\%,pat strm_slope $88 \mathrm{~N} 3$

\&delvar itemexist

\section{tables}

sel \%subbasin\%.pat

resel $\%$ sb_item\% $=\%$ count $\%$

calc strm_slope $=\%$ slope perco

quit /*out of Tables

/*Remove Data

\&work \%workdir:

kill xxstrm all

kill xxstrm pt all

kill \%pre\% $\%$ count\%_chl all

\&work ㅇriptdir\%

\&return

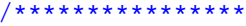

\&routine aspect

$1 * * * * * * * * * * * * * * * *$

\&type Calculating Sub-Basin Aspect Stats...

\&work \%workdir\%

$1 *$ Cleanup

\&if [exists \%workdir\%/\%pre\% count\%_asp -grid] \&then

kill \%workdir\%/\%pre\% count $\%$ asp all

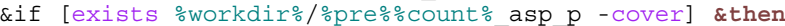

kill \%workdir\%/\%pre\%

*Clip Elevation Data to Subbasin

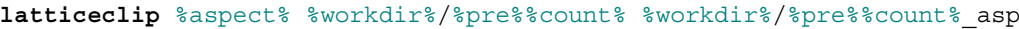

\&describe $\%$ pre $\%$ count

$\&$ Sv aspect $\min =\circ G R D \$ Z M I N \%$

$\& \mathrm{SV}$ aspect $\max =\% \mathrm{GRD} \$ \mathrm{ZMAX} \div$

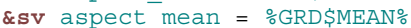

\&sV aspect_sd $=\% \mathrm{GRD} \$ \mathrm{STDV}$

/*** Calculate Percentage of Cells with North and South Facing Slopes $1 *$ Convert to Points

gridpoint \%workdir\%/\%pre \% Count

* How Many Points?

\&describe \%workdir\%/\%pre\%count: asp_pt

\&sv npoints $=$ DDSC\$POINTS\%

\&work \%workdir\%

tables

sel $\%$ pre $\%$ count $\%$ asp_pt.pat

$1 *$ North Slopes

resel aspect le 45

asel aspect ge 315

\&sv northsel $=$ [extract 1 [show number select]

asel

* South Slopes

resel aspect ge 135 AND aspect le 225

\&sv southsel $=$ [extract 1 [show number select]]

quit

\&sv northslp perc $=[$ calc $[$ calc onorthsel\% / \%npoints\%] * 100]

\&sv southslp_perc $=[$ calc $[$ calc $\%$ southsel\% $/$ onpoints\%] * 100]

$1 * * *$

\&work vectordir\%

/*Additems to Basin Coverage

tables

sel $\%$ subbasin $\cdot$ pat

\&sv itemexist $=$ [iteminfo $\%$ subbasin\%.pat -info aspect_min -exists] 
\&if $\%$ itemexist $\%$.FALSE. \&then

additem $\div$ subbasin. pat aspect_min $88 \mathrm{~N} 3$

\&delvar itemexist

\&sv itemexist $=$ [iteminfo $\%$ subbasin\%.pat -info aspect max -exists]

\&if \%itemexist $=$.FALSE. \&then

additem $\%$ subbasin\%.pat aspect_max $88 \mathrm{~N} 3$

\&delvar itemexist

\&sv itemexist $=$ [iteminfo \%subbasin\%.pat -info aspect mean - exists]

\&if $\%$ itemexist $\%$.FALSE. \&then

additem \%subbasin\%.pat aspect_mean $88 \mathrm{~N} 3$

\&delvar itemexist

\&sv itemexist $=$ [iteminfo $\%$ subbasin\%.pat - info aspect sd -exists

\&if \%itemexist $=$. FALSE. \&then

additem $\%$ subbasin\%.pat aspect_sd $88 \mathrm{~N} 3$

\&delvar itemexist

\&sv itemexist $=$ [iteminfo $\%$ subbasin\%.pat -info north perc -exists]

\&if \%itemexist: = .FALSE. \&then

additem \%subbasin\%.pat north_perc $88 \mathrm{~N} 3$

\&delvar itemexist

\&sv itemexist $=[$ iteminfo $\%$ subbasin. pat - info south perc -exists $]$

\&if \%itemexist $=$.FALSE. \& then

additem $\%$ subbasin\%.pat south_perc $88 \mathrm{~N} 3$

\&delvar itemexist

sel $\%$ subbasin\%.pat

resel $\%$ sb_item $\%$ 응 count $\%$

calc aspect_min $=$ \%aspect_min\%

calc aspect $\max =\frac{\circ}{0}$ aspect $\max \%$

calc aspect mean = \%aspect mean:

calc aspect_sd $=\%$ aspect_s $\overline{\mathrm{d}} \%$

calc north_perc $=$ onorthslp_perc\%

calc south_perc $=$ osouthslp_perco

sel

quit /*Out of Tables

/*Remove Temporary Data

kill \%workdir\%/\%pre\%ㅇount\%_asp all

kill \%workdir\%/\%pre\%count\%_asp_pt all

\&work $\frac{\circ}{5}$ scriptdir\%

\&return

(**********************

\&routine cti

$* * * * * * * * * * * * * * * * * * * *$

\&type

type

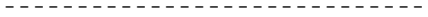

\&type Calculating Compound Topographic Index Stats...

\&type

\&type

\&work \%workdir\%

$/ *$ Cleanup

\&if [exists \%workdir\%/\%pre\%ount\%_cti -grid] \&then

kill \%workdir\%/\%pre\%count\%_cti äll

sif [exists $\%$ workdir\%/\%pre\%count\% cti_p -cover] \&then

kill \%workdir\%/\%pre\%count\%_cti_p al]

/*Clip Elevation Data to Subbasin

latticeclip \%cti\% \%workdir\%/\%pre $\% \operatorname{count} \%$ workdir $\%$ \%re $\%$ count $\%$ cti

/**** Calculate Summary Statistics to Apply Back to Master Subbasin Coverage **** \&describe \%workdir\%/\%pre \% $\%$ count $\%$ cti

\&SV cti $\min =\% \mathrm{GRD}$ ZMINo

\&SV cti max $=\%$ GRD\$ZMAX

\&SV Cti_mean = \%GRD \$MEAN\%

$\&$ SV Cti Sd $=\%$ GRD STDV

*Additems to Basin Coverage

\&work \%vectordir\%

tables

sel $\%$ subbasin\%.pat

\&sv itemexist $=$ [iteminfo $\%$ subbasin .pat -info cti_min -exists] 
\&if $\%$ itemexist: $=$.FALSE. \&then

additem $\%$ subbasin\%.pat cti_min $88 \mathrm{~N} 3$

\&delvar itemexist

\&sv itemexist $=$ [iteminfo $\%$ subbasin\%.pat -info cti_max -exists]

\&if \%itemexist $=$.FALSE. \&then

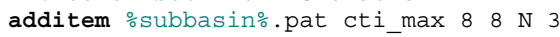

\&delvar itemexist

\&sv itemexist $=$ [iteminfo $\%$ subbasin\%.pat -info cti mean -exists]

\&if \%itemexist\% $=$.FALSE. \&then

additem $\%$ subbasin\%.pat cti_mean $88 \mathrm{~N} 3$

\&delvar itemexist

\&sv itemexist $=$ [iteminfo $\%$ subbasin .pat -info cti sd -exists]

\&if $\%$ itemexist $=$.FALSE. \&then

additem \%subbasin\%.pat cti_sd $88 \mathrm{~N} 3$

\&delvar itemexist

sel $\%$ subbasin .pat

resel $\%$ sb_item $\%$ count $\%$

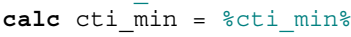

calc cti $\max =\frac{\circ}{c} \mathrm{cti}^{-} \max \%$

calc cti mean $=$ octi mean

calc cti sd $=\frac{\circ}{c}$ cti $\mathrm{s} \overline{\mathrm{d}} \%$

sel

quit /*Out of Tables

/*Remove Temporary Data

kill \%workdir\%/\%pre\% count\% cti all

/*kill \%workdir\%/\%pre \% count\% cti p all

\&work $\%$ scriptdir\%

\&return

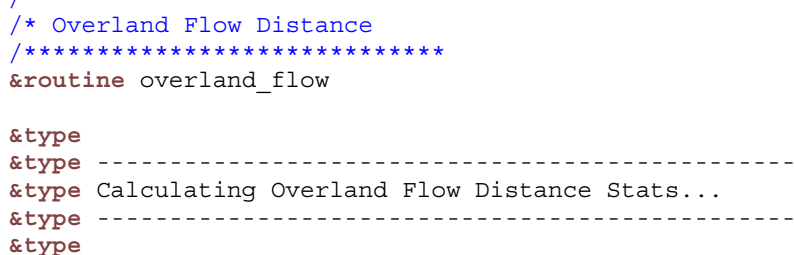

\&work \%workdir\%

/ Cleanup

\&if [exists \%pre\%count\% olf -grid] \&then

kill \%pre\%count\%_olf àll

/*Clip Elevation Data to Subbasin

latticeclip $\%$ olfd\% \%pre\%count\% \%pre\%count\%_olf

\&describe $\%$ pre $\%$ count $\%$ olf

\&SV Olfd_min $=$ \%GRD\$ZMIN\%

$\&$ SV Olfd_max $=\circ$ GRD $\$$ ZMAX\%

\&SV olfd mean = $\%$ GRD $\$ M E A N \circ$

$\&$ Sv olfd_sd $=\circ$ GRD $\$ S T D V \%$

/*Remove Data

kill \%pre\%count\%_olf all

/*Additems to Basin Coverage \&work \%vectordir\%

tables

\&sv itemexist $=[$ iteminfo $\%$ subbasin\%.pat - info olfd_min -exists $]$

\&if $\%$ itemexist $\%$.FALSE. \&then

additem $\%$ subbasin\%.pat olfd min $88 \mathrm{~N} 3$

\&delvar itemexist

$\&$ sv itemexist $=[$ iteminfo $\%$ subbasin\%.pat -info olfd_max -exists $]$

\&if \%itemexist $\%$.FALSE. \&then

additem $\%$ subbasino.pat olfd $\max 88 \mathrm{~N} 3$

\&delvar itemexist

\&sv itemexist $=[$ iteminfo $\%$ subbasin\%.pat -info olfd_mean - exists $]$ 
\&if \%itemexist\% = .FALSE. \&then

additem $\%$ subbasin\%.pat olfd_mean $88 \mathrm{~N} 3$

\&delvar itemexist

\&sv itemexist $=$ [iteminfo \%subbasin .pat -info olfd sd -exists $]$

\&if $\frac{\circ}{0}$ temexist $\%$. FALSE. \&then

additem \%subbasin\%.pat olfd_sd $8 \quad 8$ N 3

\&delvar itemexist

sel $\%$ subbasin\%.pat

sel $\%$ subbasin\%.pat

resel $\%$ sb_item\% $=\%$ count $\%$

calc olfd_min $=\%$ olfd min

calc olfd max $=\%$ olfd max

calc olfd mean $=$ \%olfd mean

calc olfd_sd $=\% \circ \mathrm{fd}$ s $\mathrm{s}$ \%

sel

quit /*out of Tables

/* Cleanup

\&work \%workdir\%

\&if [exists \%pre\% count\%_olf -cover] \&then

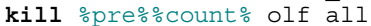

\&work $\%$ scriptdir\%

\&return

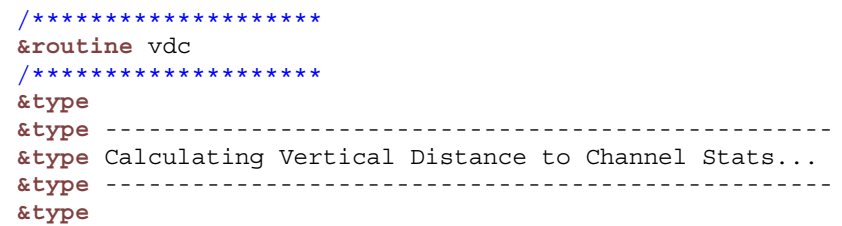

\&work \%workdir\%

$/ *$ Cleanup

\&if [exists \%workdir\%/\%pre\%count\% vdc -grid] \&then

kill \%workdir\%/\%pre\%count\% vdc all

\&if [exists $\%$ workdir\%/\%pre\% $\%$ count\%_vdc_p -cover] \&then

kill \%workdir\%/\%pre\%count\%_vdc_p $\bar{p}$ al $\bar{l}$

/*Clip Elevation Data to Subbasin

latticeclip \%vdc\% \%workdir\%/\%pre \% count \% \% workdir\%/\%pre \%

/**** Calculate Summary Statistics to Apply Back to Master Subbasin Coverage **** \&describe \%workdir\%/\%pre\% $\%$ count $\div$ vdc

\&SV vdC_min $=\circ$ GRD $\$$ ZMIN $\%$

\&SV vdC_max $=\%$ GRD $\$$ ZMAX

$\& \mathrm{SV}$ VdC mean = \%GRD $M E A N \circ$

$\& \mathrm{SV}$ VdC $\mathrm{sd}=\% \mathrm{GRD} \$ \mathrm{STDV}$

/*Additems to Basin Coverage \&work \%vectordir\%

tables

sel $\%$ subbasin\%.pat

\&sv itemexist $=[$ iteminfo $\%$ subbasino.pat - info vdc min -exists $]$

\&if $\%$ itemexist\% $=$.FALSE. \&then

additem \%subbasin:pat vdc_min $88 \mathrm{~N} 3$

\&delvar itemexist

\&sv itemexist $=[$ iteminfo $\%$ subbasino.pat - info vdc $\max$-exists $]$

\&if $\%$ itemexist: $=$.FALSE. \& then

additem \%subbasin\%.pat vdc_max $88 \mathrm{~N} 3$

\&delvar itemexist

\&sv itemexist $=$ [iteminfo $\%$ subbasino.pat -info vdc mean -exists]

\&if \%itemexist\% $=$.FALSE. \&then

additem $\%$ subbasin\%.pat vdc_mean $88 \mathrm{~N} 3$

\&delvar itemexist

\&sv itemexist $=$ [iteminfo $\%$ subbasin .pat - info vdc sd -exists $]$

\&if $\%$ itemexist $=$.FALSE. \&then

additem \%subbasin\%.pat vdc_sd $88 \mathrm{~N} 3$

\&delvar itemexist 
sel $\%$ subbasin\%.pat

resel $\%$ sb_item\% = $\%$ count $\%$

calc vdc $\min =\%$ vdc $\min \%$

calc vdc $\max =\frac{\circ}{\circ} \mathrm{vdc} \max \%$

calc vdc mean $=\frac{\circ}{0} \mathrm{vdc}$ mean

calc vdc_sd $=\%$ vdc_s $s \overline{\mathrm{d}}$

sel

quit /*Out of Tables

/*Remove Temporary Data

kill \%workdir\%/\%pre\% $\%$ count\% vdc all

/*kill \%workdir\%/\%pre\%count\%_vdc_p all

\&work 어iptdir\%

\&return

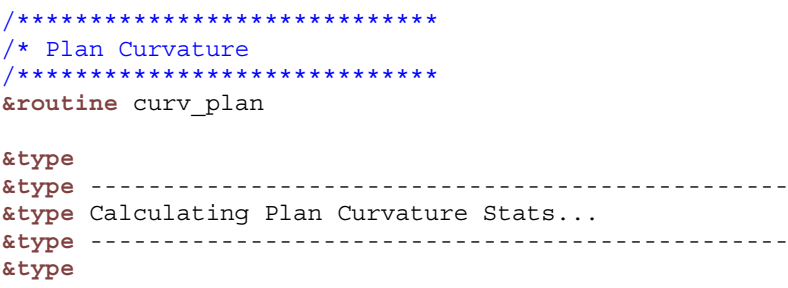

\&work \%workdir\%

/* Cleanup

\&if [exists $\%$ pre $\%$ count $\%$ pcv -grid] \& then

kill \%pre\%count\%_pcv all

/*Clip Elevation Data to Subbasin

latticeclip \%curv_plan\% \%pre\%

\&describe $\%$ pre $\%$ count $\%$ pcv

$\&$ SV $\mathrm{pCV} \min =\% \mathrm{GRD} \$ \mathrm{ZMIN}$

$\&$ SV PCV max $=\circ$ GRD $\$$ ZMAX

$\& \mathbf{S V}$ PCV mean = $\mathrm{GRD} \$ \mathrm{MEAN} \%$

$\&$ Sv pCV_sd $=\circ \mathrm{GRD} \$ \mathrm{STDV}$

/ Remove Data

kill $\%$ pre\%은

/*Additems to Basin Coverage

\&work \%vectordir\%

tables

\&sv itemexist $=$ [iteminfo $\%$ subbasin .pat -info plncrv min -exists]

\&if oitemexist: $=$.FALSE. \& then

additem $\%$ subbasin:pat plncrv_min $88 \mathrm{~N} 3$

\&delvar itemexist

\&sv itemexist $=$ [iteminfo \%subbasin\%.pat -info plncrv max -exists]

\&if $\%$ itemexist $=$. FALSE. \& then

additem \%subbasin\%.pat plncrv_max $88 \mathrm{~N} 3$

\&delvar itemexist

\&sv itemexist $=[$ iteminfo $\%$ subbasin\%.pat - info plncrv mean - exists $]$

\&if $\%$ itemexist $=$. FALSE. \&then

additem $\%$ subbasin\%.pat plncrv_mean $88 \mathrm{~N} 3$

\&delvar itemexist

$\&$ sv itemexist $=$ [iteminfo $\%$ subbasin\%.pat - info plncrv sd -exists $]$

\&if \%itemexist $=$. FALSE. \&then

additem $\%$ subbasin\%.pat plncrv_sd $88 \mathrm{~N} 3$

\&delvar itemexist

sel \%subbasin\%.pat

sel $\div$ subbasin\%.pat

resel $\%$ sb_item $\%$ count $\%$

calc plncrv min $=\frac{\circ}{p} \mathrm{cv}$ min

calc plncrv max $=\frac{\circ}{p c v} \max \%$

calc plncrv_mean $=\circ \mathrm{pc} \overline{\mathrm{v}}$ mean

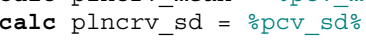




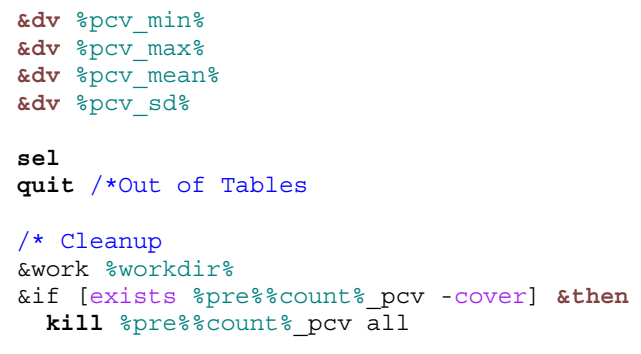

\&work $\%$ scriptdir\%

\&return

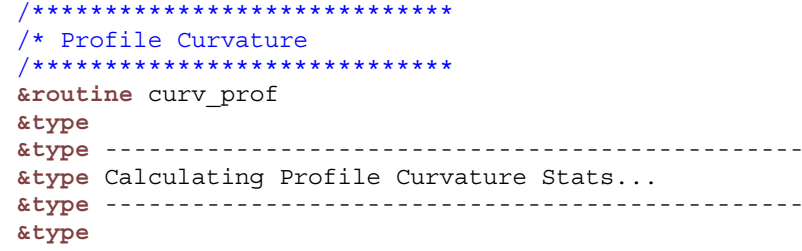

\&work \%workdir\%

/* Cleanup

\&if [exists \%pre\%count\%-pcv -grid] \&then kill $\%$ pre\% $\%$ count $\%$ pcv all

/*Clip Elevation Data to Subbasin

latticeclip \%curv_prof\% \%

\&describe $\%$ pre $\%$ count $\%$ pcv

\&SV pCV $\min =\frac{\circ}{\mathrm{GR} D} \$ \mathrm{ZMIN}$

\&SV PCV_max $=\%$ GRD $\$$ ZMAX\%

$\&$ Sv pcV_mean = \%GRD\$MEAN\%

$\&$ SV pCV_Sd $=\%$ GRD $\$ S T D V \%$

/*Remove Data

kill \%pre\%\%count\%_pcv all

*Additems to Basin Coverage

\&work \%vectordir\%

tables

\&sv itemexist $=$ [iteminfo \%subbasino.pat -info prfarv_min -exists] \&if \%itemexist\% $=$.FALSE. \&then

additem $\%$ subbasin $\%$ pat prfcrv_min $88 \mathrm{~N} 3$

\&delvar itemexist

\&sv itemexist $=$ [iteminfo \%subbasino.pat -info prfarv_max -exists]

\&if \%itemexist $=$.FALSE. \&then

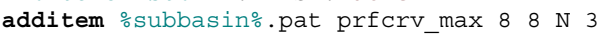

\&delvar itemexist

\&sv itemexist $=$ [iteminfo $\%$ subbasin\%.pat -info prfcrv_mean - exists]

\&if \%itemexisto = . FALSE. \&then

additem $\%$ subbasino.pat prfcrv mean $88 \mathrm{~N} 3$

\&delvar itemexist

\&sv itemexist $=$ [iteminfo \%subbasino.pat -info prfcrv_sd -exists]

\&if \%itemexist\% $=$. FALSE. \&then

additem $\%$ subbasin\%.pat prferv sd $88 \mathrm{~N} 3$

\&delvar itemexist

sel \%subbasin\%,pat

sel $\%$ subbasin\%.pat

resel $\%$ sb item $\%$ count $\%$

calc prferv_min $=\circ$ pcv_min $\%$

calc prfcrv_max $=\circ$ pcv_max

calc prfcrv_mean $=\frac{\circ}{\mathrm{p} c \bar{v}}$ mean

calc prferv_sd $=\%$ pcv_s $\overline{0} \%$

$\& d v \div p c v \_m i n \circ$

$\& d v \div p c v \_$max。

$\& d v$ opcv mean: 
$\& d v \div p c v \_s d \%$

sel

quit /*Out of Tables

* Cleanup

\&work 。workdir\%

sif [exists $\%$ pre\% count\%_pcv -cover] \&then

kill \%pre\%\%count\%_pcv all

\&work ㅇriptdir\%

\&return

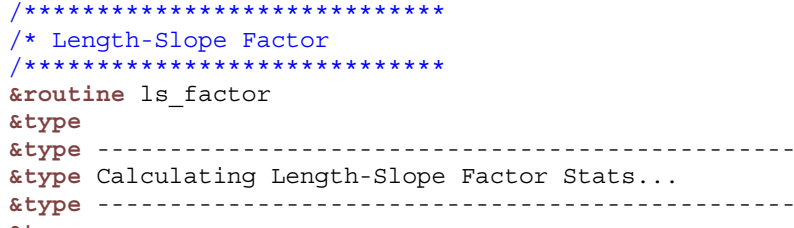

\&work workdir\%

/* Cleanup

\&if [exists $\%$ pre $\%$ count $\%$ lsf -grid] \&then

kill $\%$ pre\%ount\%_lsf all

/*Clip Elevation Data to Subbasin

latticeclip \%ls_factor\% \%pre\% $\%$ count $\%$ pre $\%$ count $\%$ lsf

\&describe $\%$ pre $\%$ count\%_lsf

\&SV Isf_min $=\circ \mathrm{GRD} \$ \mathrm{ZMIN}$

\&SV Isf_max $=\%$ GRD $\$$ ZMAX\%

$\&$ SV lsf mean $=\div$ GRD $\$ M E A N \div$

\&SV lsf_sd $=\% \mathrm{GRD} \$ \mathrm{STDV} \%$

/*Remove Data

kill \%pre\%ㅇount\%_lsf all

/*Additems to Basin Coverage

\&work \%vectordir\%

\section{tables}

\&sv itemexist $=$ [iteminfo $\%$ subbasin\%.pat -info lsfact_min -exists] \&if \%itemexist $\%$.FALSE. \&then

additem $\%$ subbasin\%.pat lsfact $\min 88 \mathrm{~N} 3$

\&delvar itemexist

\&sv itemexist $=$ [iteminfo $\%$ subbasin\%.pat -info lsfact_max -exists] \&if $\%$ itemexist $\%$.FALSE. \&then

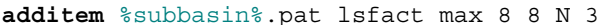

\&delvar itemexist

\&sv itemexist $=$ [iteminfo $\%$ subbasin\%.pat -info lsfact_mean - exists] \&if $\%$ itemexisto $=$. FALSE. \&then

additem $\%$ subbasin\%.pat lsfact mean $88 \mathrm{~N} 3$

\&delvar itemexist

\&sv itemexist $=$ [iteminfo $\%$ subbasin\%.pat -info lsfact_sd - exists $]$

\&if $\%$ itemexist $\%$. FALSE. \&then

additem $\%$ subbasin\%.pat lsfact sd $88 \mathrm{~N}$

\&delvar itemexist

sel $\%$ subbasin\%.pat

sel \%subbasin pat

resel $\%$ sb item $\%=$ count $\%$

calc lsfact_min $=\%$ lsf min

calc lsfact_max $=\%$ Isf_max

calc lsfact mean $=\%$ lsf mean

calc lsfact $\mathrm{sd}=\%$ lsf $\mathrm{sd} \%$

sel

quit /*Out of Tables

/* Cleanup

swork \%workdir:

\&if [exists \%pre\%count\%lsf -cover] \&then

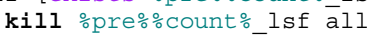


\&work ㅇciptdir\%

\&return

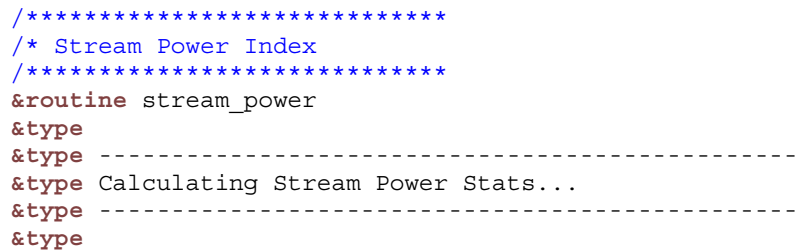

\&work \%workdir\%

/* Cleanup

\&if [exists $\%$ pre $\%$ count $\%$ lsf -grid] \&then

kill \%pre\%count\%_lsf all

/*Clip Elevation Data to Subbasin

latticeclip \%strmpower\% \%pre\% count\% \%pre\%count $\%$ pwr

\&describe $\%$ pre $\%$ count $\%$ pwr

\&SV PwI_min $=\div$ GRD\$ZMIN\%

$\&$ SV PWr max $=\div$ GRD $\$ Z M A X \circ$

$\&$ SV pwr mean = $\%$ GRD $\$ M E A N \circ$

\&SV PWI Sd $=\circ$ GRD $\$ S T D V$

/*Remove Data

kill \%pre\%ㅇount\%_pwr all

/*Additems to Basin Coverage \&work vectordir\%

tables

\&sv itemexist $=$ [iteminfo $\%$ subbasin\%.pat -info strpwr_min -exists] \&if $\%$ itemexist $\%$.FALSE. \&then

additem \%subbasin\%.pat strpwr min $88 \mathrm{~N} 3$ \&delvar itemexist

\&sv itemexist $=$ [iteminfo $\%$ subbasin\%.pat -info strpwr_max -exists] \&if \%itemexist $=$.FALSE. \&then

additem $\%$ subbasin\%.pat strpwr $\max 88 \mathrm{~N} 3$ \&delvar itemexist

\&sv itemexist $=$ [iteminfo \%subbasin\%.pat -info strpwr_mean -exists $]$ \&if $\%$ itemexist $\%$.FALSE. \&then

additem $\%$ subbasin\%.pat strpwr mean $88 \mathrm{~N} 3$ \&delvar itemexist

\&sv itemexist $=$ [iteminfo $\%$ subbasin\%.pat -info strpwr_sd -exists]

\&if \%itemexist $=$.FALSE. \&then

additem $\frac{\circ}{\text { subbasin }}$.pat strpwr sd $88 \mathrm{~N} 3$

\&delvar itemexist

sel $\%$ subbasin\%.pat

sel \%subbasin\%.pat

resel $\div$ sb_item\% $=\frac{\circ}{\circ}$ count $\%$

calc strpwr_min $=\frac{\circ}{\mathrm{p} w} \mathrm{w}_{-} \min \%$

calc strpwr max $=\frac{\circ}{0}$ w max

calc strpwr mean $=\frac{\circ}{0}$ pr mean

calc strpwr $\mathrm{sd}=\frac{\circ}{0} \mathrm{pwr}$ s $\overline{\mathrm{d}} \%$

quit /*Out of Tables

/* Cleanup

\&work \%workdir:

\&if [exists $\%$ pre $\%$ count $\%$ pwr -cover] \&then

kill $\frac{\circ}{0}$ pro $\%$ count $\%$ pwr all

\&work 으iptdir\%

\&return 


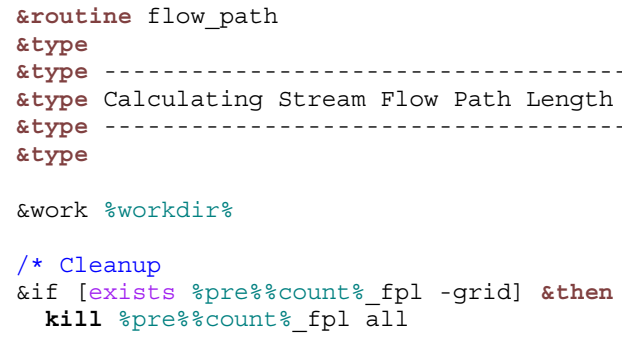

/*Clip Elevation Data to Subbasin

latticeclip $\%$ fwpath\% \%pre\%count \% \%

\&describe \%pre\% $\%$ count $\%$ pwr

\&SV fpl min $=\%$ GRD $\$$ ZMIN

\&SV fpl max $=\circ$ GRD $\$ Z M A X \%$

\&SV fpl mean = \%GRD\$MEAN응

$\& S V$ fpl_sd =

/*Remove Data

kill \%pre\%은 fpl all

/*Additems to Basin Coverage \&work \%vectordir\%

tables

\&sv itemexist $=$ [iteminfo $\%$ subbasin .pat -info flwlng_min -exists]

\&if $\%$ itemexist $\%$.FALSE. \&then

additem $\%$ subbasino.pat flwlng min $88 \mathrm{~N} 3$

\&delvar itemexist

\&sv itemexist $=[$ iteminfo $\%$ subbasino.pat -info flwlng_max -exists $]$

\&if $\frac{\circ}{0}$ temexist $\%$. FALSE. \&then

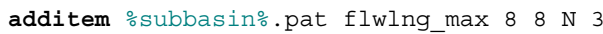

\&delvar itemexist

\&sv itemexist $=$ [iteminfo $\%$ subbasin. pat - info flwlng mean -exists $]$

\&if $\%$ itemexist $=$.FALSE. \&then

additem $\%$ subbasin\%.pat flwlng_mean $88 \mathrm{~N} 3$

\&delvar itemexist

\&sv itemexist $=$ [iteminfo $\%$ subbasino.pat - info flwlng sd -exists]

\&if \%itemexist\% $=$. FALSE. \&then

additem $\%$ subbasin\%.pat flwlng_sd $88 \mathrm{~N} 3$

\&delvar itemexist

sel \%subbasin\%.pat

sel $\%$ subbasin .pat

resel $\%$ sb_item $\%$ count $\%$

calc flwlng $\min =\circ \mathrm{fpl} \min \%$

calc flwlng max $=\% \mathrm{fpl}$ max。

calc flwling mean $=\% \mathrm{fp} \bar{l}$ mean

calc flwlng_sd $=\circ \mathrm{fpl}$ s $\mathrm{s} \overline{\mathrm{d}} \%$

sel

quit /*out of Tables

/* Cleanup

\&work \%workdir\%

\&if [exists \%pre\% $\%$ count $\%$ fpl -cover] \&then

kill \%pre\%count\% fpl all

\&work $\div$ scriptdir\%

\&return

$/ * * * * * * * * * * * * * * * * * * * *$

\&routine tci

t*********************

\&type

- - - - - -

\&type Calculating Topographic Convergence Index Stats...

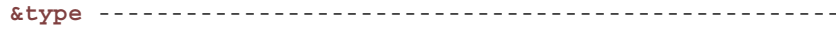

\&type

\&work \%workdir\% 


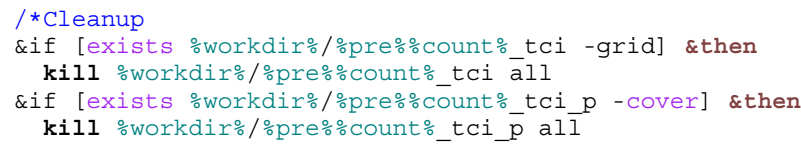

/*Clip Elevation Data to Subbasin

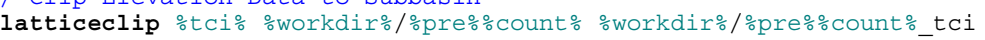

/**** Calculate Summary Statistics to Apply Back to Master Subbasin Coverage **** \&describe \%workdir\%/\%pre\%count\%_tci

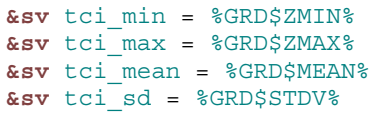

\section{tables}

sel $\%$ subbasin .pat

\&sv itemexist $=[$ iteminfo $\%$ subbasin\%.pat - info tci_min -exists $]$

\&if \%itemexist $=$.FALSE. \&then

additem $\%$ subbasin\%.pat tci_min $88 \mathrm{~N} 3$

\&delvar itemexist

\&sv itemexist $=$ [iteminfo $\%$ subbasin\%.pat - info tci_max -exists]

\&if \%itemexist\% $=$. FALSE. \&then

additem $\%$ subbasin\%.pat tci $\max 88 \mathrm{~N} 3$

\&delvar itemexist

\&sv itemexist $=$ [iteminfo \%subbasino.pat -info tci_mean - exists]

\&if $\%$ itemexist $=$.FALSE. \&then

additem $\%$ subbasin\%.pat tci mean $88 \mathrm{~N} 3$

\&delvar itemexist

\&sv itemexist $=$ [iteminfo \%subbasin\%.pat -info tci_sd -exists]

\&if $\%$ itemexist $\%$. FALSE. \&then

additem \%subbasin\%.pat tci sd $88 \mathrm{~N} 3$

\&delvar itemexist

sel $\%$ subbasin\%.pat

resel $\%$ sb item $\%$ count $\%$

calc tci $\min =\%$ tci $\min \%$

calc tci_max $=\%$ tci max\%

calc tci_mean = \%tci mean

calc tci_sd = \%tci_s $\mathrm{d}$ \%

sel

quit /*Out of Tables

/*Remove Temporary Data

kill \%workdir\%/\%pre\%

/*kill \%workdir\%/\%pre\% \%ount\%_tci_p all

\&work $\frac{\circ}{\text { scriptdir\% }}$

\&return

\&routine topofeature

$1 * * * * * * * * * * * * * * * * * * * *$

\&type

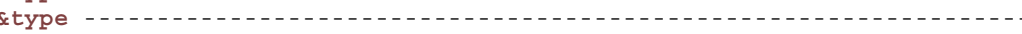

\&type Determining Percent Area of each Topographic Feature Class..

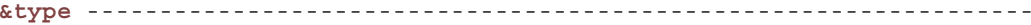

\&type

\&work \%workdir\%

/* Cleanup

\&if [exists $\%$ pre $\%$ count\% tpf -grid] \& then

kill \%pre\%count\%_tpf àll

*Clip Topographic Feature GRID to Individual Subbasin

latticeclip \%topof\% \%pre $\%$ count $\%$ opre $\%$ count $\%$ tpf

\&describe $\%$ pre\% $\%$ count $\%$ tpf 


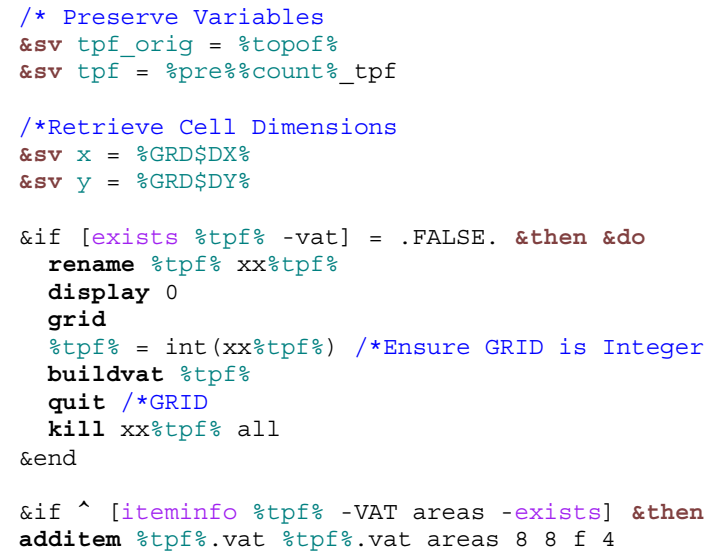




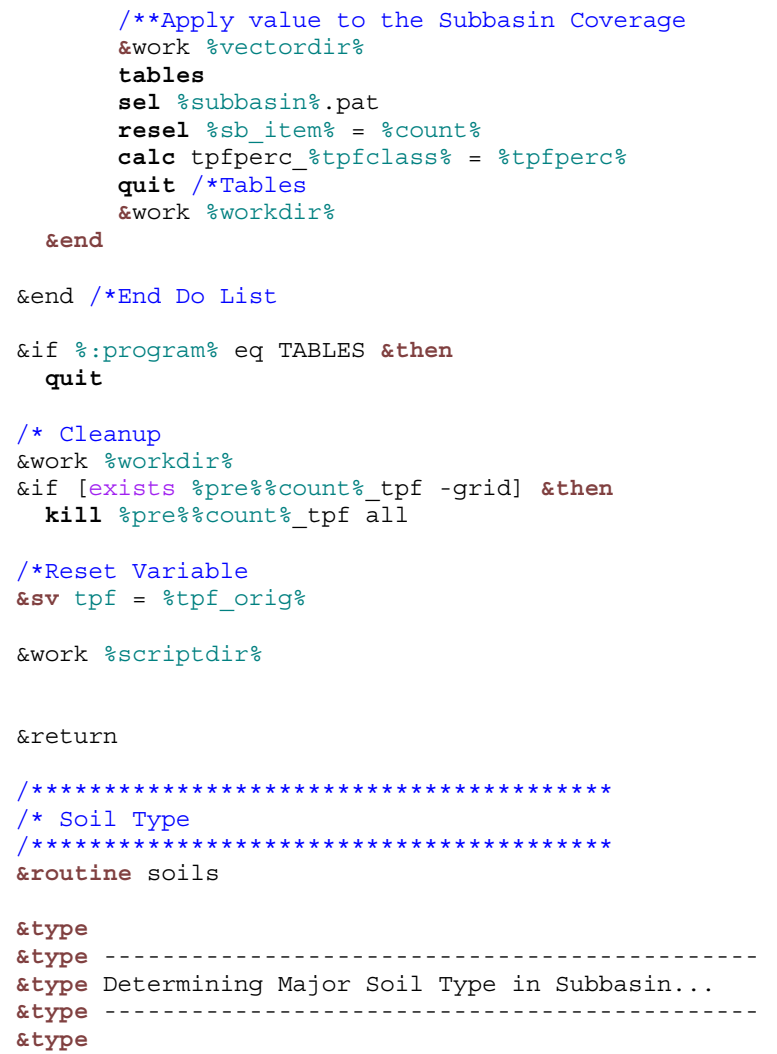

/*Clip Soils Data to Subbasin

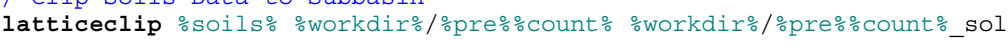

/*Determine Number of Classes in Clipped Soils GRID

\&describe \%workdir\%/\%pre \% $\%$ count $\%$ sol

\&SV soil_units $=\circ$ GRD $\$ N C L A S S \circ$

$/ *$ Change to Work Directory

\&work oworkdir:

/*Fire up tables if need be

\&if : program\% ne TABLES \&then

tables

/*Pull Values from VAT

sel \%pre\%\%ount:_sol.vat

\&sv gcount $=1$

$\&$ sv $\operatorname{vall}=0$

\&sv cellnum $=0$

\&do \&until \%gcount: gt \%soil_units:

$\&$ SV val $=[$ SHOW RECORD \%gCount: ITEM count $]$

\&SV vall = \%Val1\% \%Val\%

\&sv cellnum $=\left[\right.$ calc $\%$ cellnum $\left.+\frac{\circ}{\circ} v a l \%\right] / *$ Sum the total number of cells

$\&$ Sv gcount $=[\mathrm{calc} \div \mathrm{c}$ gcount $\%$ 1]

\&end

\&sv vall $=$ \%vall\%

\&SV domsoil $=[$ extract 1 [sort \%Val1\% -DESCEND -NUMERIC]

resel count $=$ \%domsoil $\%$

/*Calc Percent Area of Dominant Soil

\&sv dsol area $=[\mathrm{calc}[\mathrm{calc} \%$ domsoil\% / \%cellnum\% $]$ * 100]

\&if [exists domsoil.tmp -info] \&then

kill domsoil.tmp

copy \%pre\% $\%$ count $\%$ sol.vat domsoil.tmp DATA

sel domsoil.tmp

\&sv domsoil_val1 = [SHOW RECORD 1 ITEM value]

sel

kill domsoil.tmp

\&dv val

quit /*Out of Tables

/*Write Variables to Sub-Basin Coverage 
\&work \%vectordir\%

/*Additems to SubBasin Coverage tables

sel \%subbasin\%.pat

\&sv itemexist $=$ [iteminfo \%subbasin\%.pat -info dsoil_type -exists]

\&if \%itemexist $=$. FALSE. \&then

additem $\%$ subbasin. pat dsoil type 33 I

\&delvar itemexist

\&sv itemexist $=$ [iteminfo \%subbasin\%.pat -info dsoil_perc-exists]

\&if $\%$ itemexist $=$.FALSE. \&then

additem \%subbasin\%.pat dsoil perc $66 \mathrm{~N} 3$

\&delvar itemexist

sel \%subbasin\%.pat

calc dsoil type = \%domsoil vall\%

calc dsoil perc $=\circ d$ sol area

sel

quit /*Out of Tables

/*Cleanup

kill \%workdir\%/\%pre\% Count\%_sol all

\&work \%scriptdir\%

\&return

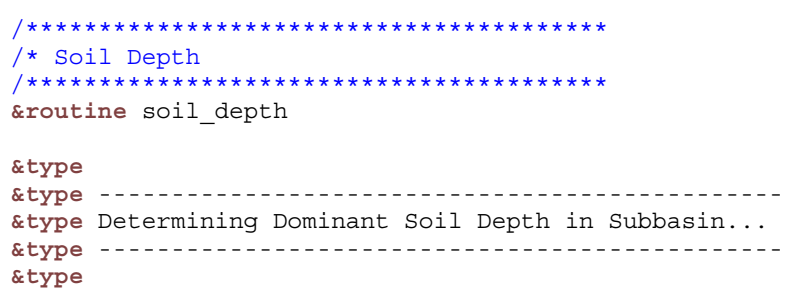

/*Clip Soil Depth Data to Subbasin

latticeclip $\%$ soil_depth\% \%workdir\%/\%pre $\%$ count $\%$ workdir\%/\%pre\%

/*Determine Number of Classes in Clipped Soils GRID

\&describe \%workdir\%/\%pre\%count\%_sld

$\&$ Sv soil units $=\circ$ GRD \$NCLASS

/*Change to Work Directory

\&work \%workdir\%

*Fire up tables if need be

\&if : program\% ne TABLES \&then

tables

/*Pull Values from VAT

sel $\%$ pre $\%$ count $\%$ sld.vat

\&sv gcount $=1$

\&sv vall $=0$

\&do suntil gcount\% gt \%soil_units\%

\&Sv val = [SHOW RECORD \%gcount\% ITEM count]

\&sv vall = \%vall\% \%val\%

$\&$ Sv gcount $=[\mathrm{calc} \% \mathrm{gcount} \%+1]$

\&end

\&sv vall = \%val1\%

\&Sv domsoild $=$ [extract 1 [sort \%val1\% -DESCEND -NUMERIC] ]

resel count $=\%$ domsoild\%

\&if [exists domsoild.tmp -info] \&then

kill domsoild.tmp

copy \%pre\%count $\%$ sld.vat domsoild.tmp DATA

sel domsoild.tmp

\&sv domsoild val1 = [SHOW RECORD 1 ITEM value $]$

sel

kill domsoild.tmp

$\& d v$ val

quit /*Out of Tables

/*Write Variables to Sub-Basin Coverage \&work \%vectordir\%

*Additems to SubBasin Coverage tables 


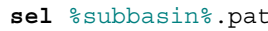

\&Sv itemexist $=$ [iteminfo \%subbasino.pat -info soil_depth - exists]

\&if $\%$ itemexist $=$.FALSE. \&then

additem \%subbasin\%.pat soil_depth $66 \mathrm{~N} 3$

\&delvar itemexist

sel $\%$ subbasin\%pat

calc soil_depth = \%domsoild_val1\%

sel

quit /*Out of Tables

/*Cleanup

kill \%workdir\%/\%pre\% count\%_sld all

\&work \%scriptdir\%

\&return

$* * * * * * * * * * * * * * * *$

/* Curvature Classification

$/ * * * * * * * * * * * * * * * * * * * * * * * * * * *$

\&routine curv_class

\&type

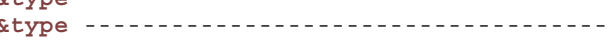

\&type Calculating Basin Sinuosity Stats...

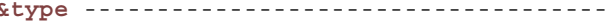

stype

$/ * * * * * * * * * * * * * *$ May Need to Do this as percent area $* * * * * * * * * * * * * *$

\&work \%workdir\%

/* Cleanup

\&if [exists $\frac{\circ}{\circ}$ re\%count $\%$ sin -grid] \&then

kill \%pre\%count\%_sin āll

/*Clip Sinuosity Data to Subbasin

latticeclip $\%$ sinuosity\% \%

\&describe $\%$ pre $\%$ count $\%$ sin

\&SV $\operatorname{sinu} \min =\%$ GRD $\$ Z M I N \%$

\&SV sinu $\max =\circ \mathrm{GRD} \$ \mathrm{ZMAX} \%$

$\&$ SV sinu_mean $=$ \%GRD $\$ M E A N$

\&SV sinu_sd $=\% \mathrm{GRD} \$ \mathrm{STDV} \%$

* Remove Data

kill $\%$ pre\%\%count\%_sin all

*Additems to Basin Coverage

\&work \%vectordir\%

tables

\&sv itemexist $=$ [iteminfo $\%$ subbasin\%.pat -info sinu min -exists]

\&if \%itemexist $=$. FALSE. \&then

additem \%subbasin:pat sinu_min $88 \mathrm{~N} 3$

\&delvar itemexist

\&sv itemexist $=$ [iteminfo $\%$ subbasin\%.pat -info sinu max -exists]

\&if \%itemexist $=$. FALSE. \&then

additem $\%$ subbasino.pat sinu_max $88 \mathrm{~N} 3$

\&delvar itemexist

\&sv itemexist $=$ [iteminfo $\%$ subbasin\%.pat - info sinu mean - exists]

\&if \%itemexist $=$. FALSE. \&then

additem $\%$ subbasin\%. pat sinu_mean $88 \mathrm{~N} 3$

\&delvar itemexist

\&sv itemexist $=$ [iteminfo $\%$ subbasin\%.pat - info sinu sd - exists $]$

\&if \%itemexist\% $=$. FALSE. \&then

additem \%subbasin\%.pat sinu_sd $88 \mathrm{~N} 3$

\&delvar itemexist

sel \%subbasin\%.pat

resel $\%$ sb_item $\%$ count $\%$

calc $\operatorname{sinu} \min =\% \operatorname{sinu}$ min

calc sinu $\max =\frac{\circ}{\operatorname{sinu}} \max \%$

calc sinu mean $=\frac{\circ}{\circ} \sin \overline{\text { mean}} \%$

calc sinu_sd $=\%$ sinu_sdo 
sel

quit /*out of Tables

$/ *^{*}$ Cleanup

kill \%workdir\%/\%pre\%count $\%$ sin all

\&work $\%$ scriptdir\%

\&return

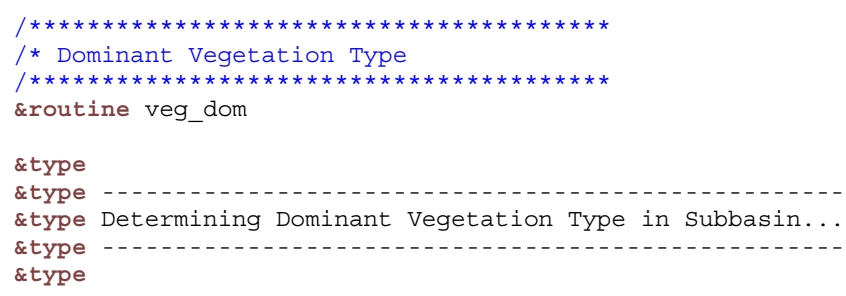

$/ *$ Clip Veg Data to Subbasin

latticeclip $\%$ veg\% $\%$ workdir\%/\%pre $\%$ Count $\%$ कWorkdir\%/\%pre\% $\%$ count $\%$ veg

/*Determine Number of Classes in Clipped Soils GRID \&describe $\%$ workdir\%/\%pre \% count $\%$ veg \&Sv veg units $=\circ$ GRD\$NCLASS응

\section{* Change to Work Directory} \&work oworkdir:

*Fire up tables if need be

\&if \%:program\% ne TABLES \&then tables

/*Pull Values from VAT

sel $\%$ pre $\%$ count $\%$ veg.vat

\&sv gcount $=1$

$\&$ sv $\operatorname{vall}=0$

\&sv cellnum $=0$

\&do \&until $\% g c o u n t \%$ gt \%veg units

\&Sv val $=[$ SHOW RECORD \%gcount $\%$ ITEM count $]$

$\&$ Sv vall = $\frac{\circ}{\circ}$ Val $1 \% \circ \mathrm{V} a 1 \%$

\&sv cellnum $=\left[\right.$ calc $\%$ cellnum $+\frac{\circ}{\circ}$ val\%] $/ *$ Sum the total number of cells \&sv gcount $=[$ calc $\%$ gcount $\%+1]$

\&end

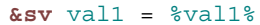

\&sv domveg $=[$ extract 1 [sort \%Val1\% -DESCEND -NUMERIC] ]

resel count $=$ \%domveg\%

*Calc Percent Area of Dominant Vegetation

\&sv dveg area $=[\mathrm{calc}[\mathrm{calc} \%$ domveg\% $/$ \%cellnum\%] * 100]

\&if [exists domveg.tmp -info] \&then

kill domveg.tmp

copy $\%$ pre $\%$ count $\%$ veg.vat domveg.tmp DATA

sel domveg.tmp

\&sv domveg_val1 = [SHOW RECORD 1 ITEM value]

sel

kill domveg.tmp

\&dv val

quit /*Tables

/*Write Variables to Sub-Basin Coverage \&work \%vectordir\%

/*Additems to SubBasin Coverage

\section{tables}

sel \%subbasin\%.pat

sv itemexist $=[$ iteminfo $\%$ subbasin .pat - info domveg type - exists $]$

\&if \%itemexist: $=$.FALSE. \& then

additem \%subbasin\%.pat domveg_type 33 I

\&delvar itemexist

\&sv itemexist $=[$ iteminfo $\%$ subbasin. pat - info domveg perc - exists]

\&if $\%$ itemexist $\%$. FALSE. \& then

additem \%subbasin\%.pat domveg_perc $66 \mathrm{~N} 2$

\&delvar itemexist

sel $\div$ subbasin .pat

calc domveg_type $=$ :domveg_vall:

calc domveg perc $=\circ d v e g$ area 
sel

quit /*out of Tables

$/ *$ Cleanup

kill \%workdir\%/\%pre\% \%count

\&work $\%$ scriptdir\%

\&return

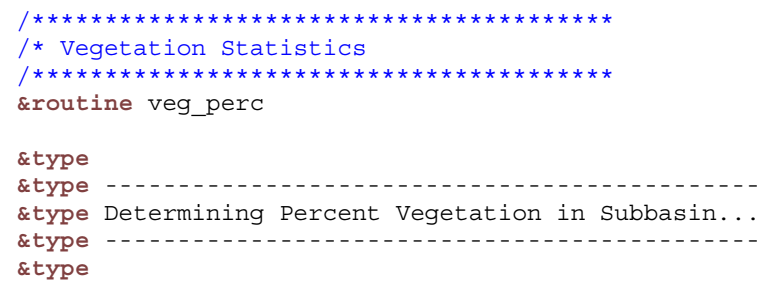

\&work \%workdir\%

/* Cleanup

\&if [exists $\%$ pre $\%$ count $\%$ veg -grid] \&then

kill $\frac{0}{p}$ re\%.count:_veg àll

/*Clip Vegetation Raster to Individual Subbasin

latticeclip $\%$ veg\% $\%$ pre $\%$ count $\%$ pre $\%$ count $\%$ veg

\&describe $\%$ pre\% $\%$ count $\%$ veg

\&sv veg_orig $=\frac{\circ}{\circ}$ veg\%

\&sv veg $=\frac{\circ}{\circ}$ pre $\%$ count $\%$ veg

/*Retrieve Cell Dimensions

$\&$ SV $\mathrm{X}=$ \% GRD $\$ D X \%$

$\&$ SV $Y=\circ G R D D Y \circ$

\&if [exists $\frac{\circ \text { veg\% }}{\text {-vat }]}=$.FALSE. \&then \&do

display 0

grid

buildvat veg\%

quit $/ *$ GRID

send

\&if $\wedge$ [iteminfo \%veg\% -VAT areas -exists] \&then additem $\%$ veg\%.vat $\%$ veg\%.vat areas $1414 \mathrm{n} 3$

\&if [iteminfo $\%$ Veg\% -VAT percent -exists] \&then additem $\%$ veg\%.vat $\%$ veg\%.vat percent 8 \& f 4

\&if ^ [iteminfo \%Veg\% -VAT sum -exists] \&then additem $\%$ veg\%. vat $\%$ veg\%. vat sum 88 f 4

/* Find Area of Subbasin

tables

sel $\%$ pre $\%$ count $\%$ veg.vat

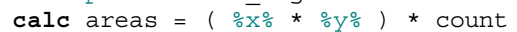

asel

statistics \# vegsum.dat

sum areas

end

\&sv basinarea $=$ [listunique vegsum.dat - info sum-areas]

kill vegsum.dat

/* Calc Percentage

calc percent $=($ areas $/$ obasinarea $) * 100$

quit /*Tables

\&work vectordir\%

/* Additems of all 12 Veg-Classes to Subbasin Coverage

\&do vegclass \&list $1 \begin{array}{llllllllllll}2 & 3 & 4 & 6 & 7 & 9 & 10 & 11 & 12\end{array}$

\&sv itemexist $=\left[\right.$ iteminfo $\%$ subbasin\% pat - info vegperc $-\frac{\circ}{\circ}$ vegclass $\%$-exists $]$

\&if $\%$ itemexist: $=$.FALSE. \&then

additem ․subbasin\%.pat \%subbasin\%. pat vegperc_ovegclass\% $66 \mathrm{n} 2$

\&delvar itemexist

\&end

\&work ㄴorkdir\% 


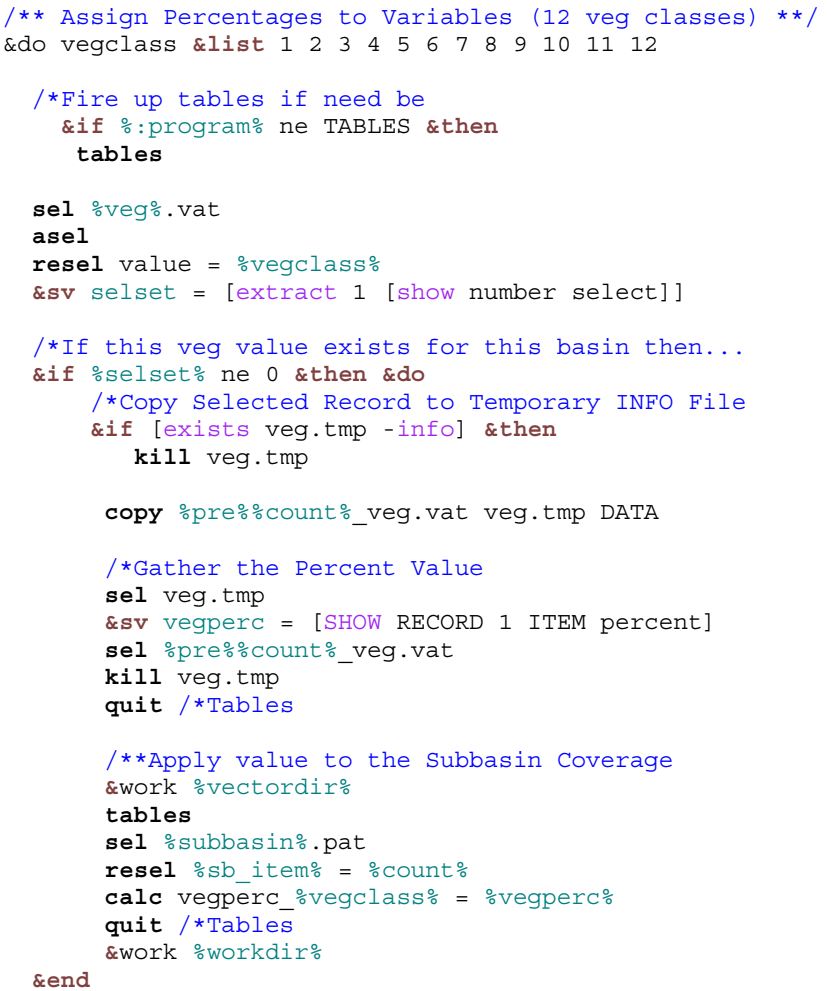

\&end /*End Do List

/*Quit tables if need be

\&if : program\% eq TABLES \&then quit

* Cleanup

\&work \%workdir\%

kill \%pre\% count\%_veg all

/* Reset Variable

\&sv veg = \%veg_orig\%

\&work \%scriptdir\%

\&return

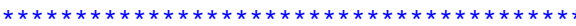

/* Topographic Roughness Index

/****************************************

\&routine tri

\&type

type Determining Percent Area of Each Topographic Roughness Class...

\&type

\&type

\&work workdir\%

/* Cleanup

\&if [exists $\%$ pre\% count $\%$ tri -grid] \&then

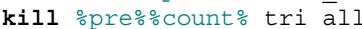

/*Clip Topographic Roughness GRID to Individual Subbasin

latticeclip \%tri\% \% pre\%count\% \%pre\%count\%_tri

\&sv tri orig $=\%$ tri\%

\&sv tri $=\frac{\circ}{\circ}$ pre $\%$ count $\%$ tri

/*Retrieve Cell Dimensions

\&describe \%pre $\%$ count $\%$ tri

\&SV $\mathrm{X}=\% \mathrm{GRD} \$ \mathrm{DX}$

$\& S V Y=\% G R D \$ D Y \circ$

\&if [exists $\%$ tri\%-vat] $=$.FALSE. \&then \&do

rename $\%$ tri\% $\mathrm{xx} \% \operatorname{tri} \%$ 


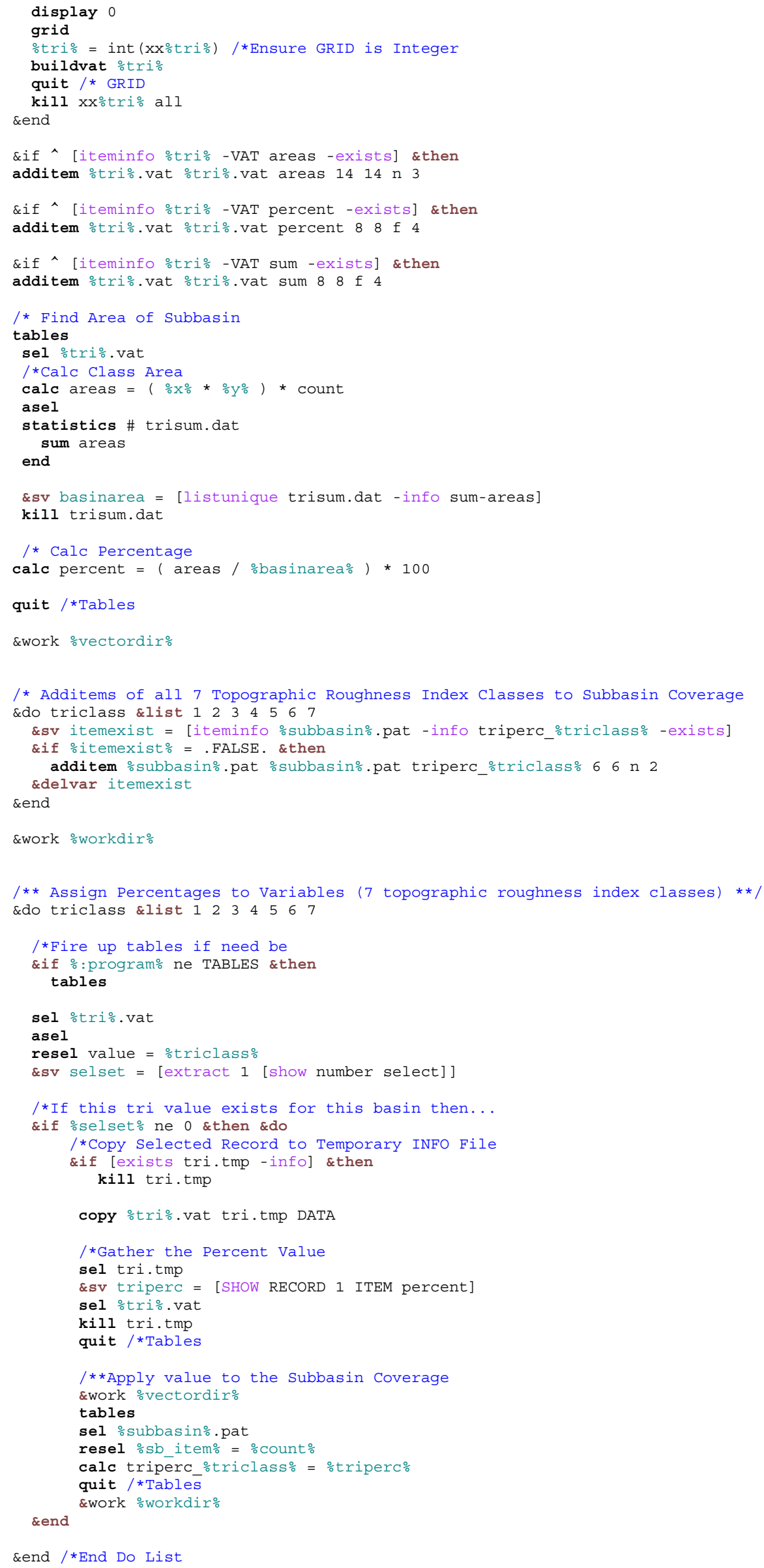




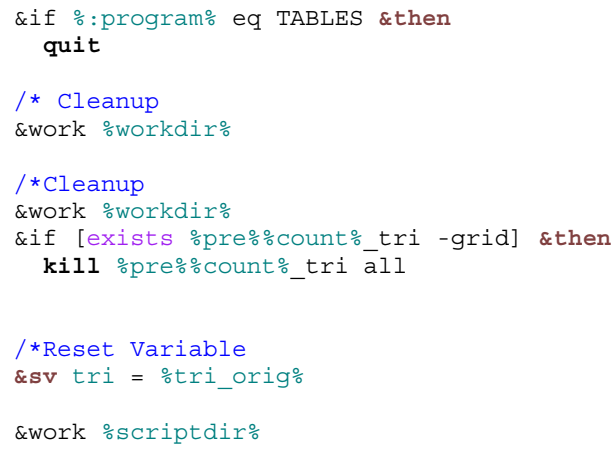

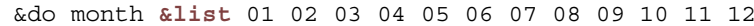

\&do type salist ppt tmin tmax 


\section{/*Additems to Basin Coverage} \&work \%vectordir\%

\section{tables}

\&sv itemexist $=$ [iteminfo $\%$ subbasin $\cdot$ pat -info slr_omonth\% mean - exists $]$ \&if \%itemexist: $=$.FALSE. \&then

additem $\%$ subbasin: pat slr_omonth\%_mean $88 \mathrm{~N} 3$

\&delvar itemexist

\&sv itemexist $=$ [iteminfo $\%$ subbasin .pat -info slr_\%month\%_sd - exists]

\&if \%itemexisto $=$.FALSE. \&then

additem $\%$ subbasin\%.pat slr_omonth\%_sd $88 \mathrm{~N} 3$

\&delvar itemexist

sel $\%$ subbasin\%.pat

sel $\%$ subbasin\%.pat

resel $\%$ sb item $\%$ = count

calc slr $\frac{\circ}{0}$ month\% mean $=\%$ slr mean\%

calc slr_omonth\%_sd = $\%$ slr_s $\overline{0} \%$

sel

quit /*Out of Tables

/ * Cleanup

\&work \%workdir\%

\&if [exists \%pre\% count\%_slr_omonth\%-cover] \&then

kill \%pre\% count\%_slr_\%month\% all

\&end / End Month Loop

\&work \%scriptdir\%

\&return

/*** Determine Sub-Basin Solar Insolation

\&routine insolation

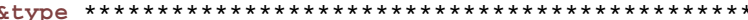

\&type Calculating Sub-Basin Solar Insolation Stats. .

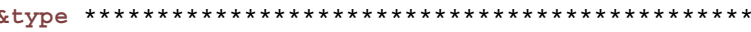

\&work \%workdir\%

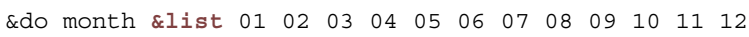

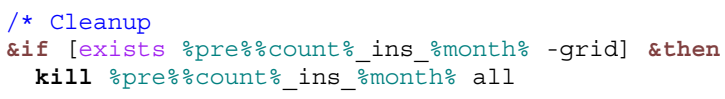

/*Clip Solar Data to Subbasin

latticeclip $\%$ insolation $\%$ month\% $\%$ pre $\%$ count $\%$ pre $\%$ count $\%$ ins_\%month

\&describe $\%$ pre \% count\%_ins_\%month\%

\&sv ins_mean $=\%$ GRD $\$ M E A N \%$

\&SV ins_sd $=\%$ GRD $\$$ STDV $\%$

/*Remove Data

kill \%pre\%\%count\%_ins_omonth\% all

/*Additems to Basin Coverage \&work ovectordir\%

\section{tables}

\&sv itemexist $=$ [iteminfo $\%$ subbasin\%.pat -info ins_omonth\% mean -exists] \&if \%itemexist: $=$. FALSE. \& then

additem $\%$ subbasin:pat ins_omonth\% mean $88 \mathrm{~N} 3$

\&delvar itemexist

\&sv itemexist $=$ [iteminfo $\%$ subbasin $\%$ pat - info ins_omonth\% ${ }_{-}$sd - exists $]$

\&if $\%$ itemexist\% $=$.FALSE. \&then

additem $\%$ subbasin\%.pat ins_omonth\%_sd $88 \mathrm{~N} 3$

\&delvar itemexist

sel \%subbasin\% pat

sel \%subbasin\%.pat 
resel $\%$ sb item $\%$ ㄷunt $\%$

calc ins $\%$ month\% mean $=\%$ ins mean

calc ins_omonth\%_sd = \%ins_s $\bar{d} \%$

sel

quit /*Out of Tables

/* Cleanup

\&work \%workdir\%

\&if [exist $s \%$ pre $\%$ count $\%$ ins $\%$ month $\%$-cover] \& then

kill \%pre\%count\% ins \%month\% all

send / End Month Loop

\&work \%scriptdir\%

\&return

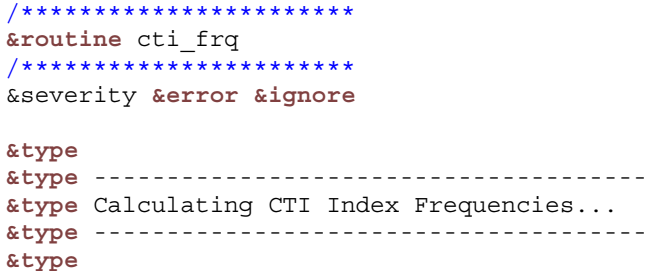

\&work ㅁose음

/*Determine The Percentile Interval (e.g. 20 will be every 5\% - 100/20 = 5) \&sv percentile $=20$

/*Convert GRID to Point Coverage \&if [exists cti_pt -cover] \&then kill cti pt all

\&severity \&warning \&ignore

gridpoint ti cti_pt value

\&severity \&warning \&fail

\&severity \&error \&fail

tables

additem cti_pt.pat code $44 \mathrm{~N} 1$

sel cti_pt.pat

calc code = value

quit

/***Calculate Frequency

/*Check if INFO File Exists

\&if [exists cti_pt.frq -info] \&then

killinfo cti pt.frq

frequency cti_pt.pat cti_pt.frq

code

end

end

/*Additem to Flag Values to be Dumped

additem cti_pt.frq cti_pt.frq extract $33 \mathrm{C}$

additem cti_pt.frq cti_pt.frq interval $4 \quad 4 \quad \mathrm{~N} 2$

additem cti_pt.frq cti_pt.frq selval $44 \mathrm{~N} 1$

additem cti_pt.frq cti_pt.frq subbasin 44 I

/*Go Into Tables and Determine Range of Values tables

sel cti_pt.frg

\&sv selset $=[$ extract 1 [show number select] $]$

/*Get Value of Min. Record (Freq. Data Should Already be Sorted) \&Sv minval $=$ [SHOW RECORD 1 ITEM code]

/*Get Value of Max. Record

\&Sv maxval = [SHOW RECORD $\%$ selset\% ITEM code]

/*Determine Sample Interval

\&sv coreval $=[\mathrm{calc} \% \operatorname{maxval} \%$ - \%minval\%]

\&sv interval $=[$ calc $\%$ coreval\% $/$ opercentile $\%]$

calc interval = 을 inval\%

/*Loop Through and Flag Records For Dumping

\&Sv count $=1$

\&do \&until \%count\% gt \%percentile\%

sel cti pt.frg

/*Determine Selection Value

\&if $\frac{\circ}{c}$ count $\%=1$ \&then 


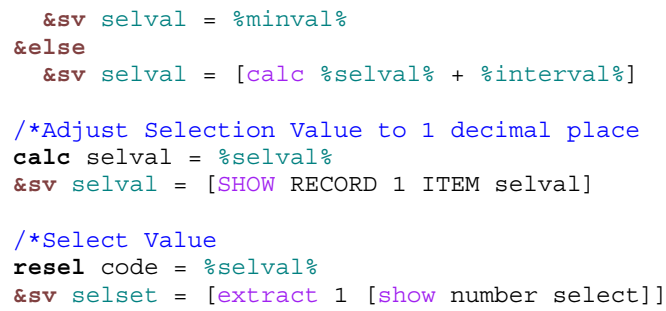

/*Unload Values For To set a Previous Value Record (should next value turn up 0's) \&if $\%$ selset\% $=1$ \&then \&do

\&if [exists cti pt.prv-file] \&then

\&sys rm cti_pt.prv

unload cti_pt.prv CASE\# FREQUENCY CODE EXTRACT INTERVAL SELVAL SUBBASIN send /*End unload of selected data record

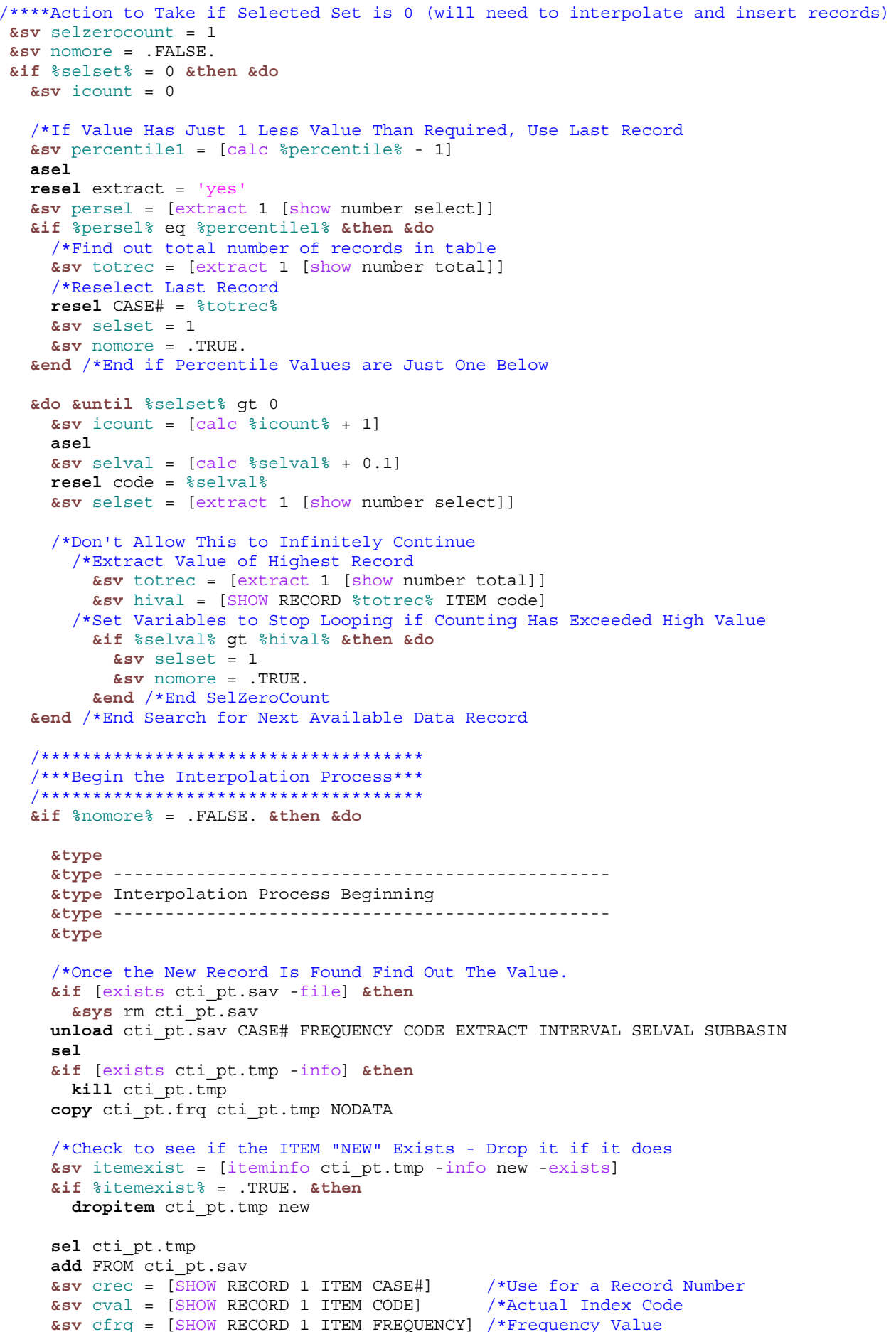




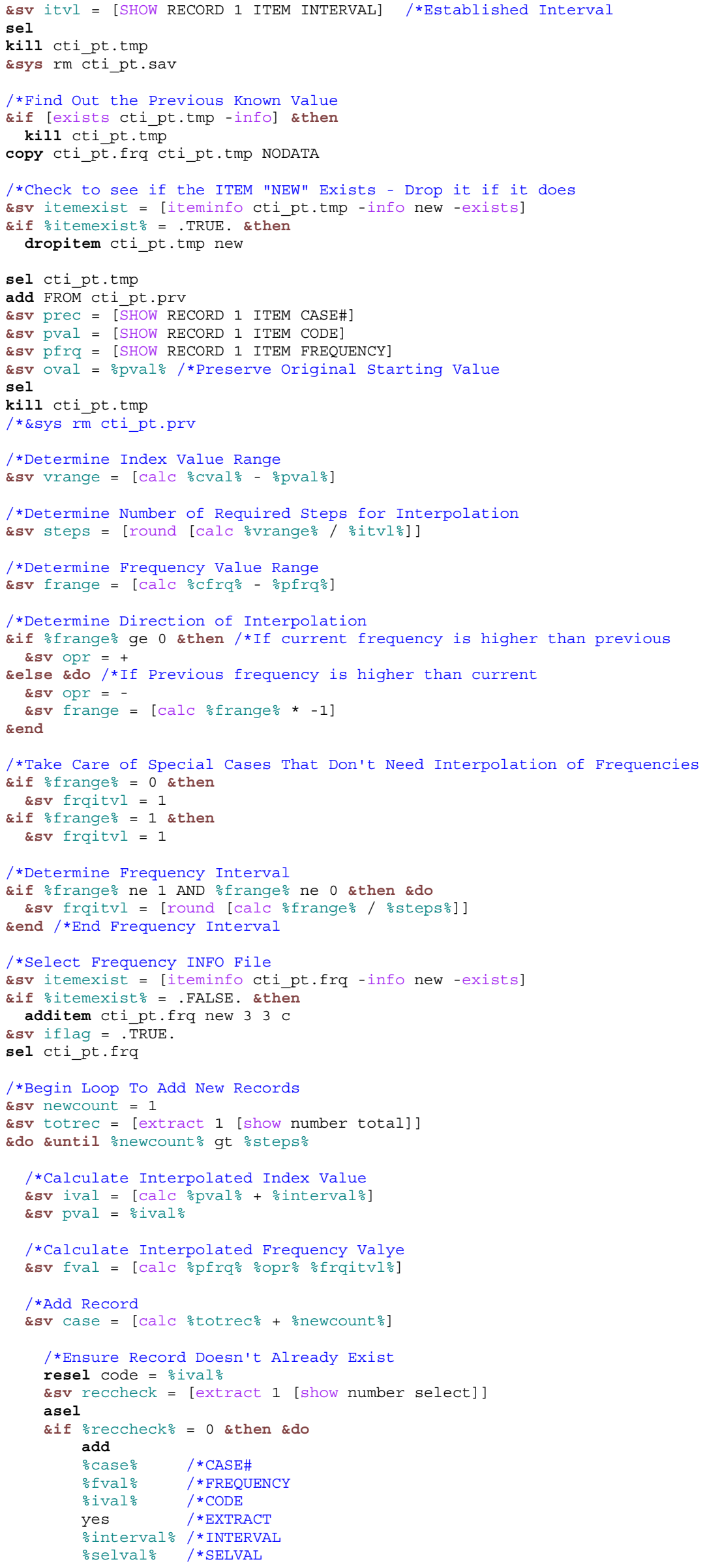




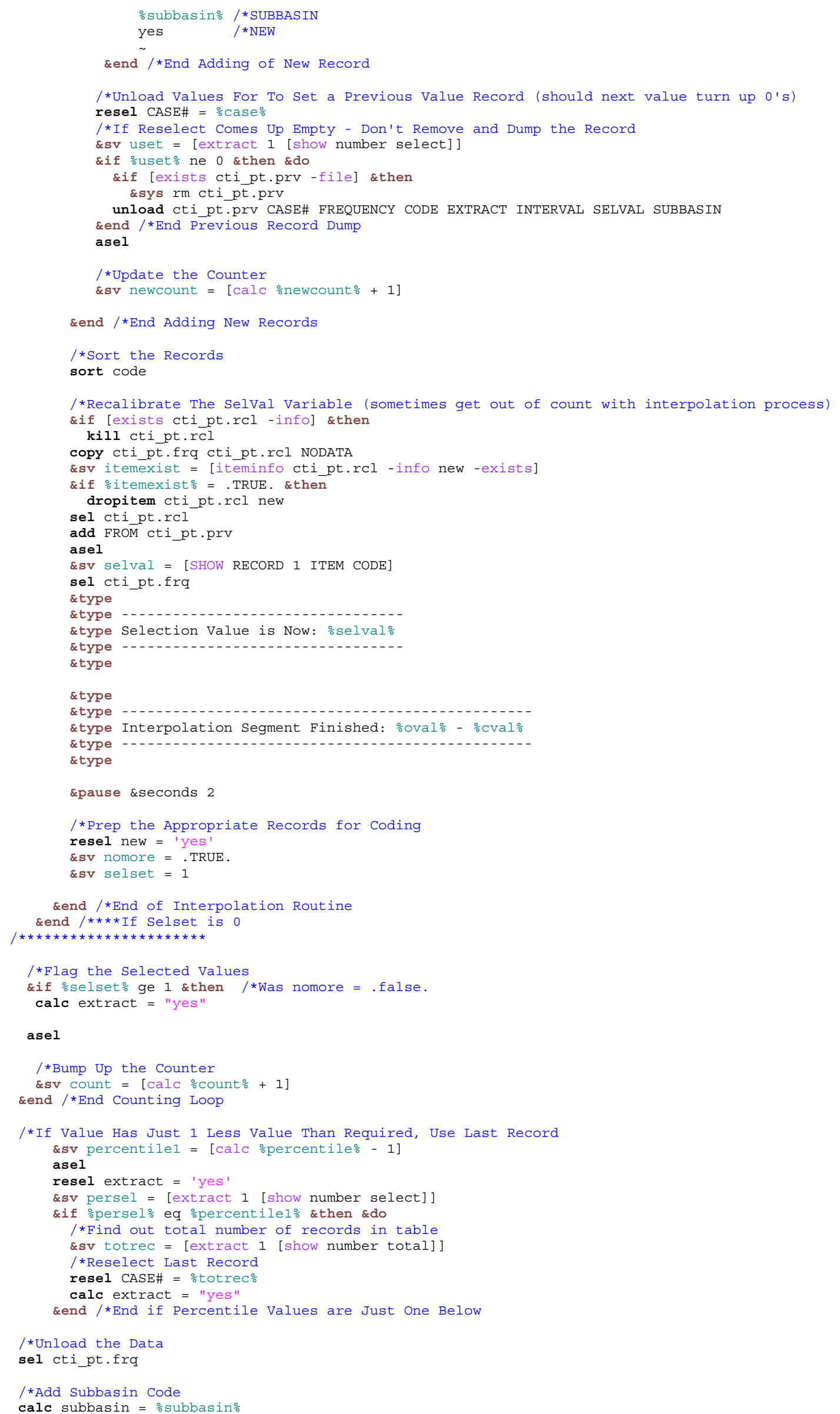


resel extract $=$ 'yes'

/*Write Note to Warning File if datasets have less or more than opercentile\% records /*Determine how many records are selected

\&sv numsel $=$ [extract 1 [show number select]]

\&if onumsel\% ne \%percentile\% \&then \&do

\&sys touch \%outdir\%:slash\%output\%slash\%warning.txt

\&sys echo Subbasin $\%$ subbasin\% contains \%numsel\% records. > \%outdir\%:slash\%output\%slash\%warning.txt

send

/*Dump Data to Text File

\&if [exists \%subbasin\%.cti -file] \&then

\&sys rm \%subbasin\%.cti

\&sys touch $\%$ subbasin\%.cti

\&sys echo "BASIN ID, CTI VALUE, FREQUENCY" >> \%ubbasin\%.cti

unload $\%$ subbasin\%.cti subbasin code frequency

quit /*Out of Tables

\&sys touch \%outdir\%:slash\%output $\%$ slash $\%$ subbasin $\%$.cti

\&sys cat \%subbasin .cti > \%utdir\%.slash\%output\%slash\%.subbasin\%.cti

\&work $\%$ scriptdir\%

\&return

/* Bailout Routine

sroutine bailout

\&type

\&type

stype AMT dump sb values.

\&type AML dump_sb_values.aml has encountered a fatal error. Stopping...

\&stop

\&return 


\section{[ - runsom.sh - ]}

$\# ! / \mathrm{bin} / \mathrm{sh}$

\#\#Run SOM Processor

\#\#Execute SOM

java -jar./sommer.jar --training-param-file batchl. dat

\#java -jar./sommer.jar --training-param-file batch2. dat

\#java -jar./sommer.jar --training-param-file batch3.dat

Hava -jar /sommer.jar --training-param-file batch4. dat

\#java -jar./sommer.jar --training-param-file batch5.dat

\#java -jar./sommer.jar--training-param-file batch6.dat

\#\#Run Post-Process

./postproc pkflow.rpt

exit 0 


\section{[ - batch1.dat - ]}

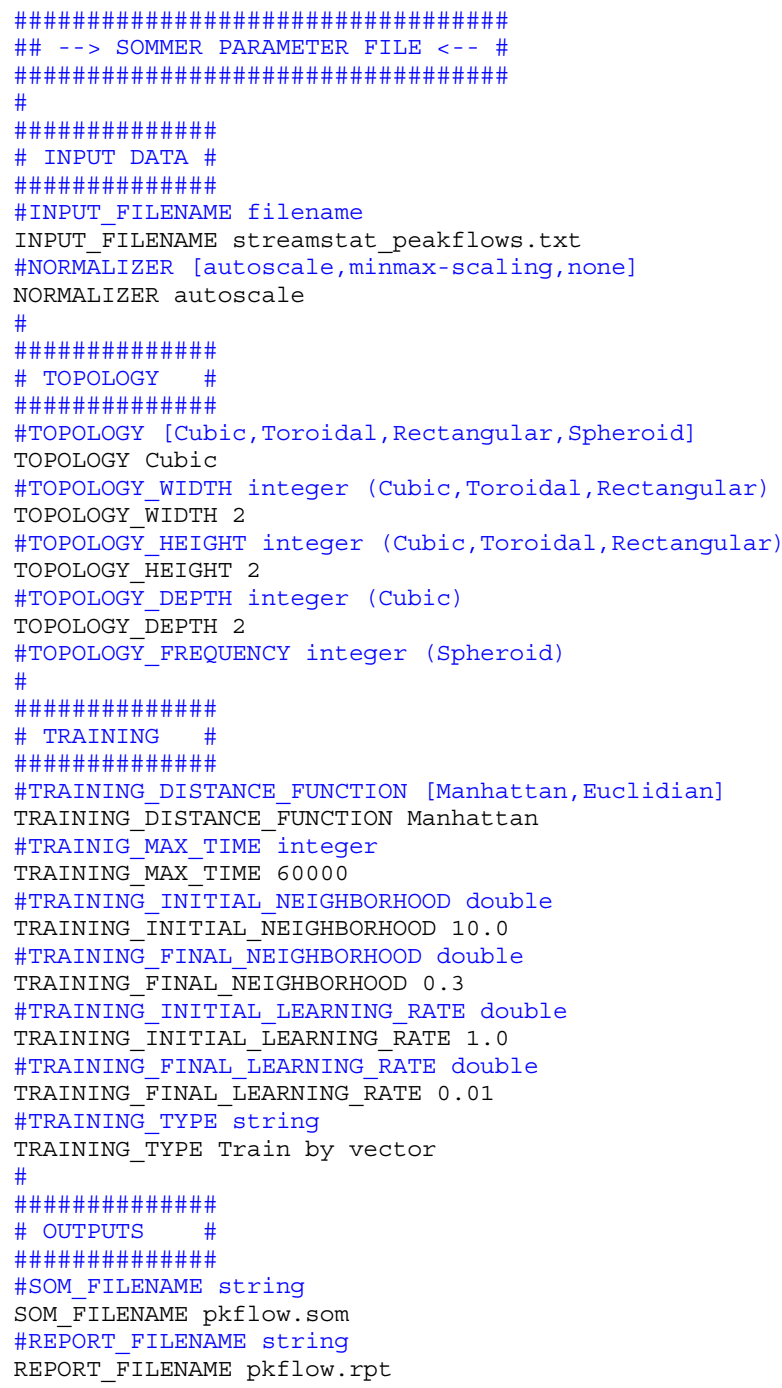




\section{[ - sompostproc.sh - ]}

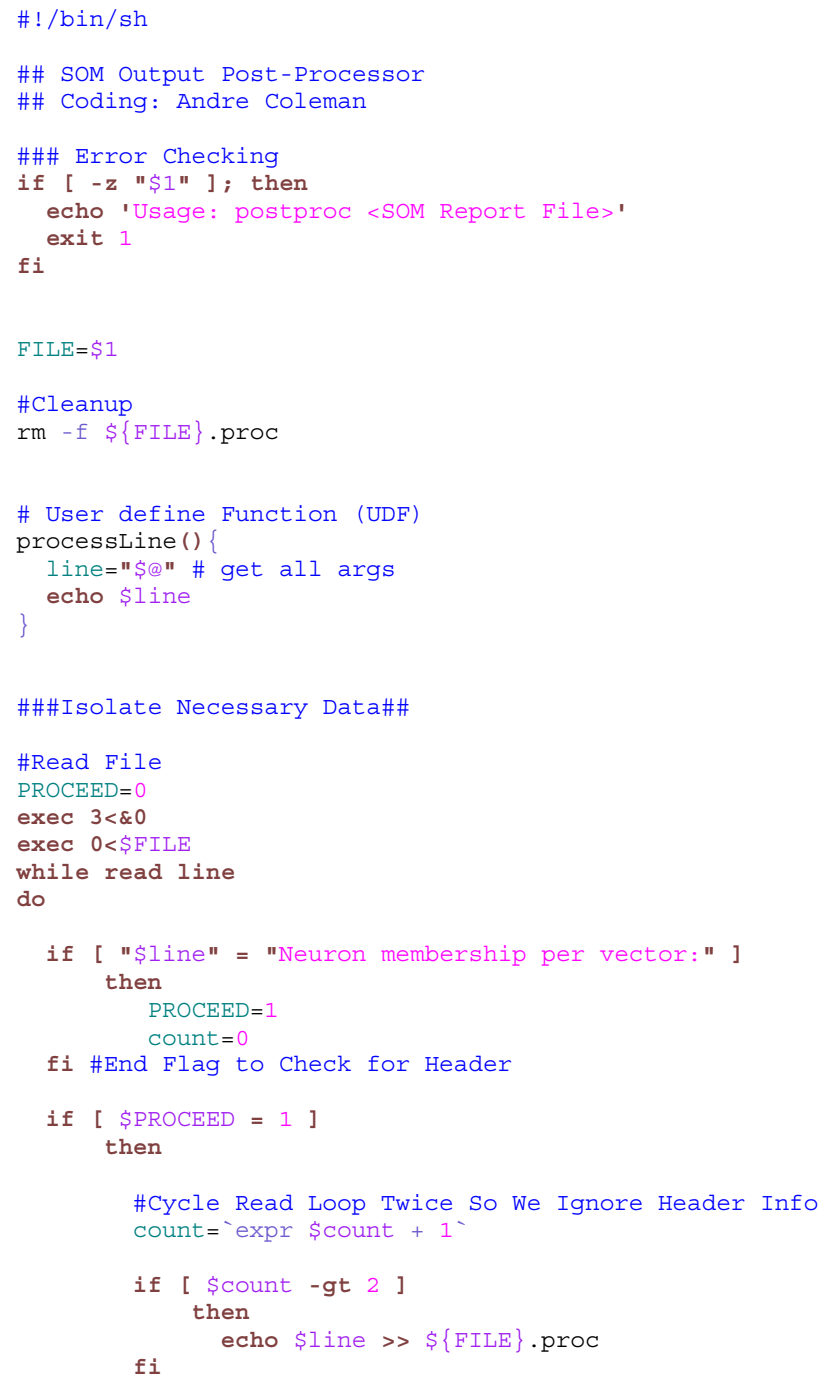

fi \#\#nd Write Cycle

done

exec $0<\& 3$

\#Remove middle column of data and write out GIS ready file

cat $\$\{$ FILE $\}$.proc | awk - F " " '\{print $\$ 1 ", " \$ 3\} '>\$\{F I L E\}$.gis

echo "Finished "

echo " "

echo "Post-Processing of SOM report file complete, please run JOINSOM.AML" echo " "

exit 0 


\section{[ - joinsom.aml - ]}

/** AML to join SOM class data back into the GIS by using the spatial container $/ * *$ unique id as the index (join) value.

/** Coding: Andre Coleman

\&args somrpt joincover jitem new_item

\&if [null onew_item\%] \&then \&do

\&type Usage: JOINSOM <SOM REPORT GIS FILE> <JOIN COVERAGE> <JOIN ITEM> <NEW ITEM>

\&type -SOM Report File needs to be two column comma-delimited

stype taking form of: ID, SOM CLS (1,x0y1z0). Look at \&type postproc.sh to process this for you.

\&type

stype -A file named 'asciizinfo.def' file needs to exist in

\&type current run directory. This defines the attribute space.

$$
\text { \&return }
$$

\&end

\&sv itemexist $=$ [iteminfo $\%$ joincover\% - poly $\%$ new_itemo -exists]

\&if $\%$ itemexist: $=$. TRUE. \&then

dropitem $\%$ joincover\%.pat $\%$ joincover\%. pat \%new_item\%

/*Convert SOM Report File to INFO File

asciizinfo \%somrpt\% asciizinfo.def $\frac{\circ}{\circ}$ oincover\%.dat ', '

/*Join INFO file to Coverage

joinitem $\%$ joincover\%.pat $\%$ joincover\%. dat $\%$ joincover\%.pat $\%$ jtem\%

$/ *$ Cleanup

killinfo \%joincover\%.dat

\&return 


\section{[ - ascii2info.def - ]}

$\begin{array}{llll}\text { BASINNUM } & 4 & 4 & \text { I } \\ \text { PKFLOW_CLS } & 6 & 6 & \text { C }\end{array}$

\section{[ - ascii2info.aml - ]}

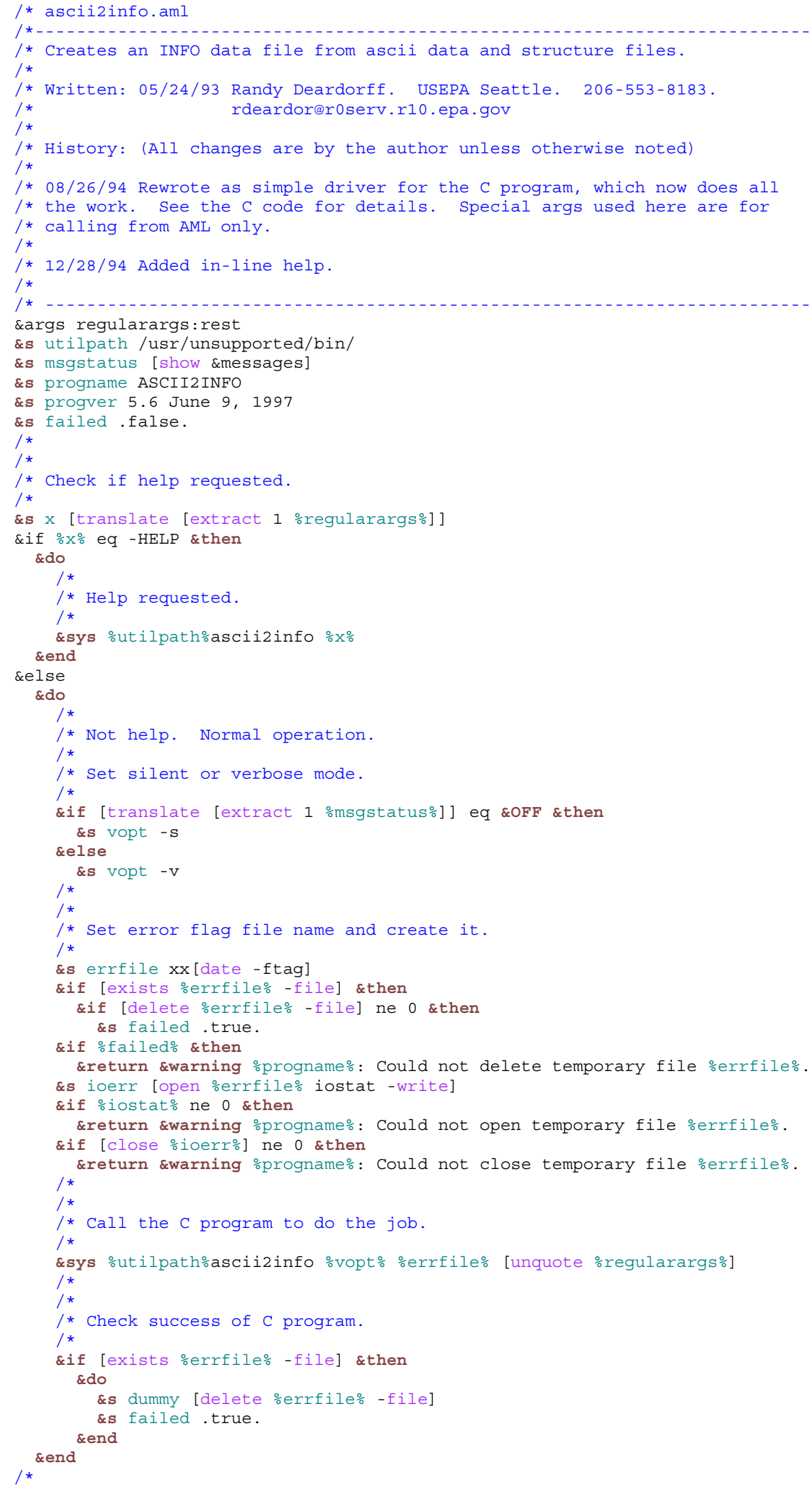


/* Return appropriate severity to calling program.

/*

\&if ㄷailed\% \&then

\&return \&warning

\&else

\&return 


\subsection{Appendix B}

Regression equations used for the estimation of monthly flow exceedence and flood frequency values in the North Fork of the Clearwater River watershed where discharge values $Q .80$ and $Q .20$ refers to the daily mean discharge (cubic feet per second) exceeded 80 and 20 percent of the time, respectively, during the course of the specified month, $A$ is drainage area in square miles, $B R^{*}$ is basin relief in thousands of feet, $p$ is mean annual precipitation in inches, $F *$ is forested area in percent, and $E^{*}$ is mean basin elevation in thousands of feet, (Hortness and Berenbrock, 2001).

\section{Monthly Flow Exceedence Equations}

October

$$
\begin{aligned}
& Q .80=6.35 \times 10^{-3} A^{0.916} B R * *^{0.656} p^{0.892} \\
& Q .20=1.86 \times 10^{-4} A^{0.822} B R * *^{1.09} p^{1.97}
\end{aligned}
$$

November

$$
\begin{aligned}
& Q .80=2.53 \times 10^{-3} A^{0.925} B R *^{0.552} p^{1.21} \\
& Q .20=4.23 \times 10^{-4} A^{0.945} B R * *^{0.416} p^{2.01}
\end{aligned}
$$

\section{December}

$$
\begin{aligned}
& Q .80=1.52 \times 10^{-7} A^{0.990} F * *^{1.43} p^{2.26} \\
& Q .20=2.34 \times 10^{-8} A^{0.983} F *{ }^{2.05} p^{2.36}
\end{aligned}
$$

\section{January}

$$
\begin{aligned}
& Q .80=7.62 \times 10^{-8} A^{0.997} F *{ }^{1.76} p^{2.05} \\
& Q .20=6.62 \times 10^{-9} A^{0.953} F *{ }^{2.94} p^{1.71}
\end{aligned}
$$

\section{February}

$$
\begin{aligned}
& Q .80=3.23 \times 10^{-8} A^{0.988} E *^{-0.292} F *^{2.11} p^{2.04} \\
& Q .20=1.73 \times 10^{-5} A^{0.957} E *^{-2.19} F * *^{1.56} p^{2.17}
\end{aligned}
$$

March

$$
\begin{aligned}
& Q .80=4.03 \times 10^{-7} A^{0.996} E *^{-1.00} F *^{2.15} p^{1.74} \\
& Q .20=2.51 \times 10^{-6} A^{0.981} E *^{-1.00} F * *^{2.22} p^{1.87}
\end{aligned}
$$


April

$$
\begin{aligned}
& Q .80=1.21 \times 10^{-8} A^{0.999} E *^{-0.783} F *^{2.45} p^{2.46} \\
& Q .20=1.26 \times 10^{-6} A^{0.978} E *^{-0.480} F *^{1.87} p^{2.10}
\end{aligned}
$$

May

$Q .80=1.07 \times 10^{-12} A^{1.03} E *{ }^{3.18} F *^{3.35} p^{2.36}$

$Q .20=1.38 \times 10^{-9} A^{0.984} E *{ }^{2.39} F *^{2.51} p^{2.08}$

June

$$
\begin{aligned}
& Q .80=2.87 \times 10^{-9} A^{0.800} E *^{2.39} B R *^{1.60} F *^{1.78} p^{1.95} \\
& Q .20=3.50 \times 10^{-10} A^{0.791} E *^{2.34} B R *^{1.74} F *^{2.56} p^{1.86}
\end{aligned}
$$

July

$$
\begin{aligned}
& Q .80=1.26 \times 10^{-8} A^{0.793} E *^{1.47} B R *^{1.44} F *^{1.88} p^{1.62} \\
& Q .20=1.61 \times 10^{-7} A^{0.755} E * *^{1.82} B R *^{1.73} F * *^{1.40} p^{1.54}
\end{aligned}
$$

August

$$
\begin{aligned}
& Q .80=1.03 \times 10^{-10} A^{0.963} E *^{2.58} F *^{2.92} p^{1.43} \\
& Q .20=1.07 \times 10^{-8} A^{0.947} E *{ }^{2.78} F * *^{2.10} p^{1.26}
\end{aligned}
$$

\section{September}

$$
\begin{aligned}
& Q .80=3.75 \times 10^{-11} A^{0.962} E *^{2.06} F *^{3.28} p^{1.43} \\
& Q .20=9.62 \times 10^{-8} A^{0.937} E *{ }^{2.10} F * *^{1.76} p^{1.30}
\end{aligned}
$$

Mean Annual

$Q a=5.14 \times 10^{-6} A^{0.986} F *^{9.42} p^{2.33}$

\section{Flood Frequency Equations}

\section{2-year Frequency}

$Q_{2}=0.742 * D A^{0.897} P^{0.935}$

\section{5-year Frequency}

$$
Q_{5}=1.50 * D A^{0.888}\left(\frac{E}{1000}\right)^{-0.330} P^{0.992}
$$

\section{0-year Frequency}

$$
Q_{10}=2.17 * D A^{0.884}\left(\frac{E}{1000}\right)^{-0.538} P^{1.04}
$$




\section{5-year Frequency}

$Q_{25}=3.24 * D A^{0.879}\left(\frac{E}{1000}\right)^{-0.788} P^{1.10}$

50-year Frequency

$Q_{50}=4.22 * D A^{0.876}\left(\frac{E}{1000}\right)^{-0.962} P^{1.14}$

100-year Frequency

$Q_{100}=5.39 * D A^{0.874}\left(\frac{E}{1000}\right)^{-1.13} P^{1.18}$

200-year Frequency

$Q_{200}=6.75 * D A^{0.872}\left(\frac{E}{1000}\right)^{-1.29} P^{1.21}$

500-year Frequency

$Q_{500}=8.90 * D A^{0.869}\left(\frac{E}{1000}\right)^{-1.49} P^{1.26}$ 